\title{
47th ESCP symposium on clinical pharmacy Personalised pharmacy care. 24-26 October 2018, Belfast, Northern Ireland
}

Published online: 29 November 2018

(C) Springer Nature Switzerland AG 2018

\section{Oral communication I: Community pharmacy}

CP-PC001: Effects of drug use reconsidered in the elderly using goal attainment scales during medication review: DREAMeR results of a randomized controlled trial

S. Verdoorn ${ }^{1,2, *}$, H.-F. Kwint ${ }^{1}$, J. Blom ${ }^{3}$, J. Gussekloo ${ }^{3,4}$, M. Bouvy ${ }^{1,2}$

${ }^{1}$ SIR Institute for Pharmacy Practice and Policy, Leiden, ${ }^{2}$ Division of Pharmacoepidemiology \& Clinical Pharmacology, Utrecht Institute for Pharmaceutical Sciences (UIPS), Utrecht, ${ }^{3}$ Department of Public Health and Primary care, ${ }^{4}$ Department of Gerontology and Geriatrics, Leiden University Medical Center (LUMC), Leiden, Netherlands

Background and objective: A clinical medication review (CMR) can reduce drug-related problems (DRPs). There is little evidence for effects on clinical outcomes. To improve quality of life a CMR could be more focused on health-related complaints and wishes of older persons. Therefore, the objective of this study is to investigate the effects of a CMR, focused on personal goals, on quality of life and health-related complaints in older patients with polypharmacy.

Setting and method: The DREAMeR study was a randomized controlled trial performed in 35 community pharmacies located throughout the Netherlands. 629 persons aged 70 years and over using seven or more chronic drugs were randomly assigned to usual care ( $\mathrm{n}=314$; control group) or to receive a CMR $(\mathrm{n}=315$; intervention group) and were measured after 3 and 6 months.

Main outcome measures: Health-related quality of life (HR-QoL) was measured with EQ-5D-5L (utility) and EQ-VAS. Complaints were defined as number of complaints independent of severity and number of complaints with impact on daily life (VAS-score $\geq 5$ and influence on daily life on 5-point Likert scale: moderate-severe). Also, the number of drugs in use were measured. Effects were analysed with linear mixed model analysis.

Results: Over 6 months, the utility measured with EQ-5D-5L remained equal between both groups $(\beta=0.0011 ; p=0.90)$ and the total number of complaints, irrespective of severity, did not change $(\beta=-0.14 ; p=0.12)$. The self-rated HR-QoL (EQ-VAS) increased over 6 months in the intervention group $(\beta=1.7 ; p<0.01)$ compared to control group and the number of complaints with impact on daily life decreased in the intervention group $(\beta=-0.17$; $p=0.029$ ). The number of drugs in use decreased over time in the intervention group $(\beta=-0.054$ per month; $p<0.05)$.
Conclusion: A clinical medication review focused on personal goals, improves the self-rated quality of life of older patients with polypharmacy and reduces the number of health-related complaints with impact on daily life, while the number of drugs in use decreases. Disclosure of Interest: None Declared.

CP-PC002: Outcomes of urinary tract infection management by pharmacists (RxOUTMAP): a study of pharmacist prescribing and care in patients with uncomplicated urinary tract infections in the community

\section{R. T. Tsuyuki ${ }^{1, *}$, N. Beahm ${ }^{2}$, D. Smyth ${ }^{3}$}

${ }^{1}$ Medicine, Faculty of Medicine and Dentistry, University of Alberta, ${ }^{2}$ EPICORE Centre, Faculty of Medicine and Dentistry, University of Alberta, Edmonton, ${ }^{3}$ Medicine, Horizon Health, Moncton, Canada

Background and objective: Pharmacists have the authorization to prescribe medications for the treatment of uncomplicated urinary tract infections (UTI) in some provinces. However, there is limited data on the outcomes of this care by pharmacists. Our objective was to evaluate the effectiveness, safety, and patient satisfaction with pharmacist prescribing and care in patients with uncomplicated UTI. Setting and method: We conducted a prospective registry trial in 39 community pharmacies in the Canadian province of New Brunswick. Adult patients were enrolled if they presented to the pharmacy with either symptoms of UTI with no current antibacterial treatment (Pharmacist-Initial Arm) or if they presented with a prescription for an antibacterial to treat UTI from another health care provider (Physician-Initial Arm). Pharmacists assessed patients and if they had complicating factors or red flags for systemic illness or pyelonephritis, they were excluded from the study. Pharmacists either prescribed antibacterial therapy, modified antibacterial therapy, provided education only, or referred to physician, as appropriate.

Main outcome measures: The primary outcome was clinical cure at 2 weeks and the secondary outcomes included adverse events and patient satisfaction.

Results: A total of 748 patients were enrolled (87\% in the Pharmacist-Initial Arm), average age was 40.8 (SD 15.9). Clinical cure was achieved in $89.1 \%$ of patients. Of those that did not have sustained symptom resolution, most $(6.1 \%)$ had symptom recurrence after completion of therapy. Adverse events were reported by $6.8 \%$ of patients and $88.2 \%$ of those continued their medication. Most adverse 
events were gastrointestinal-related and transient. The patient satisfaction survey reflected very high levels of satisfaction for the care they received, as well as for trust and accessibility of the pharmacist. Conclusion: Pharmacist management of uncomplicated UTIs is effective, safe, and patient satisfaction is very high.

Disclosure of Interest: None Declared.

CP-PC003: Development of a theory-based intervention to improve medicines management for people with dementia in primary care

H. E. Barry ${ }^{1}{ }^{*}$, L. Bedford ${ }^{1}$, M. McGrattan ${ }^{1}$, C. Ryan $^{2}$, A. P. Passmore ${ }^{3,4}$, A. L. Robinson ${ }^{5}$, G. J. Molloy ${ }^{6}$, C. M. Darcy ${ }^{7}$, H. Buchanan ${ }^{8}$, C. M. Hughes ${ }^{1}$

${ }^{1}$ School of Pharmacy, Queen's University Belfast, Belfast, United Kingdom, ${ }^{2}$ School of Pharmacy and Pharmaceutical Sciences, Trinity College Dublin, Dublin, Ireland, ${ }^{3}$ Belfast Health and Social Care

Trust, ${ }^{4}$ Centre for Public Health, Queen's University Belfast, Belfast, ${ }^{5}$ Institute for Ageing and Health, Newcastle University, Newcastle, United Kingdom, ${ }^{6}$ School of Psychology, National University of Ireland, Galway, Ireland, ${ }^{7}$ Western Health and Social Care Trust, Londonderry, ${ }^{8}$ Unspecified, Belfast, United Kingdom

Background and objective: People with dementia (PWD) have additional medication needs compared with other older people. Research and healthcare policy emphasise the importance of optimising medicines management in PWD, yet limited primary carebased research exists. ${ }^{[1]}$ This study aimed to develop a theory-based intervention to improve medicines management for PWD in primary care.

Design: Medical Research Council guidance advocates systematic identification of theory and evidence to underpin intervention development. ${ }^{[2]}$ The Theoretical Domains Framework (TDF) ${ }^{[3]}$ was used to explore general practitioners' (GPs') and community pharmacists' perceptions of facilitators and barriers to successful medicines management (i.e. prescribing, dispensing, conducting medication review, monitoring adherence-target behaviours) for PWD, using a qualitative approach. Key theoretical domains were identified and mapped to behavioural change techniques (BCTs-active components of an intervention) using an established taxonomy. ${ }^{[4]}$ Following discussion within the research team, draft interventions were developed to operationalise selected BCTs. These were presented to GPs and pharmacists during task groups; participants were asked to assess feasibility of implementing proposed intervention content using APEASE (Affordability, Practicability, Effectiveness/cost-effectiveness, Acceptability, Side-effects/safety, Equity) criteria. Ethical approval was granted for this study (Ref: 15/EE/0103).

Results: Two draft interventions comprising selected BCTs ('Modelling or demonstration of behaviour', 'Salience of consequences', 'Information about health consequences', 'Information about social and environmental consequences', 'Action planning', 'Social support or encouragement', 'Self-monitoring of behaviour') were developed, each targeting GPs or community pharmacists. Following task groups, and research team discussions, the community pharmacist intervention was selected for feasibility testing. The intervention will target community pharmacists to conduct a medication review and monitor adherence in a PWD, delivered as an online video demonstrating key behaviours. The video will include feedback emphasising positive outcomes of performing the behaviours. Action planning and a 'quick reference guide' will be used as complementary intervention components to facilitate conducting medication review and monitoring adherence in PWD.

Conclusion: A community pharmacy-based intervention has been developed targeting medicines management for PWD using a theorybased approach. Future work will involve feasibility testing of the intervention in two community pharmacies to determine if additional refinements are required before progressing to a pilot study.

Disclosure of Interest: None Declared.

CP-PC004: Clinical impact of pharmacist interventions relative to renal function in $\mathbf{7 0}$ French community pharmacies by nephrologists and general practitioners: a multicentre prospective study

F. Slimano 1,2,*, A. Lestrille ${ }^{3}$, L. Aubert ${ }^{1,2}$, P. Kreit ${ }^{4}$, E. Herlem ${ }^{5}$,

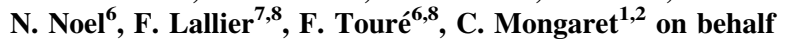
of the MIRPhO investigators

${ }^{1}$ Faculty of Pharmacy, Reims University, ${ }^{2}$ Department of Pharmacy, CHU Reims, ${ }^{3}$ Pharmacie d'officine Croix du Sud, Reims, ${ }^{4}$ URPS Pharmaciens Grand Est, Nancy, ${ }^{5}$ General practitioner office, ${ }^{6}$ Nephrology, CHU Reims, Reims, ${ }^{7}$ General practitionner office, Ville-en-Tardenois, ${ }^{8}$ Faculty of Medicine, Reims University, Reims, France

Background and objective: The prescription validation activity in community pharmacy should include the glomerular filtration rate (GFR) especially in the elderly but this data is not available in France for community pharmacists (CPs). We recently conducted a 6-month multicentre prospective study (for patient $\geq 65$ years) to assess the impact GFR availability on the prescription validation in terms of drug-related problems (DRP), pharmacist interventions (PI) and acceptance rate. All records were independently double-assessed by an Expert committee (four hospital and community pharmacists with training in clinical pharmacy).

Setting and method: In the prospective study there were $n=445$ patients with GFR $<60 \mathrm{~mL} / \mathrm{min}$ ( $21.6 \%$ of all inclusions). CPs detected 99 DRPs while the expert committee (Experts) detected 184 DRPs. Two nephrologists and two general practitioners (GP) rated the clinical impact for all PI using the 4-level Chedru tool (derived from Hatoum). Additionally, clinicians could rate "-1" when PI seemed to be harmful for patient's health (modified Chedru-mChedru). PIs rated by all clinicians were analysed using $R^{\circledR}$ (v.3.2.2). The significance level of alpha was 0.05 .

Main outcome measures: Primary endpoint was to assess the clinical impact of PI performed in the 70 community pharmacies (when PI was rated $\geq 1$ by clinicians). Secondary endpoints were to assess clinical impact between CPs and Experts, and accordance between clinicians.

Results: $\mathrm{N}=74$ PIs made by CPs and $\mathrm{n}=146$ PIs by Experts were rated by all clinicians. The clinical impact $(\geq 1)$ varied from 51 to $59 \%$ for PIs by CPs and from 59 to $82 \%$ for PIs made by Experts depending on clinicians. PIs made by Experts were more relevant than CPs $(p<0.05)$. With mChedru nephrologists rated "- 1 " for $41 \%$ of PIs made by CPs and $8 \%$ of PIs by Experts. PIs made by Experts were also more clinically relevant $(p<0.05)$. The main reason for rate "1 " by clinicians was PIs performed in order to reduce dosage of angiotensin-converting-enzyme inhibitor (ACEi). The accordance rate between clinician was significantly correlated $(p<0.05)$ with low or moderate evidence (0.21-0.60).

Conclusion: French CPs have proven they can perform PIs using GFR and the related clinical impact seemed promising. The lack of training with GFR can probably explain why PIs by CPs were less relevant than PIs by Experts. For example, most PIs regarding ACEi were systematically classified as harmful by clinicians. Our results encourage the systematic availability of GFR for CP. This will be prepared for by appropriate training during and after Pharmacy education in order to improve the use of GFR by CPs and the clinical impact of their PIs.

Disclosure of Interest: None Declared. 


\section{CP-PC005: Identifying methods to prioritise patients} for deprescribing using a systematic review of deprescribing algorithms in elderly patients

\author{
M. Donovan ${ }^{1, *}$, M. O'Connell ${ }^{1}$, S. Byrne ${ }^{1}$ \\ ${ }^{1}$ School of Pharmacy, University College Cork, Cork, Ireland
}

Background and objective: Deprescribing is the process of either dose reduction or discontinuation of a medication that may be causing more harm than good for the patient. Deprescribing algorithms provide a stepwise approach to the process of deprescribing. This systematic review examined the availability of deprescribing algorithms for the elderly, the processes used to select patients for deprescribing, as well as the outcomes of deprescribing for this patient cohort. The information gathered in this review provides the basis for developing a stratification tool, which could be utilised by clinical pharmacists to identify patients who would benefit most from deprescribing.

Design: Based on PRISMA guidelines, a systematic electronic literature search was performed using four databases (PubMed, CINAHL, EMBASE and Web of Science) from inception up until November 2017. Studies were selected using predefined inclusion and exclusion criteria. Titles and abstracts were screened initially before full review of relevant papers. Risk of bias assessments were conducted on included studies.

Results: Eighty-five studies were screened and fourteen studies were included in the systematic literature review. Included studies were assessed to have a low to moderate risk of bias. Eleven deprescribing algorithms were reviewed; each algorithm was based on different criteria e.g. a number were based on the deprescribing of a large number of potentially inappropriate medications, while others were limited to a singular drug class. A number of different criteria were used to select patients for deprescribing; some studies included all patients within a certain age group, two studies included frail older adults only, some studies only included patients on polypharmacy, while the targeted deprescribing algorithm studies only required the patient to be taking one drug from a specific drug class. This information could be amalgamated to produce a stratification tool for identifying patients who may be suitable for deprescribing. The use of deprescribing algorithms in the elderly have been reported to benefit patient clinical outcomes in a number of ways: reduced prescribing of potentially inappropriate medications, reduced mortality, improved quality of life and reduced hospital admissions.

Conclusion: Deprescribing algorithms have been shown to be beneficial in the care of the elderly, primarily due to the reduction in the number of medications being taken. Deprescribing is a complex process, in which pharmacists could play a greater role. The identification of priority patients (who would benefit from deprescribing) by pharmacists in primary and secondary care would aid in streamlining the deprescribing process.

Disclosure of Interest: None Declared.

\section{CP-PC006: COM-MA: improving patient-centered} communication of pharmacy technicians in the outpatient pharmacy with an online feedback intervention

M. Vervloet ${ }^{1, *}$, E. Koster ${ }^{2}$, A. Lamboo ${ }^{3}$, A. Linn ${ }^{4}$, J. Noordman ${ }^{1}$, D. Philbert ${ }^{2}$, R. Linde ${ }^{5}$, B. van den Bemt ${ }^{6,7}$, L. van Dijk ${ }^{1}$

${ }^{1}$ NIVEL, ${ }^{2}$ Utrecht Pharmacy Practice Network for Education and Research, Utrecht University, Utrecht, ${ }^{3}$ Centre for Patient and Medication, Loenen, ${ }^{4}$ ASCoR, Amsterdam School for Communication Research, Amsterdam University, Amsterdam, ${ }^{5}$ Zorggroep Almere, outpatient pharmacy "de Brug", Almere,

${ }^{6}$ Department of Pharmacy, Radboud University Medical Center,
${ }^{7}$ Department of Pharmacy, Sint Maartenskliniek, Nijmegen, Netherlands

Background and objective: Pharmacy technicians are in an excellent position to detect and discuss medication-related problems at the counter. However, opportunities to discuss medication use with patients are often left unused. The objective of this study was to develop and test an online feedback intervention based on videorecordings, self-reflection and personal feedback (COM-MA) to improve pharmacy technicians' patient-centered communication skills.

Setting and method: COM-MA was tested using a pre-posttest design in three outpatient pharmacies in the Netherlands. COM-MA used video-recordings of patient-pharmacy technician conversations at the counter. These were made available for pharmacy technicians through the COM-MA webportal. After viewing their conversations, technicians completed a self-reflection task online. Using an observation protocol, all conversations were scored on seven core communication domains, for example picking up and addressing cues on concerns about the medication. Based on these scores and the selfreflection online personal feedback was given. Finally, pharmacy technicians were encouraged to reflect on the feedback and ask questions.

Main outcome measures: Improvement in the core communication domains.

Results: In total, 231 (141 before training and 90 after) conversations of 32 pharmacy technicians were video-recorded. They each received personal feedback on two conversations, consisting of their communication strengths ('tops') and suggestions for improvement ('tips'). Most frequently given tops were: (1) being friendly and calm, (2) clearly explaining use instructions in case of first prescriptions, (3) giving patients room for posing questions, (4) being patient-centered in starting and ending the conversation. Most frequently given tips were: (1) listen more attentively, (2) better tailor information to patients' needs, (3) better detect (implicit) concerns about the medication, (4) further exploring possible uncertainties of patients. After being trained, pharmacy technicians improved their information tailoring, their inquiry for patients' experiences with the medication, and their skills to enhance patient engagement in the conversation. Communication skills regarding identifying and addressing possible concerns did not improve. COM-MA was well appreciated by pharmacy technicians.

Conclusion: COM-MA provides pharmacy technicians with more insight in their patient-centred communication at the counter. Communication aspects that need more in-depth training, for example regarding addressing concerns, can be identified with COM-MA.

Disclosure of Interest: None Declared.

CP-CE001: General public awareness and views of community pharmacy services in Scotland: the 'First Port of Call' study

K. Maclure ${ }^{1, *}$, G. Craig' ${ }^{2}$ A. MacLure' ${ }^{1}$ A. Boyter ${ }^{3}$, A. Power ${ }^{2}$, A. Osprey ${ }^{4}$, A. McGregor ${ }^{5}$, D. Stewart ${ }^{1}$

${ }^{1}$ School of Pharmacy and Life Sciences, Robert Gordon University, Aberdeen, ${ }^{2}$ NES Pharmacy, NHS Education for Scotland,

${ }^{3}$ Strathclyde Institute of Pharmacy and Biomedical Sciences, University of Strathclyde, Glasgow, ${ }^{4}$ Community Pharmacy Scotland, ${ }^{5}$ Royal Pharmaceutical Society, Edinburgh, United Kingdom

Background and objective: The recently published 'Achieving Excellence in Pharmaceutical Care: a Strategy for Scotland' urges community pharmacy $(\mathrm{CP})$ to make itself the 'first port of call' for healthcare advice. The aim of this study was to explore the Scottish general public's awareness of $\mathrm{CP}$ services and their openness to consider CP their 'first port of call' for health care advice. 
Setting and method: A Scotland-wide survey was administered up to 20 times in each of 117 CPs by pre-registration pharmacy graduates. It included Likert scales of attitudinal statements with items developed from existing literature. Ethical approval had been gained.

Main outcome measures: General public awareness of community pharmacy services in Scotland.

Results: To date, the 2260 surveys have been completed from 117 CPs giving a response rate of $96.5 \%$. Varying ages and standards of health are represented. Respondents were aware CPs 'are contracted to the NHS' (87.4\%) and that 'pharmacists with an additional qualification can diagnose and prescribe' (53.3\%). $87.6 \%$ would likely/ very likely view CP as their 'First port of Call' for common illnesses. However, $71.4 \%$ were unlikely/very unlikely to approach CP with 'more serious symptoms'. Intentions for 'monitor or review of regular medicines' were less clear (unlikely/very unlikely (38.2\%) against likely/very likely (35.1\%)). 44\% lacked an awareness of CP services in general. $75.3 \%$ indicated the pharmacist 'should have access to read and update relevant parts of my electronic health record' and pharmacist access to electronic records would make $67.8 \%$ more likely to view $\mathrm{CP}$ as a 'first port of call' for health issues.

Conclusion: This Scotland-wide survey indicated the general public's positivity and openness to access community pharmacy services, however, being recognised as a 'First Port of Call' may require awareness raising.

Disclosure of Interest: None Declared.

PT001: A survey of prescribers in the Scottish Highlands on their perspectives of prescribing direct-acting oral anticoagulants

D. Generalova $^{1, *}$, S. Cunningham ${ }^{1}$, S. Leslie ${ }^{2}$, G. Rushworth ${ }^{3}$, L. McIver ${ }^{4}$, D. Stewart ${ }^{1}$

${ }^{1}$ School of Pharmacy and Life Sciences, Robert Gordon University, Aberdeen, ${ }^{2}$ Department of Medicine, Raigmore Hospital, ${ }^{3} 3$ Highland Pharmacy Education and Research Centre, Centre for Health Science, Inverness, ${ }^{4}$ Pharmacy, Healthcare Improvement Scotland, Glasgow, United Kingdom

Background and objective: The introduction of dabigatran in 2008, followed by other direct-acting oral anticoagulants (DOACs), rivaroxaban, apixaban and edoxaban, has led to significant changes in the management of non-valvular atrial fibrillation (AF). The objective of this research was to determine prescribers' views and experiences relating to prescribing DOACs for the management of non-valvular AF.

Setting and method: The research included all prescribers practising within the Scottish Highlands. A questionnaire was developed based on the published literature, with items on influences of prescribing derived from the Theoretical Domains Framework of behavioural change. Following pre-testing and piloting, an email containing a link to the questionnaire was sent to all registered prescribers. Data were analysed descriptive statistics, principal component analysis, inferential testing and content analysis of responses to open questions. The study was approved by a university ethics committee and the regional Research and Development committee.

Main outcome measures: Views and experiences of prescribing DOACs.

Results: Of the 154 responses received, 16 were excluded as having never prescribed DOACs. One hundred and twenty responses $(77.9 \%)$ were from doctors, $18(11.7 \%)$ from nurse prescribers and $10(6.4 \%)$ from pharmacist prescribers ( 6 missing). PCA gave four components: the role of professionals and their knowledge and skills; influences on prescribing; consequences of prescribing; and monitoring for safety and effectiveness. While the scores for three components were generally high, the lowest scores (indicating less favourable views) related to consequences of prescribing DOACs rather than warfarin in terms of effectiveness, cost-effectiveness and safety. Those with less years of prescribing experience scored statistically significantly higher than others $(p<0.05)$. Content analysis identified experiences of advantages (e.g. absence of INR monitoring) and disadvantages (e.g. adverse effects of bleeding).

Conclusion: The results of this study suggest that that those responding had generally positive views and experiences of prescribing DOACs for non-valvular AF. There may need to be increase awareness of the evidence base of efficacy, effectiveness and safety. Furthermore, all prescribers should be encouraged to report adverse effects to national and international pharmacovigilance organisations. Disclosure of Interest: None Declared.

\section{Oral communication II: Hospital pharmacy}

HP-PC001: Screening model detecting patients who may benefit from a pharmacist-led medication review

\section{T. R. Nielsen ${ }^{1, *}$, L. Midjord Weisbjerg ${ }^{2}$}

${ }^{1}$ Clinical pharmacy, Region Zealand Hospital Pharmacy, Roskilde, ${ }^{2}$ Clinical pharmacy, Region Zealand Hospital Pharmacy, Næstved, Denmark

Background and objective: The Danish Health Authority national action plan describes a need for ensuring that relevant elderly polypharmacy patients receive medication reviews during hospital admission to reduce the risk of adverse events. Potentially Inappropriate Medications (PIMs) are one of the most frequent causes of adverse events in the elderly patients.

The objective was to develop a screening model to identify the patients who may benefit from a pharmacist-led medication review during admission.

Design: PIMs described in national and international literature was combined to a full list of 32 PIMs. The PIMs were then independently scored (1-3) by 14 experienced clinical pharmacists for the clinical impact if deprescribed, the likelihood of effecting deprescription during admission and for the ease of screening in the electronic patient record. The PIMs with the best combined scores were selected for the screening model.

The model was tested for sensitivity and specificity on a cohort of 72 elderly polypharmacy patients previously reviewed by clinical pharmacists of whom about half got a pharmacist intervention.

Sensitivity was calculated as (number of patients in the cohort with a pharmacist intervention who fit the screening model)/(number of patients with a pharmacist intervention).

Specificity was calculated as number of patients in the cohort without a pharmacist intervention who did not fit the screening model)/(number of patients without a pharmacist intervention).

Results: The screening model consists of 10 medication focus points. When applied to a cohort of elderly medical patients the model showed a sensitivity of $80 \%$ and a specificity of $78 \%$ in identifying the patients who received a pharmacist intervention. The screening model will be applied by pharmacy technicians screening all hospital patients and referring relevant patients to a pharmacist-led medication review.

Conclusion: The screening model identifies relevant elderly patients who may benefit from a pharmacist-led medication review during admission with the aim of reducing Potentially Inappropriate Medications.

Whether the elderly patients have a reduced number of medications and PIMs when discharged will be investigated in future studies.

Disclosure of Interest: T. Nielsen Grant/Research support-The study has been funded by The Danish Health Authority special pool of funds., L. Midjord Weisbjerg: None Declared. 
HP-PC002: An evaluation of a pharmacist led discharge service in an acute teaching hospital in Ireland

\author{
F. $\operatorname{Ryan}^{1}$, S. McCool ${ }^{1}$, S. Byrne ${ }^{2, *}$ \\ ${ }^{1}$ Pharmacy Department, St. Luke's Hospital, Kilkenny, ${ }^{2}$ School of \\ Pharmacy, University College Cork, Cork, Ireland
}

Background and objective: Medication errors can occur at any transition of patient care. However, evidence suggests that medication errors are more common on discharge. Medication reconciliation at transitions in a patient's care have been found to reduce the risk to patient safety and improve communication between care settings. The aim of this study was to determine if the discharge prescription for patients receiving the pharmacist led discharge service had a greater compliance with the Health Information and Quality Authority (HIQA) National Standard for Patient Discharge Summary Information than patients receiving standard pharmacy service and no pharmacy service.

Setting and method: The study was conducted in a University Teaching Hospital in southern Ireland. Discharge prescriptions were audited against the HIQA National Standard for Patient Discharge Summary Information. There were three arms to the study (1) the intervention group consisting of patients $(n=94)$ that received a pharmacist medication reconciliation on admission and discharge, along with preparation of the discharge prescription and communication of the discharge prescription to the GP and community pharmacy, (2) a control group consisting of patients $(n=100)$ which had a pharmacist medication reconciliation on admission only, and (3) a control group consisting of patients $(n=100)$ that received no pharmacy service. Satisfaction surveys were circulated to all stakeholders.

Main outcome measures: The main outcome measure was compliance with the Health Information and Quality Authority (HIQA) National Standard for Patient Discharge Summary Information.

Results: The found that pharmacist involvement in the preparation of a patient's discharge prescription improved the compliance with our National Standard for Patient Discharge Summary Information. Pharmacist prepared discharge prescriptions had fewer discrepancies than discharge prescriptions prepared by hospital doctors (99\% vs. $85 \%)$. GPs, Community Pharmacists also reported an improvement in the quality of the discharge prescription as determined by a satisfaction survey. The key benefits of the service identified by stakeholders were as follows (1) time saving, (2) increased patient safety, (3) fewer queries and (4) clearer prescriptions with more information.

Conclusion: This study has demonstrated that pharmacist involvement in the preparation of a patient's discharge prescription enhances compliance with the HIQA National Standard for Patient Discharge Summary Information.

Disclosure of Interest: None Declared.

\section{HP-PC003: Medication reviews in hospitals: an overview of published guidelines}

\section{H. Studer ${ }^{1, *}$, N. Felder ${ }^{1}$, K. E. Hersberger ${ }^{1}$, M. L. Lampert ${ }^{1,2}$}

${ }^{1}$ Pharmaceutical Care Research Group, University of Basel, Basel, ${ }^{2}$ Institute of Hospital Pharmacy, Solothurner Spitäler AG, Solothurn, Switzerland

Background and objective: Complex pharmaceutical interventions have been shown to reduce hospital readmission. In-hospital medication reviews conducted by a pharmacist were a key element of these complex interventions. The Pharmaceutical Care Network Europe (PCNE) proposed a definition and classification of different types of medication reviews. However, it often remains unclear how the medication reviews are performed and what type according to PCNE was applied.
The objective of this project was therefore to generate an overview of guidelines for in-hospital medication reviews.

Setting and method: We searched websites of pharmaceutical associations and societies for guidelines on medication reviews with focus on reviews conducted in the hospital. We also conducted an open internet search using a combination of the following search terms: medication review, medication/medicine use review, guideline, hospital, public pharmacy.

Guidelines for medication reviews in English and German were compiled in an overview.

Main outcome measures: Number and characteristics of guidelines for medication reviews conducted in hospitals.

Results: We identified 3 international and 12 national guidelines for medication reviews from associations and societies. Preliminary analysis showed that most guidelines recommend obtaining a medication history for the medication review; only two specified that it should be the best possible medication history. Medication reconciliation in combination with the medication review is recommended by seven guidelines.

Conclusion: As expected, most guidelines exist on a national level and only few on an international level. The structured overview may help identify core elements in order to establish a best practice standard for medication reviews in the hospital.

Disclosure of Interest: None Declared.

\section{HP-PC004: Patients' attitudes towards medication in mental} health care J. K. Vederhus ${ }^{1}$, Y. L. Hauge ${ }^{2}$, A. C. Irgens
, H. Hegevik
,

${ }^{1}$ Addiction Unit, Sørlandet Hospital, ${ }^{2}$ Sykehusapotekene, ${ }^{3}$ Sørlandet Hospital, Kristiansand, Norway, ${ }^{4}$ user representative, Sørlandet Hospital, Kristiansand, ${ }^{5}$ Department of Psychology, The Faculty of Social Sciences, ${ }^{6}$ Faculty of medicine, University of Oslo, Oslo, Norway

Background and objective: Patients' attitudes towards medication play an important role when medication is used for treatment and follow-up in mental health care services. We sought knowledge about patients' attitudes, which are thought to be influenced by multiple factors. Aim was to explore the patients' attitude towards medication in a mental health care setting, and to identify patient related factors associated with this.

Setting and method: A cross sectional study was performed where patients in contact with mental health care services at Sørlandet Hospital were included. All participating patients gave written consent. Demographic and clinical questions, a self-report questionnaire: Beliefs About Medicines Questionnaire BMQ, and self-designed questions regarding treatment satisfaction were asked for. For analyses SPSS analysis tool was used.

Main outcome measures: The patient reported attitudes about the two constructs positive and negative understanding of medication made concrete through the terms needs and concerns regarding medication, measured by the Beliefs About Medicines Questionnaire BMQ.

Results: The study included 992 patients. The patients reported a stronger positive than negative understanding of medication shown through higher reported need of medication than concerns about consequences of the use. Males reported higher negative understanding than females $(p<0.001)$. No difference between males and females was reported on BMQ subscale positive understanding. The patients who used medication for their mental illness reported higher positive understanding than those patients not using drugs for their mental illness $(p<0.001)$. No difference in negative understanding was found between patients using and not using drugs for their mental 
illness. No difference in negative or positive understanding of medication was reported between the in-patient and the out-patient population.

Conclusion: Some patient groups reported higher negative understanding of medication. These differences are important for the health care service in order to offer individually based treatment and follow up. It would be of interest to identify if the patients' understanding of medication changes over time.

Disclosure of Interest: None Declared.

HP-PC005: Hospital-acquired hyperkalaemia events in older patients: a frequent and multifactorial adverse drug reactions

L. Robert ${ }^{1, *}$, G. Ficheur ${ }^{1,2}$, B. Decaudin ${ }^{3,4}$, J. Gellens ${ }^{1}$, M. Luyckx ${ }^{5}$, R. Perichon ${ }^{1}$, S. Gautier ${ }^{6}$, F. Puisieux ${ }^{1,7}$, E. Chazard ${ }^{1,2}$, J.-B. Beuscart ${ }^{1,7}$

${ }^{1}$ EA 2694 - Santé publique: épidémiologie et qualité des soins, University of Lille $2,{ }^{2}$ Department of Public Health, ${ }^{3}$ Department of Pharmacy, CHU Lille, ${ }^{4}$ EA 7365 - Groupe de recherche sur les injectables et les technologies associées, University of Lille 2, Lille, ${ }^{5}$ Department of Pharmacy, CH Denain, Denain, ${ }^{6}$ Department of Pharmacology, ${ }^{7}$ Department of Geriatrics, CHU Lille, Lille, France

Background and objective: Hyperkalaemia is a frequent and severe adverse drug reaction (ADR) among older patients. At-risk situations of such ADRs cannot be accurately identified in hospital inpatients because community- and hospital-acquired hyperkalaemia events were mixed in most studies. Moreover, drugs and other risk factors were assessed separately. The objective was to accurately identify ADRs and to analyse the association between drugs and precipitating factors among hospital-acquired hyperkalaemia events, by older patients.

Design: We conducted a retrospective, observational, mono-centric study in a general hospital over a 4-year period. An automated detection identified hyperkalaemia events $(\geq 5.4 \mathrm{mmol} / \mathrm{l})$ occurring 3 days after hospital admission, amongst 9339 eligible hospital stays by patients aged 75 years or older. Two independent experts reviewed all hyperkalaemia events to adjudicate adverse drug reactions (Kramer's algorithm), determine inappropriate prescriptions (STOPP criteria), evaluate avoidability of hyperkalaemia event (Hallas criteria) and identify associated risk factors.

Results: The independent expert review confirmed 471 hyperkalaemia events in 421 hospital stays (4.5\% of hospital stays) and validated 379 $(80.5 \%)$ hyperkalaemia-ADRs among these hyperkalaemia events. A multifactorial cause (i.e. at least one drug with a precipitating factor or with another drug) was identified in 350 (92.3\%) hyperkalaemia-ADRs. Most of the hyperkalaemia-ADRs were avoidable (79.9\%), mainly in relation with a multifactorial cause. Only $19.5 \%$ of prescriptions involved in hyperkalaemia-ADRs were inappropriate.

Conclusion: Hospital-acquired hyperkalaemia events among older patients are most often multifactorial ADRs. Prevention of hyperkalaemia-ADRs need to focus more on precipitating factors of hyperkalaemia events.

Disclosure of Interest: None Declared.

HP-PC006: Defining an assessment and comparison method for the development of clinical pharmacy activities in Belgian hospitals

H. Yaras ${ }^{1, *}$, M. Claesen ${ }^{1}$, I. Vanden Bremt ${ }^{1}$, M. Haelterman ${ }^{1}$, J.-D. Hecq $^{2}$ on behalf of Advisory Working Group on Clinical Pharmacy, FPS Health, Belgium

${ }^{1}$ Federal Public Service Health, Brussels, ${ }^{2} \mathrm{CHU}$ UCL Namur, Yvoir, Belgium
Background and objective: Since 2014, the FPS Health grants a financial incentive to general hospitals and to four psychiatric institutions in order to sustain the development of clinical pharmacy that had been initiated through pilot projects $(0.25$ FTE pharmacist $/ 200$ beds/year). A strategic plan for 2015-2020 has been set up to support the hospitals in this approach. The evolution is monitored by the Working Group on Clinical Pharmacy of the FPS Health. The objective is to assess the development of clinical pharmacy activities in each hospital that receives funds.

Design: The 107 hospitals that receive funds completed and returned the questionnaire provided by the FPS Health on their activities in 2016. On the basis of collected information, a semi-quantitative analysis made it possible to assess different approaches by awarding scores to the different response categories. Three main sections were defined to make the assessment. The first section comprises strategy and communication items (management). The second section relates to activities targeted at healthcare providers and/or a group of patients (non-patient specific activities). Finally, the third section concerns activities carried out for a specific patient (patient specific activities). An overall score has been calculated for each hospital on the basis of the score obtained in those three sections.

Results: The median overall score for all hospitals is 57 points out of 100. One-third of the hospitals achieved a score above 60 points whereas one-third had a score below 50 points. The remaining third obtained a score between 50 and 60 points. Furthermore, the information collected has been used to draw up a map of the clinical pharmacy activities.

Conclusion: This assessment method has revealed the varying degrees of development of clinical pharmacy in Belgian hospitals. All the information has been gathered in an annual report and thanks to an anonymous code granted to each hospital, it is possible to compare the results obtained between the different institutions. This work shall be repeated within the framework of the strategic plan for providing support to the hospitals and for monitoring the evolution of clinical pharmacy.

Disclosure of Interest: None Declared.

HP-PC007: Providing a safe management of high alert medications in a manual unit dose drug dispensing system

I. Mariotti ${ }^{1}$, L. Quint ${ }^{2}$, P. Broumault ${ }^{3}$, G. Ghanem ${ }^{4}$, J. Khoury ${ }^{3}$, X. Bohand ${ }^{1 \text {,* }}$

${ }^{1}$ Pharmacy, ${ }^{2}$ Medicine, ${ }^{3}$ Quality, ${ }^{4}$ Nursing, American Hospital of Paris, Neuilly Sur Seine Cedex, France

Background and objective: In the framework of the accreditation by the Us Joint Commission (JC), the American Hospital of Paris (AHP) has to safely manage high-alert medications (H-AM). Chapter MM.01.01.01 of the JC Manual contains very specific H-AM recommendations. The objective of this study is to present the measures taken by the AHP to meet the requirement of these standards in our unit dose drug dispensing system (UDDS). The UDDS currently covers 130 beds, accounting for $66 \%$ of our overall capacity.

Design: A multidisciplinary working group has been established for this purpose. Procedures that we had in place were subsequently reviewed and revised according to the following:

- Results from analysis of the specific tools deployed for care professionals at the AHP, the safety audits targeting the management and use of $\mathrm{H}-\mathrm{AM}$, the analysis of adverse drug events and $\mathrm{H}-\mathrm{AM}$ errors and assessment of the H-AM policy compliance.

- Benchmarking with other hospitals.

Results: The working group proposed new specific measures and policies to the Medication Management committee that were 
unanimously adopted. Accordingly, the H-AM list and the global $\mathrm{H}-\mathrm{AM}$ policy were reviewed and revised. Yellow colour coding is now widely used to clearly differentiate the H-AM at the pharmacy and in each of the patient care units: each unit dose of H-AM is identified by a yellow sticker starting at the source in central pharmacy right up to the patient's bedside and patient administration. At the pharmacy, the H-AM doses are stored in a specific medicine cabinet. In the UDDS, a special review of medication orders by a pharmacist (level 2 of the French society of Clinical Pharmacy) is now required. For each dispensing dose of $\mathrm{H}$-AMed, a double check is carried out, first by a pharmacy technician during the dispensing step, and secondly by the nurse during the drug administration. With the exception of those dispensed in the UDDS, storage of H-AM doses is prohibited in the patient care units. Furthermore, non-administered $\mathrm{H}$-AM doses must be returned to the pharmacy. Lastly, specific mandatory training for health care professionals was provided for the safe and correct use of H-AM.

Conclusion: Preparation for the JC visit was a real opportunity for us to redefine and improve the management of H-AM at the AHP. In July 2017, the JC recognized the important efforts made by the AHP and validated all the measures implemented. This experience underlines the importance of collaborative teamwork in order to implement new procedures and new tools. The next step will be to carry out an assessment of the "new" H-AM management.

Disclosure of Interest: None Declared.

\section{HP-PC008: Management of delirium in elderly people: a randomized controlled trial}

\section{K. Iltingreuke ${ }^{1, *}$, A. Roos ${ }^{1}$, P. Ohrmann ${ }^{2}$, T. Duning ${ }^{1}$, G. Hempel ${ }^{3}$}

${ }^{1}$ Department of Neurology, ${ }^{2}$ Department of Psychiatry, University Hospital of Muenster, ${ }^{3}$ Department of Pharmaceutical and Medical Chemistry, Clinical Pharmacy, Westfälische-Wilhelms-University of Muenster, Muenster, Germany

Background and objective: Delirium is a common and severe condition. In particular for older patients it leads to cognitive impairments in every day functioning with substantial health care costs. The mortality is 20 -fold increased. Irreversible cognitive deficits are proven in $50 \%$ of cases.

The age, the cognition and the multimorbidity, combined with polypharmacy are the most predisposing risk factors to a delirium with $11-30 \%$ of cases being drug-induced.

Our university hospital established a multidisciplinary department, which developed pharmacological and nonpharmacological guidelines for diagnosis, prevention and treatment of delirium.

The primary objective of our open randomized controlled trial was to compare the effectiveness of multidisciplinary approaches to reduce the risk of delirium in surgical and nonsurgical patients aged 65 years and over.

Setting and method: From January 2016 to December 2017, 1472 patients aged 65 years and over were screened on admission by using the Montreal Cognitive Assessment (MoCA). A total of 936 patients $(64 \%)$ had an elevated risk for delirium (MoCA $<26$ points) and $84 \%$ (785) of these patients could be included and randomized.

The intervention group $(n=420)$ received our standardized treatments, like constant detection of delirium, specialized nursing and medication optimization by pharmacists. Whereas the control group $(n=383)$ was treated as usual without any standardized strategies.

The cognitive outcome of each patient was assessed by a second MoCA before discharge.

Main outcome measures: The rate of delirium and the duration of delirium in the two different groups.

Results: The risk of a manifest delirium during hospitalization was more than $50 \%$ higher in the control group, compared to the intevention group (20.4\% control group vs. $6.5 \%$ intervention group (OR $0.27,95 \%$ CI $0.17-0.44, p<0.001)$ ).

The duration of delirium in the intervention group was reduced by half, compared to the control group $(3.5$ days vs. 7 day $(p<0.001)$ ). Conclusion: The results of our trial demonstrate that not every delirium can be prevented, but the rate and the duration can be significantly reduced.

Furthermore, the results emphasize the importance of a multidisciplinary team consisting of nurses, neurologists and clinical pharmacists. The inappropriate use of non-evidence-based medication of delirium (e.g. inappropriate application of antipsychotics, benzodiazepines and anticholinergic substances) could be reduced by intensive training of medical staff and pharmaceutical counselling.

Considering the demographic change, we recommend implementation of a multidisciplinary approach for consistent and standardized management of delirium.

Disclosure of Interest: None Declared.

\section{Oral communication III: Innovations in pharmaceutical care}

HP-PC009: Impact of a pharmacist independent prescriber (PIP) on the haematology ward at the southern health and social care trust (Craigavon Area Hospital)

\section{Fox ${ }^{1, *}$, J. Agnew ${ }^{1}$, J. Palmer², K. Boyd ${ }^{2}$, B. Girvin ${ }^{3}$}

${ }^{1}$ Pharmacy Department, ${ }^{2}$ Haematology Department, Southern Health and Social Care Trust, Craigavon, NI, ${ }^{3}$ School of Pharmacy, Queen's University Belfast, Belfast, United Kingdom

Background and objective: The Southern Health and Social Care Trust has delivered services run by PIPs (pharmacist independent prescribers) for a number of years. Anticoagulation clinics were one of the first services to utilise the skills of PIPs and since then the drive for more PIPs has introduced prescribing by pharmacists in a number of diverse areas including haematology. This supports key services as the number of patients who are admitted to hospital with complex health issues is increasing every year. The aim of this study is to describe, quantify and evaluate prescribing undertaken by a pharmacist on the haematology ward and to describe the views of doctors, nurses and patients about pharmacist prescribing.

Design: The haematology pharmacist at Craigavon area hospital registered as a PIP with the trust in late 2016. In early 2017, data was collected for 6 months on the pharmacist's prescribing interventions. The prescribing interventions were collected weekly and analysed according to number and type of intervention and graded for improvements to patient care using the Eadon scale. The views of healthcare professionals and patients on pharmacist independent prescribing were also explored via questionnaires which were distributed on the last week of data collection.

Results: 410 prescribing interventions, all Eadon graded $\geq 4$, indicating improved patient care, were made by the PIP. These interventions would have had to be amended by a doctor after a request to do so by the pharmacist, thus releasing time for medical staff and improving patient safety and care. Most interventions were undertaken on admission and during inpatient stay. More than $50 \%$ of discharge letters clinically checked by the PIP needed prescribing interventions. All 24 doctors, nurses and patients surveyed supported this new role for pharmacists and found it beneficial to patient care and service provision.

Conclusion: The PIP on the haematology ward at Craigavon area hospital improved patient care and this was acknowledged by healthcare professionals and patients alike. PIPs have an important role as members of the wider healthcare team in improving medicines 
optimisation and care for patients. They should be fully integrated into future healthcare service and workforce strategies. Further development of pharmacist independent prescribing is of great importance and further studies on its benefits are needed. The future of pharmacist independent prescribing depends on new and innovative models of care as well as further research through audit and review.

Disclosure of Interest: None Declared.

DI001: Pilot on harmonizing dosing recommendation for term and preterm neonates in the Netherlands (NEODOSE project)

\section{A. de Hoop-Sommen ${ }^{1, *}$, T. M. van der Zanden ${ }^{2}$,} S. N. de Wildt ${ }^{3,4}$

${ }^{1}$ Medicine Information Centre, Royal Dutch Pharmacists Association (KNMP), The Hague, ${ }^{2}$ Department of Paediatrics, ${ }^{3}$ Intensive Care and Paediatric Surgery, Erasmus MC; Sophia Children's Hospital,

Rotterdam, ${ }^{4}$ Department of Pharmacology and Toxicology, Radboud University, Nijmegen, Netherlands

Background and objective: Scientific evidence on the use of a drug in preterm and term neonates is scarce or lacking. Therefore, every Neonatal intensive care unit (NICU) or high care unit in the Netherlands has its own local treatment protocols. Also, the Dutch Paediatric Formulary has not included dosing recommendations for all relevant drugs within the neonatal age range due to the lack of guidance from the scientific literature. Therefore, the Dutch Pediatric Formulary and the Dutch Neonatologists from all Dutch NICU's and high care units started a collaboration -the Neodose project- to elucidate the rationale for dosing recommendations for (pre)term neonates.

Design: We have learned from another project on dose adjustment in children with renal impairment that when evidence is scarce, we must rely on the consensus amongst healthcare professionals. To establish the consensus on neonatal dosing, we first investigated which drugs were used on neonatal wards, resulting in a list of 93 frequently used drugs. From this list the working group selected 14 drugs that were prioritized to elucidate within the context of the pilot. Every member of the Neodose working group collected their local dosing guidelines for preterm $(<37$ weeks of gestational age (GA)) and term ( $\geq 37$ weeks of GA) neonates. The local protocols of these drugs were collected and put together. Then a meeting was arranged to discuss the differences and the rationale for use to achieve consensus on the dosing recommendations.

Results: Not all NICU's had a protocol for every drug or indication. Sometimes a national guideline was available. The rationale for recommended dose within the treatment protocols or the national guideline was limited or unclear. The differences between the dosing advices concern for example the maximum dose, whether or not a loading dose is recommended, route of administration and frequency of dosing. For four indications, local protocols did not differ between NICU's. For the other indications there were 2-9 different protocols. The Neodose working group managed to achieve consensus on 33 dosing recommendations for 14 drugs with each 1-3 indications, most of the indications for both preterm and term neonates. The consensusbased dosing recommendations were different from the local treatment protocols in 13 out of 33 dosing recommendations.

Conclusion: Achieving national consensus about dosing advice for (pre)term neonates is feasible. The established consensus-based dosing advice will be published in the Dutch Children's Formulary. A further step is to crosscheck the established consensus-based dosing recommendations with available literature.

Disclosure of Interest: None Declared.
DI002: Selective digestive decontamination uses in French hospitals in 2018

\section{J.-M. Alili ${ }^{1, *}$, R. Rasamison ${ }^{1}$, M.-P. Berleur ${ }^{1}$, M.-C. Husson ${ }^{1}$}

${ }^{1}$ Pharmaceutical and Medical Department, Pharmaceutical Establishment of Paris hospitals group, AP-HP, Paris, France

Background and objective: Selective digestive decontamination (SDD) aims at preventing or eradicating intestinal carriage of pathogenic bacteria.

Since 2010, our establishment has developed a hospital preparation of colistin-gentamicin in capsule for SDD (COLI-GENTA), available at dosing $27-50 \mathrm{mg}$ for children and 135-100 $\mathrm{mg}$ for adults.

The aim of this study was to re-evaluate the usefulness of COLIGENTA in French hospitals.

Setting and method: The survey was conducted from November 2017 to March 2018 in 58 French hospitals, customers of COLIGENTA from 2014 to 2017. Pharmacists and clinicians from ICU, Haematology, Gastroenterology and Infectious diseases wards were contacted by e-mail and telephone.

Main outcome measures: Pharmacists and clinicians were interviewed on the use of COLI-GENTA in their hospital: indication, posology and profile of treated patients.

Results: Thirty/58 hospitals answered the questions: 30 pharmacists/8 clinicians.

Three types of wards used COLI-GENTA in children and adults:

- Haematology, for transplanted/neutropenic patients: 3-8 capsules/ $\mathrm{d}$ for several weeks.

- ICU, for patients undergoing transplantation, bloodstream infection, polytrauma or serious burns: 4 capsules/d during 3-4 weeks.

- Gastroenterology, for patients undergoing colorectal surgery or bacterial multiplication: 1-4 capsules/d for 1 day to several weeks.

Eleven hospitals indicated they stopped SDD use due to antimicrobial resistance (AMR) issues.

Clinical studies about SDD are numerous but results are difficult to compare given the huge variability of protocols, differences in ecological environments and methodology bias.

SDD can decrease ventilator-associated pneumonia (VAP) in ICU patients and could prevent organ dysfunction in ill burn patients, but longterm ecological effects are unknown and could lead to AMR, which has become a big threat to global health as recalled by United Nations in 2016.

Concerning colistin-gentamicin:

- WHO recently reminded that resistance to colistin, which is the last resort treatment against specific bacteria, has been detected in several countries, making infections caused by such bacteria untreatable. WHO also highlighted the importance of colistin and gentamicin in its list of essential medicines.

- European centre for disease prevention and control recently underlined that in countries with high levels of AMR, colistin is among the only few therapeutic options available and considered AMR to colistin as a serious warning.

In 2017, among European specialists, SDD was considered to be used where the prevalence of multidrug-resistant bacteria is low, as taken up by several French ICU anaesthetists societies.

Conclusion: To assess the usefulness of COLI-GENTA for SDD, it is necessary to take into account:

- the diversity of drug combinations currently used for SDD in hospitals

- the potential epidemiological consequences of SDD use

- the results of clinical studies, which display methodological biases.

According to this survey and to clinical experience, COLI-GENTA remains suitable in ICU to prevent VAP and in haematology in seriously ill patients, considering an ecological context.

Disclosure of Interest: None Declared. 
PE001: Assessment of patient adherence to long term medicines within a large cohort study using the dried blood spot technique

\section{F. J. Jirjees ${ }^{1, *}$, G. Chen ${ }^{1}$, J. C. Mcelnay ${ }^{1}$ \\ ${ }^{1}$ School of Pharmacy, Queen's University Belfast, Belfast, United Kingdom}

Background and objective: Although pharmacoepidemiological studies, based on prescribing datasets, provide useful data on the effectiveness and risk of medicine use, one of the main obstacles to drawing meaningful conclusions from this type of study is medication non-adherence, which is a major issue in the management of chronic illness. It is well known that approximately $50 \%$ of medicines prescribed in primary care are not taken by patients as recommended by prescribers, with many medicines not taken at all by patients. The aim of the present research was to develop and use a novel direct method to assess medication exposure/adherence in a large cohort study being conducted in Northern Ireland.

Setting and method: Dried blood spot (DBS) samples were collected on Guthrie cards from participants as they joined the study. Participants $(\mathrm{n}=805)$ who were being treated with one of the following medicines form the sample for the present research: metformin, allopurinol, fluoxetine, bisoprolol, amlodipine and methotrexate. All participants were $\geq 50$ years old. DBS based analytical methods were developed for the six drugs and/or their active metabolites. Fixed volume $(15 \mu \mathrm{l})$ DBS samples were used throughout for assay development and for patient samples. Simple solvent extraction approaches were used for four medicines, while solid phase extraction was used for two medicines. In all cases reversed phase HPLC was utilised with either UV (metformin and allopurinol), fluorescence (fluoxetine and bisoprolol) or mass-spectrometry (amlodipine and methotrexate) detection.

Main outcome measures: Medication exposure/adherence in a large cohort study being conducted in Northern Ireland using dried blood spot technique.

Results: Linear calibration curves were obtained over wide concentration ranges for each of the six medicines, including at levels many times lower than expected steady state trough levels and higher than expected steady state peak levels of the medicines of interest after multiple dosing. All assay methods were shown to have good selectivity, specificity, accuracy and precision according to international guidelines. A significant proportion of participants $(12.7 \%)$ within the cohort had no medication of interest in their blood samples, clearly indicating that they were not taking any of the medication at the time of sampling. This was a particular issue for patients using bisoprolol and fluoxetine, i.e. $20.6 \%$ and $23.2 \%$ of participants respectively. Only $63.3 \%$ of the patients overall had blood levels within what would be considered the effective therapeutic range of the medicines studied.

Conclusion: The results of the present study illustrate, the possibility of using a DBS sampling approach to objectively assess adherence/exposure to medicines within large cohort studies. Knowledge of medicine exposure using this approach will enhance the precision of conclusions drawn from pharmacoepidemiological studies, rather than depending on prescribing data alone.

Disclosure of Interest: None Declared.

PEC001: Pharmacoeconomic evaluation of clinical pharmacists' interventions at a University Teaching Hospital in Ireland

A. O'Connor ${ }^{1}$, A. Harnett ${ }^{1}$, S. Byrne ${ }^{2, *}$

${ }^{1}$ Pharmacy Department, University Hospital Limerick, Limerick, ${ }^{2}$ School of Pharmacy, UNIVERSITY COLLEGE CORK, Cork, Ireland

Background and objective: Clinical Pharmacy interventions are an integral part of a clinical pharmacists' job. Pharmacist interventions can be associated with many positive outcomes for both the patient and the healthcare provider. This includes prevention of adverse drug events (ADEs). ADEs are associated with a huge economic burden and therefore, potentially, clinical pharmacy services could lead to significant cost savings to the healthcare provider. Ireland's healthcare budget is under ever increasing pressure and therefore healthcare providers need to evaluate the service they are providing and ask if they provide a service that offers value for money. The aim of this study was to calculate the cost avoidance generated by the interventions pharmacists made at a large University Teaching Hospital due to the prevention of ADEs.

Setting and method: This study was undertaken at a University Affiliated hospital with 428 in-patient beds. A data collection form was designed and piloted for the purpose of this study. Interventions were recorded, using the data collection form by a group of pharmacists over a 14 day period between 27th Feb 2017 and 16th Mar 2017. Interventions were then assigned a rating score based on the likelihood of an ADE occurring, if the intervention had not been made. These scores were then used to calculate the cost avoidance and net cost-benefit of the interventions. A cost-benefit ratio was also calculated.

Main outcome measures: Cost-benefit ratio and cost avoidance of ADEs were calculated.

Results: A total of 729 interventions were recorded over the 14 days. These interventions generated a cost avoidance of $€ 128,658$. The cost of providing the pharmacy service was calculated to be $€ 12,583$ for the same time period. This resulted in a net cost-benefit of $€ 116,075$ and a net cost-benefit ratio of 9.2:1. Basic grade pharmacists were found to be more cost effective than senior grade pharmacists with a cost-benefit ratio of 14.7:1 vs. 5.7:1 respectively. The most common intervention type related to medicine omissions at the point of admission $(22.9 \%)$.

Conclusion: This study has shown that there is substantial cost avoidance to the healthcare provider achievable through the implementation of clinical pharmacy services. This study also highlighted the importance of medicine reconciliation in preventing ADEs. Disclosure of Interest: None Declared.

PEC002: Cost-effectiveness of pharmacist care for managing patients at high risk for cardiovascular disease in Canada

\section{Y. N. Al Hamarneh ${ }^{1, *}$, K. Johnston ${ }^{2}$, C. Marra ${ }^{3}$, R. Tsuyuki ${ }^{1}$}

${ }^{1}$ University of Alberta, Edmonton, ${ }^{2}$ Broadstreet HEOR, Vancouver, Canada, ${ }^{3}$ University of Otago, Dunedin, New Zealand

Background and objective: The $\mathrm{R}_{\mathrm{x}} \mathrm{EACH}$ randomized trial demonstrated that community pharmacist prescribing and care reduced the risk for cardiovascular $(\mathrm{CV})$ events by $21 \%$, compared to usual care.

Objective: To evaluate the economic impact of pharmacist prescribing and care for $\mathrm{CV}$ risk reduction in a Canadian setting.

Setting and method: A Markov cost-effectiveness model was developed to extrapolate potential differences in long-term CV outcomes, using different risk assessment equations. The mean change in $\mathrm{CV}$ risk for the two groups of $\mathrm{R}_{\mathrm{x}} \mathrm{EACH}$ was extrapolated over 30 years, with costs and health outcomes discounted at $1.5 \%$ per year. The model incorporated health outcomes, costs and quality of life to estimate overall cost-effectiveness. It was assumed that the intervention would be $50 \%$ effective after 10 years. Individual-level results were scaled up to population level based on published statistics (29.2\% of Canadian adults are at high risk for CV events). Costs considered included direct medical costs as well as the costs associated with implementing the pharmacist intervention. Uncertainty was explored via probabilistic sensitivity analysis. 
Main outcome measures: impact of pharmacist prescribing and care for $\mathrm{CV}$ risk reduction on healthcare cost, quality adjusted life years and $\mathrm{CV}$ events.

Results: It is estimated that the Canadian healthcare system will save $\$ 4.6$ billion over 30 years, if the pharmacist intervention was delivered to $15 \%$ of the eligible population. Pharmacist care would be associated with a gain of 592,049 Quality Adjusted Life Years and avoid more than 9 million CV events. Those savings can reach $\$ 13.8$ billion over 30 years if the intervention was delivered to $45 \%$ of the eligible population. In that case, Pharmacist care would be associated with a gain of 1,776,146 Quality Adjusted Life Years and avoid more than 27 million $\mathrm{CV}$ events.

The intervention is economically dominant, i.e. it is both more effective and reduces costs when compared to usual care.

Conclusion: Across a range of one-way and probabilistic sensitivity analyses of key parameters and assumptions, pharmacist prescribing and care is both more effective and cost-saving compared to usual care. Canadians need, and deserve such care.

Disclosure of Interest: None Declared.

PT002: Review of available mobile applications to support diabetes self-management: preliminary findings

M. Correia ${ }^{1}$, A. Henriques ${ }^{2}$, A. P. Cláudio ${ }^{3}$, A. Mendes ${ }^{2}$, A. Cavaco ${ }^{4}$, M. B. Carmo ${ }^{5}$, M. P. Guerreiro ${ }^{2,6, *}$

${ }^{1}$ Instituto Universitário Egas Moniz, Monte de Caparica, ${ }^{2}$ Unidade de Investigação \& Desenvolvimento em Enfermagem (ui\&de), Escola Superior de Enfermagem de Lisboa, ${ }^{3}$ BioISI (Biosystems \& Integrative Sciences Institute), Faculdade de Ciências Universidade de Lisboa, ${ }^{4}$ Faculdade de Farmácia Universidade de Lisboa, ${ }^{5}$ BioISI (Biosystems \& Integrative Sciences Institute), Faculdade de Ciências da Universidade de Lisboa, Lisboa, ${ }^{6}$ Centro de Investigação Interdisciplinar (CiiEM), Instituto Universitário Egas Moniz, Monte de Caparica, Portugal

Background and objective: Many people with diabetes have difficulties in adhering to daily treatment, which is key to optimal glycaemic control. Commercial mobile applications (apps) developed to support diabetes self-management have been described by several reviews. However, the app market is dynamic, making it difficult to stay updated about the available options. The aim of this study is to review mobile apps to support diabetes self-management.

Setting and method: The Google Play Store was searched using the keyword "diabetes" in February 2018. The retrieved apps were listed in a database derived from the literature. Data for each app were extracted from the information provided by the Google Play Store, the app website and the app itself. Exclusion criteria included apps targeting healthcare professionals, apps with a non-therapeutic purpose, apps uniquely in languages not mastered by the research team, and apps unavailable due to $\log$-in errors. Statistical analysis was performed with the aid of SPSS v. 25, excluding missing values.

Main outcome measures: General characteristics of apps. Nature of available features.

Results: A total of 249 apps were retrieved from Google Play Store. Data extraction is on-going; we report preliminary findings on 123 apps. About two-thirds of the apps were released in the last 3 years and, on average, their last update was carried out 15 months ago (SD 18). About half of the apps were exclusive for the Android operating system $(n=67 ; 54.5 \%)$; the remaining ran also on additional systems. There was a predominance of apps providing full access at no cost $(n=93 ; 75.6 \%)$. About half of the apps $(49.6 \%)$ were below the 5000 downloads cut-off. On average, apps received a score of 4.1 (SD 0.6) out of 5 from users. More than $80 \%$ of apps targeted patients only $(\mathrm{n}=105 ; 85.4 \%)$. Connectivity for wearables and other devices was infrequent $(\mathrm{n}=21 ; 17.1 \%)$; when present, it pertained mostly to glucometers. Sixty-four apps were reviewed in-depth for the features available. The most common features were information, documentation, data analysis, reminder/alarm and data forwarding $(34.1,31.7$, $29.3,23.6$ and $22.8 \%$, respectively). Only a minority provided counselling on treatment based on users' input $(n=12 ; 9.8 \%)$.

Conclusion: Apps varied in the range of features provided. The next step is to analyse the usability of a subset of apps in relation to specific populations, such as older people with type 2 diabetes. This may enable pharmacists and other health professionals to personalise care in response to specific patient needs.

Disclosure of Interest: None Declared.

PH001: Quality of prescribing for Norwegian elderly patients using automated multidose dispensed drugs

A. V. Josendal ${ }^{1,2, *}$, A. G. Granas ${ }^{2}$, T. S. Bergmo ${ }^{1}$

${ }^{1}$ Norwegian Centre for E-healthresearch, Troms $\emptyset,{ }^{2}$ School of pharmacy, University of Oslo, Oslo, Norway

Background and objective: Multidose drug dispensing (MDD) is an adherence aid that provides patients with machine-dispensed medicines in disposable unit bags, usually for a 14 day period. Every time for medicine administration, i.e. morning, midday or evening, the plastic unit bag is opened by patients or health personnel. The alternative to MDD are manual dosettes, usually dispensed for 1 week at a time. Previous studies have suggested that the quality of prescribing, with time, is lower for MDD users, compared to patients receiving regular prescriptions.

The aim of this study was to examine the proportion of inappropriate medication use in Norwegian elderly patients receiving MDD.

Setting and method: Patients in Norway, $\geq 65$ years, receiving MDD. Cross-sectional study comprising 68499 patients receiving home care services $(n=48,316)$, living in nursing homes $(n=17,539)$ or home-dwelling $(n=2644)$. The quality of drug therapy was assessed using the Norwegian General Practice Criteria (NORGEP) and drug-drug interactions was investigate using the Norwegian Medicines Agency database.

Main outcome measures: Number of prescribed drugs, potentially inappropriate medications and drug-drug interactions.

Results: Patients receiving home care services were prescribed on average 6,6 drugs $(\mathrm{SD}=3.0)$, patients in nursing homes were prescribed 5,8 drugs $(\mathrm{SD}=3.0)$ and home-dwelling patients were prescribed 7.0 drugs $(\mathrm{SD}=2.7)$ Twenty percent used at least one potentially inappropriate medication, $21 \%$ in nursing homes, $20 \%$ in home care, and $18 \%$ home-dwelling patients $(p<0.001)$. Concomitant use of three or more psychotropic drugs was the most commonly identified inappropriate prescribing in all three groups. Drug-drug interactions were identified in $12 \%$ of home dwelling patients, $10 \%$ in nursing home, and $13 \%$ in home care service.

Conclusion: About one-fifth of the elderly Norwegian patients using MDD were exposed to potentially inappropriate medications. These patients should be targeted for interprofessional medication reviews to prevent side effects.

Disclosure of Interest: None Declared.

\section{Poster discussion forum I: Community pharmacy}

CP-PC007: A systematic scoping review of interventions to support evidence-based prescribing of oral nutritional supplements in primary care

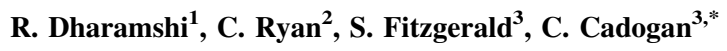


${ }^{1}$ School of Medicine, Royal College of Surgeons in Ireland, ${ }^{2}$ School of Pharmacy and Pharmaceutical Sciences, Trinity College Dublin,

${ }^{3}$ School of Pharmacy, Royal College of Surgeons in Ireland, Dublin, Ireland

Background and objective: Malnutrition is an important clinical condition, particularly amongst the older population. The implications of malnutrition are considerable, and the associated costs are high (e.g. increased morbidity, mortality and healthcare resource utilisation). Strategies to treat malnutrition include oral nutritional supplements (ONS). Inappropriate ONS prescribing whereby ONS are prescribed without nutritional assessment is common (prevalence estimates range between 30 and 70\%) and has significant cost implications. This review's objective was to provide an overview of evaluations of interventions aimed at supporting evidence-based ONS prescribing in primary care.

Setting and method: A systematic scoping review was undertaken using relevant methodological guidance. PubMed and EMBASE were searched from inception to June 2017. Search terms included malnutrition and ONS. In order to meet inclusion criteria, studies had to: evaluate interventions targeting ONS prescribing in primary care; use a comparative evaluation (e.g. control group, before/after design); be published in the English language. All outcomes for included studies were documented. Two review authors independently screened abstracts and extracted data from included studies using a purposefully-designed data extraction form that enabled presentation of a logical and descriptive summary of findings. Results were summarised using narrative synthesis.

Main outcome measures: The review was led by a number of questions focussing on key issues such as the types of evaluated interventions, study designs and outcomes reported in the current literature.

Results: 2627 records were screened for inclusion and eight studies were included in the review. All studies followed an uncontrolled before-and-after design. Interventions ranged from dietician assessment of prescriptions to policy-level changes involving complete transfer of ONS prescribing privileges to dieticians. Post-intervention study results reported improvements in ONS prescribing as measured by reductions in prescribing levels and a number of studies highlighted potential cost savings associated with reductions in inappropriate ONS prescribing.

Conclusion: This review provides the first detailed overview of interventions aimed at improving evidence-based prescribing of ONS in primary care. The included studies were heterogeneous in terms of interventions and outcome assessments. Reporting of interventions was often poor and use of controlled experimental design was lacking. Robust evaluations of systematically developed interventions are needed in this area.

Disclosure of Interest: None Declared.

\section{CP-PC008: Medication discrepancies in patients with chronic} heart failure

\section{P. M. Schumacher ${ }^{1, *}$, N. Griese-Mammen ${ }^{1}$, L. Botermann ${ }^{1}$, U. Laufs ${ }^{2}$, M. Schulz ${ }^{1,3}$}

${ }^{1}$ Department of Medicine, ABDA - Federal Union of German Associations of Pharmacists, Berlin, ${ }^{2}$ Department of Cardiology, University Hospital, Leipzig University, Leipzig, ${ }^{3}$ Department of Clinical Pharmacy and Biochemistry, Institute of Pharmacy, Freie Universitaet Berlin, Berlin, Germany

Background and objective: that are treated by independent physicians. In addition to prescribed drugs $(\mathrm{Rx})$, selfmedication is common and further increases the high daily number of medications. We aimedt o quantify and characterize potential discrepancies between drugs documented by physicians and patients' actual medication use.

Design: As part of the randomized controlled trial (RCT) PHARMCHF, community pharmacists conducted a medication review type $2 \mathrm{a}$ with CHF outpatients $\geq 60$ years in the intervention group. Patients' study physician (general practitioner, ambulatory- or hospital-based cardiologist) provided information on the current medication. Here, we compared the medicines documented by the physician with the medication the patient stated to take during the brown bag interview (BBI) and classified discrepancies.

Results: Of $97 \mathrm{CHF}$ patients (mean $74.0 \pm 6.6$ years, $38.1 \%$ female), receiving a median of 9 (IQR 7-12) drugs, $83.5 \%$ had at least one discrepancy. Of 988 drugs examined, $36.0 \%$ were associated with at least one discrepancy. The most frequent discrepancy was omission of a drug (39.3\%), mostly involving Rx that the study physician was unaware of $(71.4 \%)$. Drugs not found in the BBI were responsible for $26.1 \%$ of discrepancies. Furthermore, patients' statements during the BBI revealed discrepancies in frequency of intake $(13.5 \%)$, units $(19.1 \%)$, time of intake (7.6\%), and drug strength (8.1\%). 482 of the 988 medicines $(48.8 \%)$ belonged to the ATC code 1st level C (cardiovascular (CV) system). $61.9 \%$ of all patients, had at least one discrepancy regarding their CV medication $(30.3 \%)$. In $67.1 \%$ of the $\mathrm{CV}$ drugs with discrepancies, agents acting on the renin-angiotensin system (C09), beta-blockers (C07), mineralocorticoid receptor antagonists (C03D), or diuretics (C03A-C) were involved.

Conclusion: Even within the context of an RCT, BBI revealed numerous and diverse discrepancies compared to physicians' documentation in the great majority of elderly outpatients with CHF. Deviations from prescribed drug regimens have the potential to compromise both patient safety and effectiveness of pharmacotherapy. A structured process, as tested in our study, is able to provide a complete overview of patients' entire drug regimen.

Disclosure of Interest: None Declared.

PT003: Preferences of patients with rheumatoid arthritis regarding disease modifying anti-rheumatic drugs: a discrete choice experiment

M. van Heuckelum ${ }^{1, *}$, E. Mathijssen ${ }^{2}$, M. Vervloet $^{3}$, A. Boonen ${ }^{4}$, R. Hebing ${ }^{5}$, A. Pasma ${ }^{6}$, H. Vonkeman ${ }^{7}$, M. Wenink ${ }^{2}$, B. van den Bemt $^{1}$, L. van Dijk ${ }^{3}$

${ }^{1}$ Rheumatology and Pharmacy, ${ }^{2}$ Rheumatology, Sint Maartenskliniek, Nijmegen, ${ }^{3}$ Nivel, Utrecht, ${ }^{4}$ Rheumatology, MUMC + , Maastricht, ${ }^{5}$ Rheumatology, Reade, Amsterdam, ${ }^{6}$ Rheumatology, Erasmusmc, Rotterdam, ${ }^{7}$ Rheumatology, MST, Enschede, Netherlands

Background and objective: Adherence to disease modifying antirheumatic drugs (DMARDs) in rheumatoid arthritis (RA) patients varies from 30 to $90 \%$, which may be partly due to trade-offs patients make regarding treatment benefits and drawbacks. Tailoring treatment options to these trade-offs might (implicitly) motivate patients to adhere to their drugs. The aim of this study is to identify subgroups in RA patients based on their preferences towards DMARD characteristics, and to identify characteristics associated with subgroup membership.

Setting and method: A discrete choice experiment based on a literature review, expert recommendations and focus groups was used to elicit preferences. In this multicentre study, patients were asked to state their choice over two different hypothetical treatment options, described by seven DMARD characteristics and three levels within each characteristic (e.g. route of administration: oral, subcutaneous and intravenous). Inclusion criteria were: RA according to the ACR/ EULAR2010 criteria, using at least one DMARD, and aged $\geq 18$ years. Latent class analysis and multinomial logistic regression were used to identify subgroups and characteristics (beliefs about 
medicines, patient- and disease-related variables) associated with subgroup membership.

Main outcome measures: Identification of subgroups with different preferences (including segment sizes) and characteristics associated with subgroup membership (expressed as relative risk ratios with 95\% CIs).

Results: Among 325 participating RA patients, three subgroups with the following segment sizes were identified: an administration-driven subgroup (45.6\%), a benefit-driven subgroup (29.7\%), and an indifferent subgroup (24.7\%). In the administration-driven subgroup, the oral route of administration was strongly preferred over subcutaneous and intravenous administration. Current bDMARD use (RRR: 0.50, 95\% CI 0.28-0.89) was significantly associated with assignment to the administration-driven subgroup, and high educational level (RRR: 11.4, 95\% CI 0.97-133.6) with assignment to the benefit-driven subgroup (indifferent subgroup as base scenario). Patients' concern beliefs did not contribute significantly to subgroup membership, whereas a near significant association between patients' necessity beliefs and membership of the benefit-driven subgroup was found (RRR: $1.12,95 \%$ CI $1.00-1.23, P=0.055$ ).

Conclusion: Three subgroups with different preferences were identified. Integrating these preferences in medication treatment decisions may improve adherence. Discriminating patients based on their beliefs about medicines, and patient and clinical characteristics is complex.

Disclosure of Interest: M. van Heuckelum Grant/Research support from Pfizer, E. Mathijssen Grant/Research support from Pfizer, M. Vervloet Grant/Research support from Pfizer, A. Boonen: None Declared, R. Hebing: None Declared, A. Pasma: None Declared, H. Vonkeman: None Declared, M. Wenink: None Declared, B. van den Bemt Grant/Research support from Pfizer, L. van Dijk Grant/Research support from Pfizer.

CP-PC009: Impact of GP practice-based pharmacist interventions on medication related problems and medication appropriateness

N. F. Syafhan ${ }^{1, *}$, S. Al Azzam ${ }^{1}$, M. Scott ${ }^{2}$, G. Fleming ${ }^{2}$, A. Hogg ${ }^{2}$, C. Scullin ${ }^{2}$, J. McElnay ${ }^{1}$

${ }^{1}$ School of Pharmacy, Queen's University Belfast, Belfast, Northern Ireland, ${ }^{2}$ Medicines Optimisation Innovation Centre, Northern Health Social Care Trust, Antrim Area Hospital, Northern Ireland, United Kingdom

Background and objective: Changing demographics across the UK has led to general medical practitioners (GPs) managing increasing numbers of older patients with multi-morbidity and resultant polypharmacy. Through government led initiatives within the National Health Service (NHS), an increasing number of GP practices employ pharmacist support. The objective of the present research was to assess the impact of GP practice-based pharmacist services on medication related problems and medication appropriateness.

Setting and method: A prospective, multi-centre, randomised, controlled intervention study in four different regions of the UK, involving patients across eight participating GP practices, with a 6 month follow-up period. Patients were randomly assigned to receive normal care or pharmacist supplemented care (focusing on medication optimisation).

Main outcome measures: The outcome measures utilised included number of medication related problems (MRPs) and medication inappropriateness (measured using the medication appropriateness index; MAI).

Results: A total of 356 adult patients (175 control and 181 intervention) were recruited. To date full data for assessing MRPs are available in 108 intervention patients who had three pharmacist faceto-face contacts. A total 346 MRPs were identified at baseline and 83 MRPs at 6 months. The most common sub-categories of MRPs related to dosage regimen, actual/potential adverse drug reactions and unnecessary drug therapy. Median data were 3 MRPs at baseline and 1 at 6 months $(p<0.001)$. Full data for MAI scoring to date has been performed on a random sample of 44 control and 43 intervention patients. A decrease in median score of inappropriate use per drug (from 0.80 to $0.57 ; p=0.003$ ) and per patient (from 8 to $4, p=0.009$ ) were noted in the intervention group. The most common criteria relating to inappropriateness were drug indications, dosage and administration directions. Control group patients had similar scores at baseline and end of study $(P>0.05)$.

Conclusion: The quality of medication use, as measured by incidence of MRPs and medication inappropriateness, was improved in patients who received GP practice-based pharmacist services.

Acknowledgement: Thanks to pharmacists who participated, and to the Association of the British Pharmaceutical Industry and the Indonesia Endowment Fund for Education for financial support.

Disclosure of Interest: None Declared.

CP-PC010: Knowledge and attitudes of women under treatment of osteoporosis at community pharmacy setting

O. Ozkan ${ }^{1, *}$, O. Gulec ${ }^{1}$, B. Torun ${ }^{1}$, S. L. Soylemez ${ }^{1}$, M. Sancar ${ }^{1}$, B. Okuyan ${ }^{1}$

${ }^{1}$ Department of Clinical Pharmacy, Marmara University Faculty of Pharmacy, Istanbul, Turkey

Background and objective: The aim of this study is to assess knowledge and attitudes of women who have received osteoporosis treatment at least a month.

Setting and method: The study was carried out at community pharmacy during 4 months [MS1] (20 December 2017-20 April 2018).

Main outcome measures: Turkish translation of the Beliefs about Medicines Questionnaire (BMQ-T) and medication adherence scale (Morisky, Green and Levine) were administered to assess patients' medication attitude and belief. Osteoporosis Health Belief Scale, Osteoporosis Self-Efficacy Scale and Osteoporosis Knowledge Test were also performed to evaluate patients' knowledge and attitude of osteoporosis.

Results: Among total of 100 individuals, the mean of age was calculated as $65.96 \pm 9.36(48.00-89.00)$. Of them, $44.0 \%$ had polypharmacy and $45.0 \%$ were adherent to their treatment. It was detected that patients adherent to their medications had higher score of osteoporosis knowledge test than patients with no medication adherent $(p<0.05)$. As the age of patient increase, a statistically significant increase was observed in the total score of osteoporosis knowledge test and the score of self-efficacy scale $(p<0.05)$. [MS1] An increase was observed in the concerns about medications of patients with a decrease in the level of knowledge about osteoporosis and an increase in the number of chronic illnesses $(p<0.05)$. Also, there was a statistically significant increase in the score of self-efficacy scale with an increase in the level of knowledge about osteoporosis $(p<0.05)$.

Conclusion: Increasing the knowledge level of patients about osteoporosis is positively associated with both patients' medication adherence and self-efficacy, furthermore it is related with decreased concerns about medication treatment. Thus, pharmacist should take responsibility of patients' education about osteoporosis to enhance their knowledge especially on early ages.

Disclosure of Interest: None Declared. 
CP-PC011: Community pharmacist assessment, management and advice giving to patients with asthma: a simulated study

S. Hasan ${ }^{1, *}$

${ }^{1}$ Clinical Sciences, Ajman University, Ajman, United Arab Emirates

Background and objective: Research has shown that there is an increase in the global prevalence of asthma. Pharmacists are well positioned to screen and refer patients for better management of asthma. This study aimed to evaluate pharmacists' ability to assess the 3 C's (Control, Compliance, Complications) and offer Management and Advice for patients with asthma in the United Arab Emirates.

Setting and method: Community pharmacies in the UAE were visited by three pharmacy students who role played a simulated patient requesting symptom relief from uncontrolled asthma without prescription. The scenario was based on predetermined plots to ensure standardization of pharmacist-patient communication.

Main outcome measures: Incidence of cough syrup and albuterol/ salbutamol inhaler supply, physician referral rate, correction of inhaler technique, counselling on asthma and its triggers, and counselling on the medications, adherence to the medications and adverse drug reactions were calculated.

Results: One hundred, ninety-five pharmacies were visited, $27 \%$ of pharmacists asked about the need for cough syrup and $60 \%$ asked about the need for albuterol inhaler. Only $26 \%$ of pharmacists asked about other medications and only one pharmacist asked about regular use of preventer medications and if the patient was adhering to them. Less than $20 \%$ assessed inhaler technique, and only $16 \%$ asked about side effects from medications. Most pharmacists (67\%) supplied at least one of the medications, however, only $4 \%$ of pharmacists correctly supplied albuterol, while $65 \%$ correctly referred the patient to a physician. One-third of pharmacists counselled the patient on the medications with only one giving complete information while $21 \%$ gave information about correct inhaler technique, the majority being incomplete.

Conclusion: This study revealed several shortcomings in the ability of pharmacists to assess control, management and advice giving relating to asthma, and the medications and devices that treat it. The study, however, reported a high rate of reliever (albuterol) supply and referral to a physician, as part of the correct management of patients with asthma by community pharmacists. This research has informed pharmacist education and training needs, which will help promote quality, systematic and consistent management of asthma by pharmacists.

Disclosure of Interest: None Declared.

CP-PC012: Effect of multidose drug dispensing on the time in therapeutic range in patients using vitamin $K$ antagonists: a randomised controlled trial

B. Mertens ${ }^{1,2, *}$, H.-F. Kwint ${ }^{1}$, S. Belitser ${ }^{2}$, F. V. D. Meer ${ }^{3}$, R. V. Marum ${ }^{4}$, M. Bouvy ${ }^{1,2}$

${ }^{1}$ SIR Institute for Pharmacy Practice and Policy, Leiden, ${ }^{2}$ Division of Pharmacoepidemiology and Clinical Pharmacology, Utrecht Institute for Pharmaceutical Sciences, Utrecht, ${ }^{3}$ Department of Thrombosis and Haemostasis, Leiden University Medical Centre, Leiden, ${ }^{4}$ Department of General Practice and Elderly Care Medicine and EMGO Institute for Health and Care Research, VU University Medical Centre, Amsterdam, Netherlands

Background and objective: A significant proportion of patients using vitamin $\mathrm{K}$ antagonists (VKA) have a low proportion of time in therapeutic range (TTR). One possible explanation of a low TTR is inadequate medication adherence to the complex dosing regimens of vitamin $\mathrm{K}$ antagonists. We aimed to improve the medication adherence, and thereby the TTR, by dispensing VKAs via automated multidose drug dispensing (MDD).

Setting and method: A RCT in 18 community pharmacies located in the catchment area of the Leiden Anticoagulation Clinic in the Netherlands. Patients over 65 years of age, VKA use over 9 months and a TTR $<65 \%$ were selected by the anticoagulation clinic. Screening on polypharmacy and inclusion was performed by the pharmacist. Randomisation was stratified on MDD use at baseline. Patients in the intervention group received all their drugs, including VKA, via a MDD system. Controls received VKA by manual dispensing. The primary outcome was analysed using a mixed-effects model with the intervention, TTR at baseline, and MDD at baseline as covariates and pharmacy as random effect.

Main outcome measures: The difference in TTR between the 6 months before- and during the study period.

Results: 179 patients were included. Mean age was $80.0 \pm 6.9$ years, $53 \%$ of the patients were men. Mean TTR during the study was $79.2 \pm 18.0$ (at baseline $60.8 \pm 16.4 \%$ ) in the intervention group and $72.5 \pm 20.1 \%$ (at baseline $55.9 \pm 18.0 \%$ ) in the control group. The intervention resulted in a $5.6 \%$ additional TTR increase compared to the control group $(p=0.048)$. At the moment of inclusion $63(35 \%)$ of the patients had a TTR $>65 \%$. Secondary analysis without these patients resulted in a mean TTR during the study of $78.6 \pm 18.8$ (at baseline $50.7 \pm 10.7$ ) in the intervention group and $69.0 \pm 20.6 \%$ (at baseline $46.3 \pm 13.1$ ) in the control group. This resulted in a $7.7 \%$ additional TTR increase compared to the control group $(p=0.034)$. No differences were seen in the number of bleedings.

Conclusion: Anticoagulation therapy in patients with a low TTR can be improved by dispensing the VKA via a MDD system.

Disclosure of Interest: None Declared.

\section{DI003: Fentanyl transdermal patch: are patients using it} correctly?

M. Viñas-Bastart ${ }^{1, *}$, M. Oms-Arias ${ }^{2}$, P. Modamio' ${ }^{1}$, A. Pedraza-Gutiérrez ${ }^{2}$, C. F. Lastra ${ }^{1}$, E. L. Mariño ${ }^{1}$

${ }^{1}$ Clinical Pharmacy and Pharmacotherapy Unit. Department of Pharmacy and Pharmaceutical Technology, and Physical Chemistry, Faculty of Pharmacy and Food Sciences, University of Barcelona, Barcelona, ${ }^{2}$ Costa de Ponent Primary Care Directorate, Catalan Institute of Health, L'Hospitalet de Llobregat, Barcelona, Spain

Background and objective: Opioid analgesics such as fentanyl are prescribed for moderate to severe pain. Fentanyl transdermal patches are also available as an alternative to strong oral opioids in cancerrelated pain as well as for persistent and intense pain in chronic nonmalignant conditions. The aim of this study was to describe the use of fentanyl patches in the treatment of pain in day-today practice and the subsequent pharmaceutical intervention if necessary.

Setting and method: A cross-sectional descriptive and multicenter study was performed. Setting: 53 primary health care centres covering 1.250.000 inhabitants. Target population: Patients with an active prescription of fentanyl transdermal patches on March 28, 2018. The Summary of Product Characteristics (SmPC) of all marketed (brandnamed and generic) medicines containing patches forms of fentanyl were reviewed to evaluate how these medicines were used.

Main outcome measures: Doses, dosage intervals, and appropriateness in the pattern of use of fentanyl patches according to the SmPC. Results: There were 4218 patients with an active prescription of fentanyl patches on March 28, 2018. 4.45\% $(\mathrm{N}=188)$ with prescriptions of divided patches $(0.5,0.75,1.2,1.25,1.5,2.5)$, and $3.08 \%$ $(\mathrm{N}=130)$ with a prescription with a dosage interval different from $72 \mathrm{~h}$. Some important information obtained from the different SmPC reviewed were the following: "The patch should not be cut, since 
there is no information available on the quality, efficacy and safety of the divided patch"; and "more than one patch can be used to achieve the desired dose". Besides, the patches have a systemic action and a continuous absorption through the skin for $72 \mathrm{~h}$. The $2.5 \mathrm{mg}, 5 \mathrm{mg}$ and $10 \mathrm{mg}$ patches liberate $25 \mathrm{mcg} / \mathrm{h}, 50 \mathrm{mcg} / \mathrm{h}$ and $100 \mathrm{mcg} / \mathrm{h}$ respectively. Therefore in case this pattern is modified, for instance, the patch is removed before $72 \mathrm{~h}$, then the patch would be a waste since it could still release fentanyl or if the patch is adhered to the skin for a period of time longer than $72 \mathrm{~h}$, then the patch would not be effective anymore since there would be no more fentanyl left to release. Therefore, it is highly advisable to readjust the prescriptions for those patients with divided patches and those with a dosage interval different from $72 \mathrm{~h}$.

Conclusion: $7.53 \%$ of the treatments analysed in this study had an inappropriate prescription. Pharmacist interventions addressed to the physicians were performed to promote a safer and more effective use of medicines.

Disclosure of Interest: None Declared.

PE002: Hormonal contraceptives use in Norway 2004-2016: a nationwide register-based study

\section{E. Aares ${ }^{1,2, *}$, Ø. Karlstad ${ }^{1}$, K. Furu ${ }^{1}$}

${ }^{1}$ Norwegian Institute of Public Health, ${ }^{2}$ School of Pharmacy, University of Oslo, Oslo, Norway

Background and objective: Since 2002, public health nurses and midwifes have had the right to prescribe oral contraceptives for women 16-19 years. This arrangement has been changed several times and from 2016 gave them right to prescribe all contraceptives including long acting reversible contraception (LARC) to all women over 16 years. In the last years, LARC have been increasingly recommended in most countries.

The objectives were to examine how hormonal contraceptive use in Norway has developed during 2004-2016, including choice of contraceptive method (oral contraceptives or long-acting reversible contraceptives (LARC)). In addition, we wanted to study what profession prescribes the different contraceptives.

Setting and method: Data on prescribed hormonal contraceptives dispensed to women was provided by the nationwide Norwegian Prescription Database. All women in Norway who had been dispensed contraceptives at least once during 2004-2016 ( $\mathrm{N}=987,889)$ were included.

Main outcome measures: Hormonal contraceptives (Anatomical Therapeutic Chemical codes G03A, G02B and G03HB) and profession of the prescriber.

Results: In 2016, 393978 (34\% of the population) women 16-49 years of age received hormonal contraceptives at least once. The annual prevalence of use was stable during 2004-2016, ranging from 31 to $34 \%$. The most commonly used contraceptive was combined oral contraception pills, $20 \%$ in 2016. Women 20-24 years old had the highest prevalence, ranging from 60 to 64\%. From 2014 to 2016 the use of LARC increased, especially in younger women: the prevalence increased from 2.8 to $8.6 \%$ among 16-19 year olds. $5.0 \%$ and $4.1 \%$ of women aged 16-19 years were prescribed LARC by doctors in 2015 and 2016, respectively. In 2016, 3.4\% of these young women were prescribed LARC by public health nurses. The most used LARC for 16-24 years was subdermal implants, while for women over 25 years it was intrauterine devices.

Conclusion: The overall use of hormonal contraceptives was stable during 2004-2016. Use of LARC increased especially in younger women. Public health nurses and midwifes contributed largely to the increase in use of LARC among young women in 2016. Disclosure of Interest: None Declared.
PE003: Drug burden measures and physical function in older adults with intellectual disabilities

J. O'Connell, 1,2,", E. Burke², M. O'Dwyer ${ }^{1}$, P. McCallion ${ }^{3}$, M. McCarron ${ }^{4}$, M. C. Henman ${ }^{1}$

${ }^{1}$ School of Pharmacy \& Pharmaceutical Sciences, ${ }^{2}$ IDS-TILDA, School of Nursing and Midwifery, Trinity College Dublin, Dublin, Ireland, ${ }^{3}$ College of Public Health, Temple University, Philadelphia, United States, ${ }^{4}$ Dean of Health Sciences, Trinity College Dublin, Dublin, Ireland

Background and objective: The Anticholinergic Cognitive Burden (ACB) scale and Sedative Load (SL) model are two measures of drug burden. Older adults with intellectual disabilities (ID) report high exposure to anticholinergic and sedative medications. Exposure to these medications has been associated with poorer physical function in older adults without ID.

Objective: To evaluate the relationship of ACB scores and SL scores with two physical function measures, grip strength and timed up and go (TUG) in a cohort of older adults with ID.

Setting and method: Wave 2 data from the Intellectual Disability Supplement to the Irish Longitudinal Study on Ageing (IDS-TILDA), a nationally representative study of older adults with ID in Ireland, was evaluated. Analysis of Covariance (ANCOVA) was used to detect associations with grip strength and TUG. In order to produce a robust analysis, continuous grip strength scores were separated by gender and analysed individually for males and females because of the inherent differences in grip strength depending on gender. Timed up and go scores were reciprocal transformed in order to achieve a robust ANCOVA across all groups.

Main outcome measures: Both drug burden measures were tested as categorical variables. ACB scores of $0,1-4, \geq 5$ and SL scores 0 , $1-2, \geq 3$ corresponded to no, low and high exposure respectively. These variables were used to measure associations with grip strength separated by gender $(\mathrm{kg})$ and reciprocal transformed TUG scores (1/ seconds).

Results: Grip strength was not significantly associated with ACB or SL score for either gender (males: ACB $p=0.618$, SL $p=0.818$; females: $\mathrm{ACB} p=0.850$, SL $p=0.952$ ) after adjusting for confounders. TUG was not significantly associated with ACB or SL $(p=0.066$ and $p=0.869)$.

Conclusion: The ANCOVA model produced a more robust analysis of associations between drug burden and physical function measures than previously used. There was no significant association between exposure to anticholinergic or sedative medications and physical function. These findings are similar to previous research in this cohort on associations of Drug Burden Index with physical function.

Disclosure of Interest: None Declared.

CP-PC013: Willingness of patients to use unused medications returned to the pharmacy by another patient

C. Bekker ${ }^{1, *}$, B. J. van den Bemt ${ }^{1}$, A. C. Egberts ${ }^{2}$, M. L. Bouvy ${ }^{3}$, H. Gardarsdottir ${ }^{2}$

${ }^{1}$ Department of Pharmacy, Sint Maartenskliniek, Nijmegen, ${ }^{2}$ Department of Clinical Pharmacy, Division Laboratory and Pharmacy, Utrecht University Medical Centre, ${ }^{3}$ Division of Pharmacoepidemiology and Clinical Pharmacology, Utrecht Institute for Pharmaceutical Sciences, Utrecht University, Utrecht, Netherlands

Background and objective: Redispensing by pharmacies of medications unused by another patient could contribute to optimal use of healthcare resources. Patient co-operation is essential for implementation of the redispensing process. However, insight into patient 
willingness to use redispensed medication is limited. This study aims to assess patient willingness to use medication returned by another patient and patient characteristics associated with this willingness.

Setting and method: A survey was conducted in 41 community and 5 outpatient pharmacies between April-December 2014 in the Netherlands. Pharmacy visitors completed a questionnaire regarding their willingness to use medication returned unused to the pharmacy by another patient, assuming quality was guaranteed. Patient sociodemographic characteristics were also collected and their associations with patient willingness were analysed using logistic regression analysis, reported as odds ratios (OR) with $95 \%$ confidence intervals. Main outcome measures: The proportion of patients willing to use redispensed medication and patient characteristics associated with this willingness.

Results: In total, 2215 patients (mean [SD] age 50.6 [18.0] years; $61.4 \%$ female) participated, $6.4 \%$ were returning medication for disposal. The majority $(61.2 \%)$ was willing to use medication returned unused to the pharmacy by another patient. Patients who were unwilling mostly found it too risky. Men were more willing to use returned medication (OR 1.3 [1.1-1.6]), as did patients with a high educational level (OR 1.8 [1.3-2.5]), those who regularly use 1-3 medications (OR 1.3 [1.1-1.7]), those who returned medication to the pharmacy for disposal (OR 1.5 [1.0-2.3]) and those who ever had unused medications themselves (OR 1.3 [1.1-1.6]). Patients with nonDutch cultural background were less willing to use returned medication (OR 0.3 [0.3-0.4]).

Conclusion: When quality is guaranteed, a substantial proportion of patients are willing to use medication returned unused to the pharmacy by another patient. This suggests that implementation of redispensing is likely to be supported by a majority of patients. Further elucidating and addressing reasons for patient unwillingness may increase the success rate of implementation.

Disclosure of Interest: None Declared.

PH002: Patients' perspectives about the tuberculosis control programme in Recife, Brazil: a contribution to evaluation

S. S. Bezerra ${ }^{1}$, M. P. Guerreiro ${ }^{2,3, *}$, M. P. G. Athayde ${ }^{4}$, J. J. F. Gomes ${ }^{5}$, J. L. S. Sobrinho ${ }^{6}$

${ }^{1}$ Post Graduation Program in Therapeutic Innovation, Federal University Of Pernambuco, Recife, Pernambuco, Brazil, ${ }^{2}$ Unidade de Investigação \& Desenvolvimento em Enfermagem, Escola Superior de Enfermagem de Lisboa, Lisboa, ${ }^{3}$ Centro de Investigação

Interdisciplinar, Instituto Superior de Ciências da Saúde Egas Moniz, Monte de Caparica, Portugal, ${ }^{4}$ Pneumology, Otávio de Freitas Hospital, Recife, Pernambuco, Brazil, ${ }^{5}$ Faculdade de Ciências, Universidade de Lisboa, Lisboa, Portugal, ${ }^{6}$ Pharmaceutical Sciences, Federal University Of Pernambuco, Recife, Pernambuco, Brazil

Background and objective: Evaluation is integral to health programmes and the perspective of users has long been regarded as one of its component. This study aims to contribute to evaluation of the tuberculosis control programme (PCT) in Recife, by describing patients' perspectives about this programme.

Setting and method: The study took place between July 2014 and February 2015 in five health units in Recife: two basic health units, one polyclinic, a university hospital of medium complexity and a hospital of high complexity. All eligible PCT outpatients in these sites were invited to participate $(\mathrm{n}=123)$. The data collection instrument, administered by trained interviewers, included questions on socioeconomic variables and 16 closed questions, derived from previous studies. Questions were grouped into three categories: organizational, economical and geographical accessibility. Answers were collected on a five-point scale, ranging from 1 to 5 , and converted into scores.
Statistical analysis was performed using R software. Ethical approval was granted.

Main outcome measures: Total score per patient. Mean score per category.

Results: Forty-four patients agreed to be interviewed. The majority were male (77\%); age ranged between 23 and 65 years (median 45.6). Most had a family income of less than four minimum wages or no income (97\%) and declared being non-smokers (90\%). Overall patients' perspectives regarding the PCT were unfavourable (median score $35 \%$; third quartile $46.5 \%$ ). Differences between median scores in the sites were not statistically significant (Kruskal-Wallis test; $p=0.393$ ). Less positive scores were attributed to home visits by health professionals and receiving information on $\mathrm{TB}$ and its treatment. The most positive score pertained to access to TB medication. Analysis of scores by categories showed statistically significant differences (Wilcoxon signed rank test, $\mathrm{V}=715.5, p$ value $=0.005$; Paired $t$ test, $\mathrm{t}=2.92$, $\mathrm{df}=43, p$ value $=0.003$ ). Organisational accessibility received the least favourable opinion.

Conclusion: Patients' perspectives on the PCT highlighted potential opportunities for improvement in the sites surveyed. Findings can be used as a starting point to a more in-depth analysis on the use of resources, particularly in respect to organisational acceptability. Several aspects of organisational acceptability, such as providing information about TB and its treatment, may be improved by resorting to pharmacists dispensing TB medication.

Disclosure of Interest: None Declared.

\section{Poster discussion forum II: Hospital pharmacy}

HP-PC010: Evaluation of the use of dabigatran etexilate in patients with atrial fibrillation treated with an electrical cardioversion

P. Nieto Gómez ${ }^{1}$, R. Álvarez Sánchez ${ }^{1, *}$, A. Rodríguez Delgado ${ }^{1}$, P. Moreno Raya ${ }^{1}$, I. Casas Hidalgo ${ }^{1}$, M. Valle Corpas ${ }^{1}$

${ }^{1}$ Farmacia Hospitalaria, Hospital Clinico San Cecilio, Granada, Spain

Background and objective: The objective of this study is to describe the use of dabigatran etexilate in patients with atrial fibrillation who undergo an electrical cardioversion, indication that is currently an offlabel use in our country.

Setting and method: A search was made in the clinical records of 35 patients who used dabigatran etexilate as an oral anticoagulant agent as prophylaxis of thrombotic events in electrical cardioversion during 2017.

Main outcome measures: Demographic data (age, sex), number of days anticoagulated, hemorrhagic risk factors, and thrombotic risk factors were collected. With these data, the thrombotic risk (CHA2DS2-VASC) and the haemorrhagic risk (HAS-BLED) were calculated.

Results: All patients were anticoagulated with dabigatran etexilate $150 \mathrm{mg}$ twice daily. The median age of the patients was 58 (49.5-64.5). 88.57\% were men. The median of days of anticoagulation was 90 (60-190.5). 65.71\% of the patients had CHA2DS2-VASC less than 2 and $34.29 \%$ had a risk of 0 . Regarding the haemorrhagic risk, $31.42 \%$ of the patients had a HAS-BLED greater than 2. Among patients with a low risk CHA2DS2-VASC (less than 2 (men) and less than 3 (women)), the median number of days of anticoagulation after the intervention was 69 (39-210). 60\% of these patients were more than 30 days anticoagulated after the intervention and $38 \%$ continued until the day that the data were collected. Patients with a CHA2DS2VASC risk of 0 were 69 days (60.5-374.5) anticoagulated after the intervention. In addition, 2 of the 3 patients who had a HAS-BLED 
greater than CHA2DS2-VASC were anticoagulated for more than a year, with a CHA2DS2-VASC of 1.

Conclusion: According to the guidelines, the decision of maintaining anticoagulation in patients with low thrombotic risk has to be made based on the haemorrhagic and thrombotic risk of these and it should be maintained for at least 4 weeks after the intervention, without needing more time in patients with CHA2DS2-VASC risk 0. In this study we observed that patients with low thrombotic risk remained anticoagulated more than 4 weeks, being especially relevant patients with a CHA2DS2-VASC of 0 who were more than a year anticoagulated with dabigatran etexilate and exposed to potentially serious adverse events.

Disclosure of Interest: None Declared.

\section{HP-PC011: Evaluation of pharmacist clinical interventions} and discharge counselling in medical rehabilitation wards

\section{N. S. R. Ip ${ }^{1, *}$, C. K. A. Chu ${ }^{2}$, L. M. P. Chu ${ }^{1}$, W. M. G. Young ${ }^{1}$,} J. W. Tenney ${ }^{3}$

${ }^{1}$ Pharmacy, ${ }^{2}$ Department of Medicine and Geriatrics, Tuen Mun Hospital, ${ }^{3}$ School of Pharmacy, The Chinese University of Hong Kong, Hong Kong, Hong Kong

Background and objective: Drug related problems (DRPs) are common occurrence in hospitalized patients. Previous literature showed clinically important medication errors affected over $50 \%$ of patients during the first 30 days after hospital discharge. Patients with cardiovascular/cerebrovascular disease, especially heart failure, acute coronary syndrome and stroke are particularly prone to DRPs due to new and complex medication regimen prescribed after discharge. This study aims to evaluate the prevalence of clinically significant DRPs, the effect of pharmacist intervention on post-discharge unplanned healthcare utilization and discharge counselling on medication adherence in medical rehabilitation wards.

Design: Prospective, quasi-experimental study conducted in 4 medical rehabilitation wards in a local hospital. The prospective intervention group was compared to a historical standard care group. Clinical pharmacist screened eligible patients for enrolment then provided medication reconciliation and discharge counselling. Patients were phoned 30-days after discharge to assess for re-admission rate and medication adherence by using the 8-item Morisky instrument.

Results: A total of 85 patients $(n=43$ in prospective intervention group, $\mathrm{n}=42$ in historical control group) were included in the study. Among the intervention group, 23 DRPs were identified in $14(32.6 \%)$ of patients, resulting in 51 interventions. The pharmacist intervention group had a lower rate of unplanned healthcare utilization $25.6 \%$ (11 of 43) compared to the control group 47.6\% (20 of 42) $(p=0.035)$. The proportion of patients reporting medium or high medication adherence by having a Morisky score of $\geq 6$ increased from $23.6 \%$ pre-counselling to $88.4 \% 30$ days after counselling $(p<0.05)$.

Conclusion: Pharmacist medication reconciliation and discharge counselling significantly reduced unplanned healthcare utilization in the next 30 days post-discharge and improved patient medication adherence.

Disclosure of Interest: None Declared.

HP-PC012: Benefit of a clinical pharmacist on a German surgical intensive care unit: a controlled interventional study

N. Kessemeier ${ }^{1, *}$, D. Meyn ${ }^{1}$, M. Höckel ${ }^{1}$, J. Reitze ${ }^{2}$, C. Culmsee ${ }^{3}$, M. Tryba $^{4}$

${ }^{1}$ Hospital Pharmacy, Gesundheit Nordhessen Holding AG, Kassel,

${ }^{2}$ Statistics, MoreDATA, Giessen, ${ }^{3}$ Pharmacology and Clinical
Pharmacy, Philipps-University Marburg, Marburg, ${ }^{4}$ Kassel School of Medicine, Gesundheit Nordhessen Holding AG, Kassel, Germany

Background and objective: Previous international studies suggested beneficial effects of a clinical pharmacist's participation on an intensive care unit (ICU) on a variety of relevant parameters such as reduction of medication errors, drug costs and improvements of clinical outcomes such as ICU length of stay. Clinical pharmacist's influence on these outcomes on German ICUs has not been investigated so far. International findings are not transferable to the German healthcare system and establishing clinical pharmacy services on the ward is not well-advanced in Germany. This controlled interventional study was designed to investigate a clinical pharmacists' impact on medication errors on a German surgical ICU.

Setting and method: The study was divided in three phases. One control phase $\mathrm{P}_{0}$ and two intervention phases $\mathrm{P}_{1}$ and $\mathrm{P}_{2}$. Throughout the control phase $P_{0}$ the data were collected without the clinical pharmacists' intervention. In the following intervention periods $P_{1}$ and $\mathrm{P}_{2}$, two clinical pharmacists screened the medical records for drug related problems and discussed them with the senior physician. During $\mathrm{P}_{2}$ clinical pharmacists attended ward rounds additionally. Drug related problems detected by the clinical pharmacists according to defined criteria, were taken into account as medication error if a consensus with the physician was achieved.

Main outcome measures: The investigation aimed to determine if the medication review by a clinical pharmacist results in a significant reduction of medication errors related to a control period. Secondary outcomes were the reduction of potentially severe medication errors, the number of days without systemic anti-infective therapy the length of stay.

Results: The medication orders of 336 patients were reviewed. The number of medication errors was significantly reduced from 1660 to 622 respectively $401\left(\mathrm{P}_{0}\right.$ vs. $\mathrm{P}_{1} / \mathrm{P}_{2}$; both $P<0.05$; Fisher's Exact Test) in total, respective $14.1 \%$ in $\mathrm{P}_{0}$ vs. $5.1 \%$ in $\mathrm{P}_{1}$ respectively $3.3 \%$ in $\mathrm{P}_{2}$ related to the monitored medications $\left(\mathrm{P}_{0} 11,755 ; \mathrm{P}_{1} 12,134 ; \mathrm{P}_{2}\right.$ 12,329).

Conclusion: Clinical pharmacists' interventions led to a significant reduction of medication errors in a German ICU, contributing to a safer medication process. We strongly recommend a broad implementation of clinical pharmacists on German ICUs.

Disclosure of Interest: None Declared.

HP-PC013: Drug related hospital admissions: identifying highrisk patients

L. D. Nymoen ${ }^{1,2, *}$, I. K. Solsvik ${ }^{1}$, T. E. Flateb ${ }^{2}$, M. Nilsen ${ }^{2}$, A. Godø ${ }^{3}$, E. Øie ${ }^{4}$, K. K. Viktil ${ }^{1,2}$

${ }^{1}$ School of Pharmacy, University of Oslo, ${ }^{2}$ Diakonhjemmet Hospital Pharmacy, ${ }^{3}$ Department of Anaesthesia and Intensive Care,

${ }^{4}$ Department of Internal Medicine, Diakonhjemmet Hospital, Oslo, Norway

Background and objective: Drug related hospital admissions (DRHA) are a recognized challenge affecting patient safety worldwide. However, DRHA can easily be overlooked due to the fast-paced work-flow in the emergency department, and insufficient information about the patient's actual use of medication. Several studies claim that a significant proportion of these admissions could be prevented. Despite this, the incidence of DRHA are scarcely investigated in Norway. Additionally, risk factors for DRHA are not well studied. In this study we aimed to investigate incidence, characteristics as well as identifying risk factors for DRHA.

Setting and method: This study was part of a randomised controlled trial; patients were included and randomised at arrival to the emergency department, Diakonhjemmet Hospital. The intervention group: 
Medication reconciliation and medication review were conducted by a clinical pharmacist at admission. Relevant patient characteristics were registered and drug-related problems were identified. The control group got standard care, i.e. without pharmacist involved. DRHA were investigated retrospective in the intervention group patients by a multidisciplinary team (clinical pharmacists and senior physicians). The members of the multidisciplinary team evaluated each hospital admission, first individually, then together in a consensus meeting. Binary logistic regression was used to determine risk factors.

Main outcome measures: incidence of DRHA, characteristics of these admissions and risk factors correlated with the occurrence of these admissions.

Results: 180 patients were included, 5\% and $14.4 \%$ of these were assessed to have a probable or a possible DRHA, respectively. The most frequent drug-related problem leading to DRHA was adverse drug reactions $(51.4 \%)$. In addition, it was revealed that interactions $(17.1 \%)$ and treatment failure $(17.1 \%)$ frequently led to DRHA. We also revealed that drug use deviating from the prescription caused DRHA (14.3\%). Drugs frequently involved in DRHA were drugs affecting the blood pressure, antithrombotic drugs, Non-steroidal Anti-Inflammatory Drugs, and drugs against obstructive pulmonary disease. Identified risk factors: Women had a 2.7 times higher odds ratio of DRHA than men, and the odds ratio of DRHA increased with $3.7 \%$ per year added to the age. $77 \%$ of the DRHA were linked to drug-related problems discovered by the clinical pharmacists in the emergency department.

Conclusion: In this study, we have revealed that one in five patients had DRHA. Our study indicates that conducting medication reconciliation and medication review in the emergency department can reveal DRHA at an early point of the hospital admission. Further, the revealed risk factors (risky drugs, gender and age) can be used to identify high-risk patients and contribute to personalise the health care and hopefully prevent DRHA.

Disclosure of Interest: None Declared.

HP-PC014: In the era of cancer immunotherapy: time for pharmacist to redefine the prescription validation of immune checkpoint inhibitors

\section{J. Clarenne ${ }^{1, *}$, F. Slimano ${ }^{1,2}$, C. Boulanger ${ }^{1}$, A. Maréchal ${ }^{1}$, D. Hettler ${ }^{1}$}

${ }^{1}$ Department of Pharmacy, CHU Reims, ${ }^{2}$ Faculty of Pharmacy, Reims University, Reims, France

Background and objective: Immune Checkpoint Inhibitors (ICI) targeting CTLA-4 (Ipilimumab) and the PD-1/PD-L1 axis (Nivolumab and Pembrolizumab) has dramatically improved the treatment of several cancer such as malignant melanoma (MM) or non-small cell lung cancer (NSCLC). Moreover, ICI don't expose patients to classic adverse events (AE) like haematological toxicity. However, and based on their ability to reinvigorate immune system against tumour, ICI can expose to immune-related Adverse Events (irAEs). Treatment discontinuation occurs when irAEs grade 3-4 and can lead to permanent discontinuation. Pharmacist validation of medical order can ordinarily detect and prevent AE using biological data like blood count but in the era of ICI it seems necessary to redefine an appropriate checklist linked to irAEs in order to improve medication safety with ICI.

Setting and method: Retrospective study in a university hospital including all patients who received at least one ICI treatment between 1st October 2011 and 31 December, 2017. All treatment discontinuation ( $\geq 7$ days from the planned date) were collected from electronic health records (CHIMIO ${ }^{\circledR} 5.7$, Millenium ${ }^{\circledR}$, Easily ${ }^{\circledR}$ ). The reasons of discontinuation were classified as toxic (linked to irAEs) or other and whether temporary or permanent. Results were compared to literature and a new checklist for pharmacist validation was proposed to four clinical pharmacists.

Main outcome measures: The primary endpoint was to assess prevalence of irAEs G3-4 leading to temporary or permanent treatment discontinuation. Secondary endpoint was to build a new checklist for pharmacists' validation dedicated to ICI prescription.

Results: A total of 238 patients were included, with $\mathrm{n}=135$ male $(57 \%)$ and mean age 63 years [28-93]. Patients received ICI for MM $(\mathrm{n}=193 ; 81.1 \%)$, NSCLC $(\mathrm{n}=44 ; 18.5 \%)$ and Hodgkin lymphoma $(\mathrm{n}=1 ; 0.4 \%)$. The occurrence of G3-4 irAEs for each drug was $\mathrm{n}=27(42.9 \%)$ for Nivolumab, $\mathrm{n}=26(26 \%)$ for Pembrolizumab and $\mathrm{n}=10(14.1 \%)$ for Ipilimumab, respectively. Main G3-4 irAEs were diarrhoea and colitis $(n=23 ; 9.8 \%)$, pneumonitis $(n=10 ; 4.3 \%)$ and hepatitis $(n=4 ; 1.7 \%)$ and these data were consistent with literature. Global G3-4 irAEs lead to $n=29(12.2 \%)$ temporary and $n=33$ (13.9\%) definitive treatment discontinuation, respectively.

Conclusion: Our study confirms the safety profile of ICI with the occurrence of irAEs instead of classic AE. Pharmacist validation should take into account of new biological data as thyroid-stimulating hormone, prolactin and liver enzymes. Rare acute renal injury cases with ICI were recorded in our study as well as in literature and it does not support the abandonment of creatininemia in the new checklist. However, blood count seems to be to useless to prevent irAEs. These new proposition were discussed and accepted by the four clinical pharmacists and further studies will be warranted to asses this new checklist in clinical practice.

Disclosure of Interest: None Declared.

HP-PC015: Effectiveness of the Ganzoni Formula for calculating ferric-carboxymaltose dose in patients with ferropenic anemia

N. Martín-Gutierrez ${ }^{1, *}$, D. Peña-Lorenzo ${ }^{1}$, N. Rebollo-Díaz ${ }^{1}$, J. G. Sanchez-Hernández ${ }^{1}$, M. Martín-Gil ${ }^{1}$, R. Fernández-Caballero ${ }^{1}$, R. Martín-Muñoz ${ }^{1}$, M. J. Otero-López ${ }^{1}$

${ }^{1}$ Pharmacy, Complejo Asistencial Universitario De Salamanca, Salamanca, Spain

Background and objective: Ferric-carboxymaltose is an iron complex, controlling iron delivery to tissues. At our hospital, dose is calculated using the Ganzoni Formula to reach a target haemoglobin level $(\mathrm{Hb})$ of $13 \mathrm{~g} / \mathrm{dL}$ for men and $12 \mathrm{~g} / \mathrm{dL}$ for women. However, the Summary of Product Characteristics (SPC) proposes a table based on body weight and $\mathrm{Hb}$.

A previous analysis at our centre comparing both dosage regimens concluded that doses estimated by the Ganzoni Formula were significantly lower than those proposed in the SPC, so we decided to test its effectiveness.

The objective was to evaluate the effectiveness of the Ganzoni Formula for establishing an optimal dose to reach the target $\mathrm{Hb}$ for ferric-carboxymaltose in patients with ferropenic anemia.

Design: A retrospective study conducted at a 1100-bed hospital in patients who received ferric-carboxymaltose over a 3-month period. Our source of information for ferric-carboxymaltose prescription was the standardized document.

The following variables were collected: dose, sex, age, weight, $\mathrm{Hb}$, ferritin and transferrin saturation (TSAT). Data from the blood test obtained 4 weeks after final iron administration were evaluated, and the need for additional doses was recorded.

Comparison of $\mathrm{Hb}$ values, determined before and after ferriccarboxymaltose administration, was performed with the $\mathrm{R}^{\circledR}$ software. Since data were not normally distributed, the Wilcoxon test was applied.

Results: 46 patients (17 men) were included with a median [rank] age and weight of 64[42-90] years and 83[35-95] kg, respectively. Before 
treatment, values for $\mathrm{Hb}$, ferritin and TSAT were: 9.45[6.6-14.9] $\mathrm{g} / \mathrm{dL}, 24[5-296] \mathrm{ng} / \mathrm{mL}$ and 6.25[2.1-39] \%.

Patients received 900[500-2000] $\mathrm{mg}$ of ferric-carboxymaltose, according to the Ganzoni Formula. New laboratory tests were performed 49.5[26-187] days after the last iron administration, and values for $\mathrm{Hb}$, ferritin and TSAT were: 11.2[6.2-15.4] $\mathrm{g} / \mathrm{dL}$, 111 [10-1049] $\mathrm{ng} / \mathrm{mL}$ and 18.45 [2.37-299] respectively.

Although $\mathrm{Hb}$ was significantly higher after iron administration $(p<0.001)$, only 16 patients achieved the target, and 15 required additional doses.

Conclusion: Although analytical value improvement was observed using the Ganzoni Formula for ferric-carboxymaltose dose calculation, the $\mathrm{Hb}$ target was not reached in most patients. Since this formula is proposed in several guidelines, future studies comparing it with the regimen proposed in the SPC would be desirable.

Disclosure of Interest: None Declared.

\section{HP-PC016: Stratification of patients for drug related problem-} reduction service, using predictive modeling and high alert medications approaches

\section{G. Al-Taani ${ }^{1 \text {,* }}$, S. Al-Azzam ${ }^{2}$, K. Alzoubi ${ }^{3}$, F. Darwish Elhajji ${ }^{4}$,

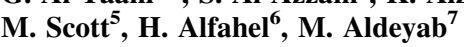

${ }^{1}$ Faculty of Pharmacy, Yarmouk University, ${ }^{2}$ Jordan University of Science and Technololgy, ${ }^{3}$ Faculty of Pharmacy, Jordan University of Science and Technology, Irbid, ${ }^{4}$ Faculty of Pharmacy, Applied Science Private University, Amman, Jordan, ${ }^{5}$ Medicines Optimisation Innovation Centre, Northern Health and Social Care Trust, Northern Ireland, United Kingdom, ${ }^{6}$ Faculty of Medicine, Hashemite University, Zarqa, Jordan, ${ }^{7}$ School of Pharmacy and Pharmaceutical Science, University of Ulster, Coleraine, United Kingdom

Background and objective: Drug-related problems (DRPs) are considered care issues commonly addressed by pharmacist. Case finding is continuous requirements for DRP-reduction service. Predictive modelling has been utilized as robust approach for case finding, using statistical adjustment. The present presentation provides an insight about predictive modelling, describe a predictive model developed for important DRPs and the utility of high alert medications approach for case finding.

Setting and method: I. Patients with diabetes from outpatient clinics from five key university-affiliated and public hospitals in Jordan were assessed for DRPs (drug without an indication, untreated indication, and drug efficacy problems). Potential risk factors for DRPs were assessed. A logistic regression model was used to identify risk factors using a randomly selected, independent, non-overlapping development $(75 \%)$ subsample from full dataset. The remaining validation subsample $(25 \%)$ was reserved to assess the discriminative ability of the model

II. DRPs were assessed for patients recruited from outpatient clinics in five major hospitals in Jordan using a standardized and validated pharmaceutical care manual.

Main outcome measures: Risk factors for DRPs and drugs associated with different types of DRPs.

Results: I. A total of 1494 patients were recruited. Of them, 81.2\% had at least one DRP. Using the development subsample $(n=1085)$, independent risk factors for DRPs identified were male gender, number of medications, prescribed gastrointestinal medication, and nonadherence to self-care and non-pharmacological recommendations. Validation results $(n=403)$ showed an area under the receiver operating characteristic curve of 0.679 (95\% confidence interval $=0.629-0.720$ ); the model sensitivity and specificity values were $65.4 \%$ and $63.0 \%$, respectively.

II. Ultimately, 2747 patients, with a total of 10,672 DRPs, were included in the study. The medication groups most commonly associated with
DRPs were cardiovascular (53.0\%), endocrine (18.1\%), and gastrointestinal $(7.7 \%)$ drugs. The most common specific drugs associated with DRPs from any category were atorvastatin $(12.5 \%)$, metformin $(8.5 \%)$, simvastatin $(6.2 \%)$, and enalapril (5.9\%). Cardiovascular medications were the most common drugs implicated with many subtypes of DRPs-most commonly, allergic reaction or undesirable effect $(88.5 \%)$, drug product not available $(87.3 \%)$, safety interaction issues $(81.8 \%)$, a need for additional or more frequent monitoring $(78.0 \%)$, and more effective drugs are available (77.2\%). Hypertension, diabetes mellitus, and dyslipidemia were the most common diseases associated with different subtypes of DRPs.

Conclusion: The present study addressed multiple targeting approaches that can be used clinically for case findings to address DRPs, that were assessed comprehensively.

Disclosure of Interest: None Declared.

HP-PC017: LACE tool: enables appropriate patient prioritisation for elderly patients at discharge?

E. Clark ${ }^{1, *}$, P. R. Mills ${ }^{1}$

${ }^{1}$ Pharmacy, University Hospital Crosshouse, Kilmarnock, United Kingdom

Background and objective: An ageing population places increased demand on healthcare. Hospital pharmacy staff resources are limited and patient numbers exceed pharmaceutical care capacity. The LACE tool developed in Canada to identify elderly patients for enhanced discharge care. However, one United Kingdom trial indicated poor correlation with patient need. The aim was to evaluate the effectiveness of the LACE tool in identifying patients who require enhanced pharmaceutical care at discharge.

Setting and method: Four care of the elderly wards across one United Kingdom district general hospital and one community hospital. LACE score calculated for patients medically fit for discharge. LACE score calculated according to length of stay, acuity, co-morbidity and emergency department visits. Patients scoring $\geq 10$ (high risk of re-admission or death) interviewed to complete a medicines management assessment. Assessed in form of questionnaire posed to patients directly by single assessor, to ascertain any medication related problems prior to admission. Data collated on Excel spreadsheet and analysed using descriptive statistics. Thematic analysis of verbal comments completed.

Main outcome measures: Correlation between LACE score and medicines management assessment.

Results: Study completed during a 4-week period ( $\mathrm{n}=28$ patients). $68 \%(\mathrm{n}=19)$ patients scored at high risk of re-admission or death (LACE score $\geq 10)$ with remaining $32 \%(n=9)$ deemed at low risk (LACE score $<10$ ). Patient length of stay was $>14$ days for 20 patients which was the main contributor for high risk patients. Average number of medicines was 9; median 9.5 (range 5-13).

A medicines management assessment was completed for $53 \%$ $(n=10)$ of high risk patients. All high-risk patients who completed assessment raised at least one medicines-related issue during interview. Key themes identified within this group $(n=10)$ included reliance on someone else to manage medicines, swallowing difficulties, cognitive impairment, and lack of knowledge of medicines. All patients would therefore benefit for enhanced pharmaceutical care at discharge in comparison to the traditional service of medicine supply with limited information provision.

Conclusion: Application of the LACE tool to an elderly patient group demonstrated correlation with patients' ability to manage medicines. High risk patients required enhanced pharmaceutical discharge care. Therefore, the LACE tool enables pharmacy resources to be targeted to appropriate patients.

Disclosure of Interest: None Declared. 
HP-PC018: The effect of VKORC1 gene polymorphism on warfarin maintenance dose in different ethnic pediatric patients

\section{P.-L. Hung ${ }^{1, *}$, A. C. Lin $^{2}$, P.-C. Lin $^{1}$}

${ }^{1}$ Pharmacy, Kaohsiung Veterans General Hospital, Kaohsiung, Taiwan, Province of China, ${ }^{2}$ College of Pharmacy, Cincinnati University, OHI, United States

Background and objective: Warfarin is a common anticoagulant with narrow therapeutic window and variable anticoagulation effects. Many studies have confirmed the relationship between warfarin maintenance doses and single nucleotide polymorphisms of cytochrome $\mathrm{P} 450$ 2C9 (CYP2C9), vitamin $\mathrm{K}$ epoxide reductase (VKORC1), and cytochrome P450 4F2 (CYP4F2) in adults, but their effects in children remain unclear. VKORC1 were responsible for the large inter-individual variability of the anticoagulant effect of warfarin but the role in paediatric patients remains controversial, especially in ethnic differences. We aimed to investigate the relationship between VKORC1 gene polymorphism and different warfarin dose in paediatric patients with Asian and non-Asian groups. Setting and method: A systemic review and meta-analysis were conducted. PubMed, EMBASE, and Cochrane library from 2004 to April 15, 2018 were searched.

Main outcome measures: The relationship between warfarin dose and two single nucleotide polymorphisms of the VKORC1 gene (at positions 1639 and 1173) were analysed using Revman version 5.3.5 software.

Results: Eleven studies combined total 867 paediatric patients were included in this meta-analysis. The frequency of the VKORC1 genetic polymorphisms was determined as wild type VKORC1-1639 GG (32.9\%), heterozygote alleles GA $(50.2 \%)$, and mutant alleles AA $(16.9 \%)$. Even in the different ethnicities, patients that were VKORC1-1639 GA, AA required significantly lower warfarin dosage than GG carriers to reach the therapeutic range. Compared to GG carriers, GA and AA carriers required $25 \%(95 \% \mathrm{CI}-0.28,-0.21)$ and $36 \%(95 \% \mathrm{CI}-0.56,-0.15)$ lower mean difference warfarin dose. On the other hand, VKORC1-1173 polymorphisms showed patients with a mutant homozygotic TT genotype required 62\% $(95 \%$ CI $0.22,1.02)$ lower mean difference warfarin dose than those with the heterozygotic TC genotype in Asia group, which is no significant different on no-Asia paediatric patients.

Conclusion: Previous studies have rarely been reported the influence of VKORC1 genotypes on warfarin dose requirements in different ethnic paediatric patients. Our result showed that polymorphisms in VKORC1 affected daily warfarin dosage requirements. VKORC1 1173 TT genotype is more strongly influenced warfarin dosage in Asia than non-Asia paediatric patients. Future studies shall utilize a large sample size to reconfirm our results.

Disclosure of Interest: None Declared.

HP-PC019: Patients are content with medicine information at hospital discharge-but do they get what they need?

E. Trapnes ${ }^{1,2, *}$, R. A. Hasan ${ }^{3}$, D. Nguyen', J. K. Sund ${ }^{3,4}$, L. Mathiesen ${ }^{2}$

${ }^{1}$ Hospital Pharmacies Enterprise, South Eastern Norway, ${ }^{2}$ Institute of Pharmacy, Oslo University, Oslo, ${ }^{3}$ Norwegian University of Science and Technology, NTNU, ${ }^{4}$ Central Norway Hospital Pharmacy Trust, Trondheim, Norway

Background and objective: Medicines reconciliation and medicines review are standardised tasks in in the Integrated Medicines Management (IMM)-model applied in Norwegian hospitals. Hospital discharge is a critical point of intervention for ensuring patients' continuity of care, but pharmacists' involvement is not yet decided. An understanding of patients' experiences and needs is warranted. Setting and method: 200 patients discharged from three medical wards at Oslo University Hospital were included and asked to fill in a questionnaire. Distribution of answers were calculated, and the Chi squared test was applied for comparisons between groups.

Furthermore, 12 patients were included for qualitative semi-structured interviews conducted within the first weeks after discharge. The interviews were recorded, transcribed, and analysed according to Malterud's method for systematic text condensation.

Main outcome measures: Patients' self-reported degree of satisfaction with medicines information at discharge, needs regarding medicine information and their drug use after discharge.

Results: The response rate to the questionnaire was $64 \%$ (126/200). A majority of the patients reported that they received information on discharge about their medicines. However, $23 \%$ of the patients claimed they did not receive any information. In general, patients expressed satisfaction with the information received, and women were significantly more satisfied than men. Patients were least satisfied with information on side effects and drug interactions. We observed incomplete understanding of the coherence between illness and prescribed drugs. The patients considered themselves responsible medicine users and organised their daily drug handling well. However, a majority of the patients reported receiving incomplete information concerning continuity of care following discharge. The patients expressed a desire to receive written and oral information.

Conclusion: Patients were mainly satisfied with the drug information received at discharge. However, a more profound drug information, including indication, effects, side effects and potential drug interactions are warranted by many patients. Written and oral information of further drug use must be ensured, and patients should be better informed about their continuity of care. Pharmacists have the required expertise to improve drug information given to patients at hospital discharge.

Disclosure of Interest: None Declared.

HP-PC020: Implementation of check of appropriateness (COA) in the back office validation of electronic prescriptions in az groeninge

\author{
J. Wittezaele ${ }^{1, *}$, S. Martens ${ }^{1}$, C. Vergote ${ }^{1}$, K. Verhelle ${ }^{1}$, \\ H. Collier ${ }^{2}$ \\ ${ }^{1} \mathrm{AZ}$ Groeninge, kortrijk, ${ }^{2} \mathrm{UZ}$ Brussel, Brussel, Belgium
}

Background and objective: Previous studies concluded pharmacists, within a multidisciplinary team, can influence the identification and prevention of medication errors. The aim of this study is to expand and standardize the clinical back office validation and implementation of the COA in the pharmacy back office (BO) activities of az groeninge in order to further reduce medication errors.

Setting and method: Prospective monocentric study; Before implementation of COA, a baseline measurement was executed. A second and third measurement were done after implementation, respectively the $\mathrm{BO}$ validation of ordered medication in 1st line (before dispensing) and validation of prescriptions generated in a query in 2nd line (after dispensing). Four queries were implemented in this research: posology of inhalers, IV/PO switch, overrules of very serious interactions and crushing medication. Tools for validation in BO 1st and 2nd line were made to improve the COA. Registration of the interventions was done by a pharmacist. These were recorded in an intervention form consisting of the following elements: the drug, intervention phase, reason, given advice, way of communication and acceptance degree.

Main outcome measures: The comparison of the results was made between the baseline measurement and BO 1st line, and the BO 1st line and the BO 2nd line (significant when $P$ values $<0.05$ ). 
Results: In this research a total of 18,558 electronic prescription lines were validated by a pharmacist of which 452 interventions were recorded. An error rate of $0.4 \%$ was intercepted during the baseline measurement, which increased significantly to a percentage of $1.1 \%$ during the BO 1st line. During the BO 2nd line, the error rate was even higher (7.2\%; significant) which shows that the implementation of the tools used in BO 1st and 2nd line ensure that even more prescribing errors can be intercepted. The acceptance rate was significantly lower in BO 2nd $(54.7 \%)$ compared to the BO 1st line $(81.0 \%)$. Which indicates that unidirectional communication of electronic notes, significantly more used in the BO 2nd line, isn't effective enough.

Conclusion: The COA has been successfully implemented which plays a role in the medication reconciliation of patients during hospitalization. Pharmacist interventions are important to intercept discrepancies of prescriptions at an early stage so the quality of care, drug therapy and patient safety is improved in az groeninge.

Disclosure of Interest: None Declared.

HP-PC021: Implementing clinical pharmacy practices in pediatric transplantation to improve patients' medication safety and adherence

\section{H. Capelle ${ }^{1, *}$, M. Peretti ${ }^{1}$, S. Atlan ${ }^{1}$, P. Bertault-Peres ${ }^{1}$, S. Honoré ${ }^{1}$}

${ }^{1}$ Pharmacy, Assistance Publique Hôpitaux de Marseille - Hôpital de la Timone, Marseille, France

Background and objective: More than half of the medication errors are related to transitions of care, and solid-organ transplant recipients are at an increased risk for errors due to significant changes in immunosuppressive medication (IS) following transplantation. The process of medication reconciliation is important especially in this population due to the risk for rejection and other complications in the post-transplant period. Our objective is to develop clinical pharmacy practices to improve paediatric transplant patients' medication safety, which can lead to improve clinical outcomes.

Setting and method: We conducted a prospective observational single-centre study from June 2015 to March 2018 in a paediatric solid-organ transplant population. Medication reconciliation and prospective data collection on an observational basis were performed. Low plasma IS levels was our indicator of non-adherence.

Main outcome measures: Hospital and community pharmacists have a central role in identifying and addressing the problem of non-adherence in children with chronic disease. Medication reconciliation upon admission associated with an interview with a clinical pharmacist at discharge can help to reduce drug related problems in paediatric solid-organ transplant patients.

Results: 60 patients were included (mean age $113 \pm 59$ months, sex ratio 1.2). Organs transplanted were kidney (40\%), liver (38\%), heart $(13 \%)$, liver + pancreas $(3 \%)$, lung $(2 \%)$, kidney + liver $(2 \%)$, kidney + heart $(2 \%)$. In medication history, we found $5 \pm 3$ medications per patient. 5 kind of immunosuppressive agents were prescribed (tacrolimus, mycophenolate mofetil, cyclosporine, azathioprine, everolimus). Monotherapy was mostly selected for liver transplants and combination therapy for kidney, heart and lung transplants. Medication reconciliation unveiled 13 unintentional discrepancies (UD), i.e. $0.2 \pm 0.8$ per patient. $10 \%$ of patients had at least 1 UD. The main identified UD were treatment omissions (69\%) and dosing errors $(25 \%)$, they were all corrected. 7 UD were related to IS and transplant rejection prophylaxis $(54 \%)$. 23 children presented with transplant rejection (38\%). Among them, we found low plasma IS levels in 8 children, associated with non-adherence, and 3 of them had at least $1 \mathrm{UD}$ identified with the medication reconciliation process.
Conclusion: Clinical pharmacist involvement in the transplant plan leads to improve safety through the reduction of medication errors and young patients' compliance to IS. In the future, our goal is to reinforce personalized pharmacy care by counselling patients and their family concerning their medication therapy and instructed them how to properly take their medication through a therapeutic education program for paediatric transplant recipients.

Disclosure of Interest: None Declared.

\section{Poster discussion forum III: Innovations in pharmaceutical care}

\section{HP-CE001: Effect of counselling on HbA1c and quality of life on T2DM patients}

\author{
A. Natasya $^{1}$, R. Andrajati ${ }^{1, *}$, R. Sauriasari ${ }^{1}$, W. N. Hening ${ }^{1}$ \\ ${ }^{1}$ University of Indonesia, Depok, Indonesia
}

Background and objective: Diabetes Mellitus (DM) patients requires adaptation of lifestyle and treatment to improve glycemic control which affect the quality of life $(\mathrm{QoL})$. Pharmacists as health worker have important role in treatment of patients with DM. The aim of the study was evaluating the effect of counselling by pharmacist to glycemic control and QoL type 2 Diabetes Mellitus (T2DM) patients. Setting and method: The study was conducted using pre-test:posttest control group design in Depok City Hospital (DCH) from October 2017 to March 2018. Respondents were T2DM outpatients and divided into intervention group and control group. Counselling by pharmacist was given after the enrolment of study. HbA1c levels and QoL was measured at the enrolment and 3 months after the study. QoL was measured with EQ-5D-5L validated instruments in Indonesian language. EQ-5D-5L consist of QoL values and visual analogue scale (VAS) score.

Main outcome measures: Outcome measures were $\mathrm{HbA1c}$ levels, QoL value and VAS score before and after counselling by pharmacist in both groups.

Results: T2DM patients fulfilled the criteria and followed the study for 3 months were 81 patients, 42 patients in intervention group and 39 patients in control group. HbA1c levels, QoL values and VAS score on intervention group at the enrolment were $7.67 ; 0.72 ; 63.45$ respectively and after 3 months $6.92 ; 0.85 ; 71.69$ respectively. HbA1c levels, QoL values and VAS score on control group at the enrolment were $8.00 ; 0.80 ; 66.14$ respectively and after 3 months $8.21 ; 0.81$; 65.50 respectively. There were significant differences in HbA1c $(p=0.004)$, QoL values $(p=0.001)$, and VAS score $(p=0.001)$ in intervention group in the enrolment and 3 months after the study, while in the control group there was no significant difference in HbA1c $p=0.591)$, QoL values $(p=0.706)$, and VAS score $(p=0.558)$.

Conclusion: Pharmacist counselling significantly affected HbAlc levels and quality of life within 3 months of study at DCH with EQ$5 \mathrm{D}-5 \mathrm{~L}$ validated instruments in Indonesian language.

Disclosure of Interest: None Declared.

HP-CE002: The medication errors cart: an educational and playful tool available to the clinical pharmacist

S. Lemay ${ }^{1, *}$, C. Chan-Fat ${ }^{2}$, B. Rutgers ${ }^{3}$, J. Gounou ${ }^{4}$, C. Pignolet ${ }^{1}$, H. Lhote ${ }^{5}$, Y. Jacques-Antoine ${ }^{3}$, I. Ramon ${ }^{1}$

${ }^{1}$ Pharmacy, ${ }^{2}$ Quality, ${ }^{3}$ Intensive care unit, ${ }^{4}$ General hospital medicine, ${ }^{5}$ Surgery, East Reunion Hospital Group, Saint Benoit, France 
Background and objective: In France, one-third of the medical errors ${ }^{(1)}$ are administration errors. Such preventable events are termed «medication errors» (ME). Most of ME are not detected in our health system and are thus not preventable ${ }^{(2)}$. Hospital staff have to gain practical knowledge of ME and of methods of prevention. Simulation is a teaching method more and more used in health system. The aim of this study was to create a medication errors cart which represents a fictitious unit cart with ME for health professionals in an Intensive unit care.

Design: This study settled in the Intensive care unit (pilot service) of a general hospital and was conducted by a clinical pharmacist (CP). A medication errors cart has been developed to help nurses to detect medication errors in the preparation of medication. Seventeen ME were designed inside the cart. The ME was typical expired drugs, homophony between drugs, «never events» related errors. The training session was not time limited and took place in the nursing stations. The CP gave guidelines on how to use the cart. An individual debriefing was done after each session.

Results: Eighteen sessions were proposed from mars to April 2018 to healthcare professional: 15 nurses and 3 student nurses. The mean time to check the whole cart was about 32 min per nurse. The EM detection rate ranged of $81 \%$ (nurses) to $92 \%$ (nurse students). Only $53 \%$ of the nurses found the «never events» related errors.

Conclusion: The medication errors cart represents for the $\mathrm{CP}$ an easy, movable, playful, useful and reusable simulation tool, to train health professional and to improve their knowledge and practical methods. This learning tool was developed in order to provide patient safety. It may also stimulate spontaneous reporting of medication errors. This successful study will be evaluated by a satisfaction questionnaire. Future sessions will be arranged in other departments.

(1) Pourrat X, Antier D, Doucet O, Duchalais A, and al. Identification and analysis of errors in prescription, preparation and administration of drugs in intensive care, medicine and surgery at the University Hospital Center of Tours (French). Presse Med 2003; 32:876-882.

(2) Le Grognec C, Lazzarotti A, Durnet-Archeray MJ, Lorcerie B. Medication Errors Resulting from Drug Preparation and Administration. Thérapie 2005; 60:391-399.

Disclosure of Interest: None Declared.

DI004: Idiosyncratic drug-induced agranulocytosis: 6 yearanalysis in a French university hospital

M. Duwez ${ }^{1, *}$, G. Szymanski ${ }^{2}$, M. Carre ${ }^{3}$, S. Chanoine ${ }^{1,4}$, P. Bedouch ${ }^{1,4}$, M. Mallaret ${ }^{5}$, M. Lepelley ${ }^{5}$

${ }^{1}$ Pharmacy Department, ${ }^{2}$ Hematology Laboratory, ${ }^{3}$ University Hematology Clinic, University Hospital of Grenoble Alpes,

${ }^{4}$ University Grenoble Alpes, ${ }^{5}$ Regional Pharmacovigilance Centre, University Hospital of Grenoble Alpes, F-38000 Grenoble, France

Background and objective: Idiosyncratic drug-induced agranulocytosis (DIA) is a rare but potentially serious haematological disorder. The pathophysiological mechanisms are multiple and poorly understood. We aimed at investigating agranulocytosis drug-related causes from the bone marrow examinations (BME) with "myeloid maturation arrest" performed in our university hospital over the last 6 years to study drug-related causes.

Design: A retrospective analysis of BME with "myeloid maturation arrest" collected for agranulocytosis (neutrophil count $\leq 0.5 \times 10^{9}$ / L) was performed from 1st January 2010 to 31th December 2016. Agranulocytosis aetiology was searched for every case. We used the French method for drug causality assessment.
Results: Among the $104 \mathrm{BME}$ performed over the 6-year study period, 41 (39.4\%) were related to DIA, whose 31 were idiosyncratic. Among these 31 cases, 27 different drugs were involved. DIA was a known adverse reaction in the summary of the product characteristics for 25 drugs (92.6\%), mainly associated with undetermined frequency $(\mathrm{n}=7)$. Mean number of suspicious drugs was 1.6 per case (range 1-3). Mean DIA onset latency was 38.1 days after starting drug (range 2-127) and mean duration of DIA was 11.3 days (range 2-60). Granulocyte growth factors (G-CSF) were used in $45.1 \%$ of cases (mean dose: $281.5 \mu \mathrm{g} /$ day) without shortening the mean duration of blood count recovery time (10.8 vs 8.2 days). Bone marrow presented hypereosinophilia in $25.8 \%$ of cases. Pharmacovigilance reporting rate was $48.1 \%$ and 13 no-reported DIA were recovered after retrospective drug causality assessment.

Conclusion: A "myeloid maturation arrest" in the BME is not pathognomonic for DIA and pathophysiological mechanisms could not be elucidated in our study. However, we recovered 13 additional cases of no-reported DIA highlighting that these rare events require multidisciplinary care involving haematologists, biologists and pharmacovigilance experts. Agranulocytosis reporting rate was high compared with usual adverse drug reaction reporting rate $(5-10 \%)$, probably related to the potential severity of this event.

Disclosure of Interest: None Declared.

DI005: Poly pharmacy patients' experience with prescribers' cooperation and adherence promoting communication skills

\section{B. Holm ${ }^{1, *}$, N. Majeed ${ }^{1}$}

${ }^{1}$ Facuty of Health Sciences, Oslo Metropolitan University, Oslo, Norway

Background and objective: Failure to adhere to medical treatment is a serious problem which may lead to substantial worsening of disease, death and increased health care costs. WHO states that $30-50 \%$ of all patients fail to follow the recommended treatment. Several studies demonstrate a connection between medication adherence and physician-patient communication. Further, risk of poor adherence increases with increasing number of pharmaceuticals, and multiple prescribers increase the risk of polypharmacy. The objective of this study was to investigate Norwegian polypharmacy patients' perspective on the issue of multiple prescribers and the degree of cooperation between their prescribers, and their adherence promoting communication skills. We wanted to understand whether the patients, after their doctors' visit, felt well enough informed in order to adhere sufficiently to their treatment.

Setting and method: 10 qualitative in-depth interviews with polypharmacy patients were performed during winter 2018 .

Main outcome measures: Systematic text condensation was used to identify major topics.

Results: Five main themes evolved during analyses: (1) communication between patient and prescribers, (2) information exchange between prescribers, (3) knowledge and beliefs, (4) adherence, and (5) GPs role. Each of these had 2-3 sub-categories. The most adherence influencing results are discussed briefly here. Co-operation: Most of the patients had more than one doctor prescribing medications. The prescribing doctors did not cooperate or communicate physically nor electronically. The patients themselves were often the main information channel between the specialist and the general practitioner (GP). Time: Almost all patients felt that they had too little time with their GPs. There was almost never enough time to discuss their medical treatment in detail. Most of the information about how to adhere to treatment was either given by the pharmacist, or the patient read up on it themselves. Beliefs: Many of the patients had certain beliefs about medicines which they did not discuss with their doctor. These beliefs influenced their adherence. 
Conclusion: Prescribers' cooperation and adherence promoting communication skills is in general experienced as poor by patients. Information on how to adhere to a medical treatment is often in the hands of the patient themselves. This demonstrates the pharmacists' important role in improving medication adherence among polypharmacy patients.

Disclosure of Interest: None Declared.

PT004: Augmented clearance in patients with colistin therapy in intensive care units

\section{A. Aitullina ${ }^{1,2, *}$, A. Krumina ${ }^{3}$, S. Purvina ${ }^{1}$ \\ ${ }^{1}$ Pharmacology, Riga Stradins University, ${ }^{2}$ Hospital pharmacy, Paul Stradins Clinical University hospital, ${ }^{3}$ Infectology and Dermatology, Riga Stradins University, Riga, Latvia}

Background and objective: Augmented renal clearance (ARC) is enhanced renal function that usually is observed in comparatively young critically ill patients without chronic organ failure. There is data that ARC could lead to sub-therapeutic concentration of some drugs, e.g. vancomycin. Colistin is potentially nephrotoxic antibiotic from polymyxin group used for treatment of severe infections in intensive care units (ICU) and it dosage depends a lot on patient renal function state.

Setting and method: Retrospective study using medical histories about adult patients admitted to Pauls Stradins Clinical University Hospital (PSCUH) ICU in 2016-2017 with diagnosed multi-drug resistant Acinetobacter baumanii infection and colistin therapy.

Main outcome measures: Colistin doses and patients demographics were compared between two groups divided according to eGFR (MDRD formula): ARC-group (eGFR more than $130 \mathrm{ml} / \mathrm{min}$ ) and non-ARC-group (eGFR less than $130 \mathrm{ml} / \mathrm{min}$ and without renal replacement therapy).

Results: Fifty-nine medical histories have met of including criteria. $41(70 \%)$ were men. Death rate was $58 \%$. Median amount of hospitalization days was 39 . The most common clinical diagnoses were COPD and pneumonia (34\%), post-myocardial infarction (15\%), subarachnoidal haemorrhage $(15 \%)$ and trauma or post-surgical complications (14\%). Twelve patients $(20 \%)$ had RRT before and during colistin therapy. Seven patients from 47 (15\%) was in ARCgroup. Only one patient from this group experience decline in renal function during colistin therapy compared with 9 patients in nonARC-group. Median age was lower in ARC group (57 vs 67 years) and median cumulative colistin dose was higher in ARC group (87 million units (MU) vs $71 \mathrm{MU}$ ), however both differences were not statistically significant. Median duration of colistin use was the same in both groups -9 days.

Conclusion: Renal function state in patients with colistin therapy is very heterogeneous so more individualized approach in dosing and monitoring could be beneficial in this patient group. Significant numbers of patients had ARC during colistin therapy, and this number could be underestimated as for renal function assessment calculator was used instead of creatinine measurement in daily urine. Preliminary results show that patients with ARC were younger and received higher cumulative colistin doses.

Disclosure of Interest: None Declared.

PT005: The efficacy of switching from Insulin Glargine $100 \mathrm{U} / \mathrm{mL}$ (Gla-100) to Insulin Glargine $300 \mathrm{U} / \mathrm{mL}(\mathrm{Gla}-300)$ in patients with type 2 diabetes mellitus

\footnotetext{
K.-H. Yang ${ }^{1, *}$, C.-T. Tseng ${ }^{1}$, L.-C. Chen ${ }^{1}$, P.-C. Chen ${ }^{1}$

${ }^{1}$ Pharmacy, Taipei City Hospital, Taipei, Taiwan, Province of China
}

Background and objective: Lantus ${ }^{\circledR}$, insulin glargine U-100 (Gla100) and Toujeo ${ }^{\circledR}$, insulin glargine U-300 (Gla-300) contain the same active ingredient and produced by the same company. Toujeo (Gla300 ), which came to market in early 2015 , is three times the concentration as compared to Lantus ${ }^{\circledR}$ (Gla-100). The aim of this study was to assess the efficacy when transferring from Gla-100 to Gla-300. Setting and method: A regional hospital in Taiwan replaced insulin glargine from Gla-100 to Gla-300 on September 1st, 2017. We screened the patients who had the same insulin glargine unit dose (IU) before and after switching. Data including HbAlc, fasting blood sugar, kidney and liver function were also collected 3 months before and after switching. After that, we kept evaluating efficacy and dose change of the poor-control-patients for 3 months.

Main outcome measures: $\mathrm{HbA1c}$, fasting blood sugar, kidney and liver function for type 2 diabetes mellitus patients' data were collected. And we collected the data of Gla-300 from poor-control patients who needed to reach the target glycemic control.

Results: There were 93 patients switching from Gla-100 to Gla-300 with the same unit dose between June and November 2017. The mean age of the patients was $66.1 \pm 13.8$ years. The average body weight was $70.9 \pm 16.8 \mathrm{~kg}$. Mean dose was $20.2 \pm 10.8 \mathrm{IU}$ per day. The data before and after switching were shown as following (Gla$100 \rightarrow$ Gla-300): a. HbA1c: $8.27 \pm 1.38 \rightarrow 8.57 \pm 1.46 \% \quad(p=$ $0.0015)$ b. fasting blood sugar: $155.6 \pm 68.1 \rightarrow 172.5 \pm 60.8 \mathrm{mg} / \mathrm{dL}$ $(p=0.011)$ c. eGFR: $61.8 \pm 42.3 \rightarrow 59.6 \pm 32.1(p=0.249) \quad$ d. ALT: $25.9 \pm 36.3 \rightarrow 24.1 \pm 23.5 \mathrm{U} / \mathrm{L}(p=0.352)$. We could see that there were no obvious change of kidney and liver function. Furthermore, 33 patients significantly had to increase the Gla-300 dose to reach the target glycemic control (dose from $20.2 \pm 10.8$ to $24.5 \pm 11.1 \mathrm{IU}, p<0.001)$ from December 2017 to February, 2018. Conclusion: This study showed that compared with Gla-100, Gla-300 patients had poorer glycemic control especially fasting blood sugar. Moreover, about 30\% patients needed higher daily dose of Gla-300 to maintain the same level of glycemic control. Therefore, pharmacists should advise doctors to monitor patients' blood sugar closely when switching Glas-100 to Gla-300 with the same dose.

Disclosure of Interest: None Declared.

PT006: Retrospective prevalence study on antimicrobial prescriptions for psychiatric inpatients of a Belgian university psychiatric hospital

L. Popleu ${ }^{1}$, M. Haesendonckx ${ }^{2}$, G. Schuurmans ${ }^{2}$, A. Schuermans ${ }^{3}$, S. Desplenter,

${ }^{1}$ Infection Control Team, UPC KU Leuven, Kortenberg, ${ }^{2}$ Academic Centre for Nursing and Midwifery, KU Leuven, ${ }^{3}$ Infection Control Team, UZ Leuven, Leuven, ${ }^{4}$ Hospital Pharmacy, UPC KU Leuven, Kortenberg, Belgium

Background and objective: Antimicrobial prescribing practice and the emergence of multi-resistant pathogens are associated. The aim of this study was to analyse the antimicrobial prescriptions practice for psychiatric inpatients.

Setting and method: A retrospective prevalence study was carried out in the psychiatric hospital UPC KU Leuven including all antimicrobial prescription (ATC code J01) for inpatients in 2016. Electronic prescriptions and electronic medical records were retrospectively checked. Ethical approval was obtained.

Main outcome measures: (1) Number of antibiotic prescriptions, proportion of patient days with antimicrobial medication. (2) Indication of antimicrobial prescriptions and type of antimicrobial medication. (3) Appropriateness of antimicrobial prescription based on at least one notification of clinical symptom (as described by the CDC (Center of Disease Control) definition of the respective infection), lab analysis (blood or urine) or radiography. 
Results: In 2016, 243 inpatients (13.5\% of all admitted inpatients in 2016) received 378 antimicrobial prescriptions. Sixty percent $(n=146)$ of included patients were female. Sixty percent $(n=144)$ were admitted to a geronto-psychiatric ward. Median age was 69 years. In $3.1 \%(\mathrm{n}=3169)$ of patient days an antimicrobial prescription was present. This number increased up to $6.0 \%$ in gerontopsychiatric wards.

Top 3 of indications of antimicriobial prescriptions were: urinary tract infections $(45.8 \%(\mathrm{n}=173))$, respiratory tract infections $(23.8 \%$ $(\mathrm{n}=90))$ and skin infections $(12.7 \%(\mathrm{n}=48))$. Amoxicillin clavulanic acid $(27.8 \% \quad(\mathrm{n}=105))$, nitrofurantoin $(19.8 \% \quad(\mathrm{n}=75))$, Amoxicillin $(12.4 \%(n=47))$ and levofloxacin $(7.9 \%(n=30))$ were the top 4 of antimicrobial medications representing $68.0 \%(n=257)$ of antimicrobial prescriptions.

Regarding the appropriateness of prescriptions: $91.7 \%(n=264)$ of prescriptions had at least one clinical symptom present. $73.4 \%(\mathrm{n}=124)$ of the 169 blood samples, $71.8 \%(n=102)$ of 142 urine samples were indicative for infection and $74.6 \%(n=44)$ of 59 thorax radiography for lower respiratory tract infections were indicative for pneumonia.

Conclusion: This is the first Belgian study on antimicrobial prescriptions in psychiatric hospitals. The prevalence of $3.1 \%$ patient days with antimicrobial medication use underlines the presence of nosocomial infections in psychiatric hospitals and the need for somatic follow-up of psychiatric inpatients. Urinary tract infections represent almost half of the infections. The choice of antimicrobial medications is in line with local antimicrobial guidelines. Procedures and education on infection management for caregivers should be embedded in all psychiatric hospitals.

Disclosure of Interest: None Declared.

PT007: Evaluation of drug-drug interactions of direct-acting antivirals in hepatitis $\mathrm{c}$ virus infected patients

L. Margusino-Framiñán ${ }^{1, *}$, A. Castro-Iglesias ${ }^{2}$, V. Giménez-Arufe ${ }^{1}$, C. Fernández-Oliveira ${ }^{1}$, P. Cid-Silva ${ }^{1}$, Á. Mena-De-Cea ${ }^{2}$, I. Rodríguez-Osorio ${ }^{2}$, B. Pernas-Souto ${ }^{2}$, M. D. Yáñez-Martínez ${ }^{1}$, S. Pertega-Díaz ${ }^{3}$, A. Sanclaudio-Luhia ${ }^{4}$, I. Martín-Herranz ${ }^{1}$

${ }^{1}$ Pharmacy, ${ }^{2}$ Internal Medicine, ${ }^{3}$ Epidemiology, ${ }^{4}$ Information Systems, A Coruña Universitary Hospital, La Coruña, Spain

Background and objective: To analyze drug-drug interactions (DDIs) of direct-acting antivirals (DAAs) identified by clinical pharmacists in hepatitis $\mathrm{C}$ virus (HCV) infected patients and to evaluate its influence on treatment effectiveness.

Setting and method: Viral Pathologies Outpatient Clinic of Hospital Pharmacy Service in a large Spanish teaching hospital.

Single-centre, prospective, cohort study approved by Regional Research-Ethic Committee, along 2.5 years. Inclusion criteria: HCV adult patients treated with DAAs \pm Ribavirin (Ledipasvir/Sofosbuvir-LDV/SOF; Daclatasvir/Sofosbuvir-DCV/SOF; Grazoprevir/ Elbasvir-GRZ/EBV; Simeprevir/Sofosbuvir-SIM/SOF; Paritaprevir/ $\mathrm{r}+$ Ombitasvir \pm Dasabuvir-PTV/OBM $\pm \mathrm{DSV} ; \quad$ Sofosbuvir/Velpatasvir-SOF/VEL) and with at least 12 weeks post-treatment followup to determin THE virologic response (SVR12). DDIs were identified and their severity categorized based on the University of Liverpool's HEP Drug Interactions Resource. Multivariate statistical analysis was carried out using t-Student, Mann-Whitney U, Chi square or Fisher's exact tests.

Main outcome measures: Baseline demographic data. Primary end points were \%patients with DDIs and distribution by: basal demographic patient variables, DAAs, therapeutic class (TC) of interacting drugs and severity. Secondary end point: \%patients with SVR12 according to presence/absence of DDIs balanced by HCV genotype, degree of liver fibrosis and previous antiviral treatment.
Results: A total of 1091 patients were included: $57.8 \pm 12.8$ years, $62.4 \%$ males, $29.9 \%$ cirrhotics, $67.6 \% \mathrm{HCV}$ genotype 1 infected, $13.4 \%$ HIV co-infected, $3.9 \%$ livers transplanted, 3.5\% hepatocarcinoma diagnosed and $70.9 \%$ naïve to DAAs. $24.8 \%$ of patients presented DDIs $\left(22.2 \% \_27.4 \%, 95 \%\right.$ CI). Older age, female gender and $\mathrm{HCV}$ genotype 1 infection $\mathrm{HCV}$ infected patients present more DDIs in the univariate analysis $(p<0.05)$. DDIs prevalence by DAAs: $37.7 \%$ PTV/OBM \pm DSV, $24.4 \%$ LDV/SOF, $16.9 \%$ GRZ/ EBV, $16.1 \%$ SOF/VEL. DDI distribution by TC: $33.6 \%$ cardiovascular, $31.3 \%$ digestive tract, $18.3 \%$ nervous system. DDIs severity: $5.3 \%$ severe, $82.2 \%$ potential, $12.5 \%$ potential-weak. RVS12 in presence/absence of DDIs was $97.5 \% / 98.6 \%$ $(p=0.256)$.

Conclusion: DDIs with DAAs were very prevalent, mainly in patients treated with PTV/OBM \pm DSV followed by LDV/SOF. A mediumhigh level of severity of DDIs was observed in this population, which led to the substitution or dose adjustment of the interacting drug. However, following the clinical recommendations contributes to maintain the high SVR12 in HCV infected patients treated with DAAs independently of their baseline variables and conditions.

Disclosure of Interest: None Declared.

\section{PT008: Modification of chronic pharmacotherapy} in perioperative period

\section{Z. Javorová-Rihová ${ }^{1,2}$, E. Puchoň ${ }^{2, *}$, M. Kuželová ${ }^{2}$}

${ }^{1}$ Department of Clinical Pharmacology and Clinical studies, Faculty hospital Trnava, Trnava, ${ }^{2}$ Department of Pharmacology and

Toxicology, Faculty of Pharmacy, Comenius University, Bratislava, Slovakia

Background and objective: Attention to the modification of chronic pharmacotherapy before planned surgery is not the same in all groups of medications and differences exist in the application of recommendations in real clinical practice. The aim of the study was to find out how the recommendations for the modification of chronic pharmacotherapy are followed before planned operations.

Setting and method: Chronic pharmacotherapy in patients hospitalized in the Faculty hospital Trnava, the Slovak Republic for planned operation was analyzed during JanuaryOctober 2017. Information on patients' medication and recommendations for its modification before surgery was taken from an anesthesiological perioperative examination available in hospital information system. Differences between published and actual recommendations from physicians in the modification of chronic pharmacotherapy prior to planned surgery in medications were evaluated.

Main outcome measures: To analyze occurrence of medications in chronic pharmacotherapy and to review differences between published recommendations and real situation in the modification of chronic pharmacotherapy before elective surgery.

Results: $72 \%(n=287 / 401)$ of patients used at least one medicine. The average number of medication used was 3.6. Medications for cardiovascular system were present in $46 \%(n=676 / 1460)$. The most commonly used medicine was acetylsalicylic acid $(5 \%, \mathrm{n}=77 / 1460)$. Antiplatelet medications, anticoagulants and medications used for therapy of cardiovascular system (except antiarrhythmic medications) have expert recommendations for modification before planned operation created by scientific societies. Based on these recommendations, treatment continued with beta-blockers $(89 \%)$ and calcium channel blockers $(50 \%)$. Therapy was discontinued in diuretics $(85 \%)$ and in medications influencing renin-angiotensin-aldosterone system (75\%). Antiplatelet, anticoagulant and antidiabetic therapy were withdrawn in all cases $(100 \%)$ but we found out differences between 
published and actual recommendations in time during which therapy should be discontinued. From the view of perioperative complications, recommendations are mainly missing in the group of antiarrhythmic medications and in medications influencing CNS.

Conclusion: There is not enough attention given to the modification of chronic pharmacotherapy before elective surgery in clinical practice. Many medications do not have recommendations available. On the other hand, not all recommendations that are available are used in clinical practice.

Disclosure of Interest: None Declared.

PH003: Technology-enabled pharmaceutical care provision to dispensing medical practices in NHS Highland: a pilot promoting equality of access to remote populations

C. Morrison ${ }^{1}$, K. MacLure ${ }^{2, *}$, K. Gibson Smith ${ }^{2}$, P. Hannam ${ }^{1}$, L. Dixon ${ }^{1}$, Y. MacRae ${ }^{1}$, S. Cunningham ${ }^{2}$, D. Stewart ${ }^{2}$

${ }^{1}$ NHS Highland, Inverness, ${ }^{2}$ Robert Gordon University, Aberdeen, United Kingdom

Background and objective: 'Achieving Excellence in Pharmaceutical Care' describes the strategic direction for inclusive, equitable access to pharmacy services across Scotland. This research aimed to evaluate the NHS Highland pilot of technology-enabled delivery of pharmaceutical care provision.

Setting and method: A mixed-methods evaluation followed 3 remotely-based pharmacists who delivered patient medication reviews to 3 GP practices (A, B, C). Patients chose between a telephone or video-link consultation using 'Attend Anywhere' software at the GP Practice or other location. Patient study packs were sent by GP Practice staff or pharmacist. The questionnaire collected data on participants' views and experiences of the pharmacist consultation using Likert scales, open questions and the CARE Measure. Participants were purposively sampled for telephone interview. Ethical approval was gained.

Main outcome measures: Participants' views and experiences of the pharmacist consultation.

Results: Thirty-three questionnaires were returned, representing practice $\mathrm{C}(\mathrm{n}=23,69.7 \%), \mathrm{A}(\mathrm{n}=5,15.2 \%), \mathrm{B}(\mathrm{n}=5,15.2 \%)$. All consultations were via telephone $(\mathrm{n}=33,100 \%)$. Median age of respondents was 66 years; most were female $(\mathrm{n}=19,57.6 \%)$; and, retired $(\mathrm{n}=23,69.7 \%)$. Respondents reported the pharmacist was 'very good' or 'excellent' at 'making them feel at ease' $(n=30$, $90.9 \%)$, 'letting them tell their "story"' $(\mathrm{n}=28,84.8 \%)$, 'listening' $(\mathrm{n}=25,75.8 \%)$, 'being interested in them as a whole person' ( $\mathrm{n}=27,81.8 \%)$, 'understanding concerns' $(\mathrm{n}=26,78.8 \%)$, 'showing care and compassion' $(\mathrm{n}=26,78.8 \%)$, 'being positive' $(\mathrm{n}=29$, $87.9 \%)$, 'explaining things clearly' $(\mathrm{n}=29,87.9 \%)$, 'helping them take control' ( $\mathrm{n}=27,81.8 \%)$ and 'making a plan of action' $(\mathrm{n}=24$, $72.7 \%$ ). The majority agreed that they would 'recommend consulting a pharmacist to others' $(n=20,60.6 \%)$, however, also agreed that they 'would prefer to consult a GP' $(\mathrm{n}=20,60.6 \%)$. Eight interviews $(\mathrm{A}, \mathrm{n}=3,37.5 \% ; \mathrm{B}, \mathrm{n}=5,62.5 \%)$ were conducted. To date, feedback has been positive with interviewees highlighting that the consultation was convenient and helpful. Instability of the internet connection was cited as a barrier to utilising video-link consultations, '...internet connection here is not necessarily brilliant so I think the phone really was the only option'.

Conclusion: These findings highlight the patient perceived value of providing medication reviews by remotely-based pharmacist which may address issues in accessing medicines-related care from remote areas. However, Scotland's internet infrastructure remains unreliable so the new 'Attend Anywhere' software was not fully tested.

Disclosure of Interest: None Declared.
PH004: Telecare provision in Northern Ireland

H. J. Al-Obaidi',*, S. Al-Azzam ${ }^{2}$, V. Faith ${ }^{1}$, E. Gardner ${ }^{3}$, J. C. McElnay ${ }^{1}$

${ }^{1}$ School of Pharmacy, Queen's University Belfast, Belfast, United Kingdom, ${ }^{2}$ School of Pharmacy, Jordan University of Science and Technology, Irbid, Jordan, ${ }^{3}$ Northern Ireland Clinical Trials Unit., Belfast, United Kingdom

Background and objective: Pharmacists are increasingly involved in the care of elderly patients and as such need to be familiar with other types of care being availed of by this population. Telecare is a service which provides a range of IT support activities to assist people (mainly elderly) to live independently in their own homes. It involves the use of sensors which transmit alerts, e.g. falls, to a central coordinating centre. The study objective was to (a) construct a descriptive overview of the telecare programme in N. Ireland, and (b) record hospital based service usage by patients pre- and postinstallation of telecare equipment.

Setting and method: Ethical and governance approvals were obtained from the relevant authorities. Telecare service usage data, over the period February 2010-February 2016, were obtained from the service provider and were linked to healthcare utilisation data, at patient level, prior to analysis.

Main outcome measures: Demographgic data for patients who recieved the telecare service across five HSC Trusts in N. Ireland. Number of equipment, number of contact calls by age group. Hospital based service usage record by patients pre- and post-installation of telecare equipment.

Results: A total of 2387 patients had telecare services initiated; linked healthcare utilisation data were available for 1883 individuals. The mean age of patients was $78(\mathrm{SD} \pm 12)$ years (median of 81 years). More females $(67.7 \%)$ than males $(32.3 \%)$ received the service. Participants had $1-10$ items of equipment installed in their homes (mean 2.6; median 2). The number of 'calls' to the co-ordinating centre was highest in patients $\geq 85$ years old (mean of 86 calls per year). Calls were evenly distributed by gender. Non-elective hospital admissions, length of hospital stay, emergency room visits and outpatient clinic visits were all higher post-installation of the telecare equipment.

Conclusion: Despite likely benefits of telecare, e.g. peace of mind in patients and their relatives, healthcare utilisation significantly increased post enrolment in the service, which may simply reflect the increasing healthcare needs over time in an ageing population. It is therefore recommended that a randomised, controlled trial is initiated to fully evaluate the potential of telecare services to improve clinical, humanistic and economic outcomes across N. Ireland.

Disclosure of Interest: None Declared.

RD001: An exploration of social media and eprofessionalism in pharmacy practice

A. Brown ${ }^{1, *}$, D. Stewart ${ }^{1}$, S. Pedersen ${ }^{1}$, K. MacLure ${ }^{1}$, B. Addison ${ }^{1}$

${ }^{1}$ Robert Gordon University, Aberdeen, United Kingdom

Background and objective: To date, the literature on the use of social media (SoMe) within pharmacy has a distinct lack of focus on use by registered pharmacists, with the main body of evidence relating to pharmacy students. Literature suggests a need for professional guidance to support individuals in their use of social media, with references being made to eprofessionalism and fitness to practise. A systematic review (SR) carried out by the research team showed a lack of consistency of approach to professional guidance and a focus on what not to do rather than shedding light on appropriate online behaviours. In addition, caution is advised with regards 
to social media use and, in particular, with regard to blurred boundaries between personal and professional personas.

This study sought to explore how eprofessionalism and 'appropriate' online behaviour is defined and characterised and to explore when online behaviour 'crosses the line' and becomes 'inappropriate'.

Design: Four activity based workshops were conducted at two pharmacy conferences (BPSA, HSRPP) and two Royal Pharmaceutical Society workplace venues. Activities were developed around defining eprofessionalism, gauging personal/professional use of social media, exploring (in)appropriate behaviours and responding to case scenarios. Each was informed by existing literature, a previous SR and pilot study. Themes were inducted from analysing the textual content of the focus groups and responses to other activities collated and synthesised. The research was approved by the School of Pharmacy and Life Sciences Ethical review panel.

Results: Focus groups were conducted across four Master of Pharmacy (MPharm) stages ( $\mathrm{S} 1: \mathrm{n}=10 ; \mathrm{S} 2: \mathrm{n}=5 ; \mathrm{S} 3: \mathrm{n}=8 ; \mathrm{S} 4: \mathrm{n}=6$ ). All except one participant $(n=29)$ were aged under 24 with near equal male:female ratio $(n=15: 14)$. The majority of participants did not use SoMe guidelines despite daily SoMe use, but rather used personal judgement to decide on appropriate SoMe content. Some elements of existing guidance were seen as valuable but lacked balance of content/tone and examples of appropriate SoMe behaviours. There was no agreement on 'appropriate behaviours' however general concerns emerged around guidance impinging personal personas and impacting perceptions of e-professionalism.

Conclusion: The lack of shared definition of eprofessionalism and appropriateness of online behaviours highlights the need for a clearer understanding amongst healthcare professionals and the wider population. With no consensus on how to engage positively with SoMe or where the line between (in)appropriate lies further research will explore this and inform recommendations on the provision of guidance.

Disclosure of Interest: None Declared.

\section{Posters}

CP-PC014: Medication related experiences of adults with learning disabilities

\section{J. Macleod ${ }^{1, *}$, K. MacLure ${ }^{1}$ \\ ${ }^{1}$ School of Pharmacy, Robert Gordon University, Aberdeen, United Kingdom}

Background and objective: Learning disabilities (LD) is a term used to describe an individual who: has significant impairment of intellectual functioning; has significant impairment of adaptive functioning; and where the age of onset was before adulthood ${ }^{1}$. People with LD are known to have increased medical needs and are known to be prescribed more medication than the general population ${ }^{2}$. Little is known about their experiences with medication ${ }^{3}$. The aim of this research was to explore the medication related experiences of adults with LD using the Patient's Lived Experience with Medicines (PLEM) conceptual model ${ }^{1}$.

Setting and method: After the required ethical approval, qualitative, case-study methodology was used to explore the experiences of 10 adults with LD with medication. These adults were all living in the community in the north-east of Scotland. Data was collected through interviews, observation and review of any care provider records.

Main outcome measures: Identifying:

- What medication related burden is/has been experienced by adults with LD?

- What are/have been the medication related beliefs of adults with LD?
- What is/has been the medication taking practice of adults with LD?

Results: What medication related burden is/has been experienced by adults with $L D$ ?

- De-sensitisation to the burden of medication is apparent

- Adverse effects from medication on cognitive ability, mental wellbeing, and social activity are of particular concern

- Changes to medication and/or routine can be challenging

- Burden is often transferred to carers

What are/have been the medication related beliefs of adults with LD?

- Capacity and level of capability determines the beliefs of the adult with LD and the influence of others

- Medication is perceived to be beneficial and necessary

- Health care professionals (HCPs) are seen to be the medication experts but the carers are the experts on the adult with LD.

- With support and/or supervision, adults with mild LD can be enabled to self-administer.

What is/has been the medication taking practice of adults with LD? Adults with LD are generally compliant with prescribed medication but regimens have often been optimised by carers.

Conclusion: Through case study methodology, this research has outlined the medication related experiences of adults with LD. This increased understanding will allow HCPs, in particular pharmacists, to better support adults with $\mathrm{LD}$ in relation to medication.

Disclosure of Interest: None Declared.

HP-PC022: Practice assessment of antibiotics combinations: more does not mean better

P. Alexandrzak ${ }^{1, *}$, L. Painchart ${ }^{1}$, C. Lu ${ }^{1}$, N. Van Grunderbeeck ${ }^{2}$, B. Frimat $^{1}$, C. Fournier ${ }^{1}$

${ }^{1}$ Pharmacy, ${ }^{2}$ Infectious diseases unit, Centre hospitalier de Lens, Lens, France

Background and objective: To evaluate prescription practices regarding antibiotic combinations in our hospital, and their conformity towards local guidelines.

Design: Elaboration of an evaluation chart (data related to drugs were searched: indications, doses, traceability of the treatment reassessment...); 6 days of data collection in medicine and surgery units (all patients of each unit receiving more than one antibiotic were included on a given day); analysis of the antibiotic combination's and treatment length's conformity by a pharmacist and an infectious disease specialist.

Results: 21 patients hospitalized in 10 different units were treated with at least two antibiotics. These patients received an average of 2.1 antibiotics during 7.9 days. The infection was documented for 3 (14\%) patients, the $18(86 \%)$ others were treated according to a probabilistic approach. For $18(86 \%)$ patients, an association of antibiotics was recommended (febrile neutropenia, sepsis, aspiration pneumonia, endocarditis, bone and joint infection...); the prescribed association was consistent with the guidelines for $15(83 \%)$ of them. An infectious disease specialist made treatment recommendations for $7(33 \%)$ patients; all of them were in compliance with the guidelines. $17(81 \%)$ patients received an appropriate dosing; for the $4(19 \%)$ others, a pharmaceutical intervention was made, leading to a dose modification for 3 of them. The treatment length was $>7$ days for 12 $(57 \%)$ patients; for $4(33 \%)$ of them, this treatment duration wasn't justified. Antibiotic treatment reassessment after $48 \mathrm{~h}$ was indicated in the medical file for 7 (33\%) patients. 
Conclusion: This study shows deviations between professional practices and local guidelines in our hospital: unjustified antibiotic combinations, discrepancies between prescribed and recommended associations, excessive length of treatment, lack of traceability of the treatment reassessment. These results will be passed on to the prescribers to raise awareness regarding appropriate antibiotic use: antibiotics mustn't be used as tranquilizers for physicians; an antibiotic combination must be justified and reassessed. The pharmacist's implication must be constant: pharmaceutical interventions regarding doses, treatment lengths and $48 \mathrm{~h}$ reassessment are key elements for a wise use of antibiotics, a crucial issue in a time of increasing bacterial resistances.

Disclosure of Interest: None Declared.

\section{HP-PC024: Medication reconciliation at admission} in an orthopedic-traumatologic surgery ward: setting up, benefit and perspective

\section{A. Barbier ${ }^{1, *}$, F. Koussougbo ${ }^{2}$, N. Guénault ${ }^{1}$ \\ ${ }^{1}$ Pharmacy, ${ }^{2}$ Orthopedic-Traumatologic, CH Armentieres, Armentières, France}

Background and objective: Securing and optimizing patient's care in health care institution have become major concerns in public health. Surgery wards are considered at risk of medication errors $(\mathrm{EM})$, it is then interesting to strengthen the pharmaceutical presence. The objective is to implement the medication reconciliation at admission (MRA) in an orthopedic-traumatologic surgery ward (OTSW) with an evaluation of prescription discrepancies and risk factors associated to their apparition.

Design: Prospective and single-center study over 5 months. Have been included patients aged 50 or older admitted for a schedule hospitalization or by emergencies. MRA has been done retroactively. An analysis of unintentional discrepancies (NID) has been done descriptively and statistically.

Results: 161 MRA done of which 123 patients admitted by emergency and 38 for a scheduled hospitalization. $70 \%$ of women and $30 \%$ of men, an average age of 77 years old and an average length of stay of 7.5 days. The average length of a MRA was $27 \mathrm{~min}$. The average time between the patient's arrival and the realization of MRA was 1.5 day. 1495 prescriptions was analyzed and 952 discrepancies identified of which 268 (28.2\%) NID (average of 1.66 NID per patient). The NDI correction rate was $81 \%$. Being admitted by emergency multiplies by 2 the number of NID $(p=0.005)$. The number of drugs at home also influences the NID number: taking more than 8 drugs multiplies by 2.8 the number of NID $(p<0.001)$. It is the same for the time between the admission and the MRA: an additional day increases the number of NID of $16 \%(p=0.03)$. The most frequent type of NID is the stop of a drug $(69 \%)$. The ATC classifications mainly aimed was cardiovascular, nervous and digestive systems. The grading of NID seriousness, made by a pharmacistsurgeon pair, shows the following distribution: $46 \%$ of minor NID, $48 \%$ of significant NID and $6 \%$ of major NID. For each additional NID on a prescription, the probability of seeing appear a significant or major $\mathrm{g}$ NID is multiplied by $3(p<0.0001)$.

Conclusion: It seems necessary to pay attention of prescription of patients admitted by emergency, especially if they have a lot of drugs in order to avoid significant or major gravity NID. NID persistence for scheduled patients shows the interest of pharmacist during the preanesthetic consultation. The correction rate of $81 \%$ must be considered in relation to difficulties encountered in a surgery ward (poor adhesion to MRA; frequent turn-over of anesthetists; surgeons unconcerned by the prescription of the patient's usual treatment...).
Our study shows that MRA partially helps to prevent the appearance of ME in OTSW, and has a potential effect on length of hospital stay or on rehospitalization.

Disclosure of Interest: None Declared.

\section{HP-PC025: Fluconazole: «high-risk drug»?}

A. Bernard-Arnoux ${ }^{1, *}$, G. Rousseaux ${ }^{1}$, P. Quillet $^{1}$, C. Mongaret ${ }^{1}$, M. Bonnet ${ }^{1}$, M. Legrand ${ }^{1}$, D. Hettler ${ }^{1}$

${ }^{1} \mathrm{CHU}$ Robert Debré, Reims, France

Background and objective: To secure the medical care process, a "high-risk drugs list", defined in our establishment, is used by pharmacists to prioritize the pharmaceutical analysis of prescriptions. Due to several medication errors, we are wondering about the interest of adding fluconazole to this list.

Design: A retrospective study was conducted over 4 weeks in our university hospital (2500 beds), from computerized prescriptions or paper prescriptions including fluconazole. Demographic, clinical, biological and therapeutic data relating to the patients were collected using a structured and standardized data collection form.

Results: Twenty-nine patients were prescribed fluconazole: 13 men and 16 women (sex-ratio $=0.81$ ) with an average age of 66.7 years [16-92] and an average BMI of $24.1 \mathrm{~kg} / \mathrm{m}^{2}$ [15.4-43.7]. 8/29 patients had impaired renal function: moderate $(n=5)$ or severe $(n=3)$ renal impairment. 9/29 patients suffered from swallowing disorders. Indications for fluconazole treatment were: systemic $(\mathrm{n}=5)$, urinary $(\mathrm{n}=6)$, oral $(\mathrm{n}=6)$, and other or unrecorded $(\mathrm{n}=12)$ candidiasis. Fluconazole was administered intravenously to $12 / 29$ patients, 4 of whom had known swallowing disorders. $5 / 29$ patients should receive a loading dose (indicated in systemic candidiasis) but only one received a loading dose. 5/8 patients with renal impairment had appropriate dosage according to recommendations. Only one patient (with severe renal impairment and BMI $=19.4 \mathrm{~kg} / \mathrm{m}^{2}$ ) had a plasma drug monitoring (residual concentration $<80 \mathrm{mg} / \mathrm{L}$ ). One case of drug interaction between fluconazole and apixaban was observed with increased risk of bleeding by increasing apixaban plasma concentrations.

Conclusion: Our study showed several medication errors related to fluconazole prescriptions, in particular: non-compliance with the loading dose, inappropriate route, absence of plasma drug monitoring especially for extreme weights, drug interactions. Our study highlights the importance of including fluconazole on the "high-risk drugs list" to prioritize pharmaceutical analysis of fluconazole prescriptions and promote the good prescribing practices.

Disclosure of Interest: None Declared.

\section{HP-PC026: The development and reliability of a Clinical Pharmacy Triage Tool in the Emergency Department}

\section{J. Blackburn-Smith ${ }^{1, *}$, A. Lawther ${ }^{1}$, K. Hodson ${ }^{2}$}

${ }^{1}$ Pharmacy Department, South Eastern HSC Trust, Dundonald,

${ }^{2}$ School of Pharmacy and Pharmaceutical Sciences, Cardiff University, Cardiff, United Kingdom

Background and objective: Increasing complex patient populations have led to an increase in demand on the health service and clinical pharmacy resources. The design and development of a clinical pharmacy triage tool for use in the Emergency Department aimed to stratify patients based upon their clinical pharmacy needs in order to prioritise workload and use resources effectively.

Design: A 3-phase mixed-methods design and literature review was used to allow for data triangulation and evaluation. Initial triage tool development followed semi-structured key informant interviews, 
utilising action research methodology for the development of the final triage tool. Cohen's kappa reliability methodology determined the reliability of the clinical pharmacy triage tool when used live by clinical pharmacists in the Emergency Department.

Results: Data demonstrated that medication, disease and patientcentred factors are all equally important in developing a robust clinical pharmacy triage tool that can be used to stratify individual patient clinical pharmacy needs in the Emergency Department. Substantial $(\kappa=0.65)$ and almost perfect $(\kappa=0.86)$ levels of agreement demonstrated significant reliability across the team of Emergency Department clinical pharmacists when using the tool. This has led to the routine use of clinical pharmacy triage in this area; ensuring highrisk patients are seen and reviewed by a clinical pharmacist in a timely manner.

Conclusion: Significant reliability data has demonstrated the potential benefits clinical pharmacy triage can have in terms of prioritising patient needs and resources within the Emergency Department. Further implementation and evaluation of clinical pharmacy triage in other acute areas and wards may help redesign how clinical pharmacy services can be delivered, leading to improved patient care and safety. Disclosure of Interest: None Declared.

HP-PC027: Evaluation of the management of drugs batch recalls E. Coulpier ${ }^{1, *}$, J. Courseaux ${ }^{1}$, S. Bouillon ${ }^{1}$, E. Morice ${ }^{1}$, R. Delplanque ${ }^{1}$

${ }^{1}$ 076, Hôpital Jacques Monod - Pharmacie, Montivilliers, France

Background and objective: The management of drugs batch recalls is the subject of a procedure to quickly manage National Agency for Medicines alerts in the pharmacy and in the units care (UC). At the pharmacy, a check-list can be used to help with the withdrawal management. The documents are archived in a binder and a computer table. To inform the UC, a standard letter with a reply card is sent to the health executives by an internal mailbox. To secure the medical treatment, we wanted to check the application and the effectiveness of the procedure.

Setting and method: In order to set up a retrospective audit, three drugs recalls, which were used across the institution, were selected from those that occurred in 2017. All hospital units and the pharmacy were audited over 4 half days by a pharmacy intern and a nurse.

Main outcome measures: Two collection grids were created as well as a questionnaire self-administered for the pharmacy dispensers.

Results: On the three withdrawals, only 3 capsules of an impacted batch were found in the medicine cabinet of a unit despite the return of the reply card mentioning the absence of the batch. Less than $40 \%$ of the UC returned the card to the pharmacy. For these, no reminder had been sent. Regarding the transmission of information in the UC: for $68 \%$, the health executives relayed information to the nurses who checked the presence of the batch, for $25 \%$ the executives managed the withdrawal alone, for $6 \%$ the system was unknown. At the pharmacy, none of the affected batches were found and one check-list was missing.

11 out of 24 dispensers answered the questionnaire. During a drug reintegration, $82 \%$ never checked the computer list of withdrawals due to lack of time, omission or lack of knowledge of which file to check.

Conclusion: Following these results, several areas of improvement were identified: harmonizing the drugs labels between the alert and the medicine cabinet, simplifying the computer list, communicating the results in a management meeting to raise the awareness for all parties.

A second audit round will be conducted to check the effectiveness of these actions.

Disclosure of Interest: None Declared.
HP-PC028: CYP2C19*17 polymorphism in stroke or transient ischemic event patients

X. Díaz-Villamarín', C. L. Dávila-Fajardo,

D. Blánquez-Martínez ${ }^{1}$, E. Leno-Durán ${ }^{2}$, A. Antúnez-Rodríguez ${ }^{2}$, M. Pérez-Campos ${ }^{1}$, I. Cano-Talavera ${ }^{1}$, R. Herrera-Trigueros ${ }^{1}$, R. Álvarez-Sánchez ${ }^{1, *}$, L. J. Martínez-González ${ }^{2}$

${ }^{1}$ Clinical Pharmacy, San Cecilio University Hospital, ${ }^{2}$ Genomics Unit, GenYo, Granada, Spain

Background and objective: Clopidogrel is metabolized by the CYP2C19 isoenzyme to its active metabolite responsible for the inhibition of platelet aggregation. Different mechanisms have been proposed to explain interindividual differences on clopidogrel response, among them, CYP2C19 polymorphisms have shown the highest level of evidence in acute coronary syndrome (ACS) patients. The CYP2C19*17 allele is related with an ultra-rapid metabolism (UM) phenotype, this means an enzyme (CYP2C19) gain of function (GOF), so, higher clopidogrel antiplatelet effect.

The aim of this study is to evaluate the CYP2C19*17 SNP influence on clopidogrel response in stroke or transient ischemic event (TIA) patients.

Setting and method: Retrospective cohort study. Patients treated with clopidogrel after stroke or TIA were recruited and dichotomized depending on their CYP2C19 gain of function (GOF; CYP2C19*17 carriers) status.

Main outcome measures: The primary endpoint was the occurrence of the combined cardiovascular (CV) death, stroke or TIA, and acute coronary syndrome (ACS) during clopidogrel treatment from the recruitment until the treatment was stopped or 31st July 2017.

Results: 67 patients were recruited, all of them were Caucasians, mean age $68.22( \pm 9.83)$ and $24(35.82 \%)$ were women. The most of them were recruited due to stroke $(n=53 ; 79.1 \%)$, only $14(20.9 \%)$ because of a TIA. 19 patients $(28.4 \%)$ were heterozygous and 5 (7.5\%) recessive homozygous, total 24 (35.8\%) CYP2C19 GOF patients. Among our patients, $11(16.4 \%)$ presented stroke/TIA, 2 (3.0\%) ACS and 1(1.5\%) CV death; in total, 14 (20.8\%) individuals met primary endpoint during clopidogrel treatment. Carrying the CYP2C19 GOF allele (Genotypes: CYP2C19*1/*17 or CYP2C19*17/*17) was associated to the response (OR $=0.11 ; 95 \%$ $\mathrm{CI}=0.02-0.79 ; p=0.012$ ).

Conclusion: The CYP2C19*17 allele protects against the occurrence of major $\mathrm{CV}$ events.

Disclosure of Interest: None Declared.

HP-PC030: A retrospective regional audit of compliance with urinary tract infection: treatment guidelines in secondary care

L. Hagan ${ }^{1, *}$, A. Brady ${ }^{1}$, C. Mallon ${ }^{1}$

${ }^{1}$ Pharmacy Department, Belfast Health and Social Care Trust, Belfast, United Kingdom

Background and objective: Recent strategies to tackle antimicrobial resistance have identified antimicrobial stewardship as a means to encourage prudent prescribing. Furthermore, Public Health initiatives such as the Strategy for Tackling Antimicrobial Resistance (STAR 2012-2017) cite the establishment and maintenance of systems to monitor antimicrobial usage and surveillance of resistance as a key objective $^{1}$. As such, this study focuses on urinary tract infections (UTI), at present the second most common indication for empirical antimicrobial treatment in both primary and secondary care ${ }^{2}$.

Design: The study is a retrospective audit across the five Health and Social Care Trusts in Northern Ireland. A total of 303 patients, with a diagnosis of uncomplicated, complicated or catheter associated UTI, 
were randomly selected from all patients admitted from January to August 2016. The primary aim was to assess compliance with empirical guidelines for the treatment of uncomplicated, complicated and catheter associated UTI. Secondary aims focussed on documentation of clinical symptoms, obtaining and recording of appropriate cultures and documenting intended duration or review date of antibiotic therapy.

Results: Overall Trust-wide compliance with regional guidelines was $31 \%(\mathrm{n}=95)$. Of 303 patients reviewed $57 \%(\mathrm{n}=173)$ were prescribed an antibiotic compliant with regional guidelines. Two of the hospital trusts met the Trust-wide target of $95 \%$ compliance for correctly prescribed dose of antibiotic. Of 303 patients reviewed, 54\% had a documented review date or duration on their Kardex and $42 \%$ $(n=129)$ had a documented review or duration in their medical notes.

Conclusion: In conclusion, none of the five Trusts met the Trust-wide agreed target of $95 \%$ compliance to regional guidelines. Based on the findings of this audit the following recommendations are proposed to improve compliance to the guidelines, promotion of the regional guidelines, revising the regional Kardex to include a dedicated section for recording intended duration of antibiotic, development of regional evidence based algorithm to aid diagnosis and classification of UTI, education for the management of asymptomatic bacteria, and education on using urinalysis and culture results to guide treatment.

\section{References}

1. Department of Health, Social Services, and Public Safety. Strategy for Tackling Antimicrobial Resistance (STAR) 2012-2017.

2. Scottish Intercollegiate Guidelines Network. Management of suspected bacterial urinary tract infections in adults: a national clinical guideline. Edinburgh: SIGN; 2012.

Disclosure of Interest: None Declared.

HP-PC031: Multidisciplinary collaboration in the establishment of a desensitization protocol: platinum chemotherapy hypersensitivity case

A.-S. Mangé $^{1, *}$, T. Barsagol ${ }^{1}$, M. Carreiro ${ }^{2}$, A. Staub $^{2}$, D. Giamarchi ${ }^{3}$, V. Bassoua ${ }^{1}$

${ }^{1}$ Pharmacy, ${ }^{2}$ Department of oncology, ${ }^{3}$ Department of pneumology and allergy, Centre Hospitralier Montauban, Montauban, France

Background and objective: Platinum-based chemotherapy (PC) is the standard therapy for many cancers treatment. In patients with recurrent disease and multiple lines of PC hypersensitivity may occur. The initiation of a desensitization protocol can be considered.

The objective is to assess the benefit and safety of desensitization protocol in a platinum chemotherapy hypersensitivity case.

Design: 74 years old patient with a serous carcinoma of the ovary, presented in 2004, 2008 and 2012 three episodes of clinic evolution treated by surgery and adjuvant chemotherapy (carboplatin-paclitaxel). She had two shocks suggesting to anaphylaxis during carboplatin's administrations in 2009 (to the fifth cycle) and 2012 (to the second cycle).

In 2017 we discovered a recurrence with tumour mass of 3-4 cm and several secondary liver injuries. It's inoperable and given the platinum sensitivity and the somatic BRCA genotype, the best therapeutic option was PC. A gemcitabin-oxaliplatin protocol was chosen during a multidisciplinary meeting, because of the cross-reactivity risk and an acute renal failure that contraindicate the cisplatin use.

Skin testing with Prick Test and Intradermal Test (to carboplatin, cisplatin and oxaliplatin) performed by the allergy specialist, to recommended dilutions, were negative. But given the strong suspicion of allergy, oxaliplatin was reintroduced with 13-step desensitization procedure.

The pharmacist was involved in drafting a protocol including the preparation of three oxaliplatin solutions diluted to concentrations of $0.01 \mathrm{mg} / \mathrm{ml}, 0.1 \mathrm{mg} / \mathrm{ml}$ et $1 \mathrm{mg} / \mathrm{ml}$ and its establishment. Initial desensitizations are carried out in intensive care unit.

Results: After 5 cycles:

- The chemotherapy is well tolerated. After the third cycle, the administration is performed in a conventional unit care.

- The follow-up shows a good response to the treatment. The thoracic-abdominal-pelvic scan shows a regression of liver injuries (around 20\%), a stabilization of the iliac tumor and a small increase in size of adrenal tumor. The protocol is continued.

Conclusion: Desensitization protocol is safe and effective. The pharmacist contribution in the multidisciplinary collaboration is here significant for the establishment of a new chemotherapy protocol.

Disclosure of Interest: None Declared.

HP-PC032: Optimizing medication reconciliation: creation of a shared electronic tool

J.-F. Meyer ${ }^{1}$, A.-L. Mouterde ${ }^{2}$, D. Toulet ${ }^{3}$, E. Salmi ${ }^{2}$, I.-B. Balde ${ }^{1}$, S. Combaud ${ }^{3}$, C. Michel $^{3}$, V. Rathouin ${ }^{2}$, R. Ratiney ${ }^{3}$, J.-E. Fontan ${ }^{1 * *}$, A. Jacolot ${ }^{2}$

${ }^{1}$ Pharmacy, Hôpital Jean Verdier (AP-HP), Bondy, ${ }^{2}$ Pharmacy, Hôpital Avicenne (AP-HP), Bobigny, ${ }^{3}$ Pharmacy, Hôpital RenéMuret-Bigottini (AP-HP), Sevran, France

Background and objective: Medication reconciliation (MR) is an effective process for the detection of medication discrepancies during the care transitions process. Health care professionals collaborate together with the patient and his family to improve medication safety. The care transition process in our hospital group is articulated between three hospitals. MR at admission and discharge is realized for the elderly patients ( $>65$ years) and/or treated by chemotherapy. Pharmacy students, residents and also medicine students are actors of the process, under the oversight of a pharmacist.

The purpose of our project is the creation of a shared and secured electronic tool, not only to permit to standardize our practices and documents but also to improve the communication between our three hospitals and with the community pharmacies.

Design: The Regional Health Agency of Ile-de-France funded the project in the purpose of promoting the MR. The tool has been developed on Microsoft Access ${ }^{\circledR}$ by a medical secretary and a pharmacist. The protection of its data has been validated by the National Commission of Computing and Freedoms. The drugs database Theriaque $^{\circledR}$ has been implemented. The contact information of community pharmacies are registered in the tool and permit to establish a collaborative information flow with medication information at the discharge.

Results: The development lasted from January 2017 to March 2017, before the release of the first version. Personal data useful for MR (e.g. name, age, hospitalization date, reason for hospital admission, name of the community physician and pharmacist...) are contained in the database. The whole prescription is included with discrepancies detected (intentional or not) and gravity evaluation. The performing time and sources used are also informed. All information is available for every professional trained. Instructions for use and regular training sessions are established. Edition of a record of reconciliation with all information cited above is automatic. The record is incorporated in the medical record and sends to health care professionals at the discharge. 
Conclusion: From April 2017 to March 2018, 580 prescriptions have benefited the MR at admission and 38 at discharge. 35 health care professionals ( 8 pharmacists, 1 pharmacy resident, 8 pharmacy students and 18 medicine students) have been trained. The development requires continued attention and is still ongoing, new versions have been developed with implementation of new features (improved ease of use, statistics of MR...). This shared electronic tool has permitted a standardization of the process of MR between the three hospitals. Shared information is primordial for security of the care process of the patient in our hospital group and with other health care professionals. The next steps would be to evaluate the effects on the quality and safety of healthcare, and then to incorporate this tool in the prescription software.

Disclosure of Interest: None Declared.

\section{HP-PC033: Economic impact of biosimilar etanercept use}

\section{R. Morón ${ }^{1}$, P. Nieto Gómez¹, C. Garcia Fernandez ${ }^{1}$,}

R. Alvarez Sanchez ${ }^{1, *}$

${ }^{1}$ Farmacia Hospitalaria, Hospital Universitario Clinico San Cecilio, Granada, Spain

Background and objective: The prescriptions of biologics are increasing as new indications and drugs are authorized. Since biosimilar drugs were introduced in the pharmaceutical market, they became an alternative to continue with the demand at competitive costs.

To analyse the economic impact from incorporating the biosimilar etanercept in the hospital pharmacotherapeutic guide.

Setting and method: Retrospective observational study in a third level hospital. The study period goes from April 2017 to April 2018.

Main outcome measures: The database are obtained for the dispensing program. The selected patients were the all patients treated with etanercept biosimilar since its incorporation into the pharmacotherapeutic guide of the hospital. The studied variable is the savings derived when using the biosimilar drug compared to the original drug. Results: The study included 30 patients from Rheumatology service. A total of 533 administrations of etanercept biosimilar were performed in the 9-month study period. The price of the biosimilar drug vial is $92.11 €$ whereas the original drug is $176 €$, being $44,713.37 €$ the economic saving produced in the administration of etanercept biosimilar in 9 months.

Conclusion: The biosimilar drugs market is growing which leads us to conduct studies in daily clinical practice, both in terms of efficacy and safety as well as economic saving. In the study carried out in our pharmacy service, an economic advantage of the biosimilar drug against the original drug is clearly demonstrated. It is important to find efficient treatments for the public health system.

Disclosure of Interest: None Declared.

HP-PC034: Efficacy and safety of cinacalcet in secondary hyperparathyroidism to chronic renal insufficiency

M. Prieto Castelló ${ }^{1, *}$, C. Aparicio Rubio ${ }^{1}$, S. Cornejo Uixeda ${ }^{1}$, E. Monteagudo Santolaya ${ }^{1}$, A. Sánchez Alcaraz ${ }^{1}$, B. Quintana Vergara ${ }^{1}$

${ }^{1}$ Pharmacy, Hospital Universitario de la Ribera, Valencia, Spain

Background and objective: Evaluate the efficacy and safety of cinacalcet in patients with hyperparathyroidism secondary to chronic renal failure without renal replacement therapy or kidney transplantation.

Design: Retrospective observational study of patients with hyperparathyroidism secondary to chronic renal failure under treatment with cinacalcet.
The following variables were collected from the electronic medical record: age, sex, weekly dose of cinacalcet at the beginning of treatment and currently, levels of parathyroid hormone (PTH), calcium, phosphorus, albumin, creatinine and glomerular filtration at the beginning of treatment, at 12 months and 24 months after starting treatment.

Results: 21 patients were included, 11 women (52.4\%) and 10 men $(47.6 \%)$ with an average age of 75 years (58-86). The mean weekly dose of cinacalcet at the start of treatment was $176.5 \mathrm{mg}(60-420)$. Stage III renal failure was the most common (48\%) followed by stage IV $(33 \%)$.

Mean analytical values at the start of treatment with cinacalcet were: $\mathrm{PTH}=266.4 \pm 190.5 \mathrm{pg} / \mathrm{ml} ; \quad \mathrm{Ca}=10.5 \pm 0.9 \mathrm{mg} / \mathrm{dl} ; \quad \mathrm{PO} 4-$ $=3.2 \pm 0.97 \mathrm{mg} / \mathrm{dl} ; \quad$ Albumin $=4.3 \pm 0.28 \mathrm{mg} / \mathrm{dl} ; \quad$ Serum creatinine $=1.8 \pm 1.4 \mathrm{mg} / \mathrm{dl}$ and glomerular filtration $=40 \pm 15.36 \mathrm{ml} /$ min.

The mean dose of cinacalcet increased by $39 \%$ from the start of treatment to the end of the study.

The mean values of PTH at 12 months decreased by $19 \%$ compared to the initial value $(216.4 \pm 144.2 \mathrm{pg} / \mathrm{ml})$ and $32 \%$ at 24 months $(181.1 \pm 55.2 \mathrm{pg} / \mathrm{ml}, p<0.001)$. Regarding the average values of calcium, they were also reduced by $11 \%(9.3 \pm 0.8 \mathrm{mg} / \mathrm{dl})$ at 12 months compared to the initial value, remaining stable at 24 months $(9.6 \pm 0.6 \mathrm{mg} / \mathrm{dl})$. The phosphate values remained stable during the study $(3.3 \pm 0.7 \mathrm{mg} / \mathrm{dl}$ at 24 months $)$.

10 patients presented adverse effects to cinacalcet. All of them were described in the technical data sheet: $50 \%$ hypocalcemia, $20 \%$ myalgia, $20 \%$ asthenia and $10 \%$ nausea and vomiting.

Conclusion: In our study, cinacalcet was effective for the treatment of patients with hyperparathyroidism secondary to chronic renal failure without hemodialysis, significantly reducing the PTH and calcium values.

Disclosure of Interest: None Declared.

HP-PC035: Hospital at home, an alternative to hospitalization: truly in practice? Example of imipenem

C. Ratsimbazafy ${ }^{1, *}$, B. Mittaine-Marzac ${ }^{1}$, O. Marquestaut ${ }^{2}$, L. Havard ${ }^{1}$

${ }^{1}$ Hospital Pharmacy, ${ }^{2}$ Department of Medical Information, Hospitalisation A Domicile - Assistance Publique Hopitaux De Paris (APHP), Paris, France

Background and objective: Hospital-at-home (HAH) is generally defined as a substitutive care model to hospitalization $(\mathrm{H})$. The study investigated if HAH did not merely on paper but was truly implemented in practice, by taking for example patients with imipenem/cilastatine (IMP) infusion in order to define the patients' type treated in HAH.

Design: All patients (adults, infants) in the 820 places of HAH of Assistance Publique Hopitaux de Paris (APHP), covering Paris and its suburb, treated with IMP - an antibiotic drug for complicated infections, restricted to hospital infusion-were analysed retrospectively from 1st/04/2017 to 31st/12/2017. Social, medical, and pharmaceutical items, as well as cost data were collected from patients' records and billed medical charges.

Results: 26 patients (males $=65 \%, 64$ years old [17; 97], Karnofsky score $=45 \%[35 ; 55])$ were treated during 27 home stays, with a mean length of stay (MLS) of 26 days [4; 88]. 25 patients had a helping person and one was isolated. 24 patients were admitted after discharged on average beyond 10 days of antibiotic treatment in $\mathrm{H}$, mostly from surgery and onco-haematology wards. Antibiotic indications were sepsis or urinary tract, bronchopulmonary, and osteoarticular infections. 50\% of patients carried multi-drug-resistant (MDR) bacteria. The treatment start date and treatment duration were respectively known in 15 patients, and in 8 . 
Patients were addressed to HAH for $85 \%$ intravenous infusion, $7 \%$ palliative care, $4 \%$ pain, $4 \%$ complex care of bandages. During the stay at home, 4 patients died, 12 readmissions including 6 unscheduled occurred.

Hospital cost data were collected for 20 out of the 24 patients from $\mathrm{H}$. Average daily costs of care were $633 €$ in $\mathrm{H}$ (MLS $=19.5$ days) against $280 €$ in HAH (MLS $=23.9$ days).

Over the study period, HAH ordered 2064 units of IMP of $500 \mathrm{mg}$, which represents $1.8 \%$ of the consumption of all 39 APHP hospitals. Conclusion: Despite the small size of sample, these results suggest traditional hospital care still remain "the gold standard" for the delivery of antibiotic treatment, in spite of the feasibility and the lower costs of HAH care. In practice, HAH appears to cover patients with specific clinical components: few in number, elderly, dependant patients, often carrying a MDR bacteria, after a long-term hospital stay.

Additionally, the study also revealed further progress was still necessary to improve the fight against antimicrobial resistance, such as medical and nurse staff providing valuable information needed for antibiotic treatment.

Disclosure of Interest: None Declared.

\section{HP-PC036: The reliability and validity study of Turkish Version} of High Alert Medications Knowledge Questionnaire

\section{B. Okuyan', O. Ozkan', B. Torun', F. Ozturk ${ }^{1}$, M. Sancar ${ }^{1, *}$ \\ ${ }^{1}$ Clinical Pharmacy, Marmara University Faculty of Pharmacy, Istanbul, Turkey}

Background and objective: It is aim to assess the reliability and validity of Turkish version of high-alert medications knowledge questionnaire $^{1}$.

Setting and method: This study was conducted between December 2017 and January 2018 among healthcare professionals (18 years or older) working in a private hospital. The adapted questionnaire consisted 20 true-false knowledge items and the higher score was indicated better knowledge regarding high alert medications. After its appropriate language translation and cultural adaptation, the internal consistency by using Kuder-Richardson coefficient and test-retest reliability of the Turkish version of this questionnaire were evaluated. Main outcome measures: Reliability and validity parameters of Knowledge Questionnaire.

Results: Of the 159 individuals, $91.8 \%$ (146) participated to the study and $24 \%$ were males. $69.9 \%$ of the participants were nurses, $2 \%$ were pharmacists and $28.1 \%$ were technicians. The mean age of the participants was calculated as $25.40 \pm 5.16$ (19-56). Test-retest reliability was confirmed $(0.527 ; p<0.01)$ and internal consistency reliability that was assessed by using Kuder-Richardson 20, was acceptable (Cronbach's $\alpha=0.815$ ). Of them, $65.1 \%$ agreed to have 'sufficient' knowledge and $63.7 \%$ hope to acquire more training about high-alert medication. In addition, there is a statistically significant correlation between the number of participants who agreed to have 'sufficient' knowledge about high-alert medication and their total knowledge scores $(p<0.01)$. Advanced age and increased in professional experience were statistically correlated with higher knowledge regarding high alert medications $(p<0.001)$.

Conclusion: As a result of this study, it was determined that the Turkish version of the questionnaire is valid and reliable scale to assess health-care professionals' knowledge towards high-alert medications.

${ }^{1}$ Hsaio GY, Chen IJ, Yu S, Wei IL, Fang YY, Tang FI. Nurses' knowledge of high-alert medications: instrument development and validation. J Adv Nurs. 2010;66(1):177-190).

Disclosure of Interest: None Declared.
HP-PC037: Reason for therapeutic discontinuation in multiple sclerosis patients-experience in an university hospital

\section{O. Simona ${ }^{1, *}$, S. Negres ${ }^{2}$}

${ }^{1}$ Pharmacy Department, Bucharest University Emergency Hospital, ${ }^{2}$ Pharmacology and Clinical Pharmacy Department, University of Medicine and Pharmacy "Carol Davila" Bucharest, Bucharest, Romania

Background and objective: When a patient with Multiple Sclerosis (MS) cannot tolerate disease modifying therapy (DMT), or there is unacceptable breakthrough disease activity, the most common approach is to change drug. All have side effects, and none are cure. No universal guidelines exist for switching therapy and choices of clinicians on this matter are often based on observational reports and guided by good clinical practice.

The variety of DMT available in our hospital (5 different first-line therapies and only one for second-line), allows treatment switch, based on reccomendation.

Objective is assessing safety, switch causes for each DMT, which drugs are less likely to follow discontinuation.

Setting and method: Retrospective observational study was conducted from January 2017 to March 2018, including patients with the following DMT:

Interferon like:

- interferon beta1-a and beta1-b,

- glatiramer acetate (GA),

- natalizumab,

- and teriflunomide,

Main outcome measures: Variables collected: average age at diagnosis, sex, previous treatment, reason for changing treatment, EDSS, presence of antibodies against $\mathrm{JC}$ virus, adverse drug reactions, mean duration of disease. The data from the patient monitoring file reffers to: clinical examination every 3 months, paraclinical investigation:brain magnetic resonance imaging (MRI) — every year and laboratory investigation.

Results: 149 patients with a median exposure to medication 8.83 years (1-19 years), 97 women, mean age at diagnostic 32 (14-63 years), mean baseline EDSS $=1.97(0-6)$.

During the follow-up period, no changes were observed in treatment in more than half of the patients $(53.5 \%)$.

Patients that switched treatment (46.5\%): $39.5 \%$ once, $7 \%$, at least twice and despite treatment, 11 patients $(16 \%)$ evolve to a progressive form-SMSP with relapses and/or active MRI.

Glatiramer was the least likely to follow discontinuation.

3 patients had temporary interruption during pregnancy.

The main reason for changing DMT was lack of efficacy, other reasons were injection-site reactions, persistent flu-like symptoms, clinical activity and only one patient had safety issues- high-risk for PML, with anti-JCV index above 1.5 and long natalizumab exposure (5 years) was withdrawn, switched to glatiramer after a short "drug holiday".

Conclusion: The overall goal of switching MS therapies is to balance the risks posed by the medications to the risks posed by the disease, though prognosticating long-term outcomes in MS is difficult.

Individual response to DMTs varies significantly across patients (within and between subjects) and the risk of serious adverse events represent an issue, particularly for the newest agents, so the main challenge facing clinicians and patients remains choosing a course of personalized treatment, considering all factors.

Disclosure of Interest: None Declared. 
CP-PC016: Pharmacist prescribing and care improves cardiovascular risk, but what do patients think? A sub-study of the RxEACH study

Y. N. Al Hamarneh ${ }^{1, *}$, S. lamb ${ }^{1}$, M. Donald ${ }^{2}$, K. King-Shier ${ }^{2}$, C. Jones ${ }^{3}$, B. Hemmelgarn ${ }^{2}$, C. Mitchell ${ }^{4}$, R. Tsuyuki ${ }^{1}$

${ }^{1}$ University Of Alberta, Edmonton, ${ }^{2}$ University of Calgary, Calgary, ${ }^{3}$ University of British Columbia, Kelowna, ${ }^{4}$ Patient Partner, Grand Prairie, Canada

Background and objective: The Alberta Vascular Risk Reduction Community Pharmacy Project: $\mathrm{R}_{\mathrm{x}} \mathrm{EACH}$, was a randomized trial which demonstrated that a community pharmacy-based case finding and intervention program (including prescribing, laboratory testing, and follow-up) reduced the risk for cardiovascular (CV) events by $21 \%$ when compared to usual care.

Objective: To evaluate patient perceptions regarding pharmacist prescribing and care in patients at high risk for $\mathrm{CV}$ events.

Setting and method: All participants who took part in $\mathrm{R}_{\mathrm{x}} \mathrm{EACH}$ received an invitation letter. Those who took part in the interviews provided verbal consent.

Participants were asked to provide their opinions about:

- The care they received from pharmacists.

- Communication between patients, pharmacists and family physicians.

- Suggestions for sustainability.

Interviews were recorded and transcribed verbatim. Three reviewers (including one patient who did not participate in $\mathrm{R}_{\mathrm{x}} \mathrm{EACH}$ ) analyzed and coded the data independently.

Main outcome measures: Patient perceptions regarding pharmacist prescribing and care in patients at high risk for CV events.

Results: Data saturation was achieved after interviewing 14 participants. Half of whom were male and approximately two-thirds were older than 60 .

The following themes were identified:

(i) Patient-pharmacist relationship: Participants highlighted the importance of having a strong relationship with the pharmacist, indicating that could enhance their level of comfort with the pharmacist. They also felt that pharmacists truly cared about them as people.

(ii) Healthcare system characteristics: The majority of the participants supported expanded scope of practice and identified it as an opportunity to fill healthcare gaps highlighting easy accessibility, high quality and timeliness of pharmacist services.

(iii) Patient reaction: Participants were extremely satisfied with the care they received and reported that they felt empowered when pharmacists encouraged them to take responsibility for their own health

Conclusion: Patients are highly supportive of an advanced scope of pharmacy practice which includes prescribing, follow-up, and remunerated care. Our inclusion of a patient in the analyses provided a unique perspective.

Disclosure of Interest: None Declared.

CP-PC017: Implementing online pharmaceutical care services based on web-based pharmacy care setting: a retrospective pilot study

\section{A. H. Al-Taie ${ }^{1}$, Z. K. Yilmaz,**}

${ }^{1}$ Pharmacy Department, Osol Aldeen University College, Baghdad, Iraq, ${ }^{2}$ Clinical Pharmacy Department, Faculty of Pharmacy-Marmara University, Istanbul, Turkey
Background and objective: Pharmaceutical care services (PCSs) are extremely valuable for patients related outcomes. Implementation of web-based pharmacy care and thereby different online PCSs could have the potential to promote better access to information by patients and providers, improve the quality and safety of patients and encourage healthier lifestyles. The aim of this study was to exhibit the esential role of the pharmacist through the provision of different PCSs that could be conducted via an online platform as a new modernized pharmacy practice.

Setting and method: A retrospective pilot observational study was carried out on patients during their website visiting to İlaçpedia ( https://www.ilacpedia.com/) seeking pharmacists with more in-depth health and medication knowledge regarding different medical conditions. This website is considered as an online drug information and patient counselling service platform regarding healthcare topics, discussions and medication care services in Turkey. Suitable customized online PCSs were provided to patients who considering İlaçpedia for health and medication consultancy and detailed information regarding patient's demographic, health and medication status were collected.

Main outcome measures: Impact of pharmacist care intervention via web-based pharmaceutical care setting offered through different trends of online PCSs including disease-related trends (DRTs) and medication-related trends (MRTs).

Results: A total of 57 participants were included with a mean age of 33.70 years constituting $27(47.4 \%)$ males and 30(52.6\%) females. $29(50.9 \%)$ patients were cigarette smokers. A total of 83 medical conditions encountered with endocrine conditions ( $n=23,27.7 \%$ ) followed by CNS conditions $(\mathrm{n}=20,24 \%)$, dermatologic disorders $(\mathrm{n}=14,16.9 \%)$ and cardiovascular conditions $(\mathrm{n}=11,13.3 \%)$ were the most common medical conditions observed. Among these, acne $(\mathrm{n}=11)$, hypertension $(\mathrm{n}=8)$, anxiety disorders $(\mathrm{n}=7)$ and DM $(n=6)$ were the most reported diseases. Number of medications being used by the participants were (130) medicines representing $(n=120)$ orally administered and $(n=8)$ topically administered medicines alongside $(n=7)$ supplementary medications. Regarding adverse drug reactions (ADRs) patients reported to pharmacist was (no. of ADRs $=36$; no. of suffering patients: $\mathrm{n}=27 ; 47.4 \%$ ). Among the most common ADRs observed was those related to CNS $(\mathrm{n}=13$, $36.1 \%)$ and skin $(\mathrm{n}=10,27.8 \%)$. Different many trends of PCSs were provided to patients $(n=348)$ including DRTs [disease condition and general health information: $\mathrm{n}=57,16.4 \%$; smoking habits control: $\mathrm{n}=27,7.8 \%$ ] and MRTs [proper medication information: $\mathrm{n}=55,15.8 \%$; proper dosage selection: $\mathrm{n}=57,16.4 \%$;proper treatment duration: $\mathrm{n}=56,16.1 \%$;refilling of prescription: $\mathrm{n}=45$, $12.9 \%$;presence of medication side effects: $\mathrm{n}=25,7.2 \%$ and proper drug alternative: $\mathrm{n}=7,2 \%$ ].

Conclusion: The results of this study clearly support the pivotal and effective role of the community pharmacist as a patient-centred healthcare provider and the intriguing trend of web-based pharmacy care for the provision of different PCSs as another mode of community pharmacy practice.

Disclosure of Interest: None Declared.

CP-PC018: Patterns of patients' reliance of online health and pharmaceutical care services as an alternative implemented platform of pharmacy practice

\section{A. H. Al-Taie ${ }^{1}$, Z. K. Yilmaz ${ }^{2, *}$}

${ }^{1}$ Pharmacy Department, Osol Aldeen University College, Baghdad, Iraq, ${ }^{2}$ Clinical Pharmacy Department, Faculty of Pharmacy-Marmara University, Istanbul, Turkey

Background and objective: The increasing prevalence of diseases is proportionally correlated with the growing body of health services 
that could successfully redesign care systems to meet the patients' needs. Different models of healthcare services could be performed by pharmacists that would match pharmacy practice to provide more advanced disease management and medication care services. The aim of this study was to assess the trends of patients' knowledge of online pharmaceutical care (PC) setting alongside types of health and medication care services introduced by the community pharmacists based upon online PC platform.

Setting and method: A retrospective pilot observational study was carried out on patients during their website visiting to İlaçpedia ( https://www.ilacpedia.com/) seeking pharmacists with more in-depth health and medication knowledge regarding different medical conditions. This website is considered as an online drug information and patient counselling service platform that provides patients with comprehensive information regarding healthcare topics, discussions and medication care services in Turkey. Suitable customized online PCSs were provided to patients who considering İlaçpedia for health and medication consultancy and detailed informationfor patient's demographic, health and medication status were collected.

Main outcome measures: Trends of patients' knowledge of online PC and types of health and pharmaceutical care services offered by the community pharmacist via online services.

Results: A total of 57 participants were included with a mean age of 33.70 years constituting 27(47.4\%) males and 30 (52.6\%) females. $29(50.9 \%)$ patients were cigarette smokers. Regarding educational level, majority of participants visiting the website had well education level $(53,93 \%)$ presenting as $21(36.8 \%)$ and $32(56.1 \%)$ patients having secondary and university education level respectively. Regarding the reaching mode of this online pharmacy service, $43(75.4 \%)$ patients had a recognition via own self-seeking followed by $8(14 \%)$ via friends and/or relatives. Types of health and pharmacy care services relied upon issues related to medications, health, and lifestyle (a total of 96 services were provided) including medicationrelated issues $(\mathrm{n}=57,59.4 \%)$ followed by health-related issues $(\mathrm{n}=21,21.9 \%)$ and lifestyle-related issues $(\mathrm{n}=18,18.7 \%)$. The number of times participants visiting this online pharmacy service were in a step up ordering [one time $=25(44 \%) ; 2-3$ times $=$ $20(35 \%) ; \quad 4-5 \quad$ times $=5(8.7 \%) ; \quad 6-10 \quad$ times $=3(5.3 \%) ;>10$ times $=4(7 \%)]$. A total of 83 medical conditions were encountered with endocrine conditions ( $\mathrm{n}=23,27.7 \%$ ) followed by CNS conditions $(n=20,24 \%)$, dermatologic disorders $(n=14,16.9 \%)$ and cardiovascular conditions $(n=11,13.3 \%)$ were the most common medical conditions observed.

Conclusion: The above findings provide compelling evidence that patients particularly those with well education level had a high, intensive assessing and reliability of online pharmaceutical care services as another phase of community pharmacy care setting to help manage different health and medication care concerns.

Disclosure of Interest: None Declared.

CP-PC019: Assessment of attitudes and practice of Turkish community pharmacy staff regarding skin problems using virtual patient profiles

\section{S. Apikoglu-Rabus ${ }^{1, *}$, I. Kahya ${ }^{2}$, M. Serin ${ }^{2}$, P. Yildiz ${ }^{2}$ \\ ${ }^{1}$ Clinical Pharmacy Department, ${ }^{2}$ year-5 Student, Marmara University Faculty Of Pharmacy, Istanbul, Turkey}

Background and objective: Skin problems are commonly encountered in community pharmacies. A considerable amount of patients come to the pharmacies with symptom-related requests for advice. In this study we aimed to assess Turkish pharmacy staff's self-reported counseling practices and attitudes regarding skin problems using virtual patient profiles.
Setting and method: The study was conducted at 100 community pharmacies located in Istanbul. Pharmacy staff that agreed to participate in the study completed a questionnaire, which was structured to elicit their demography, professional experience, counseling practices and attitudes regarding skin problems. This study utilized paper-based virtual patient profiles representing 5 different skin problems: dermatitis, athlete's foot, diaper rash, acne and psoriasis. Each staff was requested to act as if they were counseling with the patients identified in three virtual patient profiles. Brief information about and a high-resolution photo of the condition were provided to the staff. More information about the virtual patient was only provided if the staff asked for it by specific questions. Attitudes were measured by questions with 5-Likert answer choices as: "totally agree, agree, neither agree nor disagree, disagree and totally disagree".

Main outcome measures: The rate of counseling on various aspects of the skin problems and the rate of correct attitudes.

Results: The study was conducted on 100 pharmacy staff (76 pharmacists and 24 pharmacy technicians); $53 \%$ was male and the mean age was 42.72 (12.57). They have been practicing at the community pharmacy for 17.65 (10.93) years and serving a median (min-max) of 30 (3-300) patients/week with skin problems. The pharmacy staff completely agreed or agreed that the reason of the patients' visiting the pharmacy in order to receive advice for their skin problems were "high availability of pharmacy staff $(97 \%)$, familiarity with pharmacy staff $(97 \%)$, advice with no cost $(95 \%)$, triage $(89 \%)$, reassurance $(84 \%)$. On the other hand only $23 \%$ of pharmacy staff completely agreed or agreed that "they lack dermatologic knowledge". When the counseling practices of the pharmacy staff were assessed, it was seen that $41 \%$ of the pharmacy staff asked for detailed information from the patient and $79 \%$ defined the disease/condition correctly, while only $40 \%$ recommended the correct product and $22 \%$ provided correct information on how to use the product. When the overall approach to the cases were assessed it was seen that only $23 \%$ of the pharmacy staff followed the most correct way (i.e. "Takes detailed patient history, defines the condition, recommends a product, provides patient education" or "takes detailed patient history, defines the condition, refers to the physician" depending on the case).

Conclusion: Our results showed that although the pharmacy staff think that they have enough dermatology knowledge, they were only able to show their knowledge while identifying the problem; they need to receive more education on product selection and product information.

Disclosure of Interest: None Declared.

CP-PC020: Opinions of the Syrian patients about the effect of language barriers on the quality of pharmacy services received and about the use of pictograms

S. Apikoglu-Rabus ${ }^{1, *}$, R. Barri ${ }^{1}$, A. Alsaleh ${ }^{1}$, R. Muhammad Umar ${ }^{2}$

${ }^{1}$ Clinical Pharmacy Department, Marmara University Faculty of Pharmacy, ${ }^{2}$ Clinical Pharmacy Department, Istanbul Medipol University Faculty Of Pharmacy, Istanbul, Turkey

Background and objective: Since the beginning of the Syrian conflict, over 3,5 million Syrians have fled their homes and been living in Turkey. Although, the government covers all health care costs including the medications of the refugees, lack of language skills is a major problem limiting the utilization of quality health care both at hospitals and at community pharmacies. This study aimed to investigate the opinions of the Syrian patients about the effect of language barriers on the quality of pharmacy services received at community pharmacies and about the usefulness of pictograms. 
Setting and method: The study was conducted at three community pharmacies where medications of the Syrian refugees were provided according to a contract. All Syrian patients who visited these pharmacies during a week's time were informed about the study and invited to participate. All participating patients were asked to fill in an Arabic questionnaire asking for demographic data and opinion about the effect of language barriers on the quality of health-care and pharmacy services. An example of pictogram use (i.e. Voltaren retard ${ }^{\circledR}$; with five pictograms as 'for back pain; to be taken in the morning; to be taken on full stomach, to be taken one tablet at once; should not be crushed, broken and chewed') was shown to the patients and their opinion about the usefulness of the pictogram was questioned.

Main outcome measures: Opinions of the participants about the effect of language barriers on the quality of pharmacy services and about the usefulness of pictograms.

Results: Fifty-three patients [mean (SD) age $=32(13.15)$ years; $78 \%$ male] participated in the study. Vast majority (93\%) of the participants were refugees and they have been living in Turkey for 3.58 (1.16) years; $28 \%$ were primary-school graduates and $34 \%$ were secondary school graduates; $44.4 \%$ were working. Forty-seven percent of the patients totally agreed or agreed that incompetency in Turkish language skills negatively affect the pharmacy services they receive from community pharmacies. More than half of the patients totally agreed or agreed that they receive enough information about the correct use of their medications (69\%) and warnings and side effects of their medications $(61 \%)$. Majority $(80 \%)$ totally agreed or agreed that they would have used their medications more correctly if they had received instructions by the use of pictograms. In general $(91 \%)$ they reported that they were satisfied by services they received from the community pharmacies.

Conclusion: The language problem seems to be a barrier for the Syrian patients to receive the best quality pharmacy services. Use of pictograms while instructing on medication use would help the nonnative speakers receive the most relevant information and result in the correct use of the medications.

Disclosure of Interest: None Declared.

CP-PC022: The experience of people with type 2 diabetes being cared for by their community pharmacist in Ireland

\section{P. Cooney ${ }^{1}$, J. Hanley ${ }^{1}$, N. Ryan-O'Brien ${ }^{1}$, H. Okada ${ }^{2}$, M. Bermingham,}

${ }^{1}$ School of Pharmacy, University College Cork, Cork, Ireland,

${ }^{2}$ EPICORE Centre, University of Alberta, Edmonton, Canada

Background and objective: The Future Pharmacy Practice in Ireland report recommends expanded pharmacy-based services for people with type 2 diabetes. ${ }^{1}$ These services may include structured patient education, adherence monitoring, medication use review and supplementary prescribing. The aim of this study is to evaluate the experience of people with type 2 diabetes being cared for by their community pharmacist in Ireland, which may inform expansion of pharmacy services to this patient group.

Setting and method: Questionnaire (adapted from Stewart et al. ${ }^{2}$ ) was administered in seven Irish community pharmacies. Participants were type 2 diabetics, aged $\geq 18$ years. Participants were asked five "yes or no" questions about their medications and then scored 13 statements on a five-point Likert scale (strongly disagree $=1$ to strongly agree $=5$ )

Main outcome measures: Likert scale responses for each statement were summarised as frequencies and percentages. The mean $( \pm \mathrm{s}-$ tandard deviation) score for each item was calculated.

Results: Data were available for 125 participants, average age $65.7 \pm 12.4$ years and $74(59.2 \%)$ were male. Oral hypoglycaemic therapy was prescribed to $123(98.4 \%)$ participants, parenteral hypoglycaemic agents to $15(12.0 \%)$ participants and insulin products to $20(16.0 \%)$ participants. The statement that participants most frequently agreed with was "I am totally satisfied with my visit to this pharmacist" (agree/strongly agree $=99.2 \%$, mean score $4.9 \pm 0.4$ ). There was also strong agreement with the statement "I would recommend seeing a pharmacist to other people" (agree/strongly agree $=95.2 \%$, mean score $4.7 \pm 0.7$ ). A high proportion of participants $(81.6 \%)$ agreed/strongly agreed with the statement "It is easier to get to see the pharmacist than the doctor" (mean score $4.3 \pm 1.1$ ); $19.2 \%$ of patients disagreed with the statement "I am more comfortable discussing medication-related issues with the pharmacist than my doctor" (mean score $3.5 \pm 1.2$ ) and $85 \%$ of participants disagreed or strongly disagreed with the statement "Some things about my consultation with the pharmacist could have been better" (mean score $1.7 \pm 1.1)$.

Conclusion: In this Irish community-based cohort, participants with type 2 diabetes reported a high level of satisfaction with the care provided to them by their pharmacist. This augurs well for implementation of expanded community pharmacy services for this patient group.

\section{References}

1. Pharmaceutical Society of Ireland. Future Pharmacy Practice in Ireland: Meeting Patients' Needs Report 2016.

2. Stewart D et al. Developing and validating a tool for assessment of pharmacist prescribers' consultations. Fam Pract. 2010;27(5):520-526.

Disclosure of Interest: None Declared.

CP-PC023: Community Pharmacy Scotland: the Minor Ailment Service as perceived and experienced by patients: a pilot study

L. Boag ${ }^{1, *}$, A. C. Boyter ${ }^{2}$, K. MacLure ${ }^{3}$, S. Cunningham ${ }^{3}$, G. Akram ${ }^{2}$, H. McQuillan ${ }^{4}$, D. Stewart ${ }^{3}$

${ }^{1}$ Robert Gordon University/University of Strathclyde, Aberdeen, ${ }^{2}$ University of Strathclyde, Glasgow, ${ }^{3}$ Robert Gordon University, Aberdeen, ${ }^{4}$ Community Pharmacy Scotland, Edinburgh, United Kingdom

Background and objective: The Minor Ailment Service (MAS) in Scotland has provided free treatment via community pharmacy, for those eligible, for self-limiting conditions since its inception in 2006. MAS is a source of free expert advice and treatment which is readily available with no need for an appointment. This service contributes to the national enhancement of healthcare: improving access to services and minimising health inequalities. In 2016/17 over 2 million overthe-counter products were prescribed by pharmacists on MAS costing $£ 4.9$ Million. However, there is no information on the experiences of service users at a national level. A national evaluation is therefore planned in the latter part of 2018. The aim of this study is to gauge project feasibility, test the planned data collection and handling of the full scale project, and to obtain an expected response rate.

Setting and method: Five community pharmacies across Scotland were selected for the pilot study on the basis of deprivation and locations. Each pharmacy was provided with 25 study packs containing an initial questionnaire. Those service users who returned the initial questionnaire were mailed a follow-up questionnaire.

Main outcome measures: The initial questionnaire included items on: previous use of MAS, presenting condition, expectations, reasons for using MAS, perceived privacy, planned use of other healthcare service(s), perceptions of consultation and views of pharmacist care (Consultation and Relational Empathy: CARE) and self-reported quality of life. The follow up questionnaire determined: perceived 
effectiveness of treatment, other sources of treatment, further use of MAS and self-reported quality of life.

Results: Of the possible 125 questionnaires a total of 52 were distributed in a 1 week period. Pharmacies in the least deprived areas distributed the fewest study packs $(\mathrm{n}=11)$, while the most deprived areas distributed the most $(\mathrm{n}=41)$. Of the 52 questionnaires distributed 14 were returned completed: a response rate of $27 \%$. The pilot responses informed questionnaire development with minor changes in layout to increase response rate, and reduction in open ended questions which were rarely completed. Responses from the pilot questionnaires provide positive indications regarding MAS experiences but lack the statistical power to provide representational results. The pilot study has however provided the framework for the full scale study.

Conclusion: The full study will distribute 10 study packs from every community pharmacy in Scotland within a 2 weeks period. Given the number distributed in the pilot, this should be achievable. Using the pilot response rate the full project should return in excess of 3000 questionnaires. The full study will provide national patient experience of MAS, allow quantification of the transfer of care to community pharmacy from other healthcare services and service users perception of effectiveness.

Disclosure of Interest: None Declared.

\section{CP-PC024: Services provided by pharmacists in Swiss nursing homes}

L. D. Brühwiler ${ }^{1, *}$, A. Niederhauser ${ }^{1}$, L. Fishman ${ }^{1}$, D. L. Schwappach ${ }^{1,2}$

${ }^{1}$ Patient Safety Switzerland, Zürich, ${ }^{2}$ Institute of Social and Preventive Medicine (ISPM), University of Bern, Bern, Switzerland

Background and objective: Drug-related problems are frequent among nursing home $(\mathrm{NH})$ residents. A designated pharmacist may detect and solve these problems, e.g. by reviewing residents' medications. The collaboration with a pharmacist in Swiss NH may however, due to heterogeneous regional regulations, vary widely. Therefore, we sought to analyse how often NH collaborate with a designated pharmacist and how widespread services e.g. medication reviews by pharmacists are. In addition, we aimed to examine in what types of NH medication reviews by pharmacists could further be disseminated.

Setting and method: Online survey to directors of nursing in all Swiss NH with $\geq 10$ beds $(\mathrm{n}=1525)$. $\mathrm{Chi}^{2}$-test was used to examine the association between categorical variables.

Main outcome measures: Percentage of $\mathrm{NH}$ with a designated pharmacist (NH-P), frequencies and types of pharmaceutical services in NH-P, characteristics of $\mathrm{NH}$ where pharmacists don't provide medication reviews.

Results: $420(27.5 \%)$ questionnaires were returned. $81.8 \%$ of $\mathrm{NH}$ collaborate with a designated pharmacist (329 NH-P among $402 \mathrm{NH}$ ). Pharmacists provide an array of different services, but with variable dissemination. Medication reviews by a pharmacist are provided in $40.7 \%$ of NH-P. In those NH-P, self-reported satisfaction with medication review processes was higher $(79.1 \%$ satisfied) than in NH-P where pharmacists don't provide medication reviews (59.0\% satisfied, $p<0.001)$.

We found that medication reviews by pharmacists were less frequently provided in $\mathrm{NH}$ mainly collaborating with independent general practitioners $(30.3 \%)$ than in $\mathrm{NH}$ mainly collaborating with employed physicians $(45.8 \%, p<0.05)$. Furthermore, there was an association with the NH's region: Pharmaceutical reviews were less frequent in regions where self-dispensing of medications by physicians is permitted, i.e. most German-speaking regions (23.8\%) than in regions where this is prohibited $(49.3 \%, p<0.001)$. There was no association between the provision of medication reviews and number of beds, total number of physicians per $\mathrm{NH}$ and organisation type.

Conclusions: A majority of Swiss nursing homes collaborate with a designated pharmacist, but provided services by pharmacists vary. $\mathrm{NH}$ in the German-speaking part of Switzerland who collaborate with general practitioners could be targeted to disseminate pharmacist involvement in medication reviews in order to improve medication safety.

Disclosure of Interest: None Declared.

CP-PC025: Pharmaceutical care for the institutionalized patient in nursing homes

C. Campabadal ${ }^{1, *}$, F. Bejarano', N. Marco², L. Palacios ${ }^{3}$, D. Ferrer-Vidal ${ }^{4}$, L. Canadell ${ }^{1}$

${ }^{1}$ Pharmacist, ${ }^{2}$ Administrator, ${ }^{3}$ Care Director, ${ }^{4}$ Director, Hospital Joan Xxiii Tarragona, Tarragona, Spain

Background and objective: Guarantee comprehensive and quality pharmaceutical care for institutionalized patients in nursing homes $(\mathrm{NH})$ based on equity, safety, efficiency and effectiveness.

Design: The pharmacist evaluates the pharmacotherapeutic plans (PTP) of all residents. The review consists of an evaluation of the indication, effectiveness, safety, Stopp-Start criteria, drug based on evidence, follow-up of the pharmacotherapeutic guide, efficiency, dose correction and guidelines. Once the evaluation is completed, the Pharmacotherapeutic Advisory Commission (CAP), created by the management and formed by the doctors and nurses of the $\mathrm{NH}$ and the team and the pharmacist, meets. The CAP evaluates in a multidisciplinary way the pharmacotherapeutic plans and consensual changes in medication. Once the medication changes are agreed upon, they are proposed to the patient or their relatives. The changes are applied in the different computer programs.

Results: In the 6 months of the project this has been implemented in 8 of the $26 \mathrm{NH}$ of the territory. 402 PTPs have been reviewed, representing $22 \%$ of patients. Some incidence has been found in $85.8 \%$. A total of 697 drugs were reviewed, of which $47.02 \%$ had an incorrect indication and $30 \%$ required clinical assessment by the CAP. Of the incidences present in the PTP, $86.3 \%$ was an innecessay drug, followed by a drug not recommended in geriatrics $(4.17 \%)$, duplicity $(2.36 \%)$, patient with 2 or more benzodiazepines $(0.34 \%)$ and others. Of the proposals made by the pharmacist the majority was to suspend the drug $(84.12 \%)$, change by therapeutic equivalent $(9.75 \%)$, decrease dose $(4.74 \%)$, start a treatment $(0.84 \%)$ and others. $94 \%$ of the proposals made by the pharmacist have been accepted. Of the $6 \%$ of the proposals not accepted, $60 \%$ is due to the $\mathrm{NH}$ doctor, $30 \%$ to the patient/family member and $6 \%$ to the team doctor. An annual theoretical saving of $€ 79,813$ is estimated with $22 \%$ of the patients reviewed. This represents an average decrease of $24.8 \%$ of the annual turnover per residence.

Conclusion: Pharmaceutical care for institutionalized patients in nursing homes contributes significantly to an improvement in the quality of the pharmacotherapy prescription.

Disclosure of Interest: None Declared.

CP-PC026: Pharmaceutical care for patients with renal insufficiency and treatment with oral antidiabetics

C. Campabadal ${ }^{1, *}$, F. Bejarano' ${ }^{1}$, N. Marco $^{2}$, L. Palacios ${ }^{3}$, D. Ferrer-Vidal ${ }^{4}$, L. Canadell ${ }^{1}$

${ }^{1}$ Pharmacist, ${ }^{2}$ Administrator, ${ }^{3}$ Care Director, ${ }^{4}$ Director, Hospital Joan Xxiii Tarragona, Tarragona, Spain 
Background and objective: Provide the safest and most suitable treatment in patients with chronic renal failure and in treatment with oral antidiabetics.

Design: All patients with chronic kidney disease (using CockroftGault formula) who were taking oral antidiabetic drugs were listed. The glomerular filtration and type of drug data were crossed based on the glomerular filtration value from which the drug requires a dose adjustment or is contraindicated, so that only those patients who a priori needed a dose adjustment in their treatment were reviewed.

The review carried out by the Pharmacy consists of the last glycated hemoglobin of the patient with date of analysis, the last glomerular filtration of the patient and the current treatment with oral antidiabetics.

The patients controlled by endocrinology were reviewed jointly with the doctor responsible for the patient.

Personalized reports for each patient were sent to their responsible physician, enclosing recommendations for change in the proposed treatment of Pharmacy.

Results: We reviewed 60 patients with an average age of $77 \pm 9.9$ years, with an average glomerular filtration rate of $43 \pm 13.8 \mathrm{~mL} / \mathrm{min} / 1.73 \mathrm{~m}^{2}$.

The 60 patients had an average of $2 \pm 0.84$ drugs. This entails that a total of 144 treatments have been reviewed.

Of the 144 treatments reviewed, $54(37.5 \%)$ were contraindicated due to the glomerular filtration of the patient and $14(9.72 \%)$ required a dose adjustment, the rest of the treatments were indicated based on glomerular filtrated.

16 patients were commented toghether with endocrinology service.

Of the 60 emails sent, in 20 (33.3\%) patients the recommendations issued by pharmacy have been followed, $4(6.66 \%)$ patients have a normalized renal function that does not need dose adjustment of the antidabetic treatment and $36(60 \%)$ patients are waiting to make the changes.

This revision is made 20 days after sending the mails.

Conclusion: An exhaustive review of oral anti-diabetic treatments is necessary in patients with chronic renal insufficiency to ensure safety in their treatment.

Disclosure of Interest: None Declared.

\section{CP-PC027: Exploring the potential for improved community pharmacy breastfeeding support-expectations and priorities of breastfeeding parents and community pharmacists}

\section{M. D'Arcy ${ }^{1, *}$, R. Duignan ${ }^{1}$, S. McCarthy ${ }^{2}$, M. C. Henman ${ }^{1}$}

${ }^{1}$ School of Pharmacy and Pharmaceutical Sciences, Trinity College Dublin, Dublin, ${ }^{2}$ School of Pharmacy, University College Cork, Cork, Ireland

Background and objective: Although the World Health Organisation recommends exclusive breastfeeding for the first 6 months with continued breastfeeding up to 2 years of age or beyond, breastfeeding rates in the Republic of Ireland (RoI) are among the lowest recorded. The accessibility and health promotion role of the community pharmacy in the RoI suggest potential for community pharmacy based breastfeeding support. Furthermore, there is potential to better explore the full pharmaceutical care needs of this patient cohort.

Setting and method: This was an academic study separately surveying community pharmacists (CPs) and breastfeeding parents as stakeholders (SHs). A subsection of the survey is presented, based on suggested services relating to (a) pharmaceutical care of the breastfeeding patient (e.g. medication safety, nipple care, mastitis, timing of feeds and medication) and (b) other services potentially available from the community pharmacy (e.g. advice on breast pump selection, breastfeeding support information and the pharmacy environment).
Respondents indicated which advice and services they would (i) currently expect and (ii) ideally (for breastfeeding support) expect from a community pharmacy. For the $\mathrm{CP}$ group, current expectations were determined by whether the services were available in their current community pharmacy workplace, or whether they had recently given advice on listed topics.

Main outcome measures: Description of service expectations in the current vs ideal setting in $\mathrm{CP}$ and $\mathrm{SH}$ cohorts and comparison between cohorts.

Results: $50 \mathrm{CPs}$ and $158 \mathrm{SHs}$ responded to the surveys. The highest proportion of respondents in both cohorts expected advice on medication safety while breastfeeding, in both current and ideal settings. The rank order of expectations was similar for both cohorts, and high ideal expectations not achieved in current practice were evident. A notable difference $(40 \%)$ between current and ideal expectations related to the provision of general information on breastfeeding support and support groups (SH group), and the provision of practical advice around expression, storage and feeding of expressed milk along with identification and treatment of mastitis (CP group). Of interest, the proportion of $\mathrm{SH}$ respondents ideally expecting a comfortable place to breastfeed in the pharmacy which is not necessarily a private space was higher than the $\mathrm{CP}$ group, a greater proportion of whom ideally expected to offer a private space. The promotion of breastfeeding as the norm in all interactions with the breastfeeding mother or their representative was expected by a higher proportion of the SH cohort than the $\mathrm{CP}$ cohort in current (53\% CPs; $73 \% \mathrm{SHs}$ ) and ideal (63\% CPs; $91 \%$ SHs) scenarios.

Conclusion: Expectations are rightly high in both $\mathrm{CP}$ and $\mathrm{SH}$ groups with respect to advice on medicines use while breastfeeding. Priority actions CPs could take to offer a more supportive environment for breastfeeding parents include promotion of breastfeeding as the norm, provision of information on local breastfeeding support and a comfortable area to breastfeed in the pharmacy.

Disclosure of Interest: None Declared.

CP-PC028: A pharmacist-delivered smoking cessation program in Qatar: a qualitative exploration of pharmacists' and patients' experiences and perceptions with the program

M. S. El Hajj, ${ }^{1, *}$ A. Awaisu' ${ }^{1}$, S. A. S. Sheikh Ali ${ }^{1}$, N. Kheir ${ }^{2}$, R. Shami ${ }^{1}$

${ }^{1}$ Qatar University College Of Pharmacy, Doha, Qatar, ${ }^{2}$ The

University of Auckland, Auckland, New Zealand

Background and objective: Tobacco use is one of the major preventable causes of death and diseases in Qatar and globally. There is a great opportunity for pharmacists practicing in Qatar to promote tobacco cessation. An intensive pharmacist-delivered smoking cessation program was designed and implemented in 8 primary care pharmacies in Qatar. This study aimed to qualitatively explore the experiences of pharmacists and patients who were involved in the program, to elucidate their perceptions of the barriers and facilitators to implement the program, and to identify the strategies needed to improve it.

Setting and method: The study used an exploratory qualitative study design that employed semi-structured interviews of a random sample of patients and pharmacists who participated in a pharmacist-delivered smoking cessation program in Doha, Qatar. The interviews with pharmacists were conducted face to face in meeting rooms at Qatar University College of Pharmacy. Due to the difficulty of bringing patients to the university campus, the interviews with patients were conducted over the phone. Interviews were facilitated using a designed peer-reviewed semi-structured interview guide. The interviews were audio-recorded and transcribed verbatim with the use of the recorder's notes. Transcripts were reviewed many times by the 
study team, and a code list was generated based on emerging patterns. A thematic approach of data analysis was used.

Main outcome measures: Experiences of pharmacists and patients who were involved in the program, their perceptions of the barriers and facilitators to implement the program, and their perceived strategies needed to improve it.

Results: Eight pharmacists and 22 patients were interviewed. Five preliminary themes emerged. The first theme described the experiences with the program, including the role of the pharmacists, the program benefit on patients, and the program impact on pharmacists' skills. The second theme addressed the barriers to implementing the program including: lack of time, lack of patient commitment and motivation, and cultural barriers. The third theme identified the facilitators including: peer and management support, organized program structure, the use of tools, and the availability of free medications. The fourth theme outlined the smoking cessation strategies used by the pharmacists including: pharmacologic medications and behavioral modifications, use of role models, and continuous follow-up with patients. The fifth theme included strategies to improve the program including: improving the pharmacist training workshop, expanding the pharmacist-delivered smoking cessation program, improving the program facilities and tools, and increasing the program publicity.

Conclusion: The program was perceived to be beneficial in helping patients quit smoking, and it positively contributed to advancing the role of pharmacists in Qatar. The study findings can guide the development of successful pharmacist-led smoking cessation programs in Qatar.

Disclosure of Interest: None Declared.

\section{CP-PC029: Patients ' experiences with and attitudes} towards generic substitution

\section{A. G. Granas ${ }^{1, *}$ \\ ${ }^{1}$ School of Pharmacy, University of Oslo, Oslo, Norway}

Background and objective: Generic substitution was introduced in Norway in 2001. Generics has been met with skepticism by some patients and health professionals. Pharmacy staff spend a lot of time explaining the implications of generic substitution to patients. In this study, we aim to explore if patients ' information needs were met by physicians and pharmacy staff, and if socio-economic conditions affect the attitudes and experiences of patients.

Setting and method: Randomly selected community pharmacies in Oslo, Norway: one pharmacy from each of the 15 city districts. Five pharmacy students interviewed patients. All pharmacy customers were asked by to participate, and those fulfilling inclusion criteria were included. Data was analyzed on SPSS.

Main outcome measures: Experiences with and attitudes towards generic substitution.

Results: We collected the 534 questionnaire from pharmacy customers. Of these, $74.5 \%$ of patients reported high trust in generic drugs, and $80 \%$ accepted generic substitution when this was offered by pharmacy staff. However, they reported knowledge of generic drugs. Trust in generics was lower for polypharmacy patents. One in four patients reported that changes in the look of the tablet or packaging made it more difficult to manage their medicines. One in three patient reported that changes in the names of medicines made it more difficult to manage their medicines. One in five had experienced a decline in effect and $13 \%$ have experienced new or more side effects. One in six experienced better effect and one in ten had fewer side effects from the generic drug. Patients ' attitudes to generics as more positive if they have received information about generics from their doctor. Respondents with higher level of education was more positive to generic substitution, whereas elderly were more skeptical to change.

Conclusion: Lack of knowledge about generic drugs affects patients' attitudes towards generic substitution. More information from physicians about the generic switch can potentially increase the degree of the acceptance of the patients.

Disclosure of Interest: None Declared.

CP-PC030: Do community pharmacists address sexually transmitted infections during a consultation on emergency hormonal contraception? A focus group discussion

M. Haag ${ }^{1, *}$, N. Wittwer ${ }^{1}$, K. E. Hersberger ${ }^{1}$, I. Arnet ${ }^{1}$

${ }^{1}$ Pharmaceutical Care Research Group, University of Basel, Basel, Switzerland

Background and objective: Since 2002, emergency hormonal contraception (EHC) is dispensed as Pharmacists only medicine in Switzerland. Increased access to EHC did not lead to increased risky sexual behavior of EHC users. Nevertheless, the rate of sexually transmitted infections (STIs) has steadily increased in Western countries. Chlamydia is the most commonly reported STI affecting mainly young people $\leq 25$ years i.e., those who are most likely to need EHC. A tick box in the official Swiss EHC protocol indicates that to inform EHC users about the risk of contracting STIs is optional. We aim to assess factors that influence community pharmacists to address STIs during a consultation on EHC.

Setting and method: Community pharmacists around Basel were invited by phone calls to participate in a focus group discussion if they had experience in dispensing EHC. A set of 8 questions was developed about STI counseling during a EHC consultation. Factors were gathered with open questions, and yes/no voting was used to prioritize them. Strong consensus was defined as identical answers but one $(91.7 \%)$. The discussion was audio recorded and logged by two members of the research team. Verbatim transcription and data analysis were performed using MAXQDA.

Main outcome measures: Factors that trigger STI counseling during EHC consultation with emphasis on chlamydia.

Results: A focus group discussion was performed on the 20th March, 2018 in Basel with 12 community pharmacists (age 26-59 years; experience 2-33 years; 9 women). Participants reported to inform every EHC user about STIs, but especially when they (a) were young (b) would often change sexual partner, or (c) would require EC several times (unanimity). Implicit statements are mostly used such as "You know that...". Attention is not expressly drawn to chlamydia. Conclusion: Community pharmacists regard as their duty to inform EHC users about STIs. However, general statements are enumerated in a cautious way. Whether this strategy contributes to safer sexual behavior and ultimately to containment of STIs needs further investigation.

Disclosure of Interest: None Declared.

\section{CP-PC031: Attitudes of polymedicated patients towards generic} drugs in Switzerland

\section{T. L. Imfeld-Isenegger ${ }^{1, *}$, V. Albert ${ }^{1}$, K. E. Hersberger ${ }^{1}$}

${ }^{1}$ University of Basel, Basel, Switzerland

Background and objective: In Switzerland generic drug substitution is not mandatory. Pharmacists are allowed and encouraged to substitute and they receive a small compensation of lost margin. Generic drug substitution may decrease costs in the health care systems, however the use of generic drugs also poses multiple challenges for 
patients. We aimed to assess the attitudes of polymedicated patients towards their own generic drugs.

Setting and method: Fifth-year pharmacy students of the University of Basel reported a medication review (medication history + patient interview) during their internship in community pharmacies. Patients with $\geq 4$ drugs taken during 3 months, with at least one generic drug and one drug from ATC group C (cardiovascular system) were eligible. In addition, students performed structured interviews using open-ended and closed questions about knowledge on and attitudes towards generic drug substitution.

Main outcome measures: Patients' awareness of generic drugs in their therapy, perception of specific aspects, and attitudes towards drug substitution.

Results: The medication review including the interview was conducted with 136 patients (median age 74 [range 49-91], 58.8\% male). Patients' medication lists comprised 9.94 drugs (range 4-26). More than a quarter $(27.9 \%)$ of the interviewed patients were not aware of having a generic drug in their medication list. Asked about their perceptions (multiple answers possible) $29.1 \%$ of the patients could not attribute any advantage, whereas the price was mentioned as advantage by $67.7 \%$. The majority of the patients $(77.4 \%)$ prefer generics, if they would get a new drug prescribed.

Conclusion: In a setting without mandatory generic substitution, patient counselling on generic drugs is important to improve patient's awareness, to address potential advantages and to achieve improved rates of generic drug use through shared decision making.

Disclosure of Interest: None Declared.

\section{CP-PC032: Measurement of knowledge levels of hypertensive patients}

\section{E. Karatas Kocberber ${ }^{1, *}$, G. Ozen ${ }^{2}$, O. Bilgin ${ }^{3}$,} B. S. Uydes-Dogan'

${ }^{1}$ Clinical Pharmacy Department, ${ }^{2}$ Pharmacology Department, Faculty of Pharmacy, Istanbul University, ${ }^{3}$ Faculty of Pharmacy, Istanbul University, Istanbul, Turkey

Background and objective: The incidence of hypertension increases steadily with the increase in expected life span. The prevalence of hypertension was found to be $33.7 \%$ in a study in Turkey. The lack of a rational treatment plan increases cardiovascular risk and can lead to serious complications. For this reason, it is very important to educate patients with hypertensive disorders, to teach blood pressure measurement correctly and to train self-monitoring.

Setting and method: This prospective study was conducted in a total of 100 hypertensive patients were randomly selected from patients coming to the pharmacy for prescription. Patient information was collected via the questionnaire of rational drug use in the treatment of hypertension. The questionnaire is designed to measure the level of knowledge about patients' treatment of hypertension.

Main outcome measures: The purpose of the study is to measure the knowledge level of hypertensive patients.

Results: $68 \%$ of the participants were female, $32 \%$ were male. Patients were taken to study at different levels of education. Only $28 \%$ of the patients measure the blood pressure correctly at all stages. The question with the most incorrect answer $(60 \%)$ is sitting position in the measurement of hypertension. The most correct answer has given is $(84 \%)$ not to speak during the measurement. $80 \%$ of the patients are satisfied with the education given by the pharmacist. In $20 \%$ of the patients, the reasons why patients are not satisfied with the education are that Pharmacist's information is insufficient $(67.85 \%)$, pharmacist is not in the pharmacy $(14.28 \%)$, the communication skills of pharmacist are insufficient $(10.71 \%)$, the pharmacist is very busy $(7.14 \%)$. There is a significant negative correlation $(p<0.05)$ between the level of education of the patients and the training they have received from Doctors, Pharmacists and Pharmacy technicians. Conclusion: The pharmacist plays an important role in increasing the patient's level of knowledge, increasing treatment adherence, and training in progressive self-monitoring methods, such as blood pressure measurement.

Disclosure of Interest: None Declared.

CP-PC033: Opportunities and challenges with direct-to-consumer genetic tests in personalised care

\section{Y. Kiselev ${ }^{1}$,*, C. Johannessen Landmark ${ }^{1}$, A. Baftiu ${ }^{2}$}

${ }^{1}$ Programme for Pharmacy, OSLOMET - Oslo Metropolitan University, ${ }^{2}$ Norwegian Medicines Agency, Oslo, Norway

Background and objective: Direct-to-consumer genetic tests (GTs) have been gaining global popularity in recent years, while personalisation of care is widely seen as an approach in securing effective and safe treatment. The aim of this literature analysis was to investigate medical and legal challenges with the use of GTs for improved pharmacy care.

Design: A search was performed in PubMed and Google Scholar from January 2010 to March 2018. Peer-reviewed original articles and reviews in English were included. EUR-Lex and Lovdata.no databases were used to identify relevant regulations in EU and Norway. Results: GTs can be purchased at pharmacies or ordered by mail. The former are usually marketed as diagnostic tests for specific conditions like lactose intolerance, indicative towards individual's metabolic or athletic properties. In Norway several GTs were previously available at pharmacies but were unexpectedly retracted for the market. In several EU countries GTs are currently sold at pharmacies, including paternity tests. GTs are also available for ordering online, and usually include information on predisposition to common diseases, carrier status for hereditary conditions, response to medications and geno-geographic origin. The largest provider claims to have more than 3 million customers. Publications suggest that GTs may be a valuable instrument to secure rational pharmacotherapy by early identification of risks for serious adverse drug reactions (ADRs) and by enabling choice of therapies most likely to succeed in a patient with a given genotype. The major technological disadvantage of most GTs is that only panels of SNPs are sequenced, which reduces the costs but limits the coverage. Interpretation of GTs is complicated by the deficient scientific evidence and lack of competence among medical professionals. There is little evidence that taking GTs will actually provide health benefits or safer treatment. Transmission of personal genetic data to private companies is worrisome. Among the EU countries legislation is diverse from virtually banning GTs to fully permitting them. In Norway the sale of GTs is not regulated by law but testing of children is forbidden.

Conclusion: Most published studies deal with validity of GTs, bioethics, and legislation. Major challenges with GTs are deficient scientific evidence, low public and professional literacy in genomic health, and vague regulation of GTs. There is a lack of scientific proof for cost-effectiveness of GTs in relation to health outcomes. GTs may contribute to personalised choice of treatments, reduction of ADRs, early diagnostics, and better health awareness, but progress is necessary in validating GTs and elaborating regulations.

Disclosure of Interest: None Declared.

PC034: Professional services in Norwegian community pharmacies, with focus on a possible new service for patients with migraine

T. Krogstad ${ }^{1, *}$, L. M. Vhile Hanssen ${ }^{2}$ 
${ }^{1}$ Natural Sciences and Health, ${ }^{2}$ Oslo Metropolitan University, Oslo, Norway

Background and objective: The community pharmacies in Norway have been through major changes during the last 10-20 years. The last 2 years the Norwegian Government has approved several professional services that pharmacists, mainly those working in community pharmacies, offer to patients. Examples are guidance of inhalation of patients with asthma or Chronic obstructive pulmonary disease, new medicine service and vaccination.

In this study we wanted to investigate the pharmacists' perception of these established services and a suggested service for patients with migraine.

Setting and method: Ten pharmacists from different pharmacies near Oslo, the capital of Norway, were recruited by phone. They were interviewed personally with a semi-structured questionnaire. All the three major pharmacy chains were represented.

Main outcome measures: To investigate the pharmacists' perception of the established services in Norwegian pharmacies and a suggested service for patients with migraine.

Results: Most of the pharmacists were satisfied with the services they offer. The patients are also satisfied, they said. The informants were not just offering the services for the income of the pharmacy; they really wanted to help the patients. They are especially gratified with the guidance of inhalation of patients with asthma or Chronic obstructive pulmonary disease. When it comes to vaccination, the informants are divided in two parts: the pharmacists who have offered this service for a while were much more positive than the pharmacists who were not offering this service yet. Although migraine patients is an important patient group in the pharmacy, only half part of the informants gave information and other advice, and asked if the migraine patient was satisfied with the medication, how did they use the medicine and so on.

Less than $50 \%$ of the informants demonstrated use of the different formulations, due to lack of knowledge. The recruited pharmacists were positive to a professional service for the migraine patients.

Conclusion: The pharmacists that participated in this study were positively oriented to professional services in the pharmacy. Not only for helping the patients to use their medicines more correctly, but also for using more of their knowledge. They were warmly welcoming of a similar service for migraine patients.

Disclosure of Interest: None Declared.

\section{CP-PC035: An evaluation of mental health clinical pharmacist prescribers within primary care medical practices in NHS Highland}

K. Maclure ${ }^{1, *}$, D. Stewart ${ }^{1}$, E. Buist ${ }^{2}$, R. McLelland ${ }^{2}$, A. MacLure ${ }^{1}$, S. Cunningham ${ }^{1}$, K. Macaskill ${ }^{2}$, K. Gibson Smith ${ }^{1}$, G. Rushworth ${ }^{2}$

${ }^{1}$ School of Pharmacy and Life Sciences, Robert Gordon University, Aberdeen, ${ }^{2} \mathrm{NHS}$ Highland, Inverness, United Kingdom

Background and objective: A pilot has been conducted during which a specialist mental health clinical pharmacist prescriber (SMHCPP) consulted with patients by appointment at one of two GP Practices. The aim of this study was to evaluate the SMHCPP delivered pharmaceutical care to patients with anxiety disorders and/ or depression in NHS Highland.

Setting and method: Both pharmacists handed out a study pack to patients. Patient study packs included a questionnaire based on the CARE Measure plus 5-point Likert scale attitudinal statements all subject to statistical analysis. Ethical approval had been gained.

Main outcome measures: Patient satisfaction measured with the validated CARE scale.
Results: Eleven of 70 surveys were returned (response rate 16\%) with an even gender split. Most were employed $(n=9)$. Wellbeing was good $(n=3)$, fair $(n=6)$ or poor $(n=2)$. All $(n=11)$ thought the pharmacist excellent at 'making you feel at ease', 'really listening', 'being interested in you as a whole person', 'showing care and compassion', 'being positive', 'explaining things clearly'. Most said the pharmacist was excellent at 'letting you tell your story' $(n=10)$, 'fully understanding your concerns' $(\mathrm{n}=10)$, 'helping you take control' ( $n=9)$ and 'making a plan of action with you' $(n=8)$. All $(\mathrm{n}=11)$ strongly agreed/agreed they were 'confident that a pharmacist will prescribe as safely as a GP' also that they wanted their 'pharmacist and doctor to work together to make sure I am receiving the best treatment'. However, 'given the choice, I prefer to consult a GP rather than a pharmacist' half $(n=5)$ were unsure, some strongly agreed $(n=1) /$ agreed $(n=1)$ but others strongly disagreed $(n=2)$ / disagreed $(\mathrm{n}=2)$.

Conclusion: Overall, this small sample from a difficult to reach population were positive about care they received from the SMHCPP. Disclosure of Interest: None Declared.

CP-PC036: Development of a tool to support community pharmacy engagement with patients who may be homeless

\section{K. Maclure ${ }^{1, *}$, K. Gibson Smith ${ }^{1}$, V. Paudyal ${ }^{2}$, D. Stewart ${ }^{1}$}

${ }^{1}$ School of Pharmacy and Life Sciences, Robert Gordon University, Aberdeen, ${ }^{2}$ Institute of Clinical Sciences, School of Pharmacy, University of Birmingham, Birmingham, United Kingdom

Background and objective: Homelessness remains a global public health concern. A prior survey indicated the need for pharmacist training and coverage of the topic of homelessness around minimising impact of medicines use, referring for social support, confidence in broaching the subject with patients, support and guidelines for practice.

Purpose

To further explore community pharmacists' views on the need for, type and content of a tool to support their engagement with patients who may be homeless.

Setting and method: A semi-structured interview schedule was developed based on existing literature, survey results and theoretical frameworks (Theoretical Domains Framework; COM-B model) Community pharmacists from England and Scotland who had taken part in a survey consented to take part in a follow-on, digitally recorded telephone interview. Each was transcribed then coded using a framework approach. Ethical approval had been gained.

Main outcome measures: Community pharmacist views on the need for and content of a tool to support engagement with patients who may be homeless.

Results: Interviews $(n=15)$ conducted November-December 2017 found all participants felt capable and were motivated to improve their approach when engaging with patients who may be experiencing homelessness. They welcomed the opportunity to contribute to the content and format of a support tool. They also thought the topic should be better covered at undergraduate and continuing professional development. Some suggested role play to improve confidence and all felt capable if provided with up-to-date information on local homelessness support services.

Conclusion: Community pharmacists are capable, motivated and have the opportunity to better engage with patients considered to be homeless. However, this was a small cohort and further research is indicated to inform support tool design.

Disclosure of Interest: None Declared. 
CP-PC037: Pharmaceutical care in pediatric patients, knowledge attitudes and practices of community pharmacists in Jordan

\section{T. L. Mukattash ${ }^{1, *}$, M. Qudah ${ }^{1}$, I. Alshammari ${ }^{1}$ \\ ${ }^{1}$ Department of Clinical Pharmacy, Jordan University of Science and Technology, Irbid, Jordan}

Background and objective: Advances in paediatric medicine, increasing complexities of childhood disease, and unique dosing and pharmacokinetic challenges all support a growing need for skilled and experienced pharmacists in pediatric pharmacy. The aim of this study was to determine pharmacists' perceived knowledge and expertise required to make recommendations regarding selected pediatric topics.

Setting and method: The questionnaire was developed after an extensive literature review of studies that assessed healthcare professionals' knowledge of pediatrics. The questionnaire was assessed for face and content validity by being sent to a panel of experts and was the piloted among 20 pharmacists.

After obtaining the required administrative and ethical approvals, the questionnaire was distributed to 1460 pharmacists practicing in community and hospital settings. The questionnaire explored their perceived knowledge, expertise, and comfort in providing recommendations related to different pediatric topics.

The questionnaire consisted of four sections: (1) Demographics (2) Perceived knowledge of pediatric treatment and dosing (3) Real life pediatric cases (4) Future aspirations to enhance pediatric pharmacy. Main outcome measures: A total knowledge score was calculated by summing the correct answers for each case, creating a scale of zero to ten, where 10 is the highest knowledge score and zero was the lowest knowledge score.

Results: Of 1460 questionnaires, 1000 questionnaires where complete and returned (68.5\%). The majority of respondents (76\%) practiced in a community setting. The majority of respondents reported that more than $30 \%$ of their patients were pediatrics. In general, respondents had a good perception of their knowledge and expertise to make recommendations for the frequently occurring conditions among pediatric patients. Work experience and peer influence had the most influence on their knowledge in pediatrics.

The majority of respondents scored an average knowledge score in pediatric knowledge (64.6\%), while $26.3 \%$ had a low knowledge score, and only $9.1 \%$ of respondents had a good knowledge score in pediatrics. This was highly influenced by holding higher education degrees, specifically in clinical pharmacy, and being working in a hospital setting. Conclusion: The present study sheds the light on an alarming lack of knowledge in pediatric treatments amongst community and hospital based pharmacies in Jordan. Though pharmacists reported high confidence in their ability to work with this age group, knowledge scores highlighted how deficient their pediatric knowledge is.

Pediatric pharmacy certification, specialized postgraduate education, specialized experiential training and continuous professional development top the list for solutions suggested to address this defect. Furthermore, developing the undergraduate pharmacy curriculum to cover specialized topics, such as pediatrics seems to be very important.

Disclosure of Interest: None Declared.

\section{CP-PC038: Identification of medication-related problems} among the elderly nursing homes residents

\section{A. Olearova ${ }^{1,2, *}$, L. Duban ${ }^{1}$}

${ }^{1}$ Department of Organization and Management in Pharmacy, Faculty Of Pharmacy, Comenius University In Bratislava, ${ }^{2}$ Department of Clinical Pharmacology, University Teaching Hospital Bratislava Ruzinov, Bratislava, Slovakia
Background and objective: Polymorbidity and polypharmacy are very common for older age and may give rise to medication-related problems. The aim of our study was to identify and analyse medication-related problems among the elderly-residents of nursing homes by a clinical pharmacist.

Setting and method: In our study, we have analysed the medication prescriptions and records in three nursing homes residents in Slovakia. We have used STOPP/START criteria, summaries of product characteristics, and Lexicomp ${ }^{\circledR}$ database as well to identify medication-related problems. Every identified medication-related problem was recorded and discussed the more appropriate solutions with the prescribers-general practitioners. One month after the pharmacist's intervention we have visited the nursing homes again to investigate the level of acceptance of the pharmacist's recommendations.

Main outcome measures: We have focused and noticed the modeication-related problems, such potentially inappropriate medication use in older adults, inappropriate dosing, medication interactions, length of therapy, properly using of drugs, and adverse drug reactions. Results: We have analysed medication prescriptions and medication use in 57 seniors, residents of nursing homes ( 39 females, 18 males), in range 69-93 years, average age $78.7 \pm 5.6$. We have identified 307 medication-related problems (5.4 per patient in average):

1. inappropriate prescribed medication $(n=67)$-mainly antidepressants, antipsychotics, vasodilators, diuretics, antispasmodics, and laxatives;

2. inappropriate dosing $(\mathrm{n}=37)$-mainly benzodiazepines and analgesics;

3. potentially drug interactions $(\mathrm{n}=103)$-mainly with proton pump inhibitors, warfarin, theophylline, analgesics, and calcium channel blockers;

4. manifested adverse drug reactions $(n=31)$-mainly in diuretics, laxatives and antispasmodics;

5. inappropriate using/application of medication $(n=27)$ - mainly in modified-released dosage forms;

6. inappropriate length of pharmacotherapy $(n=31)$-mainly in benzodiazepines, contact laxatives and non-steroidal antiinflammatory agents;

7. others $(\mathrm{n}=11)$.

One month after the pharmacist's intervention we have found out, that from 124 recommendations were accepted 55 (44.4\%). The dosing was corrected in 23 cases, inappropriate medication was stopped in 11 cases and inappropriate using/application was corrected in 21 cases. Conclusion: Our results suggest, the pharmacist could contribute very greatly to medication-related problems identification and prevention. The multidisciplinary team-nurses, physicians, pharmacists and other care givers could support the efficacy and safety of pharmacotherapy in elderly in nursing homes.

Disclosure of Interest: None Declared.

CP-PC039: Assessment of individuals' knowledge and attitudes towards utilization of analgesics at community pharmacy setting

B. Torun ${ }^{1}$, O. Ozkan ${ }^{1, *}$, H. Zortul ${ }^{1}$, S. Kesedar ${ }^{1}$, F. V. Izzettin ${ }^{1}$, M. Sancar ${ }^{1}$, B. Okuyan ${ }^{1}$

${ }^{1}$ Department of Clinical Pharmacy, Marmara University Faculty of Pharmacy, Istanbul, Turkey

Background and objective: The aim of the study was to evaluate the knowledge and attitude about the use of analgesics and elucidate the relation with participants' sociodemographic characteristics and beliefs about medications at community pharmacy setting.

Setting and method: This study was conducted with adult patients who came to pharmacy with an analgesic request between December 
2017 and January 2018in a community pharmacy located in Ankara, Turkey.

Main outcome measures: Sociodemographic characteristics and medical history of patients were collected. Beliefs about analgesics of patients were investigated via Beliefs about Medicines QuestionnaireTurkish version (BMQ-T, scored between 18-80) which is consisted of four sections: General Harm (GH, scored between 4-20), General Overuse (GO, scored between 4-20), Specific Necessity (SN, scored between 5-25), Specific Concerns (SC, scored between 5-25) ${ }^{1,2}$. The level of analgesic knowledge was evaluated through a questionnaire (scored between 0-8) developed by McPherson ${ }^{3}$.

Results: Among total of 140 individuals ( $31.4 \%$ male), the mean of age was calculated as $39.02 \pm 12.62$. The mean duration of participants' education was $11.66 \pm 4.85$ years. The mean total score of Beliefs About Medicines Questionnaire-Turkish version was calculated as $52.10 \pm 12.00$ (male $50.66 \pm 13.07$; female $52.77 \pm 11.50$ ) and the mean of medication knowledge level score was calculated as $6.03 \pm 1.65$ (male $5.65 \pm 1.94$; female $6.20 \pm 1.49$ ). The most preferred analgesic among the participants was found as paracetamol $(41.4 \%)$. The results indicates that when the years in education increased, medication knowledge level score also increased $(p<0.01)$. On the other hand, a decrease was observed in BMQ-SN, BMQ-SC and total BMQ score with an increase in years in education $(p<0.01)$.

Conclusion: It was determined that the participants with longer time spent in education had moderate-high knowledge level with less beliefs on analgesics. It is encouraging that the most preferred analgesic was paracetamol, which is safer than other analgesicsconsidering the individual condition of the patient-in terms of the risk of interaction and side effects. However, the pharmacist should instruct the individuals on the prevention of inappropriate analgesic use to avoid unnecessary drug use and to maintain rational drug use.

\section{References}

1. Horne R, Weinman J, Hankins M. The Beliefs about Medicines Questionnaire: the development and evaluation of a new method for assessing the cognitive representation of medication. Psychol Health 1999;14:1-24.

2. Cinar M, Cinar FI, Acikel C, Yilmaz S, Çakar M, Horne R, Simsek I. Reliability and validity of the Turkish translation of the beliefs about medicines questionnaire (BMQ-T) in patients with Behçet's disease. Clin Exp Rheumatol. 2016 Sep-Oct;34 Suppl 102(6):46-51.

3. McPherson, M. L., et al. (2008). "Association between diabetes patients' knowledge about medications and their blood glucose control." Res Social Adm Pharm 4(1):37-45.

Disclosure of Interest: None Declared.

\section{CP-PC040: Evaluation of patients' knowledge and attitudes towards utilization of oral anticoagulants at community pharmacy setting}

\section{O. Ozkan $^{1, *}$, A. Basakil ${ }^{1}$, B. Torun ${ }^{1}$, M. Sancar ${ }^{1}$, B. Okuyan ${ }^{1}$}

${ }^{1}$ Department of Clinical Pharmacy, Marmara University Faculty of Pharmacy, Istanbul, Turkey

Background and objective: In this study, it is aim to assess the knowledge and attitudes of patients towards oral anticoagulant medications.

Setting and method: Our descriptive study conducted with 108 patients aged 18 and over who have taking any oral anticoagulant medication for at least 4 weeks at community pharmacy setting between November 20, 2017 and March 20, 2018.
Main outcome measures: The Oral Anticoagulation Knowledge (OAK) test Turkish validated version of OAK, consists of 20 items and 5 dimensions including monitoring, drug-drug interactions, basic drug information, adverse effects and nutrition problems, was used in this study. Patients' adherence and beliefs was evaluated according to 'Morisky Green Levine Medication Adherence Scale' and Beliefs about Medicines Questionnaire-Turkish version (BMQ-T), consists of four sections: General Harm (GH, 4 item), General Overuse (GO, 4 item), Specific Necessity (SN, 5 item), Specific Concerns (SC, 5 item) respectively.

Results: More than half of the patients were female $(54.6 \%)$ and the mean age of the patients was $59.92 \pm 11.92$ (37-86). The only $28.7 \%$ of them had university graduation. The average score for The OAK test was $6.86 \pm 1.85(3-12)$. The highest $(74.1 \%)$ correct response rate of the question was 'It is important for a patient on Coumadin (warfarin) to monitor for signs of bleeding' and the lowest (2.8\%) was 'When it comes to diet, people taking Coumadin (warfarin) should'. The total mean score for the Morisky Green Levine Medication Adherence Scale was $2.38 \pm 1.01$. The mean score of BMQ-T for each sections were $3.74 \pm 0.48, \quad 3.96 \pm 0.39, \quad 3.31 \pm 0.52$, $2.97 \pm 0.56$ for SC, SN, GO and GH, respectively.

Conclusion: Most patients who are under warfarin treatment are aware of the bleeding risk and the importance of monitoring its signs, however their knowledge about the appropriate diet with warfarin is inadequate. To improve the knowledge of patients regarding oral anticoagulation, pharmacists should provide a detailed patient education about warfarin including dietary recommendations at community pharmacy setting.

Disclosure of Interest: None Declared.

CP-PC042: Impact of pharmacists-led medication reviews: an action plan for Spanish community pharmacies

\section{Revilla ${ }^{1, *}$, F. Miranda ${ }^{1}$, P. Modamio ${ }^{2}$}

${ }^{1}$ Pharmacy Care Services Department, Official Pharmaceutical Society of Santa Cruz de Tenerife, Santa Cruz de Tenerife, ${ }^{2}$ Clinical Pharmacy and Pharmacotherapy Unit, Faculty of Pharmacy and Food Sciences, University of Barcelona, Barcelona, Spain

Background and objective: Pharmacists are becoming ever more closely involved in patient care. An important tool for them is the Medication Use Review, enabling interventions that improve care and optimize clinical outcomes. The purpose of this study was to look at medication reviews from around the world in order to assess the feasibility of implementing a pharmacist-led intervention service in Spanish community pharmacies. It also looked at what would be required for a randomized trial to assess short and long-term outcomes.

Design: A literature search was performed on PubMed, from January 1, 2011 up to February 2, 2018. This looked for pharmacist-led intervention studies carried out in care settings all around the world. Only interventions involving both a medication review and face-toface communication were included. Summaries of the studies were included, with details of their title, objective, design, setting, target population, description, outcomes, cost and benefit assessments, challenges and opportunities, country and references.

Results: 106 relevant articles were found, referencing 21 separate studies. The majority of these studies (14 of 21) showed an observational analysis design. Most (12 of 21) were carried out in primary care centres. Twelve showed positive benefits on patient outcomes by decreasing drug-related problems such as dosing, side effects, drug indication or overuse. Seven studies were conducted reviewing polymedicated patients, and three of them were followed by interventions for patients with diabetes. Four studies showed improvements in clinical outcomes, related to blood pressure or A1c 
hemoglobin levels. However, only two studies reported hard outcomes such as hospital admissions. In addition, patients became more involved in the intervention and showed improvements in satisfaction. Conclusion: This study looked at a variety of interventions in which pharmacists and other health professionals worked together to improve patient health. When these interventions were well stated, there were generally positive impacts on drug-related problems, adherence, quality of life and clinical values. A randomized controlled trial would provide better data on all these aspects, and let us know whether it would be cost-effective in a Spanish context. Such a trial would require a unified conceptual and methodological framework, as well as training of Spanish community pharmacists.

Disclosure of Interest: None Declared.

\section{CP-PC043: Determination of knowledge and attitudes} of community pharmacists towards probiotics

\author{
N. Poyraz ${ }^{1}$, B. Okuyan ${ }^{1}$, K. N. Cengiz ${ }^{1}$, F. V. Izzettin ${ }^{1}$, \\ M. Sancar ${ }^{1, *}$ \\ ${ }^{1}$ Clinical Pharmacy, Marmara University Faculty of Pharmacy, \\ Istanbul, Turkey
}

Background and objective: Aim of this study is to evaluate the knowledge and attitudes of the community pharmacists towards probiotics.

Setting and method: The study was conducted between 15 November 2017 and 15 March 2017. According to sample size calculation 80 of the community pharmacists registered at Ordu Chamber of Pharmacy, Turkey, was included in this study. The study was performed by face to face interview.

Main outcome measures: Self-structured validated questionnaire with 33 items (Cronbah's alpha is 0.746) was applied for data collection.

Results: The mean age of participants was $41.78 \pm 14.38$. The mean of pharmacists' professional years was $18.81 \pm 13.74$. Of them, $\% 56.25$ was female. Of all pharmacists, $91.2 \%$ and all male pharmacists $(100 \%)$ participating in the survey agreed that probiotics had benefit effect. However, $87.3 \%$ of pharmacists thought that evidencebased information regarding probiotics were limited. Of them, 73.4\% claimed that pharmaceutical care services should be provided to patients regarding probiotics. On the other hand, the pharmacist, who had professional years less than 10 , more likely declared the necessary of providing pharmaceutical care regarding probiotics when compared with pharmacists who had professional years 10 or more $(p<0.05)$. Of them, $51.2 \%$ believed that they correctly and effectively instructed their patients about probiotics. Female pharmacists more likely advised their patients to concomitantly use of probiotics with antibiotics when compared with male pharmacists $(p<0.05)$. Of them, $98.8 \%$ reported that they did not receive any negative feedback from the patients who they offered probiotic use.

Conclusion: According to the results of this study, community pharmacists mostly thought probiotics as beneficial and effective. Most of the pharmacists noticed the importance of pharmaceutical care services for probiotics.

Disclosure of Interest: None Declared.

\section{CP-PC044: Evaluation of knowledge and attitudes of patients} and pharmacists on using herbal products

\section{S. Tezcan ${ }^{1}$, M. Kızılkaya ${ }^{1}$, M. Sancar ${ }^{1, *}$, F. V. İzzettin ${ }^{1}$ \\ ${ }^{1}$ clinical pharmacy, Marmara University, istanbul, Turkey}

Background and objective: Herbal products are a part of treatments referred as complementary and alternative medicine (CAM) and have been used over 1000 years (1). The aim of our study is to evaluate the knowledge and attitudes of patients and pharmacists related to the use of herbal products.

Setting and method: 201 patients who applied to community pharmacies and 52 pharmacists in Turkey, between December 2017 and February 2018, were included. Two self structured questionnaires were prepared, one of them consisted 12 items for patients and the other one consisted 15 items for the pharmacists. Face-to-face questionnaires were applied to patients and community pharmacists to assess the knowledge level and attitudes related to the use of herbal products.

Main outcome measures: Evaluation of the patients' and pharmacists knowledge and attitudes about herbal products.

Results: The mean age of the patients was $34.6 \pm 1.1(18-83)$ and $68.2 \%(n=37)$ of them were female. Sixty-nine point two percent $(n=139)$ of the patients were using herbal products and $46.8 \%$ of the them believed that the use of herbal products were completely harmless $(p=0.001)$. Sixty-five point seven percent $(\mathrm{n}=132)$ of the patients had chronic diseases and $76.52 \%(n=101)$ of these patients were using herbal products $(p=0.02)$. It was found that $52.9 \%$ $(n=27)$ of pharmacist were female and $27.5 \%(n=14)$ of them have been in service for 20-25 years.

In our study $90.2 \%(\mathrm{n}=46)$ of pharmacists was selling herbal products. It was determined that patients were asked pharmacists at least once a week about the use of herbal products $(45.1 \%, \mathrm{n}=23)$ $(p<0.001)$.

Conclusion: In our study, it was observed that the use of herbal products was more common in patients with chronic illnesses, and the majority of patients believed that these products were harmless. We believe that pharmacists will significantly contribute to the rational and safe use of herbal products as an health advisor.

Disclosure of Interest: None Declared.

CP-PC045: TRIAGE: a practical tool for identifying and discussing medication problems in the pharmacy

L. Van Dijk ${ }^{1}$,*, M. Vervloet ${ }^{1}$, H. Zwikker ${ }^{2}$, A. J. Linn ${ }^{3}$, A. Lamboo ${ }^{4}$, E. Koster ${ }^{5}$, S. Gipmans ${ }^{6}$

${ }^{1}$ NIVEL, ${ }^{2}$ IVM, Utrecht, ${ }^{3}$ ASCoR, Amsterdam, ${ }^{4}$ Center for Patient and Medication, Loenen, ${ }^{5}$ Utrecht University, ${ }^{6}$ Benu Pharmacies, Utrecht, Netherlands

Background and objective: Background and objective: The pharmacy counter is a good place to identify, discuss and possibly solve medication-related problems. Previous research shows that communication during pharmacy encounters can be improved. Practical tools that support pharmacy technicians to initiate a conversation with the patient are missing. The aim of this study is to develop and test a practical set of questions that support pharmacy technicians to identify problems during the encounter, including an overview of potential problems with possible solutions (TRIAGE).

Setting and method: TRIAGE was developed based on literature, focus groups with pharmacy technicians and pharmacists and input from experts. In 10 BENU community pharmacies, 17 pharmacy technicians conducted the TRIAGE interview with patients who collected cardiovascular medication for themselves. This involved an encounter for a first repeat prescription or a follow-up encounter for which it was known that the patient collected the medication too early or too late. For each encounter, identified problems, suggested solutions and whether the pharmacy technicians themselves could act on/ deal with the problem?

Main outcome measures: $\%$ of encounters where a medication-related problem was identified.

Results: A total of 105 TRIAGE interviews were held, 66 for first repeat prescription and 39 for a follow-up encounter. In 15 (23\%) of 
the encounters for a first repeat prescription, a problem was identified. These problems concerned patients who forgot to take their medication, who found the medication regime complex or who had (fear of) side effects. In 10 encounters the problem was dealt with on the spot. In three (8\%) follow up encounters, a problem was identified. Twice, this problem referred to the lack of clarity on the duration of the medication and one time it referred to forgetting medication. Two problems were solved on the spot. Technicians found TRIAGE a useful tool for asking open questions, but indicated that patients are not always open to conversation.

Conclusion: TRIAGE provides the pharmacy technician with a practical tool to start the conversation about medication use. A substantial number of problems were identified with TRIAGE during first repeat prescriptions.

Disclosure of Interest: None Declared.

CP-PC046: Medication use evaluation by community pharmacists: impact on patient-reported outcomes

\section{J. Wuyts ${ }^{1, *}$, J. Maesschalck ${ }^{2}$, I. De Wulf ${ }^{2}$, J. De Lepeleire ${ }^{3}$, V. Foulon ${ }^{1}$}

${ }^{1}$ Clinical Pharmacology and Pharmacotherapy, KU Leuven, Leuven, ${ }^{2}$ Association of Belgian Phamacists, Brussels, ${ }^{3}$ Academic Centre of General Medicine, KU Leuven, Leuven, Belgium

Background and objective: This study evaluated the impact of a medicines' use review for elderly patients.

Setting and method: Between December 2016 and May 2017, 57 Belgian community pharmacies participated in a pilot project and performed a medicines' use review (intermediate medication review) for aged polymedicated patients. A before-after design was used to measure the impact of the pharmacists' intervention on patient-reported outcomes before, 3 and 12 weeks after the intervention. The questionnaires focused both on humanistic (medicines related quality of life, patient satisfaction, self-management) and clinical outcomes (adherence).

Main outcome measures: The medicines related quality of life was considered as the primary outcome and was measured using the Living with Medicines Questionnaire (LMQ) version 3.

Results: Questionnaires at baseline and endpoint were available for 83 patients of 24 pharmacies. The majority of the respondents were female (50/83) and the median age was 77 years. The median number of drugs was 7 and half of the respondents used medication three or more times per day (43/82). Only a minority of patients received help with their medication $(19 / 83)$.

The median LMQ scores evolved from $83.5 / 200$ at baseline to $84.0 / 200$ 3 weeks later. This small increase in LMQ score could indicate higher medication burden, potentially due to increased awareness or changes in medication regimen. Scores significantly reduced 3 months after the review to $79.5 / 200$. The median medication burden score on the visual analogue scale was low (1/10) and remained unchanged throughout the process.

The Probabilistic Medication Adherence Scale (PROMAS) $(n=67)$ showed high adherence scores at baseline (14/18) which remained unaltered 12 weeks later. Overall, the number of patients with increased adherence was higher than the number of patients in which adherence was reduced $(n=29$ versus $n=23$; difference not statistically significant). The Patient Satisfaction with Pharmacist Services Questionnaire (PSPSQ 2.0), demonstrated that patient's health concerns were fully addressed, useful recommendations for medication use were provided and the information was considered understandable and reliable by over $70 \%$ of the respondents.

Conclusion: The medication review service resulted in high patient satisfaction and a trend towards reduction in medication burden. However, adherence scores were high and medication burden was relatively low at baseline.

Disclosure of Interest: None Declared.
CP-CE003: Antibiotic consumption evaluation in a general hospital center

\section{F. Badibouidi ${ }^{1, *}$, L. Lesec ${ }^{1}$, N. Landgraf ${ }^{2}$, P. Duchesne ${ }^{1}$ \\ ${ }^{1}$ Pharmacy, ${ }^{2}$ Infectious diseases, GHPSO, Creil, France}

Background and objective: As part of the evaluation of professional practices, we conducted to investigate the systemic antibiotic consumption. The objective was to assess current antibiotic patterns use in our hospital.

Design: Antibiotic consumption data during 2016 and 2017 were extracted from the economic and financial software "magh2" and used. Antibiotic prescription data was classified using the ATC system and converted into DDD. Consumption figures were presented as the number of DDD per 1000 inhabitants per day (DID).

A prevalence survey of antibiotic use was also carried out with data abstracted principally from patient medical records supplemented by interviews with physicians when needed. The pattern of antibiotic use was analyzed. Detailed information on antibiotic consumption was also explored (indication, dosage, duration, administration route, prescription reassessment at 48-72 $\mathrm{h}$, documented treatment).

Results: The total consumption increased from 526 to 550 DID in 2017 compared to 2016. The most frequently prescribed antibiotic was amoxicillin/clavulanic acid (191 and 170.7 DID, in 2017 and 2016, respectively), followed by amoxicillin (110 and 78.8 DID), ceftriaxone (60.8 and 52.5 DID) and metronidazole (45 and 40.4 DID). The most prescribed classes of antibiotics were betalactamins (440.39 and 404.48 DID), fluoroquinolones (77 and 70 DID), imidazoles (45 and 40 DID), macrolides (31 and 19 DID).

The prevalence survey was conducted on a given day in the departments of digestive surgery, geriatrics, general medicine, oncology, pediatrics and resuscitation.

Among the patients surveyed (158 patients), 34.8\% (55 patients) had antibiotics.

The most prescribed antibiotics were amoxicillin/clavulanic acid $(28.16 \% ; 20 / 71)$, ceftriaxone $(19.7 \% ; 14 / 71)$ and metronidazole $(11.26 \% ; 8 / 71)$. The dosage of antibiotics was generally seen as optimal. $36.4 \%$ of prescriptions were documented, $48 \%$ were reassessed at $48-72 \mathrm{~h}$ and $35.6 \%$ were per os administration. The treatment duration was specified in $40 \%$ of cases.

Conclusion: The consumption of antibiotics is very high in our hospital-it is comparable to that observed in a university hospital center.

This work helped to make an inventory of antibiotic use and highlighted areas for improvement: the prescription duration and its reassessment, the change to per os as soon as possible and to seek the opinion of the specialist in infectious diseasemore frequently.

Disclosure of Interest: None Declared.

CP-CE005: Preventing potentially inappropriate prescribing in the older person-the views of community pharmacists C. R. Hansen ${ }^{1,2}$, S. Byrne ${ }^{1}$, D. O'Mahony ${ }^{3,4}$, P. M. Kearney ${ }^{5}$,
L. J. Sahm ${ }^{1,6, *}$

${ }^{1}$ Pharmaceutical Care Research Group, School of Pharmacy, University College Cork, Cork, Ireland, ${ }^{2}$ Section for Social and Clinical Pharmacy, Department of Pharmacy, Faculty of Health and Medical Sciences, University of Copenhagen, Copenhagen, Denmark, ${ }^{3}$ Department of Medicine, University College Cork, ${ }^{4}$ Department of Geriatric Medicine, Cork University Hospital, ${ }^{5}$ School of Public Health, University College Cork, ${ }^{6}$ Pharmacy Department, Mercy University Hospital, Cork, Ireland

Background and objective: Older people ( $\geq 65$ years) are at increased risk of receiving potentially inappropriate prescribing (PIP), 
resulting in higher risk to patient safety. No intervention to date has successfully reduced the substantial PIP prevalence in primary care despite the availability of explicit criteria sets to identify PIP, the two most cited sets being; Beers criteria and STOPP/START criteria. Pharmacist-led PIP prevention interventions in hospitals have shown positive results and interventions in primary care settings might similarly benefit from pharmacist involvement. This study aimed to explore community pharmacists' views on their role in preventing PIP and the perceived barriers and facilitators to implementing PIP prevention in community pharmacy practice.

Setting and method: Semi-structured interviews with community pharmacists in southern Ireland. The theoretical domains framework (TDF) was used to design the topic guide and to analyse the interview transcripts. Matrix sampling was used to recruit a participant sample representative of pharmacists working in Ireland. Interviews were conducted until the point of thematic saturation was reached. Domains of highest relevance for PIP prevention were determined and reported. Ethical approval was obtained for the study.

Main outcome measures: Barriers and facilitators to implementing PIP prevention in community pharmacy practice.

Results: 18 interviews were conducted with pharmacists whose median age was 30 years (IQR 27-35) and whose median level of postgraduate experience was 6 years (IQR 3-8). Seven of the 14 domains were found to be relevant to PIP prevention. Pharmacists expressed uncertainties about the extent of their role in preventing PIP but welcomed more involvement. Perceived barriers to PIP prevention in community pharmacists were deficient information sharing, collaboration and communication between pharmacists and medical doctors. Up-to-date guidelines and education were perceived facilitators of PIP prevention, but pharmacists expressed the need for treatment guidelines to be applied by everyone and guidance on how to manage PIP rather than PIP identification alone.

Conclusion: Pharmacists welcomed a potential role in PIP prevention but expressed a need for more guidance and education on how to manage identified PIP. Pharmacists suggested continuous and up-todate training in guidelines and PIP prevention as well as improved collaboration with doctors.

Disclosure of Interest: None Declared.

CP-CE006: Guidance of patients with newly diagnosed type II diabetes on monitoring of blood glucose in Norwegian pharmacies

\section{I.-L. Neslein ${ }^{1, *}$, T. Krogstad ${ }^{1}$}

${ }^{1}$ Natural Sciences and Health, Oslo Metropolitan University, Oslo, Norway

Background and objective: Self-monitoring of blood glucose (SMBG) is considered an integral part of glycemic control and diabetes management. In order to obtain satisfactory quality of SMBGlevels, standardized user training, understanding of the analytical procedure and knowledge of interpretation of the test results are required. Pharmacists have a responsibility for the training and counseling of these patients to avoid suboptimal glycaemic control and increased risk of complications as consequences. This study aims to map training procedures and routines regarding SMBG offered to newly diagnosed patients Type2-diabetes by pharmacies in Norway to point to an important area for improved pharmacy care.

Setting and method: In this pilot study, pharmacists in 26 pharmacies were interviewed regarding guidance of patients with newly diagnosed Type2-diabetes and glycaemic control management in two Norwegian counties between June 2017 and April 2018. There were 1-3 pharmacists employed at each pharmacy, handling between 2200 and 26,000 prescriptions per year.

Main outcome measures: The mixed-method approach of quantitative descriptive data and qualitative concerns from the pharmacists.
Results: All 26 pharmacies offer at least three different BG-instruments and deliver them free of charge to patients with prescriptions for sampling equipment. The patients are free to choose instrument, regardless of selling price. All the pharmacies are reimbursed by the suppliers. All 26 pharmacies teach newly diagnosed diabetes patients in BG-level testing, and were educated by supplier representatives and/or colleagues. One pharmacist had attended supplier-neutral courses related to SMBG. Three pharmacists had some knowledge of QCs related to the instruments, but none had used it or discussed it with patients. None of the pharmacists could recall explicit training in quality assessment related to capillary blood sampling and related QC procedures as pharmacy students.

Conclusion: Lack of standardized educator and user SMBG-training, as well as QC-implementation, may lead to erroneous test results and incorrect interpretation. This may in turn lead to inadequate treatment and increased risk of disease related complications. Increased focus on quality control will contribute to improved pharmacy care and lead to more effective and safer monitoring of patients by improving SMBG in patients.

Disclosure of Interest: None Declared.

CP-CE007: Pharmacy students' experiences of a four-week mindfulness course: qualitative results of a mixed methods study M. O'Driscoll ${ }^{1}$, S. Byrne ${ }^{1}$, S. Lambert ${ }^{2}$, L. J. Sahm ${ }^{1,3, *}$ ${ }^{1}$ Pharmaceutical Care Research Group, ${ }^{2}$ School of Applied Psychology, Univeristy College Cork, ${ }^{3}$ Pharmacy Department, Mercy University Hospital, Cork, Ireland

Background and objective: Pharmacy students demonstrate higher stress levels than the general population, regardless of year of study. ${ }^{1}$ This can be linked to subsequent stress as a healthcare practitioner, leading to depression and burnout, and impacting upon patient care. Mindfulness, defined as "paying attention in a particular way, on purpose, non-judgmentally, to the present moment" 2 is a braintraining technique that changes how one relates to stress, and may be a suitable way to teach pharmacy students stress management and self-care ${ }^{3}$. The aim of this study was to assess the effect of a 4-week mindfulness course on pharmacy student stress and wellbeing, and determine students' views of the course content and design.

Setting and method: Undergraduate pharmacy students in University College Cork who had completed at least $75 \%$ of the mindfulness course offered during the 2016/2017 academic year were invited to participate in follow-up interviews. Local ethics approval was obtained. Recruitment occurred via email. Interviews were audio recorded, anonymized and transcribed by the researcher (MOD).

Main outcome measures: Transcripts were analysed using the Braun and Clarke method of thematic analysis ${ }^{4}$, to allow for the natural emergence of themes. Data were coded using QSR International NVivo Qualitative Data Analysis Software Version 11.

Results: A total of 21 course participants (62\% female) representing all year groups were interviewed in March 2017. The following five themes emerged: (a) increased daily awareness, (b) positive changes in the effect of stress, (c) the course is not for everybody, (d) benefits of the group dynamic, (e) prevention is better than cure.

Conclusion: Interview participants reported improvements in their wellbeing and ability to manage stress as a result of taking part in the mindfulness course. The study has also provided useful insights from the student perspective about how best to bring this research forward such as introduction to the concept in earlier years to prevent the onset of academic stress, and an opportunity to try it before signing up. Disclosure of Interest: None Declared. 
CP-CE008: Trainees, tutors and employers' views and experiences of the NHS Education for Scotland, Preregistration Pharmacist Scheme (PRPS) modular programme

\section{F. E. Woodley ${ }^{1, *}$, R. Edwards ${ }^{2}$, G. Craig ${ }^{1}$, A. Power ${ }^{1}$}

${ }^{1}$ NES Pharmacy, NHS Education For Scotland, Glasgow, ${ }^{2}$ School of Pharmacy and Life Sciences, Robert Gordon University, Aberdeen, United Kingdom

Background and objective: The Pharmacy pre-registration year is a final training year in practice following 4 years at university. The Preregistration Pharmacist Scheme (PRPS), delivered by NES provides trainees with a standardised programme during which they evidence their progression to consistently meet the performance standards set by the General Pharmaceutical Council. This programme traditionally delivers training in one pharmacy setting.

Responding to the evolving role of pharmacy and the need for pharmacists to work seamlessly across pharmacy settings, as emphasised in 'Achieving Excellence in Pharmaceutical Care', NES developed a modular programme to allow training across sectors to begin at an early stage in the career of a pharmacist.

In 2013-14, NES initiated a modular pre-registration programme consisting of three separate blocks of training, within different pharmacy settings: including 6 months in community pharmacy and 4 months in hospital pharmacy. This project explored views, experiences and expectations of trainees, tutors and employers towards this style of training.

Setting and method: PRPS modular participants based across Scotland from 2013/14 through to 2015/16 were invited to participate in a piloted semi-structured telephone interview. Interviews were recorded, transcribed verbatim and analysed using the framework approach. The analysis of key themes was verified by a second researcher independent to the study.

Main outcome measures: The views and experiences of trainees, tutors and employers towards the NHS Education for Scotland, Preregistration Pharmacist Scheme, modular programme.

Results: Four key themes were identified: relationships; preparedness; working practices and trainee attributes and motivations. The programme provided a broad experience of pharmacy practice. A practical understanding of the pharmacists' role within primary and secondary care and working with tutors and teams across multiple settings provided a more integrated and holistic approach to patient care. Tutors and employers felt this produced professionally prepared and highly employable pharmacists. Trainees highlighted that the programme informed their choice of career path, however it was demanding and suited well-motivated individuals.

Conclusion: This study indicated the positive views of modular participants towards continuation of this style of training. Future improvements were highlighted which include ensuring effective communication between tutors and supporting trainees through the transition process between placements.

Disclosure of Interest: F. Woodley Employee of: NHS Education for Scotland, R. Edwards: None Declared, G. Craig Employee of: NHS Education for Scotland, A. Power Employee of: NHS Education for Scotland.

\section{HP-PC039: Inappropriate prescribing in ambulatory elderly} patients in a geriatric clinic in Nigeria

\section{W. Akande-Sholabi ${ }^{1, *}$, O. C. Ajilore ${ }^{1}$, S. Showande ${ }^{1}$,} L. Adebusoye ${ }^{2}$

${ }^{1}$ Clinical Pharmacy and Pharmacy Administration, Faculty of Pharmacy, University of Ibadan, ${ }^{2}$ Chief Tony Anenih Geriatric Centre, University College Hospital, University of Ibadan, Ibadan, Nigeria
Background and objective: Inappropriate prescribing among the elderly is a prominent problem with substantial undesirable health consequences. In US and Europe, criteria for potentially inappropriate prescribing have been used in the past decades to identify populations at risk and target risk management strategies. However, in Nigeria little information is available about potentially inappropriate prescribing. The Beers' and STOPP (Screening Tool of Older Persons' Prescription) criteria are common tools used for screening of potentially inappropriate medications. This study aims to identify and compare potentially inappropriate prescribing in ambulatory elderly patients using the Beers' and Screening Tool of Older Person's Prescription (STOPP)/Screening Tool to Alert Right Treatment (START) criteria.

Setting and method: A 12-week retrospective study at the geriatric clinic, University College Hospital, Ibadan, Nigeria. With the aid of semi-structured questionnaires, information on the socio-demographic characteristics, lifestyle habits, drug utilisation pattern and morbidities were obtained. The Beers', STOPP and START criteria were used to identify the Potentially Inappropriate Prescribing (PIPs) and Potential Prescribing Omissions (PPOs). Drug utilization were determined using the Nigerian national essential medicine list. Data were analysed using descriptive statistics.

Main outcome measures: Identification of inappropriate prescribing and prescribing omission under the classification of Beers', STOPP and START criteria. Adherence to the Nigerian national essential medicine list.

Results: A total of 335 patients case files were reviewed, mean age was $69 \pm 0.4$ years and $219(65.4 \%)$ were females. An average of 4.2 medications per patient (total prescriptions: 4225 medicines) was found. The Beers' criteria identified potentially inappropriate prescribing (PIP) in $26.5 \%$, while STOPP criteria identified PIP in $57.1 \%$ of patients. START detected a 7.6\% Potential Prescribing Omissions (PPOs). The most prevalent disease condition was hypertension $70.1 \%$ and utilization from Nigerian national list of essential medicine was $62.2 \%$.

Conclusion: The STOPP/START criteria identified higher proportion of PIP among elderly patients compared with Beers' criteria in this study. It also detected PPOs. Prescribers should endeavour to use the criteria in order to prevent PIP and its attendant complications in the elderly. The findings in this study sensitizes us to the need to develop a national criteria for identifying potientially inapproriate prescribing in the Nigerian populations.

Disclosure of Interest: None Declared.

HP-PC040: Safer management of the 5-azacytidine administration in domiciliary care in patients with myelodysplastic syndrome

\section{Alarcón-Payer ${ }^{1, *}$, A. Jiménez Morales ${ }^{1}$, C. García Collado ${ }^{1}$,} R. García Fumero", M. Jurado Chacón ${ }^{2}$

${ }^{1}$ Servicio De Farmacia, ${ }^{2}$ Servicio De Hematología, Hospital

Universitario Virgen De Las Nieves, Granada, Spain

Background and objective: Most patients with intermediate- 2 and high-risk myelodysplastic syndrome (MDS) have a median age of 75 years and $25 \%$ of them are diagnosed beyond 80 years of age. Therefore, many of them may have great difficulty to travel to the hospital during the 7 days of duration of each cycle of 5-azacytidine treatment. The aim of this study is to analyse the safer management of the administration of 5-azacytidine in the domiciliary care and to evaluate the therapeutic adherence.

Design: 2-year prospective observational study on 30 MDS patients with a median of age of 75 years, with difficulty to travel to the day hospital to received treatment with 5-azacytidine during 7 days. The drug was prepared in the hospital pharmacy service, using the water 
reconstitution method for refrigerated injections and kept in refrigerator $\left(2-8{ }^{\circ} \mathrm{C}\right)$, resulting in both chemically and physically stable solutions for $22 \mathrm{~h}$. Once the inclusion of the patient in the study was confirmed by the haematologist, the prescribed treatment regime was informed to the pharmacy service and to the nurse to organize medication regime in the domiciliary care. The variables considered in this study were: beginning of treatment with 5-azacytidine, treatment duration, level of satisfaction of patients, treatment adherence and detected side effects.

Results: 30 MDS patients received treatment with 5-azacytidine in domiciliary care during a mean of 15 months of treatment. $75 \%$ of the patients showed great difficulty to travel to the day hospital because they required an accompanying person and 25\% did not possess supporting infrastructure. $100 \%$ of the patients were highly satisfied with the service, therapeutic adherence improved in $97 \%$ and it was detected side effects in $20 \%$ of them (neutropenia, anaemia and gastrointestinal reactions).

Conclusion: The administration of 5-azacytidine in domiciliary care in MDS older patients with difficulty travelling to the day hospital has allowed us to bring support to these patients, improving the day hospital logistics, increasing the satisfaction of the patients and its adherence to the treatment and offering a better quality healthcare.

Disclosure of Interest: None Declared.

\section{HP-PC041: Prevalence and predictors of polypharmacy} in Jordan: implications for pharmacists

\section{S. I. Al-Azzam ${ }^{1, *}$, M. Nusair ${ }^{2}$, R. Arabiat ${ }^{2}$}

${ }^{1}$ Clinical Pharmacy, Jordan University Of Science And Technology, ${ }^{2}$ Faculty of Pharmacy, Yarmouk University, Irbid, Jordan

Background and objective: Multimorbidity and therapeutic guidelines are resulting in more medication use (i.e., polypharmacy). Polypharmacy is a growing concern particularly for older patients. Using multiple medications has been associated with increased risk of adverse drug events, taking inappropriate medications, compliance issues, and increased financial burden on patients and the healthcare system. This study aims to determine the prevalence and predictors of polypharmacy in Jordan.

Setting and method: This cross-sectional study was conducted in out-patient clinics at six major hospitals in Jordan. Patients at these clinics were approached by clinical pharmacists to elicit patients' information (demographics, medical, medication and social histories, in addition to information gathered from electronic health records). Predictors of polypharmacy were evaluated using logistic regression analysis.

Main outcome measures: Major polypharmacy ( $\geq 5$ medications) and excessive polypharmacy ( $\geq 10$ medications).

Results: Data were collected from 2747 patients $(58.9 \%$ female, mean age \pm standard deviation: $57.33 \pm 13.0$ years). A total of 1156 $(42.1 \%)$ and 149 patients $(5.4 \%)$ were found to have major and excessive polypharmacy respectively with an average of 5.0 medications per patient. Significant predictors for both major and excessive polypharmacy were: socio-demographic factors (e.g., age, level of education, insurance) and medical conditions (e.g., hypertension, diabetes, asthma, hypothyroidism). Moreover, family history of ischemic heart disease and hypertension were predictors of excessive polypharmacy $(p<0.005)$.

Conclusion: Almost $50 \%$ of patients in Jordan were found to have polypharmacy. Apart from Multimorbidity as a predictor, patients' illiteracy and lack of proper education are associated with this issue. Moreover, the availability of health insurance may motivate patients to seek multiple prescribers in different clinics; the lack of national electronic health records could consequently result in duplication or unnecessary drug therapy. This study highlights the need for expanding the role of pharmacist in Jordan to include medication reviews for polypharmacy patients and recommend deprescribing when necessary.

Disclosure of Interest: None Declared.

HP-PC043: Adequacy and acceptance of the pharmacists' intervention in a tertiary hospital
A. Siles ${ }^{1, *}$, L. Vilaró ${ }^{1}$, M. Bosch ${ }^{1}$, M. Álvarez ${ }^{1}$, X. Bonafont ${ }^{1}$
${ }^{1}$ Pharmacy, Hospital Universitari Germans Trias I Pujol, Badalona, Spain

Background and objective: One of the tasks of a hospital pharmacist is the medical order's review. If a medical order is considered inappropriate, the pharmacist contacts with the physician and generates an intervention. Our objective is to evaluate the adequacy of the pharmacist interventions (PI) and their acceptance by the physician.

Design: Retrospective, unicentric and descriptive study carried out from February to March 2017 in a tertiary university hospital using computerized provider order entry (CPOE) with clinical decision support (CDS). The pharmacist responsible for every ward validated the prescriptions generated every day and reported the PI in the CPOE. PI were evaluated and classified by type of intervention and unit of hospitalization. Patients admitted in critical and emergency units were excluded, as well as the informative PI because they did not produce changes in the patient's prescription. PI were considered appropriate based on the patient's clinical situation, recommendations for use of the drug and available guidelines. Additionally, PI were considered accepted if they generated a change in the prescription. If the prescription changed as the pharmacist suggested, it scored 1, while if it changed but not as suggested, it scored 0.5. Non-accepted PI scored 0. STATA ${ }^{\circledR} 14$ was used for the statistical analysis. Percentages were calculated for qualitative variables and Chi squared to evaluate the association between them.

Results: 1629 PI were reported but only 730 PI were included in the study according to its design. $77.4 \%$ of interventions were considered adequate, and $55.82 \%$ promoted a change in the medical order. $17.4 \%$ were not accepted with a justified reason.

The units of hospitalization with better adequacy were Pediatrics and Cardiology (both $100 \%$ and $p<0.05,31$ and 41 PI respectively), where the acceptance rate was also statistically better $(100 \%$, $p<0.05$ and $81.4 \%, p<0.05$ respectively). On the other hand, the units with less adequate PI were Hepatology and Gastroenterology (63.41\% of 164 PI, $p<0.05)$ and Internal Medicine $(69.11 \%$ of 246 PI, $p<0.05)$, but only at Internal Medicine the acceptance was statistically lower $(46.95 \%, p<0.05$ for Internal Medicine unit; $49.7 \%$, $p>0.05$ for Hepatology and Gastroenterology). Oddly, Nephrology had a better adequacy rate, but its acceptance rate was statistically lower (59 PI, $91.53 \%$ of adequacy, $48.31 \%$ of acceptance, both $p<0.05)$.

According to the type of intervention, the suggestions regarding dosage adjustment and therapy discontinuation were statistically more adequate (99\% of 100 and $82.69 \%$ of 335 PI respectively, both $p<0.05$ ) but only the second type was statistically more accepted (77\%, $p<0.05)$, compared to sequential therapy interventions that were statistically less adequate $(51.09 \%$ of $184 \mathrm{PI} p<0.05)$ and less accepted $(48.64 \%, p<0.05)$.

There were no differences in other units or types of intervention. Conclusion: The adequacy degree is likely to correlate to the final PI acceptance degree as the units and types of PI with better PI's adequacy had also higher acceptance ratios, and those with worse PI's adequacy had lower acceptance ratios.

Disclosure of Interest: None Declared. 
HP-PC045: Patient safety: determine high risk factor of medication discrepancies to target patient reconciled in geriatric pathway

G. Barbier ${ }^{1, *}$, A. L. Clairet ${ }^{1}$, B. Gerard ${ }^{1}$, S. Koeberle ${ }^{2}$, J. Berthou Contreras ${ }^{1}$, V. Nerich ${ }^{1}$, S. Limat ${ }^{1}$

${ }^{1}$ Pôle pharmaceutique, ${ }^{2}$ Geriatrie, CHU Minjoz, Besancon, France

Background and objective: In France, national surveys have provided quantitative baseline data on drug iatrogenicity. Forty-seven percent of serious adverse events are related to drug. The high 5's showed that medication reconciliation is a way to detect and correct medication errors between usual and hospital prescription. Despite technical and human resource, targeting patient at high risk of medication errors seems essential to greater relevance and efficiency.

The objective of our study was to determine whether risk factors were associated with medication errors in reconciled patients.

Design: We conducted a prospective study in a geriatric ward at the hospital. All patients admitted and reconciled from 1st December 2015 to 30th June 2017 were included. Two groups of patient were defined. First group included patients with unintentional discrepancies at admission (UID) and the second group without UID (nUID). Several potential risk factors for prescribing medication error occurrence were searched for. They include: patient characteristics (age, sex, renal function); health (antecedent, reason for admission) and prescription characteristics (number and type of drugs). A statistical analysis of the potential risk factors was conducted.

Results: A total of 658 patients were included in the study (mean age, 86 years). Overall, 161 (24.5\%) patients had at least one unintentional discrepancy. Of the 161 patients with UID, 10 had at least one hydroelectrolyte disorder on admission $(6.2 \% ; p=0.01)$ considered to be a significant risk factor of UID. Digestive symptoms were identified on 19 patients with UID $(11.8 \% ; p=0.04)$ versus 32 patients with nUID $(6.4 \%)$ as a potential risk factor. Instead, patient characteristics were not increased risk of UID. Polymedication were involved in significant risk factor of medication errors $(p=0.0084)$.

In the multivariate logistic regression model, hydro-electrolyte disorder $(\mathrm{OR}=3.223 ; 95 \%$ IC $1.308-7.943 ; p=0.0110)$ and presences of at least 9 drugs on admission (OR $=1.622$; 95\% IC 1.132-2.325; $p=0.0085$ ) were associated with a higher proportion of patients with UID.

Conclusion: These findings differ from other studies in the literature. The method has several limitations. The risk factors researched were based on studies in general population, which is prone to selection bias in our elderly population.

Risk management, anticholinergic drug scale or other indicators must be explored to target patient reconciled.

Disclosure of Interest: None Declared.

\section{HP-PC048: Medication preparation by pharmacy technicians: a pilot study}

D. Bornand ${ }^{1, *}$, S. Krauss ${ }^{2}$, M. Stalder ${ }^{1}$, F. Yasar ${ }^{1}$, M. Bahor ${ }^{1}$, N. Waeber ${ }^{1}$, C. R. Meier ${ }^{1}$

${ }^{1}$ Hospital Pharmacy, ${ }^{2}$ Surgery Ward, University Hospital Basel, Basel, Switzerland

Background and objective: The medication dispensation process includes preparation, documentation and administration of medication. It is predominantly a nursing activity and has an estimated risk for medication errors up to $30 \%$. Organizational and structural factors may be reasons for medication errors. The " 7 rights" ensure a secure and correct preparation and administration process. It was the aim of the study to include pharmacy technicians in the preparation process to optimize the process, minimize disruptions, and introduce a double check for all medications.

Setting and method: Three pharmacy technicians prepared and documented alternately the medication at a surgery ward for 4 weeks from Monday to Friday for all patients. They substituted or ordered the medication, which was not available in the dispensary. Afterwards nurses controlled the medication before application.

Main outcome measures: We assessed the time and disruptions in relation to the patient and medication counts for pharmacy technicians. We also quantified the express orders, discrepancies between the medication and prescription, and the level of satisfaction in both teams.

Results: After an introduction of 2 weeks to get used to the new task, pharmacy technicians prepared drugs for a median of 28 patients per day with 147 medications and 237 units, requiring on average 4 min per patient for preparation and documentation. The introduction of pharmacy technicians reduced emergency orders by half. Nurses and pharmacy technicians were highly satisfied. There was no reduction in discrepancies during the short duration of the pilot study.

Conclusion: Pharmacy technicians prepared the medication in a similar time as the nurses after an introduction to the preparation process. We optimized the process and introduced an overall improvement to patient safety. Nurses and pharmacy technicians provided positive feedback on this new process.

Disclosure of Interest: None Declared.

HP-PC049: Prevalence of inappropriate prescribing in elderly patients in French geriatric acute ward

L. Bossy ${ }^{1, *}$, J. Bonhomme ${ }^{1}$, A. Vignoles ${ }^{2}$, T. Gilbert ${ }^{2}$, C. Rioufol ${ }^{1}$, M. Bonnefoy ${ }^{2}$, S. Parat ${ }^{1}$

${ }^{1}$ Département de Pharmacie Clinique et Médicament, ${ }^{2}$ Service de Court Séjour Gériatrique, Centre Hospitalier Lyon Sud, Lyon, France

Background and objective: Elderly people have multiple comorbidities and, as a consequence, multiple drugs are prescribed. Inappropriate prescribing due to polypharmacy is a well-known issue in elderly patients and it increases adverse drug events. Since November 2016, a clinical pharmacist team was present in the ward to reconcile and review medications at admission.

The purpose of the study is to evaluate the prevalence of inappropriate prescription in elderly patients hospitalized in university acute geriatric ward according to STOPP/START criteria V2 ${ }^{1}$.

Design: A prospective observational study was performed during 3 months from January to March 2017. All reconciled patients at admission were included. A clinical pharmacist resident reviewed all medications, diseases, comorbidities and laboratory data, and determined Charlson Comorbidities Index for each patient. Inappropriate prescribing (IP) included Potentially Inappropriate Medication (PIM) and Potentially Prescription Omission (PPO) was identified according to French version of STOPP/START criteria V2.

Results: For the study, 108 patients were included. Mean age was $84.6 \pm 6.5$ years and mean number of medications per patient was $8.5 \pm 3.8$. Out of 108 patients, $76.9 \%$ have at least one STOPP criteria and $56.5 \%$ have at least one START criteria. According to Charlson Comorbidities Index, $36.1 \%$ patients have at least 3 comorbidities. A total of 318 IP (225 PIM and 93 PPO) was detected. The average number of IP per patient is $3.8 \pm 2.3$.

Nervous system/psychoactive medicines and cardiovascular system medicines represent respectively $19.5 \%$ and $14.8 \%$ of inappropriate prescribing. Three STOPP/START criteria gather 109 IP $(34.3 \%)$ : over-prescription (STOPP A1), prescription of benzodiazepines for more than 4 weeks (STOPP D5), and prescription of benzodiazepine which predictably increases the risk of falling in the elderly patients (STOPP K1). 
Furthermore, patients with high Charlson Comorbidities Index $(\mathrm{CCI} \geq 3)$ have statistically more inappropriate prescriptions $(p=0.659)$.

Finally, there is a positive correlation between the Charlson Comorbidities Index and the number of STOPP/START criteria $(p=0.700)$.

Conclusion: There is a high prevalence of potentially inappropriate medications in our elderly multimorbidity patients. Using STOPP/ START criteria would have two major benefits: to prevent adverse drugs events, if it is used in a structured clinical pharmacist medication review, and to train residents at the identification of inappropriate prescribing.

\section{Reference}

1. Lang PO, Boland B, Dalleur O. L'adaptation en franc, ais des critères STOPP/START.v2.

Disclosure of Interest: None Declared.

HP-PC050: Implementation and evaluation of pharmaceutical care in the management of oncology patients in a small size peripheral hospital

\section{O. Polinard ${ }^{1}$, M. Bourton ${ }^{1, *}$, M. Mattens ${ }^{1}$}

${ }^{1}$ Pharmacy, St. Nikolaus-Hospital, Eupen, Belgium

Background and objective: Given the toxicity of cancer treatments, patient frailty, the variety of existing treatments and those under development, as well as polymedication, oncology patients are exposed to many drug-related problems (DRP). Clinical pharmacists have an important role to play in this field and therefore we implemented and evaluated new clinical pharmacy services at our hospital's outpatient oncology clinic.

Design: A 6-month study in a 200-bed peripheral hospital with an intervention group of patients starting cancer treatment and a control group of patients already on cancer treatment. Patients were counselled by the clinical pharmacist regarding their current cancer treatment and concomitant drug therapy, with a focus on DRP, medication discrepancies and adherence. In the intervention group, patients were seen at the beginning of the first treatment cycle and had at least one follow-up consultation. In the control group, patients only had one pharmaceutical consultation. Pharmaceutical interventions were subsequently suggested to the oncologists (as part of a pharmaceutical consultation report published in the patient's electronic medical record) and to patients. Staff satisfaction and collaboration with community pharmacists were also investigated.

Results: 63 patients were enrolled and 103 pharmaceutical consultations took place. When collecting medication histories, a total of 766 medication lines were recorded, out of which 293 medication discrepancies were identified. A total of 233 DRP were identified, mainly (potential) drug interactions $(51 \%)$. After pharmaceutical analysis of these DRP, 224 interventions were suggested to the oncologists and 54 to the patients. 37 adverse drug reaction reports were sent to the national authorities. Overall satisfaction was rated as excellent by the oncology team and community pharmacists welcomed being involved in their patients' cancer treatment.

When comparing intervention $(n=28)$ and control groups $(n=28)$, statistical analysis showed that the number of DRP in the intervention group was significantly lower. Adherence was better in the intervention group, but the difference between both groups was not statistically significant.

After this evaluation, the service was modified in order to optimize resources. Currently, only patients on oral cancer drugs are included, because a contact point was lacking for this patient group, as medicines are dispensed by the hospital pharmacy. They are now counselled by the pharmacist when beginning a new treatment. Two weeks later an oncology nurse checks for adverse drug reactions. Afterwards patients have a monthly consultation on top of their medical appointments, alternately with a pharmacist or an oncology nurse.

Conclusion: This study shows that our newly implemented clinical pharmacy service adds value for both the patient and the multidisciplinary oncology team. An evaluation of the optimized service is planned in the near future.

Disclosure of Interest: None Declared.

HP-PC051: Outcome description of pharmacists interventions in a University Teaching Hospital in Southern Ireland

N. Daly ${ }^{1}$, A. Harnett ${ }^{1}$, S. Byrne ${ }^{2, *}$

${ }^{1}$ Pharmacy Department, University Hospital Limerick, Limerick, ${ }^{2}$ School of Pharmacy, University College Cork, Cork, Ireland

Background and objective: Pharmacists, the experts in medication have the ability to improve the quality of care delivered to patients. Prescribing errors have been identified as a significant cause for concern to patient safety across many healthcare providers and pharmacist have the skills and opportunity to intervene and prevent the risk of unnecessary harm. Medication reconciliation (MR) is 'the process of creating and maintaining the most accurate list possible of all medications a person is taking including drug name, dosage, frequency and route'. It is internationally recognised that where a patient's care is transferred to a new service provider, MR should be completed. The aim of the study was to investigate the expected harm that may occur to patient's wellbeing if a pharmacist had not intervened.

Setting and method: This study was undertaken at a University Affiliated hospital with 428 in-patient beds. A data collection form was designed and piloted for the purpose of this study. Data collection was carried out across six medical wards (including an Intensive Care Unit and a High Dependency Unit. Interventions) by a group of pharmacists over a 14-day period between 27th Feb 2017 and 16th Mar 2017. Inclusion criteria; all patients admitted to one of the six medical wards from Mon to Fri ( $9 \mathrm{am}-5 \mathrm{pm})$ who were reviewed by a pharmacist. Exclusion criteria; included patients not reviewed by a pharmacist, those admitted to a ward outwit the study and those admitted over the weekend. A panel of experts categorised the interventions into high, medium, low, very low and no risk of harm. The panel initially graded the interventions separately and then came together to discuss interventions which they did not agree upon in order to reach a consensus. Local ethics approval was sought and gained for this study.

Main outcome measures: Categorisation of pharmacsits interventions.

Results: In total 504 patients were reviewed, 275 (56\%) patients were in receipt of an intervention. The study demonstrated that the majority of pharmacist interventions may have prevented risk of harm to patient safety $(82.15 \%)$. Pharmacist interventions completed upon admission prevented a higher than expected quantity of high risk situations occurring. This result was statistical significant $(p=0.04)$. The results also demonstrated that by completing MR post admission pharmacists' interventions were more likely to prevent high risk of harm to patients. Pharmacists targeted high risk and high alert medicines, that when used inappropriately, may lead to catastrophic events for patient involved. The results depict that the admission point of care is a critical point of patient contact for pharmacists. Meaningful, worthwhile interventions are undertaken by pharmacists here. 
Conclusion: Interventions made by pharmacists in our hospital are paramount in ensuring medication safety is maintained. The admission stage of patient care is an effective time for pharmacists to made worthwhile interventions. MR significantly improves the quality of patient care provided.

Disclosure of Interest: None Declared.

HP-PC052: Evaluation of the etiology and resistance of the bacterial pathogens involved in bloodstream infections

S. Hancu ${ }^{1, *}$, O. Zlatian $^{2}$, O. Cristea ${ }^{2}$, L. Hancu ${ }^{3}$, A. T. Balasoiu ${ }^{2}$, M. Bacanoiu ${ }^{4}$, A. F. Rosu', A. O. Docea ${ }^{2}$, D. Calina ${ }^{2}$

${ }^{1}$ Fundeni Hospital, Bucharest, ${ }^{2}$ University of Medicine and Pharmacy, Craiova, ${ }^{3}$ University of Medicine and Pharmacy "Carol Davila”, Bucharest, ${ }^{4}$ Clinical Emergency Hospital, Craiova, Romania, ${ }^{5}$ Hospital Garcia de Orta, Lisbon, Portugal

Background and objective: Invasive infections are the most severe infections known because the germs are blood borne and have the ability to inoculate multiple sites, leading to sepsis that often leads in turn to septic shock with a high mortality rate.

The present study aims to assess the prevalence rate of invasive infections in hospital environment, to enumerate their etiology in a specific hospital and to establish antibiotic resistance profiles needed for treatment adjustment.

Setting and method: We collected blood cultures from 505 patients (216 women and 289 men) hospitalized in Clinical Emergency Hospital of Craiova, Romania, between 01.09.2016 and 31.07.2017. The patients were hospitalized in Intensive Care Unit (ICU) - 272 patients, surgical wards (General Surgery, Plastic Surgery, Orthopedic, Pediatric Surgery, Ophthalmology, Neurosurgery, Urology, Gynecology) - 47 patients, medical wards (Internal Medicine, Cardiology, Diabetes, Nephrology, Neurology) 129 patients, pediatric wards 45 patients and oncological wards- 12 patients.

Main outcome measures: A total of 974 blood cultures were collected. We have used Bactalert 3D and Vitek 2 Compact systems (Biomerieux, USA) to analyse the samples.

Results: The prevalence rate of invasive infections was $27.72 \%$. We isolated 170 bacterial strains, the most frequent were Staphylococcus aureus (63 strains-37.06\%), Klebsiella spp. (27 strains-15.88\%), Coagulase Negative Staphylococci (CoNS) (18 strains-10.59\%), Enterococcus spp. (17 strains-10.00\%), Escherichia coli (12 strains-7.06\%), Streptococcus spp. (10 strains-5.88\%) and other bacterial species.

MRSA global prevalence was $36.51 \%$ from SA strains or $2.36 \%$ from total samples analyzed. There were significant differences in MRSA prevalence between adults and children $(37.50 \%$ vs. $28.57 \%$, $p=0.047)$ and between ICU and non-ICU patients $(42.42 \%$ vs. $30.00 \%, p=0.018$ ).

The multivariate analysis of the risk of acquisition an invasive infection with any germ demonstrated a significant effect of admission into Plastic surgery $(\mathrm{OR}=1.444, p=0.082)$ and Cardiology $(\mathrm{OR}=2.313, p=0.001)$. The multivariate analysis of the risk of acquisition an invasive infection with MRSA demonstrated a significant effect of male sex $(\mathrm{OR}=1.684, p=0.080)$, age over 50 years $(\mathrm{OR}=2.134, p=0.050)$, admission into $\mathrm{ICU}$ unit $(\mathrm{OR}=1.518$, $p=0.058)$, Plastic surgery $(\mathrm{OR}=3.299, p=0.044)$, Medical wards $(\mathrm{OR}=0.278, p=0.075)$ and Cardiology $(\mathrm{OR}=2.088, p=0.009)$.

Conclusion: The most common pathogen from blood cultures was Staphylococcus aureus, which presented instances of multiple antibiotic resistance, making difficult the management of these invasive infections.

Disclosure of Interest: None Declared.
HP-PC053: Impact of an Antimicrobial Stewardship Program on an internal medicine department

S. E. Campbell Davies ${ }^{1, *}$, V. Di Maggio ${ }^{1}$, E. Galfrascoli ${ }^{1}$, S. Bollani ${ }^{2}$, A. Zambelli ${ }^{3}$, S. Grosso ${ }^{4}$, G. Muserra ${ }^{1}$

${ }^{1}$ Pharmacy, ${ }^{2}$ Internal Medicine, ${ }^{3}$ Infectious Diseases, ${ }^{4}$ Microbiology, Fatebenefratelli e Oftalmico Hospital - ASST Fatebenefratelli Sacco, Milan, Italy

Background and objective: According to the World Health Organization (WHO) the most effective strategy against antibioticresistance, which increases hospitalisation and mortality, is the implementation of Antimicrobial Stewardship Programs (ASPs). Since 2015, an ASP in the centre has been carried out, where a multidisciplinary team consisting of Pharmacists, Clinicians, Microbiologists, Infectious Disease Specialists and Nurses work together to improve the appropriate use of antibiotics. The objective is to evaluate the impact of the ASP on the use of antibiotics and on bacterial resistance.

Setting and method: Data from three 3-months periods were extracted from medical records in an internal medicine department and collected in a database: T0 (December 2016-February 2017), T1 (April-June 2017), T2 (August-October 2017). During these periods two audits took place (March, July 2017) and monthly clinical reports were produced.

Main outcome measures: Use of antibiotics expressed in defined daily dose (DDD), number of treatment lines per patient, number of resistant microorganisms, number of nosocomial infections, duration of hospitalisation.

Results: A total of 444 patients were monitored: 122 (T0), 157 (T1) and 165 (T2). At T0, 78\% of patients were treated with at least 1 antibiotic, at $\mathrm{T} 167 \%$ and at $\mathrm{T} 264 \%$. A reduction of antibiotic prescriptions was shown after the two audits (14\%) however ceftriaxone was the most prescribed molecule over all periods. Out of 444, 216 medical records were analysed: 65 (T0), 87 (T1) and 64 (T2). An important reduction of meropenem prescriptions was obtained (DDD - 37\%). Consequently, Klebsiella Pneumoniae Carbapenamase-producing K. pneumoniae (KPC-Kp) cases reduced by $80 \%$. The number of targeted prescriptions for meropenem increased by $33 \%$ demonstrated a better use of this drug. After the audits, the number of treatment lines decreased $(-38 \%)$, suggesting an improvement in prescription quality. Furthermore, between $\mathrm{T} 0$ and $\mathrm{T} 2$ a decrease of nosocomial infections (T0: $22 / 65, \mathrm{~T} 2: 9 / 64 ;-20 \%$ ), hospital stay (T0: $27 \pm 17, \mathrm{~T} 2: 14 \pm 9 ;-52 \%)$ and antibiotic consumption (T0: $€ 7198, \mathrm{~T} 2$ : €5915 euro; - 18\%) was obtained.

Conclusion: By using tools such as audits and multidisciplinary team reports, the ASP has improved clinicians' awareness about the responsible use of antibiotics contributing to patient's quality of healthcare though a positive impact on bacterial resistance, nosocomial infections and hospital stay.

Disclosure of Interest: None Declared.

HP-PC054: Antihypertensive drugs in elderly patients: pharmacists' interventions in medication reconciliation

\section{S. E. Campbell Davies ${ }^{1, *}$, S. Nobili ${ }^{1}$, E. Galfrascoli ${ }^{1}$, G. Muserra ${ }^{1}$}

${ }^{1}$ Pharmacy, Asst Fatebenefratelli Sacco - Fatebenefratelli E Oftlamico Hospital, Milan, Italy

Background and objective: Hypertension is a known risk factor for cardiac, cerebrovascular and renal diseases. Treatment with antihypertensive drugs can reduce this risk even in the elderly, however, several studies have suggested that there is an increased risk of falls in elderly patients taking these medications. Medication reconciliation 
can be an important tool to review patients' treatments in order to improve appropriate prescribing and potentially reduce the risk of falls. The objective of the study is to assess pharmacists' interventions (PIs) on elderly patients' treatment with antihypertensive drugs.

Setting and method: This prospective study was performed between November 2017 and March 2018 in two internal medicine wards. Patients included were age 65 or over taking five or more medications during hospitalisation. Data were collected through patient's records and included: age, sex, comorbidities, reason for admission, number and type of drugs. MR was conducted and drug related problems (DRPs) were identified by the pharmacist using several sources (Micromedex, drug databases, local and international guidelines).

Main outcome measures: Difference in the number of DRPs on admission and at discharge and PI acceptance rate.

Results: Forty-four ( $40.9 \%$ male, mean age $82.6 \pm 7.4)$ out of 50 screened patients presented hypertension $(88 \%)$ and were treated with at least one antihypertensive drug on admission (mean number of drugs per patient on admission: $8.3 \pm 3.3$ ). Fifteen patients (34\%) were admitted for falls and $40 \%$ were being treated with an association of 4 antihypertensive medicines (40\%). On admission, 115 antihypertensive drug prescriptions were recorded belonging to 5 different classes (ATC $\mathrm{C} 02$ antihypertensive, $\mathrm{C} 03$ diuretics, $\mathrm{C} 07$ beta blocking agents, C08 calcium channel blocking agents, C09 agents acting on the renin-angiotensin system). The number of DRPs connected to antihypertensive treatments on admission was 55 out of 202 total DRPs considering all medication taken by the patient. After the pharmacists' intervention, the number of antihypertensive prescriptions at discharge reduced to 95 while the number of DRPs reduced to $21(-62 \%)$. The number of recommendations that the pharmacist discussed with the clinician about antihypertensive drugs was 82 and the overall acceptance rate of PIs was $80 \%$ (43/52 drug interruptions, 4/5 drug substitutions, 15/21 dose adjustments, 4/4 drug introductions).

Conclusion: This study has demonstrated that the pharmacist has an important role in reviewing patients' prescriptions. Improving appropriate prescribing of antihypertensive therapy in elderly patients could reduce the risk of falls and, consequently, the number of hospitalisations and the healthcare costs. Further studies are needed to assess the rate of rehospitalisation of these patients and their quality of life.

Disclosure of Interest: None Declared.

\section{HP-PC055: A case of intertriginous and flexural exanthema (sdrife) caused by amoxicillin}

B. Cancela-Díez ${ }^{1, *}$, S. Sadyrbaeva-Dolgova ${ }^{1}$, D. López-Delgado ${ }^{2}$, R. Alvarez ${ }^{1}$, R. Ruiz-Villaverde ${ }^{2}$

${ }^{1}$ Pharmacy, ${ }^{2}$ Dermatology, Complejo Hospitalario Universitario de Granada, Granada, Spain

Background and objective: Baboon syndrome (BS) is a systemic contact dermatitis that occurs after systemic exposure to an allergen in individuals previously sensitised to it topically. Hauserman et al. proposed the acronym SDRIFE "symmetric drug related intertriginous and flexural exathema" for cases of BS in which previous cutaneous sensitisation could not be demonstrated and were provoked by the systemic administration of medications.

The aim is to describe a case of SDRIFE caused by amoxicillin and to describe the main characteristics for the diagnosis of SIDFRE as oppose to other skin rushes.

Design: 50-year-old patient with a symmetrical inguinal eruption and subsequently on the dorsum of both feet. Symptoms appeared $4 \mathrm{~h}$ after provocation test with amoxicillin. The patient was not undergoing treatment with any other medication.
The physical examination showed macular lesions, confluent, some pinpoint, purplish in colour, symmetrically positioned on the back of the feet, ankles and groin. With regard to the complementary tests, the analytics were within normal range and the patient did not show systemic signs or symptoms. Parvovirus B19 had negative serology. The patient was admitted to control the progress of exanthema and coagulation, the use of B lactam antibiotics was prohibited and $20 \mathrm{mg} / 24 \mathrm{~h}$ treatment with bilastina was applied before completing the 20-day treatment, with favourable progress. In addition, prior to the patient's consent, a provocation test was performed with amoxicillin, with positive result for the second time.

Results: The diagnosis was SIDFRE because the patient presented the following criteria: systemic exposure to a drug for the first time or repeatedly; erythema demarcated in gluteal/perianal area and/or V-shaped erythema in inguinal/perigenital area; at least one other intertriginous or bending area being affected;condition of these areas symmetrically developed and an absence of systemic signs and symptoms. Parvovirus B19 infection may present the flexural erythema characteristics of SIDFRE but it was ruled out for presenting negative serology.

Conclusion: SDRIFE is rare and presents clinical features similar to other dermatologic disorders, so it is important for the clinician to recognise these characteristics and their possible causes, in order to establish an adequate diagnosis.

Disclosure of Interest: None Declared.

HP-PC056: Dabigatran $150 \mathrm{mg}$ vs dabigatran $110 \mathrm{mg}$ in patients older than 80 years old

I. Casas Hidalgo', M. A. Garcia Lirola ${ }^{2}$, R. Alvarez Sanchez ${ }^{1, *}$, J. Cabeza Barrera ${ }^{1}$

${ }^{1}$ Farmacia Hospitalaria, Hospital Universitario San Cecilio, ${ }^{2}$ Atención Primaria, Distrito Metropolitano, Granada, Spain

Background and objective: Atrial fibrillation (AF) is the most frequent chronic cardiac arrhythmia. It is estimated that it affects $1-2 \%$ of the population. Its prevalence increases with age, therefore it is estimated that $8.5 \%$ of the Spanish population over 60 years suffers this patology.

Dabigatran is a new oral anticoagulant (NACO), which is metabolized primarily at the renal level, indicated among other pathologies for the prevention of stroke and systemic embolism in patients with nonvalvular $\mathrm{AF}$ with some risk factors.

The established daily dose of this drug is $150 \mathrm{mg}$ every $12 \mathrm{~h}$. However, in patients older than 80 years, the recommended dose is $110 \mathrm{mg}$ every $12 \mathrm{~h}$ since according to the scientific literature no statistically significant differences are observed in terms of stroke reduction and mortality between both regimens.

The objective of this study is to evaluate the treatment with Dabigatran in patients older than 80 years who present with non-valvular AF.

Design: Descriptive observational study that evaluates the prescribed dose of Dabigatran in patients with non-valvular AF older than 80 years from August to October 2017. The variables studied, obtained from clinical histories through the Diraya and MicroStrategy computer applications, were: sex, age, renal clearance, posology and adverse reactions of treatment with Dabigatran.

Results: Dabigatran was administered to 41 patients. $81 \%$ of them were women. The mean age of these patients was $83 \pm 4.40$ years. The mean value of the renal clearance was $0.87 \pm 0.23 \mathrm{mg} / \mathrm{dl}$. Of all patients treated with Dabigatran, 63\% (26/41) were treated with Dabigatran $150 \mathrm{mg}$ every $12 \mathrm{~h}$; while 37\% (15/41) had prescribed Dabigatran $110 \mathrm{mg}$ every $12 \mathrm{~h}$. Regarding the adverse effects associated with the treatment with the NACO studied, only one patient developed rectal bleeding. 
Conclusion: Our study shows that the majority of patients older than 80 years $(63 \%)$ are being treated with Dabigatran $150 \mathrm{mg}$. The only patient who presented adverse reactions associated with this treatment (rectal bleeding) was being treated with this dose. It confirms, as stated in the scientific literature, that there is an increased risk of gastrointestinal bleeding with the dose of $150 \mathrm{mg}$ compared to that of $110 \mathrm{mg}$.

Pharmacists can play a very important role in preventing the toxicity of this NACO (without increasing the risk of stroke/mortality) by generating alerts addressed to prescribing doctors with a view to prescribing doses of $110 \mathrm{mg}$.

Disclosure of Interest: None Declared.

HP-PC057: Orodispersible films as a paediatric dosage form: survey of caregivers' acceptability

S. Y. Chan ${ }^{1, *}$, W. C. Foo ${ }^{1}$, A. X. Lim ${ }^{1}$, X. Y. Chan ${ }^{1}$, Y. P. Koh ${ }^{1}$, N. J. Y. Phua ${ }^{1}$, H. H. Cheong ${ }^{2}$

${ }^{1}$ Pharmacy, National University of Singapore, ${ }^{2}$ Pharmacy, KK

Women and Children's Hospital, Singapore, Singapore

Background and objective: Conventional solid dosage forms fail to meet the needs of those who are unable to swallow tablets or capsules while alternative liquid medicines pose dose accuracy and other administration problems. Orodispersible film (ODF) is a promising alternative. However, few ODFs are commercially available and acceptability of ODFs has not been established in Singapore. Thus a survey of caregivers' acceptability of ODFs for their children was conducted.

To determine caregivers' perceptions towards paediatric dosage forms and their acceptability of ODFs.

Design: An IRB-approved interviewer-administered survey was carried out at a hospital outpatient pharmacy to determine caregivers' acceptability of ODFs.

The survey was conducted at KK Women's and Children's Hospital Outpatient Pharmacy.

Results: Of the 223 caregivers who completed the survey, 59\% ranked palatability of medication as their top concern and $88 \%$ were willing to administer ODFs to their children. In addition, $40 \%$ of these caregivers had to manipulate the dosage forms before administering the medication to their children. They encountered difficulties such as problems crushing the tablet or mixing the resultant powder with liquids or food. This may have further implications on palatability and integrity of the medication.

Conclusion: Our results showed that caregivers were open to the idea of administering ODFs to their children provided they are pleasant tasting and palatable.

Disclosure of Interest: None Declared.

\section{HP-PC058: Development of a high-alert medication thesaurus adapted to French hospitals}

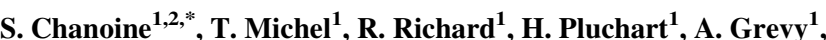
E. Brudieu $^{1}$, F. Reymond ${ }^{1}$, B. Allenet ${ }^{1,2,3}$, P. Bedouch ${ }^{1,2,3}$

${ }^{1}$ Pharmacy Department, Grenoble Alpes University Hospital, ${ }^{2}$ School of Pharmacy, Grenoble Alpes University, ${ }^{3}$ ThEMAS, CNRS, TIMCIMAG UMR 5525, Grenoble Alpes University, Grenoble, France

Background and objective: High-alert medications represent a major concern, particularly during hospitalization because of the high-risk of iatrogenic effects. Some lists of high-alert medications have been proposed but none is adapted to the French health system and none provides ranking system integrating criticality level and clinical context. We aimed at elaborating a high-alert medication thesaurus adapted to French hospitals.

Design: Based on the national/international literature and clinical experience, 9 expert multidisciplinary groups (including pharmacists, physicians, pharmacovigilance experts) selected high-alert medications according to their specific fields. For each medication, they identified the risks encountered in its use and determined by consensus the related-pharmaceutical interventions to be implemented to limit each risk. The risk level was determined for each medication based on the FMECA method (Failure Modes, Effects and Criticality Analysis), which allows determining medication crude criticality (and so the criticality rank from 1 "limited risk" to 25 "highest risk") and residual criticality according to the methods to prevent the failure implemented in each clinical environment.

Results: Among the medications (i.e. different international nonproprietary name) commercialized in France, 947 of them were identified at high-risk from the literature. Among the three multidisciplinary teams that get together (immunology, anesthesia/intensive care and infection groups), 301 medications were analyzed and 179 were defined as high-alert medications by the experts (including withdrawals and additions from the predefined list): 17, 73 and 89 in the immunology, anesthesia/intensive care and infection groups, respectively. Among each group, 243, 438 and 119 risks were identified leading to 179,452 and 127 different pharmaceutical interventions, respectively. For example, in the anesthesia/intensive care group, the main risk was drug-drug interaction $(71 \%)$ and the main pharmaceutical intervention was therapeutic monitoring $(56 \%)$. The crude and residual criticality is being determined by consensus in this group.

Conclusion: In a constrained economic context, this innovative approach offers the opportunity to improve the therapeutic management of hospitalized patients by identifying and ranking medication prescriptions according to their risk, leading to prioritize the validation of prescriptions by the pharmacist in French hospitals. Multidisciplinary groups continue developing the thesaurus and an automatic detection system that ranks medication prescriptions by priority according to the high-alert medication thesaurus is being developed.

Disclosure of Interest: None Declared.

\section{HP-PC059: Safety and regimen evaluation of methylphenidate} for brain Injury patients

\section{P.-C. Chen ${ }^{1, *}$, C.-T. Tseng ${ }^{1}$, L.-C. C. Chen ${ }^{1}$, K.-H. Yang ${ }^{1}$}

${ }^{1}$ pharmacy, Taipei City Hospital, Taipei, Taiwan, Province of China

Background and objective: U.S. Food and Drug Administration (FDA) approved indication for methylphenidate are attention deficit hyperactivity disorder (such as ADHD) and narcolepsy. Methylphenidate is a mild central nervous system stimulant that activates the brainstem arousal system, subcortical structures, and cortex including the thalamus. Although FDA proved the indications above, there were some studies implied that methylphenidate may be useful for traumatic or nontraumatic brain injury patients. This study aimed to analyze the use of methylphenidate in brain injury patients in a teaching hospital in Taiwan.

Setting and method: A retrospective review was performed in a teaching hospital in Taipei Taiwan between January 2017 and March 2018. We analyzed the diagnosis, dosage, dosage form, frequency and duration of methylphenidate for brain injury patients. We collected the lab data of these injury patients to see whether there were common and serious adverse effects of receiving methylphenidate (adverse effects information were from electronic database Micromedex).

Main outcome measures: Diagnosis, dosage, dosage form, frequency and duration of methylphenidate for brain injury patients' data 
were collected. Follow up the common and serious adverse effects of methylphenidate within these injury patients.

Results: A total of 42 patients were prescribed methylphenidate during January 2017 and March 2018. There were 37 patients were diagnosed with attention-related diseases (ICD-10-CM code ranged from F25.9 to F95.2). Five of them were diagnosed brain injury related diseases including other nontraumatic subarachnoid hemorrhages; nontraumatic intracerebral hemorrhage in hemisphere, cortical; cerebral infarction due to thrombosis of right middle cerebral artery; traumatic subdural hemorrhage without loss of consciousness, subsequent encounter. The ages of the five patients were $42,45,52$, 53 and 78. The methylphenidate regimen was $10 \mathrm{mg}$ for once daily or twice daily. Numbers of days receiving methylphenidate were 7,28 , 56, 116 and 196 days, respectively. The studies about methylphenidate for brain injury patients indicated that the dose may start low $(2.5 \mathrm{mg} /$ day) to maximum dose $60 \mathrm{mg} /$ day. The dose and frequency of the drug were appropriate. One of the patients had the transient abnormal liver function, the ALT elevated from 21 to 72 (abnormal) and back to 19 (normal) in 5 months.

Conclusion: It is pharmacists' duty to monitor patient's safety not to mention the off-label use of some drugs. Through this study, we found that the use of methylphenidate was considered appropriate yet one patient had a transient abnormal liver function. More studies are needed in the use of methylphenidate for brain injury to find the appropriate adult regimen.

Disclosure of Interest: None Declared.

HP-PC060: Impact of clinical pharmacy on the re-hospitalization rate in a geriatric emergency unit

\author{
A. Clementz ${ }^{1, *}$, J. Jost ${ }^{1}$, A. Lacour ${ }^{2}$, A. Tchalla ${ }^{2}$, \\ V. Ratsimbazafy ${ }^{1}$ \\ ${ }^{1}$ Pharmacy, ${ }^{2}$ Geriatric Medicine, Limoges Teaching Hospital, \\ Limoges, France
}

Background and objective: Elderly frail are frequently exposed to polypharmacy and inappropriate prescriptions. Iatrogenic issue is responsible for $20 \%$ of their hospitalizations and falls are one of major problem in result, while $25 \%$ might be avoided. Clinical pharmacy has already shown an interest in reducing polypharmacy and medication complexity.

Objective: to evaluate the impact of pharmacist-led interventions at the geriatric emergency department (g-ED) on patient re-hospitalization rate after discharge.

Design: Retrospective study in a referral hospital, on a period of 7 weeks.

Primary endpoint was re-hospitalization rate within the $72 \mathrm{~h}$ and 30 days at the ED between a group of patients followed by a pharmacist (FPH) who benefited from clinical pharmacy activities (medication reconciliation, pharmaceutical analysis, town-hospital network) inside the g-ED unit of ED and another group of patients without follow-up (wFPH) by a pharmacist.

Evolution of Medication Complexity Regimen Index (MRCI) during the hospitalization was assessed.

Inclusion criteria: patients with an age of at least 75 years, admitted for fall at the g-ED, more than two diseases and two medications.

Results: A total of 100 patients were included (34 male, $76-108$ years), 48 patients in FPH group and 52 patients in wFPH group. No significant differences have been observed between the two groups concerning the variables sex, origin place, medication number, drugs causing falls number, patients orientation, time spent at the g-ED. The mean age of FPH group was older (89 vs $87, p<0.008$ ).
No re-hospitalization within the $72 \mathrm{~h}$ after discharge has been observed in FPH group compare to four in the wFPH group $(p=0.1)$.

Five re-hospitalizations after 30 days have been observed in the FPH group compare to seven in the wFPH group $(p=0.76)$. A reduction of 2.3 points on the MRCI score was observed in the FPH group.

Conclusion: Clinical pharmacists help to decrease prescription complexity. We found no effect on the re-hospitalization rate at $72 \mathrm{~h}$ and at 30 days probably due to a lack of power of the study, however positive trends were observed. The patient recruitment is still ongoing to improve the study power and to specify results.

Disclosure of Interest: None Declared.

HP-PC061: Analysis of pharmacotherapeutic interventions performed in a hematology unit

A. Corral-Alaejos ${ }^{1, *}$, E. Sobejano-Fuertes ${ }^{2}$, V. Higuero-Saavedra ${ }^{2}$, L. Rodriguez-Cajaraville ${ }^{1}$, R. Fernandez-Caballero', A. M. Moreno-Gomez ${ }^{1}$, M. J. Otero ${ }^{1}$

${ }^{1}$ Pharmacy, ${ }^{2}$ Hematology, Complejo Asistencial Universitario De Salamanca, Salamanca, Spain

Background and objective: Evaluation of the pharmacotherapeutic interventions carried out by a clinical pharmacist in a hematology unit, in order to determine the usefulness of incorporating a pharmacist into the clinical teams of these units.

Setting and method: Prospective study of the pharmaceutical interventions carried out by a pharmacist incorporated into a reference hematology unit and who tended patients undergoing allogenic bone marrow transplant for 2 months (August-September 2017).

Main outcome measures: The patient variables recorded were: sex, age, pathology that required the transplant, and length of hospital admission.

Regarding the interventions, the variables recorded were the types of intervention and the acceptance rate.

Results: A total of 192 interventions were documented in 25 patients, with a median age [range] of 56.5 [22-68], of whom $20 \%$ were women. The hematological pathologies for which the transplant was required were: acute myeloblastic leukemia (44\%), non-Hodgkin lymphoma (16\%), myelodysplastic syndrome (16\%), acute lymphoblastic leukemia B (12\%), Hodgkin lymphoma (4\%), Philadelphia + acute lymphoblastic leukemia (4\%) and acute biphenotypic leukemia (4\%). The median duration of admission [range] was 31 [24-257] days.

Pharmacotherapeutic interventions carried out were classified into the following categories: recommendation to add a medication for an untreated pathology (18.7\%), electrolyte adjustment (10.4\%), dose adjustment for an erroneous dosage $(9.4 \%)$, antibiotic treatment discontinuation due to excessive duration $(9.4 \%)$, pharmacokinetic monitoring recommendation for dose adjustment (9.4\%), nutritional intake intervention $(9.4 \%)$, stopping a prescription for an unnecessary medication and/or one that was duplicated $(8.3 \%)$, treatment conciliation at admission $(7.3 \%)$, erroneous or wrong treatment with proposal to modify the medication $(5.2 \%)$, dose adjustment in patients with reduced kidney function $(5.2 \%)$, and others $(7,3 \%)$.

The acceptance rate of pharmaceutical interventions was $83.3 \%$. Conclusion: The integration of a pharmacist into multidisciplinary teams for patient care contributes to the prevention, detection and resolution of problems related to medications. The high rate of acceptance for the pharmaceutical interventions demonstrates the advantages for improving healthcare and safety for patients that would accrue from incorporating a pharmacist on a daily basis.

Disclosure of Interest: None Declared. 
HP-PC062: Guidelines compliance of oral anticoagulants in geriatrics ordered and monitoring in a French hospital group

L. Denis ${ }^{1, *}$, M. Fodil ${ }^{2}$, R. Sterpu ${ }^{3}$, N. Ricome ${ }^{2}$, S. Drouot ${ }^{1}$, A. Rieutord ${ }^{3}$, L. Duranteau ${ }^{1}$, C. Verny ${ }^{1}$, M. C. Chaumais ${ }^{3}$

${ }^{1}$ AP-HP, Hôpital Bicêtre, Le Kremlin-Bicêtre, ${ }^{2}$ AP-HP, Hôpital Paul Brousse, Villejuif, ${ }^{3}$ AP-HP, Hôpital Antoine Béclère, Clamart, France

Background and objective: Within the geriatric population, an increasing use of oral anticoagulants (OAC), Direct Oral Anticoagulants (DOAC) and Vitamin K Antagonist (VKA), is observed. However, the respect of guidelines regarding drug monitoring for VKA, and dosage adjustment for DOAC according patient characteristics and therapeutic indication remains a challenge. In 2017, the Drug Committee of our teaching hospital addressed the compliance of anticoagulant prescription and monitoring. For DOAC, the principal objective was to check the conformity according to the guidelines as absence of contra-indications and appropriate adjustment to biometrics and biologic data. International Normalized Ratio (INR) monitoring and dose adjustment were verified for VKA use.

Design: One-day quality audit through a transversal and multi-centre study including geriatric patients aged of at least 75 years with an oral anticoagulant.

Results: 108 patients were included. Mean age was 86.2 years (SD: 84.9-87.1 years) with twice more women than men (sex ratio $=$ $0.46)$. The study population was distributed according to treatment type with a large majority for VKA: warfarin $(n=59,55 \%)$, fluindione $(\mathrm{n}=20,18 \%)$, apixaban $(\mathrm{n}=19,18 \%)$, rivaroxaban $(\mathrm{n}=7$, $6 \%)$, dabigatran $(\mathrm{n}=3,3 \%)$. Conformity rate in DOAC was $79.3 \%$. Non-conformities were 4 under-dosing and 2 overdosing. Conformity rate in VKA was $51.9 \%$ due to incorrect INR monitoring and inappropriate related dose adjustment.

Conclusion: In our hospital, most DOAC prescriptions within the geriatric patient-population met the guidelines but, it remains a challenge. In our study, VKA monitoring and related dose adjustment was insufficient and may lead to severe clinical consequences. Pharmacists have to be aware when undertaking patient medication review to optimize treatment conformity with physicians by improving anticoagulant treatment monitoring and dose-adjustment algorithm.

Disclosure of Interest: None Declared.

HP-PC063: Improving our practice of clinical pharmacy: let's work together !

\section{N. d'Houdain ${ }^{1, *}$, H. Mazaré ${ }^{1}$, D. Marconot ${ }^{1}$, C. H. Blancher ${ }^{1}$, E. Queuille ${ }^{1}$}

${ }^{1}$ Pharmacy, Charles Perrens Psychiatric Hospital (Bordeaux), Bordeaux, France

Background and objective: Polypharmacy, especially in elderly people, causes an increase of iatrogenesis, by pharmacological effect, as well as by poor compliance issues, because of the complexity of the prescription. In our hospital, analysis of prescriptions (AOP) is performed daily. Retroactive medication reconciliations are performed in ten care units and prescription review program (PRP) is set up in two care units. Through the analysis of discharge prescriptions, the goal is to draw the axes of improvement leading to the optimization of these prescriptions within our psychiatric institution.

Design: Analysis of discharge prescriptions were performed in patients older than 65 years old, receiving medication reconciliation upon admission in 2017 using the EU(7)-PIM list as reference. Demographic characteristics, number of drugs on admission and discharge, number of benzodiazepines (BZD) and antipsychotics (AP) (including BZD) were assessed. Pharmacists of the unit defined other iatrogenesis criteria, in addition to unsuitable drugs, like the number of drugs on demand, overdoses/marketing authorization (OMA), drugs suspended and modified on admission for referenced drugs, and not renewed at discharge (DNR) and finally any other drug considered inappropriate (other) and not listed on the EU(7)-PIM list.

Results: 233 patients were included in the study; ratio F/M: 2.32; mean age: $74.2 \pm 6.2$ years; mean number of drugs on admission: $7.8 \pm 3.2$; on discharge: $8.9 \pm 3.6$; mean AP: $2.6 \pm 0.84$ and mean BZD: $0.90 \pm 0.33$. The mean number of drugs on The EU(7)-PIM list was 1.2 (BZD: $39 \%$, proton pump inhibitors (PPI): $24 \%$, antidepressant: $16 \%$ ); the mean number of drugs on demand: 1.4 (BZD: $33 \%$, analgesics: $24 \%$, laxatives: $14 \%$ ); the mean number of OMA: 0.27 (laxatives: $63 \%$, analgesics: 16\%); the mean number of DNR: 0.42 (antihypertensive agents: $32 \%$, PPI: $24 \%$, statins: $11 \%$ ) and the mean number of other: 0.33 (long half-life BZD: 25\%; oral liquid forms: $18 \%$ ).

Conclusion: Therapeutic optimization is a public health issue, both for the patient and economic reasons. The use of a the EU(7)-PIM list in this process is useful although it seems incomplete in several points especially about long half-life BZD (prazepam, clorazepate) and antidepressants, commonly used in our psychiatric institution. With $72 \%$ of prescriptions containing at least $\geq 1$ drugs on demand, the risk is that patients abuse it by forgetting intake or by impression of possibility of excessive intake. Drugs which are unavailable at hospital are switched on admission but not renewed at discharge leading to a risk of resumption of the old treatment at home and therefore redundant treatments. OMA were mostly related to drugs used for corrective purposes because of side effects of AP drugs. Despite the daily AOP, the mean number of drugs at discharge increased by $12 \%$ compared to admission. However, this value tends to decrease in care units where PRP is set up. Targeted actions such as the PRP or proactive medication reconciliations before discharge will have more impact and appear to be a feasible solution in optimizing discharge prescriptions.

Disclosure of Interest: None Declared.

HP-PC065: Allelic frequencies differences of relevant pharmacogenetic polymorphisms in Europe compared to our population

X. Díaz-Villamarín ${ }^{1}$, M. Cameán-Castillo ${ }^{2}$, E. García-Lobato ${ }^{3}$, D. Blánquez-Martínez ${ }^{1}$, A. Antúnez-Rodríguez ${ }^{4}$, E. Leno-Durán ${ }^{4}$, M. Pérez-Campos ${ }^{1}$, I. Cano-Talavera ${ }^{1}$, R. Herrera-Trigueros ${ }^{1}$, L. J. Martínez-González ${ }^{4}$, C. L. Dávila-Fajardo ${ }^{1}$, R. Álvarez-Sánchez ${ }^{1, *}$

${ }^{1}$ Clinical Pharmacy, San Cecilio University Hospital, Granada, ${ }^{2}$ Clinical Pharmacy, Hospital Universitario Puerto Real, Cádiz, ${ }^{3}$ Clinical Pharmacy, Comlejo Hospitalario Universitario de Badajoz, Badajoz, ${ }^{4}$ Genomics Unit, GenYo, Granada, Spain

Background and objective: Pharmacogenetics enables different responses to drugs among patients to be identified and predicted. During these last years, pharmacogenetic dosing guidelines have been developed and its use implemented in daily clinical practice around the world. But, not all the single nucleotid polymorphisms (SNP) affecting drugs response are similarly relevant among populations if we look at their allelic frequencies, even if we compare these frequencies in Europe and one of its subpopulations.

The aim of this study is to find, among the SNP studied by the pharmacogenetics unit of our hospital, those with different allelic frequencies compared with the European population.

Setting and method: The patients' genotypes of all the SNP studied by the pharmacogenetics unit were recorded since the first pharmacogenetic test performed in 2012. 
The SNP's were genotyped using predesigned TaqMan ${ }^{\circledR}$ genotyping assays technology or KASP assay technology and analyzed on a 7900HT Fast Real-Time PCR System de Applied Biosystems.

Main outcome measures: The allelic frequencies of the studied SNP were calculated and compared using Chi square test or fisher exact test. $P$ values less than 0.05 were considered statistically significant. Results: Since the first pharmacogenetic test performed by our department, 7.678 tests in 2.287 patients, affecting 7 drugs, were performed.

Altogether, 27 different SNP were genotyped. From these, we found that 3 SNP show significant differences about their allelic frequencies compared with those reported by the 1000 Genomes Project for the European population. These are: ABCB1 C3435T (c.3435T $>$ C; rs 1045642) that showed a minor allele frequency (MAF) of $48.2 \%$ in the European population and $44.8 \%$ in our population $(p=0.046)$; FcGR *3A (c.841T > C; rs396991) $(73.1 \%$ vs $62.8 \% ; p<0.001)$ and CYP2D6*5 (gene deletion)(7\% vs $0 \% ; p=0.012$ ).

Conclusion: There are differences between relevant pharmacogenetic polymorphisms allelic frequencies comparing a reference population (Europe) and one of its subpopulations.

Disclosure of Interest: None Declared.

\section{HP-PC066: Experience of use and safety of apremilast in a tertiary level hospital}

N. Fernandez Bargiela ${ }^{1, *}$, V. Giménez Arufe ${ }^{1}$, L. Elberdin Pazos ${ }^{1}$, Á. Porta Sánchez ${ }^{1}$, C. Mondelo García ${ }^{1}$, C. Fernández Oliveira ${ }^{1}$, I. Martín Herránz ${ }^{1}$

${ }^{1}$ Hospital Pharmacy, Complexo Hospitalario Universitario A Coruña, A Coruña, Spain

Background and objective: Apremilast has emerged as a promising new oral therapy in psoriasis and psoriatic arthritis. It is an oral smallmolecule inhibitor of phosphodiesterase 4 intracellularly, modulates the expression of TNF- $\alpha$, IL-23, IL-17 and other inflammatory cytokines and levels of anti-inflammatory cytokines such as IL-10; these pro- and anti-inflammatory mediators have been implicated in inflammatory diseases. The main objective of our study is to describe the use and safety of apremilast in a tertiary hospital.

Design: Observational retrospective study of all patients treated with apremilast was carried out from January 2017 to March 2018. Data sources: electronic medical records (IANUS ${ }^{\circledR}$ ) and prescription program $\left(\right.$ Silicon $\left.^{\circledR}\right)$. Statistics: descriptive analysis of variables (Microsoft Excel) qualitative variables (median/range) and quantitative (percentage).

Variables collected: sex, age, body mass index (BMI), diagnostic, posology, previous treatment with biologist drugs, concomitant treatment with immunosuppressive drugs (methotrexate, leflunomide and azathioprine), duration of treatment, adverse events (AE) and discontinuation causes.

Results: 26 patients (54\% women). Age: 54 (19.6-78.9) years old. Median BMI: $30.1 \mathrm{~kg} / \mathrm{m}^{2}$ (23.9-46.2). Diagnostic: 7/26 (26.9\%) psoriatic arthritis (PA), 9/26 (34.6\%) moderate to severe psoriasis, 8/26 (30.8\%) Behçet's syndrome (off-label use) and 2/26 (7.7\%) PA and psoriasis. Posology: 25/26 (96.2\%) $30 \mathrm{mg}$ twice daily after initial titration schedule, $1 / 26(3.8 \%)$ reduce apremilast dose to $30 \mathrm{mg}$ once daily after initial titration adapted due to severe renal impairment. Biologic drug pre-treatment: 11/26 (42.3\%). Concomitant treatment: 5/26 (19.2\%). Median duration of treatment at April 2018: 1339 days (19-3562), median 105 days (16-2334) among patients who discontinued the treatment. AE: $38.5 \%$ gastrointestinal disorders; $19.2 \%$ headache; $15.4 \%$ psychiatric disorders, such as insomnia and depression; $7.7 \%$ body weight loss and decreased appetite; $3.8 \%$ chickenpox. Discontinuation causes [10 patients (38.5\%)]: 8/10 (80.0\%) AE, 2/10 (20.0\%) lack of efficacy.
Conclusion: Apremilast is widely used in our center, beyond the indications authorized in the technical specifications such as Behçet's syndrome. However it has a high incidence of adverse side effects, which can cause the treatment's discontinuation.

Disclosure of Interest: None Declared.

HP-PC067: Epidemiological analysis of pressure ulcers following the introduction of monitoring software

E. Galfrascoli ${ }^{1}$ on behalf of Multidisciplinary Group PUs, E. Calzavara ${ }^{1, *}$, S. Campbell Davies ${ }^{1}$, L. Gambitta ${ }^{1}$, P. Richelmi ${ }^{1}$, G. Muserra ${ }^{1}$ on behalf of Multidisciplinary Group PUs - Asst Fatebenefratelli Sacco, Po Fatebenefratelli

${ }^{1}$ Hospital Pharmacy, Asst Fatebenefratelli Saccoatebenefratelli Sacco - Po Fatebeneffratelli, Milan, Italy

Background and objective: Pressure ulcers (PUs) are often managed as a secondary problem, since the primary target is the resolution of the major disease. As their poor management may lead to a longer hospitalisation, with heavier nurses' workload, an increase in secondary costs and patients' discomfort, it is necessary to raise the awareness and improve both tools and clinical pathways, which can concretely help healthcare providers in managing patients with a high risk to develop PUs. To achieve this goal, a multidisciplinary group, coordinated by a hospital pharmacist was created in our hospital and a software to monitor PUs' treatment was developed. The aim of this work is to analyse the data collected during the first year of implementation (2017).

Setting and method: In 2017, a software was introduced, in order to help healthcare providers in monitoring the current state of PUs management. It consists of a web-based platform, where nurses are required to fill in several fields: patient's name and surname, date of birth, date of admission, hospitalisation ward, PUs' staging, anatomic sites involved accompanied by the type and number of wound dressings requested to the hospital pharmacy to treat the specific wound. Moreover, 3 internal training courses were organised to train nurses in recognising PUs and managing them using the proper medications. Data were exported in an excel sheet.

Main outcome measures: Main outcome measures extracted and analysed were:

- Number of patients and wards involved;

- Number, stage and anatomic site of PUs;

- Possible improvements in treating PUs.

Results: In 2017, 335 patients were treated: $56.42 \%(\mathrm{~N}=189)$ were females. The total number of PUs managed was 416 and only the 25.96\% ( $\mathrm{N}=108)$ were developed during hospitalisation. The majority of PUs, both already present at hospital admission $(36.06 \%$; $\mathrm{N}=150)$ and developed during hospitalisation $(14.42 \% ; \mathrm{N}=60$ ), were stage 2 . The most involved anatomic sites were sacrum $55.29 \%$ $(\mathrm{N}=230)$, heel $16.59 \%(\mathrm{~N}=69)$ and gluteus $10.34 \%(\mathrm{~N}=43)$. The wards with the higher number of PUs were pulmonology with $22.84 \%$ $(\mathrm{N}=95)$ of total injures, followed by gastroenterology $14.42 \%$ $(\mathrm{N}=60)$. The main number of sacrum PUs was detected in pulmonology $\mathrm{N}=65$, internal medicine $\mathrm{N}=40$ and gastroenterology $\mathrm{N}=31$. The main number of heel and gluteus PUs was observed in the department of intensive care $\mathrm{N}=11$. Comparing the requests of wound dressings between 2016 (period before the introduction of the software) and 2017 (after the introduction of the computer program), it was noticed a reduction in use of medications containing antimicrobials and ones approved for infected PUs. Analysing the data acquired, there seems to be a more appropriate choice of medications. Conclusion: Multidisciplinary team work, assisted by a specific training programme and dedicated software, appears to be useful to 
monitor PUs and their treatment in our hospital. These data are preliminary, and further improvements of the computer program will be carried out, in order to check appropriatness of the management (identification and treatment) according to the stage of the wound.

Disclosure of Interest: None Declared.

\section{HP-PC068: Management of outpatient parenteral antibiotic therapy in Cystic Fibrosis: a survey of hospital pharmacies in France, United Kingdom, Ireland, Belgium and The Netherlands}

\section{H. Rouzé ${ }^{1, *}$, S. Garcia ${ }^{1}$, L. Margueritte ${ }^{1}$, M. Diet ${ }^{1}$, I. Carpentier ${ }^{1}$ \\ ${ }^{1}$ Pharmacie Centrale, Hospices Civils de Lyon, Saint-Genis Laval,} France

Background and objective: Our pharmaceutical unit works with a specialist CF center and is dedicated to centralised preparation of ready-to-use antibiotic portable devices for Cystic Fibrosis (CF) outpatients. This activity is scarcely developed in France. There is also a lack of data regarding pharmaceutical involvement in $\mathrm{CF}$ management in France and other European countries ${ }^{1}$.

The aim of this work was:

- to describe French hospital pharmacies activities toward CF outpatient,

- to compare French practices with other European countries especially in the field of intravenous antibiotic centralised preparations.

Design: A survey was performed to evaluate the involvement of hospital pharmacies and submitted to:

- Pharmacies located in hospitals harboring French specialist CF centers (33),

- The members of the European Cystic Fibrosis Pharmacists group (ECFPG) through mailing and the NHS "Cystic Fibrosis Pharmacists Group" network (25).

Results: Thirty-five pharmacies answered the survey: 16 French, 16 from United Kingdom and Ireland, 2 from Belgium and 1 from The Netherlands. One-third of the French pharmacies are attached to a $\mathrm{CF}$ multidisciplinary team (MDT) and mostly provide prescription monitoring. In other countries all pharmacies report a clinical pharmacy activity within a MDT (CF MDT meeting, medicines management and adherence program, patient counselling and medication review...). Centralised preparation is not reported by other French hospital pharmacies, but $9 / 19$ of responders outside of France have such an activity, mostly in a dedicated aseptic unit; at least 6 of them for outpatients. The average number of patients per month is 28 [5-75], with an average of 619 antibiotic preparations each month. This is comparable to our own activity.

Intravenous antibiotics for centralised preparations are mostly prescribed by $\mathrm{CF}$ specialist physicians $(80 \%)$. In France and Belgium, home antibiotics are administered only by nurses. Other responders report mainly patient self-administration.

Main antibiotics prepared are Tobramycin, Ceftazidime (80\%), Piperacillin-tazobactam, Meropenem (70\%), Amikacin (60\%), Flucloxacillin and Aztreonam (50\%). Therapeutic drug monitoring is done by $7 / 9$ pharmacies for aminoglycosides and $4 / 9$ for glycopeptides.

Conclusion: This survey shows real differences in the manner French hospital pharmacists are involved in the provision of $\mathrm{CF}$ outpatient pharmacy clinical services, compared to non-french ECFPG members. We identified ECFPG members, having the same activity of centralised antibiotic preparation for CF patients. It will allow us to discuss and agree standards of practice as were described for other clinical pharmacy services.

\section{Reference}

1. Thompson K. et al. Role of the clinical pharmacist in the management of CF. Hospital Pharmacy Europe 2015;80.

Disclosure of Interest: None Declared.

HP-PC069: Medicines optimisation for ambulatory patients of a general hospital

D. Gennimata ${ }^{1, *}$, F. Marini ${ }^{1}$, I. Panderi ${ }^{2}$

${ }^{1}$ Pharmacy, "Korgialenio-Benakio" Red Cross General Hospital, ${ }^{2}$ Laboratory of Pharmaceutical Analysis, Division of Pharmaceutical Chemistry, School of Pharmacy, National and Kapodistrian University of Athens, Athens, Greece

Background and objective: According to national legislation, certain groups of ambulatory patients are eligible to, free of charge, dispensing of their medication, in public hospital pharmacies. Therefore, medicines optimisation is essential for cost-effective and safe treatment of diseases and hospital pharmacists are engaged in safeguarding this, providing clinical services in addition to their administrative ones.

Setting and method: Outpatients receiving their medication (other than for HIV treatment, previously described) from our hospital pharmacy, during the period of 2011-2017, participated in this study. Access to medication (shortages and/or overprescription), duration and cost of treatment, drug interactions and adverse effects reported during medication review, were registered in the Hospital Information System and were analyzed by Excel ${ }^{\circledR}$ and SPSS ${ }^{\circledR}$.

Main outcome measures: An evaluation of medicines optimisation services provided to our hospital outpatients (not including the HIV ambulatory patients) is presented in this study.

Results: During 2011-2017, medication was dispensed to 3458 outpatients (not including the HIV ambulatory patients), in an average of 944 per year (2011-2016) and 149 in 2017, due to changes in legislation. Shortages appeared to an average of $10 \%$ and were managed in collaboration with the prescribing clinician. Overprescription was averted in $25 \%$ of all prescriptions, through medication review. Cardiovascular and nervous system agents were among the most prescribed (20\% and $40 \%$ respectively), whereas alimentary tract and metabolism drugs and antimicrobials constituted an average of $15 \%$ and $11 \%$ respectively. An average of $95 \%$ of those outpatients was treated for chronic conditions and the average cost per patient was $720 €(2011-2016)$ and $1500 €(2017)$, representing 9\% of the annual hospital budget for pharmaceuticals in 2011-2016 and 2\% in 2017. Drug interactions and adverse effects were prevented in $5 \%$ of all prescriptions, by the medication review at the pharmacy.

Conclusion: Medicines optimisation services that are designed to improve patient safety and manage public spending are included in the dispensing of medication to outpatients and are provided by the hospital pharmacists. Medication review of outpatient prescriptions facilitated prevention of adverse drug events and cost-effective access to pharmaceutical treatment of diseases. Further analysis of data is currently under way to evaluate more aspects of medicines optimisation (e.g. reconciliation, polypharmacy, etc.)

Disclosure of Interest: None Declared.

HP-PC071: Medication discrepancies between a standard medication history and a best possible medication history performed by a clinical pharmacist

S. S. Gut ${ }^{1,2, *}$, P. Imfeld ${ }^{1}$, A. B. Leuppi-Taegtmeyer ${ }^{1}$, D. Bornand ${ }^{1}$, S. Bassetti ${ }^{1}$, C. R. Meier ${ }^{1,2}$

${ }^{1}$ University Hospital Basel, ${ }^{2}$ University of Basel, Basel, Switzerland 
Background and objective: Improper documentation of patient's drug therapy at hospital admission often leads to drug related problems during hospitalisation or after discharge. There's little data on the quality of medication histories of newly admitted patients to Swiss hospitals. This study aimed to investigate the number and type of medication discrepancies between a standard medication history $(\mathrm{SMH})$ and a best possible medication history (BPMH).

Setting and method: At the University Hospital Basel, assistant physicians perform the SMHs on admission at the emergency department. During a 2-month period, a clinical pharmacist performed a BPMH on an internal medicine ward in addition to the $\mathrm{SMH}$. The study population consisted of patients, transferred from the emergency department to the internal medicine ward. We performed the BPMH $24 \mathrm{~h}$ after the patient's transfer, from Monday to Friday between 12:00 and 14:00. This time slot was the limiting factor for the number of BPMHs possible to perform per day. To compile the BPMH we used at least two independent information sources, whereof one was a patient interview if feasible. For each patient we compared the SMH with the BPMH and assessed the number and type of medication discrepancies. We recorded the time to perform a BPMH.

Main outcome measures: Number and type of medication discrepancies. Time required to perform a BPMH.

Results: We performed a BPMH in 55 patients $(22$ females, mean age 66 years) with a total number of 465 drugs. The average number of drugs per patient was 8.5 . We identified 227 discrepancies between the BPMH and the SMH, resulting in 4.1 discrepancies per patient on average. Most common types of medication discrepancies: drugs not documented in the SMH but taken by the patient $(\mathrm{n}=125,55 \%)$, discrepant dosages $(n=64,28 \%)$, documented drugs in the SMH which the patient didn't take $(\mathrm{n}=30,13 \%)$.

We needed on average $35.5 \mathrm{~min}$ to perform a BPMH.

Conclusion: This study revealed that SMHs are often incomplete. The performance of a standardized BPMH by a clinical pharmacist seems to be a promising approach to generate an accurate drug list at admission to hospital. The fact that performing a BPMH is very timeconsuming might be a limitation for a broad implementation.

Disclosure of Interest: None Declared.

HP-PC072: Administration of levetiracetam in subcutaneous infusion: a case report

A. Heim ${ }^{1, *}$, M. Mehring ${ }^{1}$, A. Bouothmani ${ }^{1}$, A. Pagès ${ }^{1}$, C. Peyrol ${ }^{1}$, C. McCambridge ${ }^{1}$, P. Cestac ${ }^{1}$, A.-S. Mangé ${ }^{1}$

${ }^{1}$ CHU Purpan, Toulouse, France

Background and objective: The subcutaneous route is often characterised by its effectiveness, safety and comfort for the patient. This administration technique needs to be better explored because data from literature remains limited.

The aim of this work is to describe the case of a patient who received levetiracetam in subcutaneous infusion and to evaluate its short-term effects.

Design: Case report.

Results: An 85-year-old woman living at home and recently diagnosed with a cognitive impairment, was found unconscious.

On admission to the emergency room, the patient was still in a comatose state, suffering from hypothermia but having good hemodynamic functions. Lower limb myoclonus was also reported.

Seven days later, she had a generalized tonic-clonic seizure for less than $1 \mathrm{~min}$, which resolved spontaneously.

The Computed Tomography brain scan did not show any suspicious lesion and was similar to the previous one. An electroencephalogram (EEG) showed evidence of metabolic encephalopathy, but no epilepsy. The patient had recently received ceftriaxone infusions complicated by acute renal failure in a context of urinary tract infection.

During her stay in hospital, the patient was alternatingly in a wakeful then drowsy state. She had two other tonic-clonic seizures 10 and 16 days after the first one. After the second seizure, IV administration of levetiracetam was chosen because of the risk of a worsening disturbed vigilance caused by benzodiazepines.

Facing the difficulty to keep IV route because of patient's agitation and the impossibility to use oral path, the subcutaneous route was suggested as an alternative.

In the described conditions of administration, the patient received $250 \mathrm{mg}$ of levetiracetam twice a day in the form of a $30 \mathrm{~min}$ infusion (dilution with $100 \mathrm{~mL}$ of sodium chloride $0.9 \%$ ). In total, 32 levetiracetam subcutaneous infusions occurred without any significant adverse effects.

Then, the treatment was switched to oral levetiracetam, $250 \mathrm{mg}$ every $12 \mathrm{~h}$. The clinical state of the patient improved and she recovered a normal state of awareness. No other EEG was performed. Conclusion: In these conditions, this case indicated that the subcutaneous route seems efficient, well tolerated and could be considered as an alternative to the IV administration of levetiracetam.

Disclosure of Interest: None Declared.

HP-PC073: Patients with coronary heart disease who have negative beliefs about their medicines report lower adherence

\section{Hellström ${ }^{1, *}$, M. J. Östbring ${ }^{2}$}

${ }^{1}$ Pharmaceutical Department, Kalmar County Council, ${ }^{2}$ eHealth Institute, Dep of Medicine and Optometry, Linnaeus University, Kalmar, Sweden

Background and objective: An intervention was developed to influence patients' beliefs about medicines with the intention to increase patients' adherence. This intervention was tested in a randomized controlled trial (RCT) in patients with secondary prevention for coronary heart disease (CHD). The randomization was stratified based on beliefs and the intervention was intensified for patients with negative beliefs. The objective of this study was to explore differences at baseline between patients with accepting attitude, and those holding negative beliefs, i e ambivalent, neutral or skeptical attitude towards their heart medicines. Setting and method: Observational study. Data collected from an RCT conducted at the cardiology clinic of a teaching hospital in Sweden. Sociodemographic and medical data collected in connection to discharge. Questionnaires for adherence to cholesterol lowering medicines and beliefs about heart medicines were collected 3 months after discharge. $\mathrm{Chi}^{2}$ test was used to compare groups.

Main outcome measures: We used attitudinal analysis of the Beliefs about Medicines Questionnaire (BMQ)-Specific. The attitudinal categories were compared in terms of self-reported adherence dichotomized to high or low.

Results: Data on self-reported adherence and BMQ-Specific were available from 309 patients. Mean age was $68.5(8.9)$ y and $229(74 \%)$ were men. $153(49.5 \%)$ patients had an accepting attitude, $99(32 \%)$ had an ambivalent attitude, $34(11 \%)$ were neutral and $23(7.4 \%)$ patients had a skeptical attitude. Accepting patients did not differ from patients with negative attitude in terms of education level $(p=0.53)$, living alone $(p=0.21)$, newly onset of CHD $(p=0.54)$ or if they suffered from acute CHD or not $(p=0.63)$.

Among accepting patients $8(5 \%)$ reported low adherence to cholesterol lowering medicines, while $20(14 \%)$ of patients with negative beliefs reported low adherence $(p=0.014)$.

Conclusion: Self-reported low adherence to cholesterol lowering medicines was more common among patients with negative beliefs about medicines enrolled in this RCT.

Disclosure of Interest: None Declared. 
HP-PC074: Multidose drug dispensing; aid or obstacle for adherence to phosphate binder? A case study

\section{B. J. Hjemås ${ }^{1, *}$, K. Bøvre ${ }^{1}$, L. Mathiesen ${ }^{2}$, K. Bjerknes ${ }^{1}$}

${ }^{1}$ Hospital Pharmacies Enterprise, South Eastern Norway, ${ }^{2}$ Department of Pharmaceutical Biosciences, School of Pharmacy, University of Oslo, Oslo, Norway

Background and objective: A pharmacist-led intervention including education and counselling was applied in a study on how to improve adherence to phosphate binder in dialysis patients. Some of the patients in the study used multidose drug dispensing (MDD). In conversation with these patients, various challenges associated with the use of multidose were revealed.

Design: We share a case report on a participant using MDD in the study performed on dialysis patients at a hospital in Norway. To measure self-reported adherence, beliefs about medicines and knowledge, the MARS-5 questionnaire, the BMQ questionnaire and a knowledge questionnaire was used respectively.

Results: One multidose user reported that he previously had taken phosphate binder, but that this no longer was part of his medication regime. In the medication interview, it was revealed that tablets that the patient believed were painkillers were identified to be phosphate binders. Since the patient did not experience pain, he had chosen not to take these tablets. The patient score on the MARS-5 questionnaire was high before (21) and after (23) intervention in spite poor adherence regarding phosphate binder prior to the intervention. He was unsecure regarding phosphate binder necessity and worries, seen in the BMQ both prior to and after intervention. The patient answered correctly on three of eight questions regarding phosphate binders prior to and five after the intervention. No difference was seen in serum phosphate during the study. After counselling by the pharmacist, the patient started to take his phosphate binder.

Conclusion: Inability to identify individual tablets in MDD may increase the risk of non-meal intake or no intake at all if phosphate binders are mistaken to be other medications the patient has made decisions not to take. Individual counselling of the patient regarding medication reveals these misunderstandings.

Disclosure of Interest: None Declared.

\section{HP-PC075: Experience of clinical pharmacy services} in an intensive care unit

\section{K. Ioannidis ${ }^{1, *}$, A. Papachristos ${ }^{1}$, I. Scarlatinis ${ }^{1}$ \\ ${ }^{1}$ Clinical Pharmacy, Hygeia Hospital, Athens, Greece}

Background and objective: Intensive Care Unit (ICU) is a setting where adverse drug events often occur. Thus, critical care pharmacists participate in routine medical rounds they perform therapeutic drug monitoring, daily medication review, and drug history evaluation and generally they are responsible for pharmacotherapy modification and correct use of medication. Although the role of clinical pharmacist in ICU is well-established in many regions, Greek hospitals have still not taken advantage of those services.

Design: An observational study was performed by analyzing interventions of clinical pharmacist during a period of 6 yeras (2010-2016) in ICU with 35 beds, where adult patients are hospitalized. All intervations were clinically significant, accepted by medical staff and recocorded to a specific database.

Results: 12.1 intervations per 1000 patients days were recorded. The most frequent intervention was the addition of a drug (22.2\%), followed by correction of high $(17.1 \%)$ and low (16.6\%) doses. Other common intervetions include drug interactions (13.9\%), unnecessary drug withdrawals $(7.6 \%)$, administrative procedure $(4.8 \%)$, drug replacements $(4.6 \%)$, transcriptions errors $(3.5 \%)$, dilution $(4.1 \%)$, adverse events resulting in drug cascades (2.9\%) and monitoring activities (2.7\%).

Conclusion: In conclusion, this study is the first at a Greek hospital and highlights the contribution of clinical pharmacist to the optimization of pharmacotherapy in critically ill patients. Finally, antiinfectives and anticoagulants are the two crucial drug classes that critical care to which the clinical pharmacist should pay attention. Disclosure of Interest: None Declared.

HP-PC076: Clinical pharmacist-led medication review to evaluate inappropriate prescriptions in pediatric patients

M. N. Isik ${ }^{1, *}$, N. Dalgic ${ }^{2}$, B. Okuyan ${ }^{1}$, Z. Y. Yildirmak ${ }^{2}$, G. Kose ${ }^{2}$, N. Urganci ${ }^{2}$, A. Kaya ${ }^{2}$, N. Akinci ${ }^{2}$, M. Sancar ${ }^{1}$

${ }^{1}$ Clinical Pharmacy, Marmara University, ${ }^{2}$ Saglik Bilimleri

University Sisli Hamidiye Etfal Education and Research Hospital, Istanbul, Turkey

Background and objective: The aim of the present study is to identify the importance of clinical pharmacist-led medication review on inappropriate prescriptions in pediatric patients by using POPI (Pediatrics: Omission of Prescriptions and Inappropriate Prescriptions) criteria which is designed in France to determine omissons of prescriptions and inappropriate prescriptions.

Setting and method: The study was conducted between 13 November 2017-13 April 2018 in an education and research hospital in Istanbul. Prescriptions of children (aged 0-18 years) at nephrology, neurology, immunulogy and allergy, gastroenterology outpatients clinics and emergency service were evaluated.

Main outcome measures: The clinical pharmacist-led medication review performed according to the POPI propositions. POPI contained 102 propositions: 80 propositions of Inappropriate Prescription (IP) and 22 propositions of Omission of Prescription (OP) (1). However, 99 of 102 POPI propositions were included in the study, since two propositions do not comply with Turkish socio-cultural structure and one particularly drug, which is listed in POPI, does not exist in Turkey.

Results: Among 350 patients, two hundred eighty patients (142 male, 138 female) were eligible for clinical pharmacist-led medication review in pediatric patients. The median age of patients was calculated as 60 (36-108) months. According to the POPI propositions, 136 inappropriate prescriptions in 106 patients and 19 omission of prescriptions in 18 patients were determined. Prescription of oral and nasal decongestants (oxymetazoline, pseudoephedrine, phenylephrine) in treatment of ear nose and throat (ENT) infections, prescription of a drug other than paracetamol or two antipyretic at the same time in the first line treatment of pain and fever, choice of antibiotics other than amoxicillin as first line therapy in ENT infections and prescription of ketotifen and other $\mathrm{H} 1$ antagonists in the treatment of asthma are the most common inappropriate prescribing. The most common omission of prescription is not to prescribe oral rehydration solution in the treatment of vomiting and diarrhea.

Conclusion: In conclusion, the clinical pharmacist-led medication review could have beneficial effect on determination of inappropriate prescriptions in pediatric patients by using POPI. This was the first study, where the concordance of POPI criteria to determine omission of prescriptions and inappropriate prescriptions in Turkish pediatric patients, was examined. Further studies should be designed to evaluate some propositions of the POPI criteria internationally.

Disclosure of Interest: None Declared.

HP-PC077: Investigation of variables associated with medication non-adherence in patients with hypertension

A. Jarab ${ }^{1, *}$, E. A. Alefishat ${ }^{2}$, R. Bani Nasur ${ }^{3}$, T. L. Mukattash ${ }^{1}$ 
${ }^{1}$ Clinical Pharmacy, Jordan University of Science and Technology, Irbid, ${ }^{2}$ Biopharmaceutics and Clinical Pharmacy, University of Jordan, ${ }^{3}$ Pharmacy, Jordanian Royal Medical Services, Amman, Jordan

Background and objective: Despite the availability of effective treatments, outcomes are suboptimal in patients with hypertension. Medication non-adherence represents a major barrier to achieve optimal health outcomes in patients with hypertension. The study aim was to identify obstacles to medication adherence in patients with hypertension in Jordan.

Setting and method: Socio-demographic and clinical variables in addition to medication adherence and health-related quality of life were assessed for patients attending three major hospitals in Jordan. Multiple logistic regression was carried out to identify predictors of medication non-adherence.

Main outcome measures: Predictors of medication non-adherence in patients with hypertension.

Results: Of the 300 participants, $81 \%(n=57)$ were classified as nonadherent. The patients were found eight and six times less likely to adhere to their medications if they reported poor HRQol $(\mathrm{OR}=0.127$, $\mathrm{CI}=0.04-0.87)$ and if they had concerns about side effects $(\mathrm{OR}=$ $0.165, \mathrm{CI}=0.09-0.95)$ respectively. Both, the increase in the number of prescribed medications $\mathrm{OR}=0.349, \mathrm{CI}=0.17-1.19$ ) and having co-morbid illness $(\mathrm{OR}=0.315, \mathrm{CI}=0.15-1.02)$ increased the risk of medication non-adherence by three times.

Conclusion: The elements identified in the adherence model in this study including prescribing less medications, improving patients' health-related quality of life, improving perceptions about medications' side effects, and particularly addressing patients who have comorbid diseases, should guide the development of future pharmaceutical care intervention programs aimed at improving health outcomes in patients with hypertension.

Disclosure of Interest: None Declared.

\section{HP-PC078: Removal of non-current prescriptions on the shared} medication record

\section{S. Jørgensen ${ }^{1, *}$ \\ ${ }^{1}$ Capital region of Denmark, The Hospital Pharmacy, Copenhagen, Denmark}

Background and objective: The Shared Medication Record gives healthcare professionals in Denmark an overview of the patient's current medication, and it is used in all sectors to dispense and administrate from. When prescriptions are no longer current, the treating physician is obligated to remove them from the Shared Medication Record. Pharmacist have prior to implementation of a new it-platform removed the non-current prescriptions from elective patients Shared Medication Record when hospitalized. Since the ITplatform change, removal of non-current prescriptions can only be done at admission by a physician, and due to lack of time, is often neglected. This leads to patients being treated with non-current medicine when hospitalized. The aim of this study is to see if the surgeon removes non-current prescriptions upon admission when a pharmacist have listed them in a journal note.

Setting and method: Data was collected from December 18, 2017 to December 31, 2017 (2 weeks). Medical history interviews were conducted by phone prior to admission and recorded in the patient journal by a pharmacist, on elective patients from departments Center for Rheumatology and spine diseases and Orthopedic surgery at Rigshospitalet, Copenhagen, Denmark. All patients included in the study had non-current prescriptions on The Shared Medication Record and it was then checked upon admission to see if the non- current prescriptions were removed from the Shared Medication Record by the surgeon.

Main outcome measures: Number of non-current prescriptions removed from the Shared Medication Record upon admission.

Results: 23 admitted patients had 42 non-current prescriptions, where $34(81 \%)$ were removed by the surgeon on admission. 8 (19\%) prescriptions were not removed. Reasons for not removing non-current prescriptions were prescriptions to be taken by patients as needed and a new surgeon whom did not read the pharmacist note.

Conclusion: The study showed that the surgeons used the journal note made by the pharmacist, and removed the non-current prescriptions from the Shared Medication Record. By listing all noncurrent prescriptions in the journal note, the surgeons removed them from the Shared Medication Record so they no longer figure as current medication at either the hospital or after discharge.

Disclosure of Interest: None Declared.

\section{HP-PC080: A prospective study on evaluation of clinical} pharmacist's interventions regarding drug-related problems in intensive care unit

\section{E. Karatas Kocberber ${ }^{1, *}$, S. Apikoglu-Rabus ${ }^{2}$}

${ }^{1}$ Clinical Pharmacy Department, Faculty of Pharmacy, Istanbul University, ${ }^{2}$ Clinical Pharmacy Department, Faculty of Pharmacy, Marmara University, Istanbul, Turkey

Background and objective: Intensive care unit is one of the clinics where drug-related problems are often seen due to polypharmacy and critical conditions of the diseases. Intensive care pharmacy practice has become an essential component of the multidisciplinary practice since the early 1970s and critical care pharmacy residency program began in the USA in the 1980s. The pharmacist in the intensive care unit should provide medication related information to the health-care team on issues including indications, doses, drug interactions, current guidelines, drug allergies, pharmacokinetic calculations, pharmacogenetic differences, therapeutic drug monitoring and drug-induced problems. The purpose of this present study is to evaluate the clinical pharmacist's interventions and their outcomes regarding drug-related problems in the intensive care unit.

Setting and method: This prospective study was conducted on 100 patients who were hospitalized at the intensive care unit of a university hospital. Drug-related problems identified by the clinical pharmacist and proposed interventions were classified using PCNE classification scheme for drug-related problems.

Main outcome measures: Main outcome measures were the descriptive properties of the drug-related problems; and the rate of acceptance and outcomes of proposed interventions.

Results: The clinical pharmacist identified 144 drug-related problems. These problems were about treatment effectiveness $(51.4 \%)$, adverse reactions $(40.1 \%)$, treatment costs $(6.3 \%)$ and other issues $(2.1 \%)$. The causes of the problems were about drug selection $(66 \%)$, drug form choice $(0.6 \%)$, dose selection $(25.7 \%)$, drug use process $(2.8 \%)$ and other factors $(4.9 \%)$. The clinical pharmacist proposed interventions for all the identified drug-related problems and 134 (93.05\%) of these proposals were accepted. As a result of the pharmacist's interventions $57.6 \%$ of the problems were totally solved, $34.7 \%$ were partially solved and $3.5 \%$ were not solved.

Conclusion: In the intensive care unit, the critical care pharmacist may contribute to the treatment by identifying and helping solve the problems especially about treatment effectivenessand adverse reactions. Employment of a clinical pharmacist at theintensive care unit would result in better care of the patients through prevention and resolution of drug-related problems.

Disclosure of Interest: None Declared. 
HP-PC081: Comprehensive medication reviews in Swedish hospitals: what does the patient have to say?

\section{T. G. H. Kempen ${ }^{1,2, *}$, A. Kälvemark ${ }^{1}$, U. Gillespie ${ }^{1}$, D. Stewart ${ }^{3}$ \\ ${ }^{1}$ Uppsala University Hospital, ${ }^{2}$ Department of Medical Sciences,} Uppsala University, Uppsala, Sweden, ${ }^{3}$ School of Pharmacy and Life Sciences, Robert Gordon University, Aberdeen, United Kingdom

Background and objective: Medication Reviews Bridging Healthcare (MedBridge) is a clinical trial studying the impact of comprehensive medication reviews (CMRs), including follow-up telephone calls after hospital discharge, by ward-based pharmacists on older patients' health-related outcomes. Trials with complex interventions are often criticised because of a lack of understanding of the context, implementation and mechanism of action. Therefore, process evaluations of such trials are highly recommended to provide detailed understanding of patients' experiences. We proposed this nested-qualitative study to explore patients' experiences with and views on the CMRs and follow-up calls within the MedBridge trial. Setting and method: A qualitative design with in-depth semi-structured interviews. A purposive variation sampling approach was employed in which fifteen patients (66-94 years) were recruited from eight wards at four hospitals in Sweden, all of whom participated in the MedBridge trial. Interviews were audio-recorded, transcribed verbatim, and thematically analysed using a framework approach.

Main outcome measures: Key identified themes regarding the patients' experiences with and their views on the CMRs and followup calls.

Results: In general, patients had positive experiences with and views on the CMRs and follow-up calls. Seven key themes were identified: (1) there is a preunderstanding about the need for CMRs, but the effects on health outcomes are unknown; (2) patients have positive views on the added value of ward-based pharmacists, but (3) they have little understanding of the CMR process and the pharmacist's role; (4) patients trust healthcare professionals to make good choices; (5) they emphasize the importance of being informed about their medication, but recalling information is problematic. Other themes were: (6) time, location and other factors influencing the effects of CMRs; 7) problems with generic substitution.

Conclusion: Although patients generally have positive experiences with and views on CMRs and follow-up phone calls, some factors may negatively impact the effects of these interventions. These should be addressed in future initiatives on CMRs by ward-based pharmacists.

Disclosure of Interest: None Declared.

\section{HP-PC082: Evaluation of professional practices (EPP)} on artificial nutrition in a French hospital

\section{Lakhmi ${ }^{1, *}$, A. Roger ${ }^{1}$, P. Le Joubioux ${ }^{1}$, L. Réal ${ }^{1}$ \\ ${ }^{1} \mathrm{CH}$ Cambrai, Cambrai, France}

Background and objective: In 2012, we attended an EPP to assess the use of parenteral $(\mathrm{PN})$ and enteral nutrition (EN). This analysis highlighted an inappropriate use of PN to the detriment of EN. Improvement actions were then put in place to reverse this trend. The appropriate use of artificial nutrition is actually one of the reglementary items that the institutions ought to respect for the improvement and efficiency of cares. In this context, we decided to make a second EPP to draw up an inventory of undernutrition's management and appreciate the evolution of practices since 2012. Design: A data collection sheet was made to assess 3 dimensions: Assessment of the patient's nutritional state, Indication of the artificial nutrition, Description of the nutrition in place. The study is observational. The collection sheet is filled in for each patient from their computerized file. The results are compared to those obtained in 2012. We collected the consumptions and budget dedicated to PN and EN products in 2012 and 2017 from our management software. (This software was set up in July 2012, the consumptions of the last 6 months of 2012 were extrapolated for the whole year.)

Results: Sixty-two patients were included over 6 weeks in 2018 (93 over 8 weeks in 2012). Every patient was weighed at the entrance with a revaluation in $43 \%$ of cases $(78.5 \%$ of weighing at the entrance and $37.6 \%$ of revaluation in 2012). Albumin and prealbumin were respectively measured in $74 \%$ and $71 \%$ of cases $(87 \%$ and $45 \%$ in 2012). In both studies, the main 3 indications are identical: swallowing disorders, nutrition supplementation, resting of the digestive tract. We observed $61 \%$ of PN prescriptions and $39 \%$ of EN prescriptions (72\% of PN and $28 \%$ of EN in 2012). Several noncompliances were noted:-Concerning the route of administration: $61 \%$ of patients with $\mathrm{PN}$ may have received $\mathrm{EN}$, according to the elements found in their file. (They were 47\% in 2012.)—Concerning patients with PN without any oral intake: Only $43 \%$ of them were supplemented with vitamins and trace elements (37\% in 2012.). We consumed 7110 PN bags in 2012 and 3768 in 2017, or a decrease of $47 \%$. Concerning EN, 6682 bags were used in 2012 and 4658 in 2017, or a decrease of $30 \%$. PN cost $162,724 €$ in 2012 and $65,493 €$ in 2017, EN cost $8201 €$ in 2012 and $4734 €$ in 2017 (knowing that PN bag's cost is 15 times higher than EN bag's cost).

Conclusion: We noticed a global improvement of practices since 2012. However the rate of noncompliance is still too important and we'll have to investigate about the surprising decrease of global consumptions. These results were presented during the medical commission of the establishment. The prescribers were reminded about: PN indications, EN advantages, economic isuues and the interest of vitamins and trace elements supplementation for patient with PN but without oral intake. These elements were also detailed in our "Little Journal", edited by the pharmacy and intended for care wards. We'd like to create a mobile and multidisciplinary team, aiming at significatively improving the management of patients' nutrition.

Disclosure of Interest: None Declared.

HP-PC083: The impact of pharmacist independent prescribers in the Emergency Department of the Ulster Hospital

\section{A. Lawther ${ }^{1, *}$, E. Brownrigg ${ }^{1}$, E. Adair ${ }^{1}$, B. Carey ${ }^{1}$ \\ ${ }^{1}$ Phamacy, South Eastern Trust, Belfast, United Kingdom}

Background and objective: Pharmacist Independent Prescribers (PIP) at the Pharmacy Department, Ulster Hospital used Health and Social Care funding to pump prime a project where PIPs attended the medical post take ward round (PTWR) in the Emergency Department. Design: At each ward round the impact of PIP interventions was measured in terms of number, type and significance using the Eadon scale (1). Potential cost avoidance was measured using ScHARR (2). The patients seen on the PTWR were also triaged by the PIP according to pharmaceutical need and the numbers assigned to each category (red, amber, green) were recorded.

Results: Over 50 PTWRs were attended and 393 patients reviewed. The pharmacist was able to intervene 354 times and recommend further intreventions for medical staff. Of all interventions adding medicines to the kardex was the most frequent. The majority of PIP interventions were Grade 4 on the Eadon scale with the intervention being significant and resulting in an improvement in the standard of patient care. Cost avoidance from all interventions ranged from $£ 33,980$ to $£ 73,200$. This meant there was a $£ 3-£ 7$ return per $£ 1$ invested. 390 patients were triaged the majority into the red and amber category. 
Conclusion: PIP participation in the PTWR was hugely beneficial from a harm and cost avoidance perspective. Participation in the PTWR allowed the pharmacist to quickly categorise the patient for further pharmaceutical care. Feedback from medical and nursing staff confirmed the value of PIP participation. Further work is being completed on pharmaceutical triaging of patients in the South Eastern Health and Social Care Trust.

Disclosure of Interest: None Declared.

\section{HP-PC084: Evaluation of Clinical Pharmacist initiative to ward 14 Lagan Valley Hospital}

\author{
A. Lawther ${ }^{1, *}$, E. Moore ${ }^{2}$, C. Begley ${ }^{2}$ \\ ${ }^{1}$ Phamacy, South Eastern Trust, Belfast, ${ }^{2}$ Pharmacy, South Eastern \\ Trust, Lisburn, United Kingdom
}

Background and objective: Ward 14 Lagan Valley Hospital is a 24 bedded elderly care unit caring for both stroke patients and patients requiring rehabilitation usually post hip replacement. This ward currently has no full time clinical pharmacist and this study aimed to evaluate the impact a clinical pharmacist had on the ward by recording interventions made during the study week.

Design: Over 1 week in November 2017 a clinical pharmacist went to ward 14 to provide a clinical pharmacy service. The services included medicines reconciliation, kardex checks, checking discharge prescriptions and patient and carer counselling. Interventions were graded by pharmacy staff using the Eadon scale (1) and costed for potential cost avoidance using the ScHARR scale (2).

Results: The total number of patients with medicines reconciliation carried out was 13. The total number of patients counselled was 5. 66 prescribing interventions were made of which 54 were grade 4 or above using the Eadon scale. It was estimated using ScHARR that $£ 17,214-£ 36,124$ was saved in potential cost avoidance. The time to clinical check a script was recorded on the ward and compared to the usual pharmacy check in the dispensary. The clinical check on the ward was on average 22 min quicker than in the dispensary and the script turned around more quickly in the dispensary when a clinical check by a pharmacist was carried out on the ward.

Conclusion: The pharmacist clearly demonstrated a benefit when on the ward by means of prescribing interventions, patient counselling and crucially time savings to both the clinical check, number of medicines needed to be dispensed on each prescription and turnaround time. The pharmacist was well received by medical and nursing colleagues and a bid has been developed in an attempt to secure a permanent clinical pharmacist for this ward.

Disclosure of Interest: None Declared.

\section{HP-PC085: Medication adherence in chronic illness: do beliefs about medications play a role?}

\section{J. Lemay ${ }^{1, *}$, M. Waheedi ${ }^{2}$, S. Alsharqawi ${ }^{1}$, T. Bayoud ${ }^{2}$}

${ }^{1}$ Faculty of Pharmacy; Department of Pharmacology and Therapeutics, ${ }^{2}$ Faculty of Pharmacy; Department of Pharmacy Practice, Kuwait University, Kuwait, Kuwait

Background and objective: Several medicines are prescribed for chronic disease management, however, adherence to long-term therapy remains poor. Culture influences the beliefs about medications and ultimately, adherence to treatment. There is a paucity of data regarding beliefs about medications in the Middle East region and it remains to be determined how these beliefs would impact treatment adherence. The objective of this study is to investigate the relationship between patients' beliefs about medications with self-reported adherence to treatment among a chronically ill multi-cultural patient population.

Setting and method: A prospective cross-sectional study among patients treated for chronic illnesses in the Ministry of Health primary care clinics in Kuwait.

Main outcome measures: The main outcome measures are self-reported adherence to medications, beliefs and perceived sensitivity towards medications.

Results: Of the 783 patients, $56.7 \%$ were male, $73.7 \%$ were married, $53.3 \%$ were non-Kuwaitis and $49.4 \%$ had low income $(<1000 \mathrm{KD} /$ 3350USD monthly). Patients self-reported having a cardiovascular illness $(80.2 \%)$, diabetes mellitus $(67.7 \%)$, respiratory disease $(24.3 \%)$ or mood disorder $(28.6 \%)$. Participants had a mean of 2 comorbid illnesses and indicated taking an average of 4 prescription medicines to treat them. A structural equation model analysis showed adherence to medications was negatively impacted by higher negative beliefs towards medications (beta $=-0.46$ ). Factors associated with negative beliefs towards medications included marital status (being unmarried; beta $=-0.14$ ), nationality (being Kuwaiti; beta $=0.15$ ), having lower education level (beta $=-0.14$ ) and higher illness severity $($ beta $=0.15)$. Younger age $($ beta $=0.10)$ and higher illness severity (beta $=-0.9$ ) were independently associated with lower medication adherence. Income and gender did not influence medication adherence or beliefs about medications. The combined effect of variables tested in the model explained $24 \%$ of the variance in medication adherence.

Conclusion: Medication adherence is a complex, multifaceted issue and patient beliefs about medications contribute significantly, albeit partially, to adherence among a multi-cultural Middle Eastern patient population.

Disclosure of Interest: None Declared.

HP-PC086: Pilot study: first steps for the development of a predictive tool for delirium

\section{A. E. Lisibach ${ }^{1, *}$, P. Wiedemeier ${ }^{1}$, M. Lutters ${ }^{1}$, C. Csajka ${ }^{2}$ \\ ${ }^{1}$ Clinical Pharmacy, Hospital, Baden, ${ }^{2}$ Clinical Pharmacy, University of Geneva, Geneva, Switzerland}

Background and objective: Delirium is an acute confusional state that is rarely caused by a single factor. It is a potentially preventable source of mortality and morbidity in hospitalized elderly patients where it occurs in $20-60 \%$.

Design: This pilot study aims to collect knowledge how data are provided in patient's files and to check the plausibility of a future retrospective analysis to develop a predictive tool that will be implemented in the electronic patients record and detect automatically patients at risk for delirium. Setting and method: A retrospective study on a geriatric ward of a 360 bed cantonal hospital. $95 \%$ confidence intervals and $p$ values $(<0.05)$ were calculated using fisherexact test and $t$ test.

Results: The outcome was delirium stated in the diagnosis list or free text and compared to the delirium observation scale (DOS) measured three times a day. Risk factors: ePA-AC score, CRP, electrolytes, ECPA, homocysteine, vitamin B12, glomerular filtration rate, visual and hearing aids, alcohol abuse, parkinson, epilepsy, infection, catheter, heart disease, diabetes, kidney dysfunction, dementia, falls, stroke, depression, anticholinergic drugs. Results: 21 out of 59 screened patients met our inclusion criteria. 5 patients developed a delirium during their hospitalizations (23.8\%). Diagnosis was not always clearly stated in the diagnosis list, in 2 patients it was only found in free text. $51.1 \%$ of all DOS measures in delirious patients were $\geq 3$ compared to only $10.6 \%$ in non delirious patients. One had even scores of up to 8 . In the delirious patients the average DOS score was 3.2 compared to nondelirious patients $0.8(p<0.05)$. Most risk 
factors could be collected from patient records. Homocystein levels and the ECPA score for pain measurements were always missing. Only falls were associated with a higher risk of delirium $(p<0.05)$. Additionally, patients with delirium had a lower self-caring index and a higher CRP compared to non-delirious patients $(p<0.05)$.

Conclusion: Data were well provided. The outcome delirium was difficult to identify as also a DOS $\geq 3$ was seen in non-delirious patients. Before including other risk factors into the model, interaction in between them needs to be analysed as well.

Disclosure of Interest: None Declared.

HP-PC087: Impact of the clinical pharmacy team in fracture and falls prevention in intermediate care

\section{K. Miller ${ }^{1, *}$, C. Trolan ${ }^{2}$, K. Madden ${ }^{1}$, I. Thompson ${ }^{1}$ \\ ${ }^{1}$ Pharmacy, ${ }^{2}$ Medicine - Care of the Elderly, South Eastern Trust, Belfast, United Kingdom}

Background and objective: Osteoporotic fractures are a significant health issue in the aging population, resulting in increased morbidity, mortality and significant financial burden on the NHS. Medicines optimisation by a pharmacist can be a key intervention in reducing or preventing fractures and falls.

South Eastern Trust recently introduced a consultant led clinical pharmacy service for older people in intermediate care (IC). This service included a targeted, preventative approach to falls and fracture prevention.

This study aimed to determine the impact of the pharmacy team in optimising bone protective medication and in reducing falls risk.

Design: The IC team followed NOGG 2017 "Clinical guideline for the prevention and treatment of osteoporosis" to optimise bone protective medication or assess if the patient required referral for bone mineral density (BMD) assessment. A structured medicine review process was also used to reduce falls risk. Patients and/or carers were involved in all treatment decisions.

Over a 3 month period (early 2018) demographic, clinical and drug related data (including FRAX results, dietary calcium intake, pharmacist interventions) were collected on all patients admitted with fracture and/or falls to the IC unit.

Results: Data were collected for 48 patients (aged $84 \pm 8.1$ years; range $67-97$ years). $63 \%$ of all patients were admitted with fracture and $69 \%$ were admitted with a history of falls (that may or may not have resulted in fracture).

Risk assessments (FRAX) for those with a fracture or history of falls indicated bone protective treatment was advised in $69 \%$ of patients and a BMD scan in $31 \%$. For falls patients with no fracture $33 \%$ needed referral for BMD assessment and $67 \%$ required bone protective medication.

Decisions regarding bone protective medication were made for all patients reviewed including starting (44\% of patients), stopping $(23 \%)$ or that no medication was required or contraindicated $(40 \%)$. The most common pharmacist interventions were initiating Vitamin $\mathrm{D}$ replacement $(86 \%$ of patients either deficient or insufficient) and stopping calcium supplementation (64\%). 2 patients declined treatment with a bone protective medication as part of a shared decision making process.

A medication review was performed for all patients admitted with falls or history of falls $(n=33)$ resulting in 28 falls-associated medicines being stopped.

Conclusion: The introduction of the intermediate care pharmacy team has had a positive impact in falls and fracture prevention and in delivering a service to meet the Medicines Optimisation Quality Framework standards.

Disclosure of Interest: None Declared.
HP-PC088: Pharmacist led medication review of older people with an aim to deprescribing within the hospital setting

\section{J. Magee ${ }^{1, *}$, M. McManus ${ }^{1}$}

${ }^{1}$ Pharmacy, Northern Health And Social Care Trust, Antrim, United Kingdom

Background and objective: Due to the busy nature of ward working, medication review is not always possible as this can be a time consuming process. Polypharmacy can lead to drug interactions, adverse drug reactions, prescribing cascade, decreased quality of life, decreased cognition, higher costs and reduced adherence. This service aims to target high risk patients and perform a medication review as an inpatient, with a view to deprescribing where possible. The aim of deprescribing is withdrawal of inappropriate medications to reduce potential harm and the medication burden. Tools available to aid medication review include the following: Beers Criteria for Potentially inappropriate medication use in older adults, A Guide to Support Medication review in Older People produced by NIPOP and STOPP/START Criteria.

Design: A Band 8a Care of Older Person Pharmacist reviewed medications once weekly between October and December 2017. Data collected included patients hospital number, age, number of medications taken and the interventions made. Any changes made were graded using the Eadon scale and potential cost avoidance calculated using the ScHARR model.

Results: Sixty-eight patients had medication reviewed and the average age of the patient was $79.9 \pm 9.9$ years. The average number of medications taken per patient was $14.04 \pm 6.04$. The total number of interventions was 201 and of these 149 were medications discontinued. This resulted in an average number of interventions per patient of 2.96 and the average number of medications discontinued per patient was $2.2 \pm 1.58$. There were 3 interventions graded as 5 and 196 interventions graded as 4 using the Eadon scale. The results show a potential cost avoidance of $£ 14,879$ to $£ 33,864$ for the 68 patients reviewed during the 3 month period.

Conclusion: Medication review with the aid of available tools resulted in inappropriate medications being discontinued. Pill burden was reduced which translates to significant economic benefit to the NHS and potential reduction in patient harm.

Disclosure of Interest: None Declared.

HP-PC089: To explore, using qualitative studies, the perception and experiences of hospital clinical pharmacists in relation to provision of optimal and suboptimal pharmaceutical care

A. McLean ${ }^{1, *}$, V. Paudyal ${ }^{2}$, D. Stewart ${ }^{3}$, C. Souter ${ }^{1}$, E. Rankine ${ }^{1}$

${ }^{1}$ Pharmacy, NHS Lothian, Edinburgh, ${ }^{2}$ School of pharmacy, University of Birmingham, Birmingham, ${ }^{3}$ School of pharmacy and life sciences, Robert Gordon University, Aberdeen, United Kingdom

Background and objective: A qualitative study was undertaken to explore the concept of suboptimal pharmaceutical care. This study aimed to explore the perceptions and experiences of hospital clinical pharmacists in relation to provision of optimal and suboptimal pharmaceutical care .

Design: A qualitative study design was adopted. Focus groups were conducted with purposively selected clinical pharmacists of different bands from five NHS Lothian pharmacy departments for the initial phase of the research. The output from the focus groups was obtained using statements written onto post-it notes and placed on a flipchart by participants; audio recording was also used to obtain exemplar commentary from the participants that underpinned the written statement. 
One to one in-depth interviews were carried out with a number of the focus group participants. Interviews were audio recorded and transcribed. Interviews were semi-structured around the theoretical domains framework (TDF) domains, exploring participants experiences and perception of suboptimal pharmaceutical care. Interviews were carried out until data saturation was achieved with no new themes emerging. All data were anonymised.

Results: A total of 20 participants took part in the focus groups. They generated 78 statements that were mapped against TDF. Mapping occurred against 12 of the 14 domains. The majority were in the domain 'environmental context and resources'. This was exemplified by statements like "no clear area to record medicines reconciliation" and underpinned by comments like "there's just no consensus". Interviews, carried out with 10 participants, will be mapped to the TDF and descriptive statements will be used to describe the behavioural determinants of participants when providing or experiencing suboptimal pharmaceutical care.

Conclusion: Suboptimal pharmaceutical care was perceived by participants in this study as being seen as describing when care as planned could not be provided due to a range of factors. These factors have been successfully mapped against the theoretical domains framework.

Disclosure of Interest: None Declared.

HP-PC092: The development of a trust medicines adherence service for older people

R. Miller ${ }^{1,2, *}$, A. Friel ${ }^{1}$, C. Darcy ${ }^{1}$, H. McKee ${ }^{3}$, K. Miller $^{4}$, J. Agnew ${ }^{5}$, A. Cunningham ${ }^{3}$, J. Patterson ${ }^{4}$, J. McGennity ${ }^{5}$, S. Johnston ${ }^{6}$

${ }^{1}$ Pharmacy, Western Health and Social Care Trust, Londonderry, ${ }^{2}$ Medicines Optimisation Innovation Centre, ${ }^{3}$ Pharmacy, Northern Health and Social Care Trust, Antrim, ${ }^{4}$ Pharmacy, South Eastern Health and Social Care Trust, Belfast, ${ }^{5}$ Pharmacy, Southern Health and Social Care Trust, Craigavon, ${ }^{6}$ Pharmacy, Belfast Health and Social Care Trust, Belfast, United Kingdom

Background and objective: The Medicines Optimisation in Older People (MOOP) Northern Ireland network have developed a trust based medicines adherence specialist pharmacist led case management care pathway. The objectives are to embed and refine this pathway whilst also establishing outcomes.

Design: Under mentorship of consultant pharmacists, adherence pharmacists (one per trust) established the service. Pharmacists accept referral from trust staff via completion of a regional adherence assessment tool. They conduct comprehensive adherence assessments at patients' homes where they: determine medication appropriateness [using the Medication Appropriateness Index, (MAI)]; action clinical interventions which are Eadon self-graded (a scale of one to six where $\geq 4$ indicates improved quality of patient care); and provide/ recommend adherence solutions. They case manage patients for approximately 21 days.

Results: Data have been collected on 59 patients (aged $81.0 \pm 7.3$ years) across four trusts seen since January 1st 2018. Referrals have mainly come from social workers $(n=18)$, hospital pharmacists $(n=16)$ and occupational therapy $(n=8)$. Patients were case managed for an average of 25 days with a total of 320 solutions recommended/provided. Fifty-six solutions required liaison with the GP. One hundred and fifty-six clinical interventions were conducted with $82.7 \%$ Eadon self-graded $\geq 4$. The number of drugs taken reduced $(10.7 \pm 4.6$ vs $9.7 \pm 3.8$, Wilcoxon Signed Rank test, $p=0.014$ ) and appropriateness of prescribing improved (MAI Score $15.9 \pm 14.9$ vs $4.4 \pm 5.1$, Wilcoxon Signed Rank test, $p<0.001$ ) upon completion of case management.
Conclusion: The service is still embedding but preliminary results demonstrate positive and encouraging outcomes with future data collection also aiming to establish whether use of Monitored Dosage Systems or formal medicines management packages have been prevented.

Disclosure of Interest: None Declared.

HP-PC093: Analysis of first prescriptions of biosimilar etanercept

R. Morón ${ }^{1}$, P. Nieto Gómez ${ }^{1}$, C. Garcia Fernandez ${ }^{1}$, R. Alvarez Sanchez ${ }^{1, *}$

${ }^{1}$ Farmacia Hospitalaria, Hospital Universitario Clinico San Cecilio, Granada, Spain

Background and objective: To analyze preliminary experiences and the evolution of patients who have been administered etanercept biosimilar.

Setting and method: Retrospective observational study conducted in a third level hospital. From April 2017 to April 2018 the outpatients data were obtained from dispensing records.

Main outcome measures: Patients under treatment with biosimilar etanercept were collected since it was included in the pharmacotherapeutic guide of the hospital. Subsequently, all clinical reports were reviewed, including the service, indication, age, sex, initiation or switching and adverse events produced during the treatment with the studied drug.

Results: Thirty patients were registered during the study period, the average age was 50 years, 56.7 were women. $96.7 \%$ of the prescriptions were made by rheumatologists. The indications for which the drug was prescribed were: 10 ankylosing spondylitis, 9 psoriatic arthritis, 1 psoriasis and 10 rheumatoid arthritis. The average administration of the biosimilar drug is 25 vials. Of the 30 patients, switching was performed to 3 of them and 5 patients were changed from another biological therapy. The other 22 started the biological therapy with biosimilar etanercept. In only one case the biosimilar had to be suspended by reaction at the injection point.

Conclusion: Once the biosimilar drugs are commercialized, pharmacovigilance measurements must be performed in order to demonstrate in the general population what has been demonstrated in the comparative clinical trials. In our study we can conclude that there has been no adverse event that could be related to the change of drug. We must continue analyzing these types of practices so that, in the future, we can confirm in the daily practice that biosimilar drugs are equivalent to their reference drug in their safety and efficacy profile. Disclosure of Interest: None Declared.

HP-PC095: Evaluation of the use of dabigatran etexilate in patients with non valvular atrial fibrillation who undergo an ablation of the isthmus

P. Nieto Gómez ${ }^{1}$, R. Álvarez Sánchez ${ }^{1, *}$, A. Rodríguez Delgado ${ }^{1}$, M. Valle Corpas ${ }^{1}$, D. Blánquez Martínez ${ }^{1}$

${ }^{1}$ Farmacia Hospitalaria, Hospital Clinico San Cecilio, Granada, Spain

Background and objective: It is essential to perform anticolagulation in patients with non-valvular atrial fibrillation, who are going to undergo ablation of the isthmus, to prevent thromboembolic strokes in the peri-intervention period. The aim of this study is to analyze the use of dabigatran etexilate in this indication.

Setting and method: For this study, we made a search on the clinical records of 37 patients with non-valvular atrial fibrillation who underwent ablation of the isthmus and were treated with dabigatran etexilate, between January 2017 and December 2017. 
Main outcome measures: Demographic data (age, sex) were recorded., number of days of anticoagulation before and after the procedure, and both bleeding risk factors (age, use of antiaggregants, hypertension, alcoholism, hemorrhagic history, renal, hepatic insufficiency) and thrombotic (age, sex, hypertension, congestive heart failure, diabetes mellitus, history of thrombotic episodes, vascular disease). The thrombotic (CHA2DS2-VASC) and hemorrhagic (HASBLED) risks were calculated with these data.

Results: All patients included in the study were anticoagulated with dabigatran etexilate at a dose of $150 \mathrm{mg}$ twice daily. $81 \%$ of the patients were men. The median age of the patients was 51 years (43-58). The median days of anticoagulation was 90 (60-121). 54\% of the patients had a risk CHA2DS2-VASC of 0 , and $43 \%$ of patients risk 1 . Regarding the hemorrhagic risk, only one patient had a HASBLED $>2.40 .5 \%$ of the patients presented a CHA2DS2-VASC $<2$ and were more than 90 days anticoagulated. For this group, the median days of anticoagulation was 300 (120-405), and 279 (120-405) after intervention. Of them, 33.33\% are still under treatment. Within this group, $46 \%$ had CHA2DS2-VASC of 0 , and they were 150(120-300) days anticoagulated.

Conclusion: According to the guidelines, anticoagulation must be maintained during a minimum of 8 weeks after this procedure in all patients. Anticoagulation must be adapted to the thrombotic and hemorrhagic risk of each patient and not depending on the possible success or failure of the intervention. That is why we think that maintaining anticoagulation in patients with a CHA2DS2-VASC risk lower than 2 is not justified and patients are exposed to possible adverse events with no additional benefit.

Disclosure of Interest: None Declared.

\section{HP-PC096: Standardisation to minimise delivery time} of personalised analgesia in hospitals

\section{V. Jovanovic ${ }^{1}$, S. E. Ørnes ${ }^{2, *}$, H. Thoresen ${ }^{1}$, J. U. Brænden ${ }^{2}$ \\ ${ }^{1}$ School of Pharmacy, University of Oslo, Oslo, ${ }^{2}$ Hospital pharmacy enteprises, South Eastern Norway, Akershus, Norway}

Background and objective: Patient-controlled analgesia (PCA) is important in pain management in palliative cancer care. Various analgesic mixtures for PCA are prepared at the hospital pharmacy for the individual patient. Production is time consuming and the shelf life of the product is limited. Product with standardized content could be manufactured as off-the-shelf product with longer shelf life. The purpose of the study was therefore to map prescribing practices of PCA and to see whether standardized drug contents are a possibility at the Palliative Unit at Akershus University Hospital HF (Ahus) and Hospice Lovisenberg in Oslo.

Setting and method: The project was performed as a quantitative cross-sectional survey applying a questionnaire with manly closed questions, among the palliative physicians at the two hospitals. The responses were analysed descriptively. In addition, all orders to the hospital pharmacy of PCA from these centers were registered.

Main outcome measures: The degree of similarity in the answers from the questionnaire.

Results: Nine of the eleven physicians answered the questionnaire. The physicians at Ahus answered that their prescribing practice were influenced by the delivery time, in opposite to the physicians at Hospice Lovisenberg. The physicians from Ahus rarely prescribed mixtures and wanted the substances in separate cassettes to allow for individual dose adjustment. Physicians from Hospice Lovisenberg often prescribed mixtures of substances into one cassette to make it easy to handle for the patient. All agreed on that they lacked of-theshelf morphine cassettes at higher concentrations, 20 and $40 \mathrm{mg} / \mathrm{ml}$ and oxycodone cassettes at higher concentrations, $10 \mathrm{mg} / \mathrm{ml}$ and $20 \mathrm{mg} / \mathrm{ml}$.
Conclusion: This study indicate that it is possible to standardize certain active substances such as morphine and oxycodone as off-theshelf products. For the more complex drug mixtures, the prescribing traditions are different and it may be difficult to agree on standard concentrations. There may also be a need to change prescribing traditions to achieve further standardization.

Disclosure of Interest: None Declared.

HP-PC097: A like antimicrobial stewardship—where do we stand in Austrian hospitals?

\author{
E. Past ${ }^{1, *}$, D. Müller ${ }^{2}$, S. Mair ${ }^{3}$, U. Porsche ${ }^{1}$ \\ ${ }^{1}$ Landesapotheke Salzburg, Salzburg, ${ }^{2}$ Hanusch Krankenhaus, \\ Vienna, ${ }^{3}$ Tirol Kliniken, Innsbruck, Austria
}

Background and objective: To determine the current stage of implementation of antimicrobial stewardship in Austrian hospitals.

Design: Literature review; development of a set of survey questions by a team of four clinical pharmacists and one non-pharmacist; dissemination via an online tool (SurveyMonkey ${ }^{\circledR}$ ) to the mailing list of the Austrian Association of Hospital Pharmacists to all chief hospital pharmacists; analysis of the survey data.

Results: The response rate was $89.5 \%(\mathrm{n}=34)$ with participation of chief pharmacists from every Austrian state. Noteworthy is the fact that only $13.8 \%(n=275)$ of Austrian hospitals have an on-site pharmacy. Nearly half of the resonding hospitals were of medium size with $300-700$ beds $(47.1 \%, \mathrm{n}=16)$ and $29.4 \%(\mathrm{n}=10)$ were large hospitals with $>1000$ beds. Only four hospitals employed more than 10 pharmacists (11.8\%), 14 hospitals less than five pharmacists. Antimicrobial stewardship teams (AMT) were established in $70.1 \%$ $(n=24)$ of the hospitals. In every AMT at least one pharmacist was on board. Data on local antimicrobial resistance rates was routinely gathered on almost all sites $(94.1 \%, \mathrm{n}=32)$; antimicrobial usage data was available in $64.7 \%(\mathrm{n}=22)$ with 15 hospitals participating in a transregional surveillance programme. The majority of hospitals had local antimicrobial guidelines $(88.2 \%, \mathrm{n}=32)$, mostly electronically available $(73.5 \%, \mathrm{n}=25)$ and supported their staff with continued education in infectious diseases $(76.5 \%, \mathrm{n}=26)$. Preauthorisation for certain antimicrobials was used by $76.5 \%(n=26)$ of AMTs. Early switch from IV to oral options was promoted via guidelines in $32.4 \%$ $(n=11)$ of hospitals. In $14.7 \%(n=5)$ of hospitals, routine bedside infectious disease consultation was provided by the AMT. Audit and feedback strategies were used in $23.5 \%(n=8)$ of hospitals.

Conclusion: We achieved an excellent response rate; antimicrobial stewardship seems to be a hot topic among chief pharmacists in Austrian hospitals. Resistance and usage data of antimicrobials were widely available in all contacted hospitals. Front-end antimicrobial stewardship strategies such as local guidelines, restriction of certain antimicrobials and educational programmes were commonly implemented. In $70.6 \%$ of the included hospitals, an AMT was in office. There is need to invest more in back-end or postprescription strategies to improve antimicrobial prescribing, ideally led by an AMT. Strategies such as bedside consultation and audit-feedback interventions could further improve the direct impact of antimicrobial stewardship on patient care.

Disclosure of Interest: None Declared.

HP-PC098: Candidate preferencing of their future employers in Health Education England pre-registration pharmacist recruitment scheme: an exploration of candidate behaviour and associated factors in their decision making

L. McEwen-Smith ${ }^{1}$, G. Fleming ${ }^{1}$, C. Hirsch ${ }^{2}$, S. Buckley ${ }^{2}$, J. Ward ${ }^{2}$, S. Haque ${ }^{2}$, V. Paudyal ${ }^{3, *}$ 
${ }^{1}$ Health Education England, London, ${ }^{2}$ University Of Birmingham, Birmingham, United Kingdom, ${ }^{3}$ School of Pharmacy, University of Birmingham, Birmingham, United Kingdom

Background and objective: A centralised system for recruiting to all hospital preregistration pharmacist training and many community pharmacy posts based in England and Wales was introduced in 2017. Applicants submit a single application and are allocated their highest preferenced training place, based on their performance in selection centres. This evaluation aimed to explore applicant preferencing of their future employers and factors influencing their behaviour.

Setting and method: This evaluation utilises a mixed method study design, with data collected as phased investigation. This involves cross-sectional online survey of all students in England and Wales undertaking Master of Pharmacy Year 4 along with semi-structured qualitative focus groups and telephone interviews. Statistical analysis of questionnaire data is being undertaken, involving both descriptive and inferential analysis. The framework technique is being used to analyse the qualitative data generated from the focus groups and telephone interviews.

Main outcome measures: Factors affecting applicant preferencing behaviours and perceptions of the impact of their preferencing choices on their training place outcome.

Results: Data is currently being collected, due to complete in May 2018. Preliminary analysis has been undertaken based on the responses from 200 students. Overall, respondents were satisfied with the preferencing process and their approach to selecting training places. More than half of respondents indicated that long-term career aspirations, proximity to permanent or intended long-term home and information made available by employers about their training programme were the most influential factors in their selection of training places. Proximity to university and existing relationships with employers were rated as having no influence at all on decision making, by over half of respondents. A total of $83.5 \%$ of respondents indicated their satisfaction with their training place offer outcome. Information available from employers, particularly community pharmacy employers, about their training programme and training place location were the most cited reasons for respondent dissatisfaction, as noted through qualitative results.

Conclusion: Findings offer valuable insight into influencing factors underpinning student preferencing strategies and the impact of this on outcomes. These findings will be used to directly inform future employer communication and operational processes with the aim of increasing applicant satisfaction and retention within training places. Disclosure of Interest: None Declared.

HP-PC099: Personalised care against hepatitis C: impact of HIV and Chronic Kidney Disease comorbidities on treatment choices, clinical outcomes and relevant drug-drug interactions

S. Ali ${ }^{1}$, E. Lougher ${ }^{2}$, T. Ur-Rehman ${ }^{3}$, D. Mutimer ${ }^{2}$, S. Haque ${ }^{1}$, V. Paudyal ${ }^{1, *}$

${ }^{1}$ University Of Birmingham, ${ }^{2}$ NHS University Hospitals Birmingham, Birmingham, United Kingdom, ${ }^{3}$ Quaid-i-Azam University, Islamabad, Pakistan

Background and objective: High prevalence of comorbidities add significant risk to sub-optimum clinical outcomes and envisage potential interaction with concomitant medication. HIV infection and chronic kidney disease (CKD) are two important co-morbidities of Hepatitis C. This study aimed to explore and compare the treatment choices and clinical outcomes in HCV patients with/without comorbidities of HIV and CKD.

Setting and method: This retrospective study included patients who received hepatitis C treatment between July 2015 and January 2018, within a Hepatitis C operation Delivery Network in the West Midlands, England. Patients with hepatitis $\mathrm{C}$ virus (HCV) mono-infection, those coinfected with HIV or comorbid with CKD were included. Data on the demographics, baseline viral load, genotype, liver staging, previous medications, treatment choices as per run rate card of NHS England and concomitant medications were extracted using validated spread sheet.

Main outcome measures: Treatment success as measured in terms of sustained virological response (SVR 12) and assessment of drug-drug interactions (DDIs) with the available Hepatitis $\mathrm{C}$ regimens.

Results: Of a total of $314 \mathrm{HCV}$ patients, 154(49.0\%) were without comorbidity, 124(39.5\%) were coinfected with HIV and 36(11.5\%) had comorbidity of CKD. Mean (SD) age was $49.67( \pm 12.05)$ years, $234(74.5 \%)$ were male and $180(57.3 \%)$ were white. One hundred and ninety-three $(61.5 \%)$ were non-cirrhotic and $199(63.4 \%)$ were treatment naive. Genotype 3/3a was the most prevalent 111(35.4\%) and $50(15.9 \%)$ of HIV infected were PWIDs. The average number of concomitant medicines per patient were 2.78 (range 0-12) in monoinfected, 3.57 (range 0-16) in HIV and 6.8 (range 0-13) in CKD patients. Of total $217(69.11 \%)$ were successfully cured to SVR, $3(0.96 \%)$ stopped early, $11(3.50 \%)$ relapsed/failed, $73(23.24 \%)$ awaiting results, $05(1.59 \%)$ not on database, $1(0.75 \%)$ died (sadly) and $4(1.27 \%)$ were lost to follow up. Of total co-prescriptions, $20(12.99 \%)$ potential DDIs were identified in HCV (mono), 94(75.81\%) in HIV and 24(66.67\%) in CKD prescriptions for which treatment modifications were made.

Conclusion: Treatment adjustment conducted as per the comorbidities, genotype and previous treatment experience and with consideration of drug-drug interactions provides a successful outcome in HCV patients co-morbid with HIV or CKD.

Disclosure of Interest: None Declared.

HP-PC100: Appropriateness of antibiotic prescribing in community-acquired pneumonia at a tertiary hospital

D. Peña-Lorenzo 1,*, N. Rebollo-Díaz ${ }^{1}$, M. Martín-Gil ${ }^{1}$, M. Beunza-Sola ${ }^{1}$, R. Fernández-Caballero' ${ }^{1}$, R. Martín-Muñuz ${ }^{1}$, B. Blanco-Serrano ${ }^{1}$, M. J. Otero-López ${ }^{1}$

${ }^{1}$ Pharmacy, Complejo Asistencial Universitario de Salamanca, Salamanca, Spain

Background and objective: Patients hospitalized with communityacquired pneumonia (CAP) have better outcomes if they are treated with empirical antimicrobial therapy according to the Infectious Diseases Society of America (IDSA)/American Thoracic Society (ATS) guidelines for CAP within the first $24 \mathrm{~h}$ of hospitalization (Arnold et al., 2009). The aim of the study was to assess the appropriateness of antibiotic prescription for CAP based on local guidelines which have been adapted from IDSA/ATS recommendations.

Design: Observational retrospective study carried out at a tertiary hospital that included non-intensive care unit patients diagnosed with CAP from November 21, 2017 to January 12, 2018. The following data were collected from medical records: age, sex, antimicrobial treatment and antibiogram.

To assess the appropriateness of antibiotic prescription, the following criteria were analyzed: (1) correct empirical antibiotic selection according to the guidelines, (2) antibiotic de-escalation and, (3) early switch from intravenous to oral route before day 3. Empirical therapy was considered appropriate when $\beta$-lactam \pm macrolide or respiratory fluorquinolone were the first-line treatment if Pseudomonas or MRSA were not a concern, or amoxicillin/clavulanic acid when bronchoaspiration was suspected. Early switch was considered as appropriate when another oral medication was administered and the criteria for clinical stability were present (temperature $<37.8^{\circ} \mathrm{C}$, 
heart rate $<100 x$ ', systolic blood pressure $\geq 90 x^{\prime}$, oxygen saturation $\geq 90 \%$ and respiratory rate $\left.<24 x^{\prime}\right)$.

Results: Included were 68 patients (40 men) with a median (range) age of 81 years (36-95). Only $51 \%$ of the patients received antibiotic treatment according to local guidelines. Treatments considered to be appropriate were: ceftriaxone + azithromycin (5.9\%), levofloxacin (25\%), amoxicillin/clavulanic acid (16.2\%), and ceftriaxone $(4.4 \%)$. The most frequent non-appropriate treatment was levofloxacin + ceftriaxone $(22.1 \%)$.

In $23.5 \%$ of patients, a microorganism was identified. As expected, Streptococcus pneumoniae was the most frequent isolated microorganism (18.8\%). De-escalation was effectuated according to the antibiogram in $27 \%$ of patients.

At day 3, it was not possible to assess the respiratory rate because it is not commonly measured in our hospital's clinical practice. The rest of the criteria for clinical stability were met in $51.4 \%$ of patients who were receiving another medication by oral route, but only in $54.4 \%$ of them early switch was performed.

Conclusion: A high rate of antibiotic prescription for CAP patients is not appropriate, since in half of patients the empirical treatment selection was not made according to local guidelines. Moreover, we observed that antibiotic de-escalation and switch from oral to intravenous administration did not follow established recommendations. These results show a need for educational interventions in the context of our antibiotic stewardship program.

Disclosure of Interest: None Declared.

\section{HP-PC102: Isoleucine and valine: survey on the uses in French} hospitals

\section{R. Rasamison ${ }^{1, *}$, J.-M. Alili ${ }^{1}$, M.-P. Berleur ${ }^{1}$, M.-C. Husson ${ }^{1}$}

${ }^{1}$ Regulatory, Pharmaceutical and Medical Department, AGEPS, Paris, France

Background and objective: Maple Syrup Urine Disease (MSUD) is a rare disease which could be fatal when neither diagnosed nor treated quickly. Its treatment consists in dietary leucine restriction, restriction of branched-chain amino acids (AA) e.g. leucine isoleucine and valine, and pertinent adapted supplementation with isoleucine and valine to achieve sustained protein synthesis in tissues during metabolic crisis.

AGEPS manufactures and distributes Isoleucine (ILE) $50 \mathrm{mg}$ capsules and Valine (VAL) $50 \mathrm{mg}$ capsules, two hospital preparations.

The aim of this work was to study their current therapeutic uses in French hospitals.

Design: The survey was conducted in April-December 2017 in 17 French hospitals which were the largest customers of ILE and VAL during the past 3 years. Pharmacists and clinicians from Endocrinology and Paediatric wards were contacted by phone or email; a standardized questionnaire was used.

The questionnaire had 6 opened and closed questions concerning the uses, the doses, the number of patients treated in 2016, and the alternative treatments.

Results: Eight pharmacists and 4 clinicians from 8 hospitals, including 4 reference centers for metabolic diseases, answered.

Two therapeutic uses were identified for both AA administrated at various doses:

- MSUD (65 patients): 5-20 mg/bw/d in newborn, $600-1200 \mathrm{mg} / \mathrm{d}$ in adult;

- propionic and methylmalonic acidemia (3 patients): 150-400 mg/ $\mathrm{d}$ in children.

Propionic and methylmalonic acidemia are inborn errors of metabolism characterized by an accumulation of propionic acid and methylmalonic acid in plasma, urine and other body fluids. This accumulation is due to the deficiency of enzymes (propionyl-CoA carboxylase and methylmalonyl-CoA mutase). Clinical manifestations can include: metabolic acidosis, seizure, cutaneous lesions, severe neurological handicap (development delay, encephalopathy or movement disorder), coma or death. ILE/VAL supplementation is integrated in the standard therapy to achieve normal development and to prevent episodes of metabolic decompensation.

Concerning the alternative treatment, it consists in capsules prepared in community or hospital pharmacies. Mixture of essential AA was also used, but they do not always contain enough ILE and VAL regarding to patients' needs during metabolic crisis.

Conclusion: According to this survey, ILE and VAL are essential to treat MSUD.

ILE combined with VAL are indispensable to treat propionic and methylmalonic acidemia in order to compensate for a lack of these two amino acids.

Observed doses vary with the therapeutic uses and the severity of the symptoms. They are consistent with literature data.

This survey has now to be completed with the total number of treated patients in France to identify a target population.

Disclosure of Interest: None Declared.

HP-PC103: Arginine and citrulline: survey on the uses in French hospitals

R. Rasamison, ${ }^{1, *}$, J.-M. Alili ${ }^{1}$, M.-P. Berleur ${ }^{1}$, M.-C. Husson ${ }^{1}$

${ }^{1}$ Regulatory, Pharmaceutical and Medical Department, AGEPS, Paris, France

Background and objective: Arginine (ARG) $6.25 \%$ (w/v) solution for infusion, and Citrulline (CIT) $500 \mathrm{mg}$ capsules are two hospital preparations manufactured and distributed by AGEPS. ARG and CIT are amino acids both used in hyperammonemia due to urea cycle disorders (UCD), a category of rare disease. UCD can be fatal when neither diagnosed nor treated quickly.

ARG is also used for Growth Hormon (GH) stimulation test in adults and children.

The aim of this work was to study their therapeutic uses, protocols and doses, in French hospitals.

Design: The survey was conducted in April-December 2017 in 19 French hospitals which were the largest customers of ARG and CIT, respectively during the past 3 and 2 years. Pharmacists and clinicians from Endocrinology, Paediatric and Gastroenterology wards were contacted by phone or email; a standardized questionnaire was used. The questionnaire had 9 opened and closed questions concerning the uses, the doses, the number of patients treated in 2016, and the potential alternative treatment.

Results: Sixteen pharmacists and 8 clinicians from 13 hospitals, including 4 reference centers for metabolic diseases, answered the questionnaire.

For ARG, 5 therapeutic uses were identified:

- UCD (260 patients): 50-300 mg/bw/d given in 3 divided doses in children and 3-6 g/d in adults;

- GH stimulation test: a standard protocol was used with $0.5 \mathrm{~g} / \mathrm{bw} /$ test, maximum $25 \mathrm{~g}$ per test;

- MELAS (Mitochondrial Encephalomyopathy, Lactic Acidosis, and Stroke-like episodes) syndrome: bolus of intravenous arginine ( $500 \mathrm{mg} / \mathrm{bw}$ for children or $10 \mathrm{~g} / \mathrm{m}^{2}$ body surface area for adults) within 3 first hours;

- severe metabolic alkalosis (2 patients): dose dependeded on metabolic alkalosis severity;

- home parenteral nutrition in adult chronic intestinal failure: $2 \mathrm{~g}$ ARG/bag, 2-6 bags/week. 
One alternative treatment was identified for UCD and severe metabolic alkalosis (ARG 21\% solution for infusion).

For CIT, one use was identified:

- UCD (240 patients): 50-300 mg/bw/d given in 3 divided doses for children and 3-6 g/d for adults.

Alternative treatment could consist in capsules of CIT prepared out by community or hospital pharmacist.

Conclusion: According to this survey, and as confirmed by the literature, CIT and ARG are essential to treat UCD.

Effectiveness should be evaluated before considering whether ARG is essential for MELAS syndrome, severe metabolic alkalosis and home parenteral nutrition to treat adult chronic intestinal failure.

Observed doses vary with the therapeutic uses and the severity of the symptoms. They are consistent with literature data.

This survey should be completed now with the total number of treated patients in France to identify a target population.

Disclosure of Interest: None Declared.

HP-PC105: Reduction of the duration of antibiotic therapy in community-acquired acute respiratory tract infections as one of the goals of Antimicrobial stewardship programs

S. Sadyrbaeva Dolgova ${ }^{1, *}$, B. Cancela-Díez ${ }^{1}$, P. Aznarte-Padial ${ }^{1}$, R. Alvarez ${ }^{1}$, C. Hidalgo-Tenorio ${ }^{2}$, J. Pasquau ${ }^{2}$

${ }^{1}$ Pharmacy, ${ }^{2}$ Infectious Diseases, Hospital Universitario Virgen de Las Nieves, Granada, Spain

Background and objective: Achieving a reduction of antibiotic exposure still remains the main objective of any antibiotic policy. There is a well-known relationship between the overall amount of antibiotic exposure and the development of bacterial resistance. Our objective was to assess the impact of interventions performed within an antimicrobial stewardship program to reduce the duration of antibiotic therapy in Community-acquired pneumonia (CAP) and Acute Chronic Obstructive Disease (CPOD).

Setting and method: A retrospective quasi-experimental before-after study performed in a tertiary university hospital. Data for the first cohort were collected in 2011 and those for the second cohort in 2017. The study included patients with CAP and CPOD in the Respiratory Department. The intervention consisted in two training sessions in the department and subsequent implementation of antibiotic duration follow-up through the Pharmacy department. Medians and interquartile range were calculated for quantitative variables and absolute and relative frequencies for qualitative variables. The relationship between quantitative variables with non-normal distribution (Kolmogorov-Smirnov test) was analysed with the non-parametric Mann-Whitney test.

Main outcome measures: Antibiotic duration and length of hospital stay (LOS).

Results: 201 patients were analysed in total: 101 in the 2011 cohort (before) and 101 in 2017 (after). In 2011: 81(80.2) were male, age $>70$ years: $57.4 \%$. CAP 53(52.5) and CPOD 48(47.5) patients. Patients were with respiratory failure 59(58.4\%), diabetes $22(21.8 \%)$, renal failure 6(5.9), heart failure $10(9.9 \%)$, 11(10.9) with severe immunodeficiency. Microorganisms were identified in 19(18.8\%) patients and 3 of them with bacteraemia. In 2017: 85(84.2\%) were male, age $>70$ years: $68.3 \%, 60(59.4)$ patients with CAP and $41(40.6 \%)$ with COPD. Patients were with respiratory failure $58(57.4 \%)$, diabetes $31(30.7 \%)$, renal failure $15(14.9)$, heart failure 27(26.7\%), 14(13.9) with severe immunodeficiency. 9 (8.9) patients with microorganisms identified and 3 of them with bacteraemia. The medians of duration of antibiotic therapy for CAP were 13(IQR 12-17) days in the 2011 cohort and 10.5(IQR 9-13) days in the 2017 cohort, $p<0.001$. For COPD, the medians were 12.5(IQR11-15) days and 9(7.5-11) days respectively, $p<0.001$. LOS for CAP were 9(IQR7-11) days in 2011 and 7(IQR5-8) days in 2017, $p<0.001$ and for COPD were 9(IQR7-11) days and $8(7-11)$ days, respectively, $p=0.265$.

Conclusion: Although more severely ill patients and with more comorbidities were included in the post-intervention phase, the reduction of duration was achieved, which has had a positive impact in length of hospital stay. However, for the achievement of optimal duration for this infections (less than 8 days), some additional interventions are needed.

Disclosure of Interest: None Declared.

HP-PC106: What is the knowledge of patients about their oral anticoagulant therapy?

P. Sagourin 1,*, F. Benamar', M. Le Barbu' ${ }^{1}$, E. Moutel ${ }^{1}$, F. Plassart ${ }^{1}$, M. Abderrahmane ${ }^{2}$, S. Fedy ${ }^{2}$, J.-L. Pons ${ }^{1}$, F. Lemann ${ }^{2}$

${ }^{1}$ Pharmacy, ${ }^{2}$ Quality, Centre Hospitalier Victor Dupouy Argenteuil, Argenteuil, France

Background and objective: Recently, the guidelines of French health authorities redefined the position of DOAs (Direct Oral Anticoagulants) and VKAs (antivitamin K) drugs. They are looked at with scrutiny because of an iatrogenic risk associated with their administration. A survey regarding oral anticoagulants was conducted to evaluate the knowledge of hospitalized patients about their chronic treatment.

Design: The questionnaire has been validated by the Drug delivery and monitoring system group. The survey was conducted during 2 months in 13 care units. The interviews were conducted by two practitioners, two pharmacists, two pharmacist residents, two quality risk managers. For the statistical analysis, the patients were stratified between VKAs and DOAs treatment, the Chi 2 test was used.

Results: Sixty patients were interviewed (30 in each group). For VKAs, fluindione is mainly prescribed $(\mathrm{n}=23)$. For DOAs, the most prescribed are apixaban $(n=13)$ and rivaroxaban $(n=11)$. Patients treated with VKAs are more familiar with the name of their drugs $\left(\mathrm{P}_{\mathrm{VKA}}=73 \%, \mathrm{P}_{\mathrm{DOA}}=30 \%, p<0.05\right)$ and the modality of administration $\left(\mathrm{P}_{\mathrm{VKA}}=80 \% \mathrm{P}_{\mathrm{DOA}}=57 \%, p=0.05\right)$. On the contrary, patients of each group are not familiar with the mechanism of action and the signs of bleeding. There is not significant difference between each group (respectively $\mathrm{P}_{\mathrm{VKA}}=57 \%, \mathrm{P}_{\mathrm{DOA}}=60 \%, p>0.05$ and $\mathrm{P}_{\mathrm{VKA}}=$ $\left.37 \%, \mathrm{P}_{\mathrm{DOA}}=40 \%, p>0.05\right)$. Paracetamol is more often cited as an analgesic treatment by patients treated by VKAs $\left(\mathrm{P}_{\mathrm{VKA}}=93 \%\right.$, $\left.\mathrm{P}_{\mathrm{DOA}}=53 \%, p<0.05\right)$. For the VKA group, the presence of a followup card is not significantly higher $\left(\mathrm{P}_{\mathrm{VKA}}=33 \%, \mathrm{P}_{\mathrm{DOA}}=17 \%\right.$, $p>0.05)$ and $67 \%$ of the patients are able to quote their target International Normalized Ratio.

Conclusion: The audit showed that patients treated by DOAs were less informed about their treatment. Following this, the information cards were retrieved from the DOAs boxes and sent to some care units. A patient information leaflet was created based on the model of one regional Drugs Observatory. The results of this audit will be presented in Drugs and Therapeutics committee and on the medical board of the hospital. The setting up of pharmaceutical interviews will be discussed with the practitioners for the patients who really need it. Disclosure of Interest: None Declared.

HP-PC108: Problems in usage of dietary supplements in elderly patients: a systematic review of case reports related to toxicity

S. L. Söylemez ${ }^{1 \text {,* }}$, Y. Gönen ${ }^{1}$, M. Sancar ${ }^{1}$, B. Okuyan ${ }^{1}$

${ }^{1}$ Clinical Pharmacy, Marmara University, İstanbul, Turkey 
Background and objective: The concerns related to toxicity raise with usage of dietary supplement, especially when concomitantly used with prescribed medications. Polypharmacy is more common in elderly patients. Moreover, elderly patients tend to exposure more toxicity than other age groups because of physiological changes seen in these patients. In this study, it is aim to review case reports in literature related with usage of dietary supplement resulted in toxicity in elderly patients.

Setting and method: Case reports, which related with patients at the age of 65 or older, were searched on Medline and PubMed databases before December 2017. Following keywords used to refine related case reports: 'toxicity' and 'dietary supplement'. Prescribed vitamin and mineral supplements were excluded if they were used in management of chronic disease such as niacin, vitamin D.

Main outcome measures: Case reports related with usage of dietary supplement resulted in toxicity in elderly patients.

Results: Sixty-six case reports in 42 articles, which is written English with accessible full text, were refined out of two hundred-sixty-three abstracts. Of them, $55 \%$ were women $(n=36)$. Mean age was $73.85 \pm 6.4$. Polypharmacy is seen in 10 out of 66 case reports. The most common dietary supplement related with toxicity was glucosamine sulphate $(\mathrm{n}=14 ; 21.21 \%)$, ginkgo biloba $(\mathrm{n}=3 ; 4.5 \%)$ and fish oil $(\mathrm{n}=3 ; 4.5 \%)$. The most common toxicities related with dietary supplements were drug-supplement interaction $(\mathrm{n}=10$; $15.1 \%)$, haemorrhage $(\mathrm{n}=8 ; 12.1 \%)$, dermatologic events $(\mathrm{n}=8$; $12.1 \%$ ), hepatotoxicity $(\mathrm{n}=7 ; 0.6 \%)$. It is reported that glucosamine sulphate, ginkgo biloba, fish oil, muscadine grape and Artemisia absinthium caused bleeding when concomitantly used with warfarin that is the most common medication involved in drug-supplement interaction in the present study. In the majority of the cases, it is seen that after withdrawing dietary supplements, toxicities disappears. Therefore, it is concluded that dietary supplements causes emergence of toxicities.

Conclusion: It has been observed that toxicities are mostly associated with interactions between medicines. The literature review demonstrates that elderly patients need pharmacists who are specialists about medicines and supplements to protect their health and enhance quality of life.

Disclosure of Interest: None Declared.

HP-PC109: Prescribing appropriateness of direct oral anticoagulants in a university hospital and determination of risk factors that contribute to inappropriate prescribing

\section{S. Moudallel ${ }^{1, *}$, S. Steurbaut ${ }^{1}$, P. Cornu ${ }^{1}$, A. G. Dupont ${ }^{1}$ \\ ${ }^{1}$ Clinical Pharmacology \& Pharmacotherapy, UZ Brussel, Brussels, Belgium}

Background and objective: Appropriate dosing of direct oral anticoagulants (DOACs) is required to avoid under- and overdosing that may precipitate strokes or thromboembolic events and bleedings, respectively. The objective of this study was to analyze the appropriateness of DOAC dosing according to the summaries of product characteristics (SmPC). In addition, determinants for inappropriate prescribing were investigated.

Setting and method: Retrospective cohort study of all hospitalized patients treated with a DOAC (rivaroxaban, dabigatran or apixaban) between 1/1/2016 and 31/12/2016 in the UZ Brussel (a 721-bed tertiary hospital). All patients aged $\geq 60$ years with at least one DOAC intake during their hospital stay were included. The Chi square test was used to evaluate differences between the DOACs. Binary logistic regression analysis was performed to assess determinants for inappropriate prescribing.

Main outcome measures: The rate of inappropriate DOAC prescriptions and associated risk factors.
Results: In total, 998 hospitalizations were analyzed representing 777 unique patients. Inappropriate dosing occurred in total for $25.7 \%$ of hospitalizations with $26.2,22.3$ and $29.5 \%$ for dabigatran, rivaroxaban and apixaban, respectively $(p=0.084)$. Underdosing, according to the SmPC, was most prevalent for apixaban $(24.8 \%)$ compared to dabigatran $(16.6 \%)$ and rivaroxaban $(12.6 \%), p<0.001$. All underdosed cases were subsequently evaluated using the European Heart Rhythm Association (EHRA) 2018 atrial fibrillation guidelines. In $67.7 \%$ (apixaban), 29.2\% (dabigatran) and $52.7 \%$ (rivaroxaban) of underdosed DOAC cases according to the SmPC, the dose would be considered appropriate according to EHRA.

Overdosing was observed in $4.7 \%$ (apixaban), 9.7\% (dabigatran) and 9.8\% (rivaroxaban) of hospitalizations.

When considering all DOACs together, decreased renal function $(p=0.001)$ and being hospitalized with a DOAC as prehospital medication ( $p=0.004)$ were identified as determinants for inappropriate prescribing. A subgroup analysis for dabigatran revealed only decreased renal function $(p=0.005)$ as risk factor for inappropriate dosing. For rivaroxaban, use of the reduced dose $(p=0.003)$ and the indication for which this DOAC was used $(p=0.009)$ were found to have an influence. For apixaban, the reduced dose $(p<0.001)$, decreased renal function $(p=0.001)$ and being hospitalized with this DOAC as prehospital medication $(p=0.049)$ played a role in inappropriate prescribing.

Conclusion: Inappropriate prescribing of DOACs is frequent with underdosing being the most common drug related problem when using the SmPC as reference. Application of the EHRA guidelines resulted in substantially less cases of underdosing indicating that these guidelines are more tolerant regarding the use of reduced DOAC doses. Analysis of determinants of inappropriate prescribing yielded insights in the risk factors associated with inappropriate DOAC prescriptions. These factors can be taken into account by clinical pharmacists and/or clinical decision support systems to increase the level of appropriate DOAC therapies.

Disclosure of Interest: None Declared.

\section{HP-PC110: Identifying patients at risk of QTe prolongation} as a part of Antimicrobial Stewardship Program

\section{E. Tannous ${ }^{1, *}$, K. Amarney ${ }^{2}$}

${ }^{1}$ Pharmacy, Hillel Yaffe Medical Center, Haifa, ${ }^{2}$ Pharmacy, Hillel Yaffe Medical Center, Hadera, Israel

Background and objective: QT interval prolongation is a repolarization abnormality which may increase the risk for torsades de pointes (Tdp) and sudden cardiac death (SCD). Various medications have been identified as prolonging the QT interval, requiring monitoring. However, monitoring the QT interval presents two important challenges: accurate measurement and correction to heart rate (QTc). Overestimation of the QTc interval in the case of ventricular conduction delay (VCD) may lead to unwarranted discontinuation or avoidance of first line medications. Conversely, underestimation of the QTc interval may lead to increased risk of TdP and SCD.

Many antimicrobials increase the QTc interval and may be prescribed for patients hospitalized due to an infectious disease. We sought to investigate the risk for QTc prolongation in patients for whom a consultation by an infectious disease specialist was asked for as a part of an institutional antimicrobial stewardship program.

Setting and method: A prospective study, conducted in a secondary hospital, from 1.7.2017 until 31.7.2017. Demographic data, medical status, laboratory findings and medication lists were collected from eligible patients' files. QT interval was measured by a pharmacist. QTc correction was made using two different equations (Bazzet and Freidricia) and by a VCD adjusted formula when VCD was present. 
Risk of QTc prolongation was calculated using a previously published risk score.

Main outcome measures: Risk of QTc prolongation.

Results: 102 patients were included in the analysis. $49(48 \%)$ females, Median age $=70$ years, average QTc (Bazzet) $=428 \mathrm{~ms}$, average QTc (Freidricia) $=395 \mathrm{~ms}, 21(20 \%)$ patients had VCD, 13 $(12.7 \%)$ were taking one QTc prolonging drug and $36(35.2 \%)$ had 2 or more. $91(89 \%)$ patients were classified as low risk for QTc prolongation, 7 (6.8\%) as intermediate risk, and 4 (3.9\%) as high risk.

Conclusion: Approximately $10 \%$ of the patients had intermediate or high risk for QTc prolongation. Different formulas yielded substantially different corrected QTc intervals. Moreover, $20 \%$ of the patients had VCD which could potentially cause a false diagnosis of prolonged QTc. Pharmacists as experts in medications can be trained to accurately measure and correct the QTc interval and estimate the risk for each patient individually to ensure the safe use of QTc prolonging antimicrobials.

Disclosure of Interest: None Declared.

\section{HP-PC111: New standard of clinical pharmacy service in South Eastern Norway}

E. Trapnes ${ }^{1, *}$, M. N. Davies ${ }^{1}$, S. C. Debes ${ }^{1}$, B. Hedding-Valvik ${ }^{1}$, H. Holm ${ }^{1}$, A. M. Njaastad ${ }^{2}$, A. Johnsen ${ }^{1}$

${ }^{1}$ Hospital Pharmacies Enterprise, South Eastern Norway, ${ }^{2}$ Oslo University Hospital, Oslo, Norway

Background and objective: Hospitals enter into contracts with the hospital pharmacies for clinical pharmacy services. Clinical pharmacists execute not only patient oriented tasks (e.g. medicines reconciliation, medicines review) which have been well described in the Integrated Medicines Management (IMM) model, but also a variety of other tasks like teaching, clinical governance, drug stock holding, developing procedures and advising on drug related matters are regularly carried out. Clinical pharmacy services are slowly increasing in our region, and a definition and description of the service is warranted.

Design: Project design. The steering group consisted of leaders from the hospital pharmacies and hospitals within the region. The project group mainly consisted of clinical pharmacists, a pharmacy director and a consultant. The main goal of the project was to define the clinical service, its content and quality. Project decisions were mainly based on scientific publications, clinical pharmacists' experiences from various ward units and the ward units' needs.

Results: Our clinical pharmacy service's definition: Pharmacists in close collaboration with other healthcare professionals in hospital contribute to increased patient safety by optimizing drug use and drug handling for the individual patient from admission to discharge. Patient oriented tasks, as well as 'ward related tasks' make up the service. The 'ward related tasks' main objectives are to contribute to reasonable health economy and increase competency on medicines management and drug handling in the ward unit. The clinical pharmacist should spend most time executing patient oriented tasks.

Each task has been described, and the description includes purpose, content, time for deliverance and by whom the task is delivered. Steps considered crucial to service quality have been highlighted. A general estimate of time has been specified.

A general recommendation on how a service should be initiated, which tasks the pharmacist should prioritize and which patients to prioritize has been made.

Conclusion: The service of clinical pharmacy has been described in a new standard of clinical pharmacy. Our future recommendation on clinical pharmacy services delivered to hospital ward units will be based on this standard. We anticipate that the service description will make the hospital wards more aware of the possible use of pharmacist resources.

Disclosure of Interest: None Declared.

HP-PC113: Pharmacist on hospital ward-implementation of one of the first clinical pharmacy services in Poland

K. B. Urbańczyk ${ }^{1, *}$, W. Z. Iwanowski ${ }^{1}$, R. Grendziak ${ }^{1}$, W. Witkiewicz ${ }^{1}$

${ }^{1}$ The Regional Specialist Hospital with Reaserch and Develompment Center, Wrocław, Poland

Background and objective: Study covering 6-months period assessed one of the first clinical pharmacy services implemented in Poland to determine possible role for pharmacist on hospital ward.

Setting and method: One pharmacist enrolled in postgraduate course in clinical pharmacy was placed on vascular surgery ward. Routine activities included participation in medical rounds, obtaining and reviewing patient's medication profiles together with daily review of laboratory results, advising patients and medical staff on correct use of medicines. Drug-related recommendations were made where necessary.

Main outcome measures: All interventions were recorded daily and placed in database according to following categories: types of recommendations, stage of patient's hospitalization on which it occurred, therapeutic class of involved medicines. Frequency and acceptance rate of suggestions was monitored during the process.

Results: Pharmacist performed 501 interventions over study period, among which $98 \%$ were accepted by physicians. Over $70 \%$ of them occurred while monitoring patient's during hospital stay and the rest either on admission or discharge. The most frequent type of intervention was drug discontinuation, followed by addition of new or missing drug and change of administration route. The four drug classes most often associated with given recommendations were alimentary tract and metabolism, systemic anti-infectives, nervous system and antithrombotic agents.

Conclusion: The clinical pharmacist can successfully become a member of ward team by making a large number of suggestions in relation to pharmacotherapy which are well perceived by physicians. Involvement can be visible at all stages of patient's hospital stay. Disclosure of Interest: None Declared.

\section{HP-PC114: Analysis of HIV patients admitted to hospital}

\section{A. M. Valle Díaz ${ }^{1, *}$, R. Álvarez ${ }^{2}$, R. García ${ }^{1}$}

${ }^{1}$ Servicio de Farmacia, Hospital Virgen de Las Nieves, ${ }^{2}$ Servicio de Farmacia, Hospital Campus de La Salud, Granada, Spain

Background and objective: HIV patients constitute a group of patients to whom strict control of their pharmacotherapy must be carried out. These are complex pharmacotherapeutic schemes, with risk of interactions and in which adherence to treatment is essential. A hospital admission can cause imbalances that affect the immune status of the patient, even forcing to modify its anti-retroviral therapy (ART). The aim of this study is to study the main characteristics of HIV patients admitted to the hospital, analyzing several parameters: reason for admission, virological and immunological status at admission, ART used during admission, possible drug interactions and adherence to treatment before and after admission.

Design: All patients diagnosed with HIV infection and admitted to the hospital during the period August 2017-December 2017 were selected. For each of them was checked: the medical history, the medical prescription during the admission and the dispensation records of the computer program of outpatient pharmaceutical care. 
Adherence to treatment was calculated in the 3 months before and after admission. Finally, a scientific literature search was performed to identify potential drug interactions.

Results: A total of 48 patients were analyzed. The average age was 49 years. $56 \%$ of the patients had acquired HIV infection due to being addicted to drugs by parenteral route. Regarding the virological situation, the majority had an undetectable viral load (46\%), but also a significant percentage (34\%) had a viral load of more than 10,000 copies. $29 \%$ had CD4 lymphocyte values below 200 cells $/ \mathrm{mm}^{3}, 43 \%$ between 200 and 500 cells $/ \mathrm{mm}^{3}$ and the $28 \%$ of patients had values over 500 cells $/ \mathrm{mm}^{3}$. The causes of admission were very varied, highlighting cardiovascular $(25 \%)$ and respiratory $(14 \%)$. The ART was modified to $20 \%$ of the patients during the admission, mainly due to inefficiency and the appearance of resistances. In 5 cases, the patient did not take any antiretroviral treatment and was instituted at the time of admission. Patients had an average adherence before admission of $94 \%$. However, after admission, the adherence of all patients was lower. Even in 7 patients the adherence dropped more than $10 \%$. Regarding drug interactions, 18 relevant clinically interactions were found. The most common were associations of protease inhibitors with benzodiazepines (12 patients). In 3 patients were detected combinations of drugs not recommended in clinical practice guidelines because of increased risk of Q-T interval. This was the case of darunavir/salmeterol association.

Conclusion: The hospital admission of HIV patients is mostly related to the poor virological and immunological status of the patient. Adherence is affected in some cases, which leads to an important adherence control after admission to this type of patients. The incidence of adverse effects is also important, greater attention from the pharmacist is needed. It is necessary to pay attention to concomitant medication in order to detect interactions and assess the risk-benefit ratio of determined combinations.

Disclosure of Interest: None Declared.

HP-CE003: A feasibility study for the identification of patients with iatrogenic risk eligible for a reinforced discharge conciliation using a specific pharmaceutical interview in an infectious disease department

\section{J. Baba ${ }^{1, *}$, L. Surgers ${ }^{2}$, P. Hindlet ${ }^{1}$, C. Fernandez ${ }^{1}$, A. Daguenel-Nguyen ${ }^{1}$}

${ }^{1}$ Pharmacy Department, ${ }^{2}$ Infectious and Tropical Diseases Department, University Hospitals East Paris Huep - Saint-Antoine Site, Public Assistance-Paris Hospitals (AP-HP), Paris, France

Background and objective: In our hospital, the entrance medication conciliation has been introduced within the infectious diseases department for several years but the discharge conciliation is being evaluated for its feasibility. The reinforced discharge conciliation must be accompanied by specific information for the patient according to the HAS in order to secure their discharge.

Main objective: Identify at-risk patients for whom an improved discharge conciliation is needed and provide them with a specific information via a pharmaceutical interview.

Design: Prospective study carried out in an Infectious Disease department for 40 days.

Patients were selected during the entrance conciliation according to accepted iatrogenic risk criteria: age $>75$ years, polypharmacy, socio-economic situation, cognitive disorders, risk of nonobservance, high-risk drugs in progress $(\mathrm{VKA}, \ldots)$, usual treatment change, continuation after anti-infection treatment, or according to medical opinion.

Criteria for non-eligibility: patients benefiting from carers for the medication taken out of hospital, hospitalization less than 3 working days.
During the outpatient conciliation, eligible patients had an interview with the clinical pharmacist and benefited from specific documentation: good use sheets, information for special status medicines, treatment plan, hospital/city liaison sheets.

Results: 204 hospitalized patients from March 14 to April 30, 2018. 168 patients discharged from hospital. 153 patients scored. 50 (33\%) eligible for a reinforced conciliation. 28 pharmaceutical interventions. 3 had a therapeutic education plan, 7 patients came out with sheets for good use, 6 patients with a liaison sheet, 3 with a treatment plan. $33 \%$ of scored patients were eligible for an improved conciliation with a pharmaceutical interview but $45 \%$ of these patients actually benefited.22 patients could not benefit from improved conciliation due to: unavailability of the intern in pharmacy, late prescription in the department.

Conclusion: These results could validate the feasibility study that identified the patients at risk for whom an improved discharge conciliation is necessary.

Patients at risk were able to receive specific information using appropriate tools.

The process of preparing the enhanced discharge conciliation requires a preparation time that must be taken into account in the operation of the department.

A study on a larger scale could validate an eligibility score for these at-risk patients.

Disclosure of Interest: None Declared.

HP-CE004: Mentoring for Foundation Programme: what do our mentees expect of their mentors?

J. Brown ${ }^{1, *}$

${ }^{1}$ Pharmacy and Medicines Management, Antrim Area Hospital, Northern Health and Social Care Trust, Antrim, United Kingdom

Background and objective: Junior pharmacists may undertake training in the Foundation Programme Certificate provided by Northern Ireland Centre for Pharmacy Learning and Development. This course covers competency based learning in dispensary, technical services, evidence based practice and medicines management. An Educational Supervisor (mentor) is allocated to assist them through their programme, ensuring that the competencies are matched by appropriate evidence and signing their portfolio for submission and assessment. Mentoring has many benefits for the mentee as well as the mentor. In this study the mentee perception of the mentoring process, their previous experience being mentored and their expectations of a good mentor are examined.

Design: All Foundation Programme participants were surveyed in 2014 and then annually thereafter to determine their views on the qualities a good mentor should have, their previous experiences and the advantages and disadvantages of being mentored. An 8-item questionnaire was developed, with the majority of questions being free text, and distributed once to each participant when they started the programme. The response rate was $49 \%$. Ethics approval was not required.

Results: Although this was a small study capturing the views of a limited number of possible respondents, the information gleaned has proved very beneficial in the development of mentor support and also managing mentee expectations. When asked what the main qualities that participants felt mentors required, $90 \%$ of respondents answered being approachable, followed by knowledgeable (50\% of respondents), supportive (50\%), experienced (40\%), enthusiastic (40\%), motivating $(40 \%)$, organised $(35 \%)$ and providing honest feedback (30\%). In their previous experience of mentoring, 16 of the 20 respondents reported that having a mentor was a positive experience and one stated that this had been a negative experience as they were working and felt like they weren't mentored. Although having a 
mentor is a pre-requisite for entry to Foundation Programme, the vast majority of participants recognise that there are a number of advantages to having a mentor. For example; "having a mentor helps you to work towards meetings and reviews", "my mentor had already been through the programme and understands what is required to successfully complete it". Other statements showed a holistic and deeper supportive relationship. For example; "someone to talk to when you are worried or don't know something" and "provides valuable feedback and suggestions that enable you to improve". One respondent stated that having a mentor who had not completed the course may be a disadvantage as they may not know the portfolio requirements.

Conclusion: Mentoring junior pharmacists can be an extremely rewarding relationship for both mentor and mentee. The mentee views gained from this study has helped developed mentor training and recruitment by helping mentors understand the qualities the mentees require and the expectations of their mentee. It is clear that mentees value the mentoring relationship, not only in the completion of academic work but also in their professional and personal development as a clinical pharmacist.

Disclosure of Interest: None Declared.

\section{HP-CE005: 'Best foot forward': an analysis of a pharmacology update for podiatrists}

\section{J. Brown ${ }^{1, *}$, J. Cundell ${ }^{2}$, P. McMullan ${ }^{3}$, A. Campbell-Smyth ${ }^{4}$}

${ }^{1}$ Pharmacy and Medicines Management, Northern Health and Social Care Trust, Antrim, ${ }^{2}$ School of Health Science, Ulster University, Newtownabbey, ${ }^{3}$ HSC Clinical Education Centre, Belfast, ${ }^{4}$ Podiatry Department, Northern Health and Social Care Trust, Ballymena, United Kingdom

Background and objective: A 2-day training pharmacology update for podiatrists was designed and delivered by podiatrists and clinical pharmacists in December 2017 in collaboration with the HSC Clinical Education Centre who facilitated the programme on behalf of the Department of Health, NI. The clinical impact of the course and quality of teaching was analysed.

Design: The purpose of the pharmacology education and training was to provide information on prescription only medicines, medico-legal implications of independent prescribing, pharmacology of common drugs and the potential for adverse drug reactions. This was delivered by a number of podiatrists and clinical pharmacists in 40 min sessions. Clinical topics included pharmacokinetics and pharmacodynamics, rheumatology, renal, oncology, diabetes, vascular, neurological disorders, pain and antibiotics. Other sessions included: legislation, independent prescribing and the importance of a good drug history. The course ended with an interactive workshop which focused on adverse reactions, interactions, consideration of patient factors, compliance and issues with medication histories. Within 5 days of the course finishing the course participants were asked to complete an on-line questionnaire.

Results: Sixty of the 67 (90\%) participants completed the on-line questionnaire with $97 \%$ stating that that the course met its objectives and $92 \%$ were satisfied or very satisfied with the delivery of the programme. The most beneficial areas covered were clinical update (28\%), general update (18\%) and the interactive workshop (17\%). When asked how respondents intended to use their learning the most rated areas were; $30 \%$ would improve the quality of services or patient care, 28\% for Continued Professional Development (with 53\% identifying further learning needs following the course), $25 \%$ stated a great awareness of medicines and $15 \%$ would review patient medication histories. For the overall assessment of the programme 95\% stated that the course was good, very good or excellent and $95 \%$ would recommend this programme.
Conclusion: The pharmacology update for podiatrists course proved very valuable and participants were satisfied with the delivery of the programme. Attendees stated that they would aim to improve patient care through improved knowledge of common medicines and patient advice, interactions and confidence to review patients' medicine and medication histories. The delivery of this workshop by pharmacists and podiatrists in collaboration with the HSC Clinical Education Centre has also increased knowledge of the role of the multi-disciplinary team and highlighted common pharmaceutical care issues that podiatrists may encounter in their clinical practice.

Disclosure of Interest: None Declared.

HP-CE006: An error room in an outpatient pharmacy unit: tool to improve practice

C. Christen ${ }^{1, *}$, T. Collignon ${ }^{1}$, A. Berthe-Aucejo ${ }^{1}$, S. Leylavergne ${ }^{1}$, N. Meddour ${ }^{1}$, N. Brassier ${ }^{1}$, F. Huet ${ }^{1}$

${ }^{1}$ Service Approvisionnement et Distribution, Agence Générale des équipements et produits de santé, Nanterre Cedex, France

Background and objective: The safety of patient care is essential and could be improved by health simulation tools. The error room could help to educate professionals in a playful way. The outpatient hospital pharmacy is a pharmaceutical unit which provides treatments to 2178 patients throughout France, mostly affected by hereditary metabolic diseases. One critical step was the prescription's input control, which took place after the input of prescriptions before treatment preparation. The objective was to implement an error room regarding this step for pharmacy dispensers (PD).

Design: Errors were selected on adverse events' database, and from previous risk analysis. Six fake patient files including 16 errors were created, with a maximum of 5 errors by file. Five classes of errors were concerned: dispensing, traceability, pharmaceutical analysis, pharmaceutical regulation, and identity vigilance. Files were approved and tested by the unit's pharmacists (UP) and health executives (HE). During a briefing before simulation, the objective of error room and instructions were explained to PD. They entered in the room to analyze fake files according to a planning. The training session was not limited in time. At the end, a debriefing with UP and HE was done to review each error reminding them to the good practices. PD satisfaction was evaluated by a survey.

Results: All PD working in the unit $(\mathrm{PD}=7)$ participated to the error room. The median time of passage was $47 \mathrm{~min} \quad(\mathrm{Q} 1=42.5$ $\mathrm{Q} 3=50 \mathrm{~min})$. The median error detection rate was $87.5 \%(\mathrm{~min}=62-$ $\max =100 \%$ ). Pharmaceutical analyzes errors were systematically recorded, while traceability errors were detected in $62 \%$ of cases. Rates of identity vigilance error, pharmaceutical regulation and dispensing errors were respectively $72 \%$ (10/14), 91\% (19/21), 95\% of cases (38/40). As a result, $86 \%$ of PD was satisfied. The median mark for the tool was 8.6/10. The workshop met PD's expectations and they acquired useful knowledge.

Conclusion: The error room in a pharmaceutical unit has been successfully implemented and allowed to improve PD's knowledges and practices. Procedures and documents will be updated and training about identity vigilance will be planified. Another error room on the preparation of treatment will be created.

Disclosure of Interest: None Declared.

HP-CE007: Evaluating user satisfaction with an electronic prescription system

M. Martín Marqués ${ }^{1}$, A. De Dios Lopez ${ }^{1}$, E. Parada Ricart ${ }^{2}$, P. A. López Broseta ${ }^{1}$, J. del Estal Jiménez,"*, M. Canela Subirada ${ }^{1}$, L. Canadell Vilarrasa ${ }^{1}$ 
${ }^{1}$ Pharmacy, ${ }^{2}$ Paediatrics Service, Hospital Universitari Joan XXIII de Tarragona, Tarragona, Spain

Background and objective: Electronic prescription (EP) has been proposed as an important strategy to reduce medication errors, improve the quality of patient care and create savings in health care costs. Despite these potential advantages, user satisfaction plays a significant role in the success of its implementation.

The purpose of this research was to evaluate satisfaction surveys on the implementation of EP in Paediatrics Service.

Design: We made an anonymous survey to nurses and doctors before and after the EP implementation in Paediatrics Service. We made a bibliographic research and we chosed a validated survey and we have adapted it.

The previous implementation survey included 3 questions about the disadvantages, the advantages and if the responder considers the EP as a better option than paper prescriptions. They are multiple choice questions.

The post implementation survey included 5 questions about the disadvantages, the advantages, the satisfaction level with: the formative sessions and with the follow-up made by the Pharmacist and the satisfaction level with the EP. The last 3 questions have a 1-10 punctuation and the other questions are multiple choice.

Results: 25 health professionals were surveyed, 22(88\%) were women. The average age was: $39 \pm 12$ years old. $12(48 \%)$ were doctors and $13(52 \%)$ were nurses.

In the previous implementation survey, the most reported disadvantages were: 19(76\%) the failure of computer network and 13(52\%) the difficulty of program $\left(\right.$ Silicon $\left.{ }^{\circledR}\right)$ management. And the most reported advantages were: $22(85 \%)$ actualised treatment availability, $22(85 \%)$ less hand-writing interpretation errors and $22(85 \%)$ less transcription errors. $18(72 \%)$ professionals considered EP as a better option.

In the post implementation survey, the most reported disadvantages were: $15(60 \%)$ the lack of computers and $9(36 \%)$ the failure of computer network. The most reported advantages were: $22(85 \%)$ actualised treatment availability, 22(85\%) less hand-writing interpretation errors. The scores were: $8.5( \pm 1.9)$ for the satisfaction level, $9( \pm 1.5)$ for the Pharmacist's follow-up and $8.9( \pm 1)$ for the satisfaction level with the EP.

Conclusion: The concers before the EP were confirmed after the implantation.

- Nurses and doctors are satisfied with the EP implantation and with the pharmacist assistance at the beginning and during the follow up.

Disclosure of Interest: None Declared.

HP-CE008: Individual pharmacist-led consultation dedicated to outpatients introducing a Compassionate Use Program by a hospital ambulatory pharmacy: a prospective pilot study

\section{Diet ${ }^{1, *}$, V. Nave ${ }^{1}$, S. Garcia ${ }^{1}$, I. Carpentier ${ }^{1}$, K. Beny ${ }^{1}$}

${ }^{1}$ Pharmacie centrale, Hospices Civils de Lyon, Lyon, France

Background and objective: Patient's access to pharmaceutical information is usually limited for treatment in Compassionate Use Programs, called "nominative Temporary Authorization for Use" (ATUn) in France. Limited information can increase the risk of adverse drug effects (unknown side effects, drug-drug interactions...), particularly for outpatient. Therefore, pharmaceutical consultations have been set up in a hospital ambulatory pharmacy, targeting patients that start a drug through ATUn program. These consultations are provided during the first two drug dispensing. The first consultation aims at giving information about the treatment, through document given to the patient. The second consultation aims to check the way patient understood information, his compliance and the tolerance of the treatment. The purpose of this study is to evaluate the interest of this pharmaceutical consultation and give a first feedback.

Design: A prospective pilot study was carried out from November 2017 to April 2018. All ambulatory patients who initiated a treatment on ATUn program in our unit were enrolled. Evaluation criteria were: patient knowledge improvement (a binary score is assigned to 6 items at every consultation), patient compliance (Morisky scale (1)), patient satisfaction (satisfaction survey).

Results: On the 12 patients that initiated a treatment on ATUn program, 7 patients were enrolled. Among them, 4 patients benefited of two consultations. All patients had poor basic knowledge about their new medication (evaluating score $=0$ or 1 ). Patient knowledge about their treatment improved by 3 or more points between the two consultations (the mean value of second evaluating score was 4). One pharmaceutical intervention was realized and accepted by prescriber. Furthermore, a physician was contacted to inform him about a possible pharmacokinetic drug interaction. Compliance was good for all patients (Morisky scale $\geq 6$ ). All patients reported a high satisfaction about the given document and $80 \%$ were satisfied with the answers delivered by pharmacist to their questions. All patients evaluated the consultations usefull.

Conclusion: This pilot study confirms the interest of pharmacist-led consultations for individual care, particularly for outpatients with drugs on the ATU program. The main limitations of the study are the low number of enrolled patients and the lack of comparator group of a non-comparative study. We aim at developing these consultations in the other outpatient dispensing units in our hospital, in order to increase the number of enrolled patient and evaluate the long term impact.

\section{Reference}

1. Morisky DE, Green LW, Levine DM. Concurrent and predictive validity of a self-reported measure of medication adherence. Med. Care. 1986;24:67-74.

Disclosure of Interest: None Declared.

HP-CE011: Changes in patient safety during medication preparation and administration: experiences from the implementation of a Closed Loop Medication System

A. Mulac ${ }^{1, *}$, R. Kristiansen ${ }^{2}$, H. Chairi ${ }^{1}$, M. Bayar ${ }^{1}$, A. G. Granas ${ }^{1}$

${ }^{1}$ School of Pharmacy, University of Oslo, Oslo, ${ }^{2}$ Ostfold Hospital Trust, Kalnes, Norway

Background and objective: Closed loop medication system (CLMS), using scanning of barcodes on medicines and patients' wristbands, can reduce medication errors. Hospitals in Norway are still in an early stage for utilizing this technology. The aim of this study was to evaluate nurses' experience and reflections of how CLMS affects patient safety during medication preparation and administration.

Setting and method: Semi-structured focus group interviews in a hospital prior and after the implementation of a.

CLMS pilot in 2017. Prior to implementation, focus group themes were: Errors and omissions, medication procedures in the medicine room, the ward and in the patient rooms. After implementation, focus group themes were: Errors and omissions, experiences with CLMS and new technology, use of professional knowledge with new medication procedures, and how CLMS affected work-flow. The data was analysed thematically by using systematic text condensation. 
Main outcome measures: Experiences with using the CLMS during preparation and administration of medicines.

Results: Two focus groups were held in March 2017 and January 2018 with a total of 13 nurses from four different hospital wards. Positive aspects of CLMS was confirmation of correct medicines to the correct patient and safer medication procedures. The nurses however reported that it was more time-consuming to use the CLMS than a manual system. The actual design of the medicines trollies were not optimal: they had too little space for the lap-tops used during scanning, and were cumbersome to manoeuvre in the patient rooms. Some also use the trolly surface top for intravenious preparation, making CLMS procedures diffcult to follow.

Conclusion: The nurses said the main barriers were to adapt to the new CLMS work-flow and that it was more time consuming. The nurses however agree that CLMS promotes standardization of medication preparation and administration routines, and potentially improves patient safety.

Disclosure of Interest: None Declared.

HP-CE012: Objective Structured Clinical Examination (OSCE): a new exam for a German pharmacy course

\section{R. Mathis ${ }^{1,2, *}$, C. Culmsee ${ }^{1}$, R. Radziwill ${ }^{2}$, A. Freidank ${ }^{2}$}

${ }^{1}$ Philipps-University Marburg, Marburg, ${ }^{2}$ Klinikum Fulda gAG, Fulda, Germany

Background and objective: Today's demands on pharmacists to participate in patients' therapy as part of a multi-professional team should already be considered in university courses. Internationally, OSCEs proved their role as an assessment method, especially in terms of medicine, nursing and pharmacy courses.

The aim of OSCEs is to assess the students' professional performance in a simulated, controlled environment. Besides the students' clinicalpharmaceutical knowledge, further competencies such as problemsolving, communication and counselling skills are evaluated.

To evaluate the reliability, validity and feasibility of OSCEs to provide a scientific basis for implementation of OSCEs in pharmacy courses in Germany.

Setting and method: In summer semester 201724 pharmacy students of Philipps-University Marburg attending the 8th semester and 20 students of the 7 th semester were being assessed in a 12-stationOSCE, including 6 theoretical and 6 practical stations. In winter semester $2017 / 189$ of those 20 students were tested again with a 12-station-OSCE, another 19 OSCE-naive students in a 10-stationOSCE. Participation was voluntary. In July 2018 a mandatory OSCE will be conducted for about 80 students attending the 8 th semester of Philipps-University Marburg.

Main outcome measures: OSCE reliability is assessed using Cronbach's alpha, validity by means of correlation with the final examination and grades achieved in state examination. OSCEs were evaluated by the participants.

Results: In summer semester 2017 Cronbach's alpha for 8th semester-OSCE is 0.696 , for 7 th semester-OSCE 0.589 and in winter semester $2017 / 180.756$, indicating a moderate reliability. There is no correlation between the OSCE, final exam and state examination. The students would appreciate OSCEs as part of their curriculum.

Statistical data for the OSCE in summer semester 2018 will be available in September 2018.

Conclusion: OSCEs are a reliable and feasible, while time- and personnel-intense assessment method that students in particular ask for to be included in the Pharmacy curriculum in Germany. For further investigation, especially on correlation, July's OSCE data with a larger cohort are awaited.

Disclosure of Interest: None Declared.
HP-CE014: Knowledge and attitudes of pharmacovigilance and ADRs spontaneous reporting among pediatricians and paediatric residents in Jordan

\section{T. L. Mukattash ${ }^{1, *}$, M. Alwadi ${ }^{1}$}

${ }^{1}$ Department of Clinical Pharmacy, Jordan University of Science and Technology, Irbid, Jordan

Background and objective: Pharmacovigilance is the practice of monitoring the effects of medical drugs after they have been licensed for use. The use of medicines in children has always carried its own set of risks when compared to the use of medicines in adults. It is well documented in the literature that paediatrics bare three times the chance of suffering from adverse drug reactions (ADRs) compared to the adult population. Spotting ADRs and reporting them will lead to more safe medicine use in paediatrics.

The present study aims to assess paediatricians and paediatric residents' knowledge, attitudes, and practices of pharmacovigilance which could be a progressive step towards enhancing their participation in spontaneous ADRs reporting.

Setting and method: This is a cross-sectional study that was conducted in 2017 where 200 paediatricians and paediatric residents from different hospitals in Jordan were approached and asked to fill and complete a validated structured questionnaire.

The structured questionnaire used in this study was adopted from a study by Suyagh et al. The questionnaire consisted of four sections, (1) socio-demographic characteristics, (2) knowledge of respondents about pharmacovigilance, (3) attitudes of respondents towards pharmacovigilance, and (4) respondents' practices of pharmacovigilance. Main outcome measures: Knowledge and attitude scores where calculated based on respondents answers to part two and three of the questionnaire, those where categorized as good, average, and low.

Results: A total of 200 respondents were approached to take part in this study, of those 142 agreed to participate $(71 \%)$. The majority of respondents were male $(72.7 \%)$ and almost one-third of respondents were specialists $(36.5 \%)$.

Only $25.2 \%$ respondents have heard of pharmacovigilance before this study. Of the respondents $21.3 \%$ have indicated that they heard of pharmacovigilance during their undergraduate or postgraduate studies, only $27.3 \%$ have correctly defined pharmacovigilance and almost one-third of respondents (32.1\%) were able to state the main purpose of pharmacovigilance. Only $3.6 \%$ of respondents were aware of the existence of a national pharmacovigilance center in Jordan and $11.5 \%$ of respondents knew about the yellow card system while $75 \%$ were not aware of the means of obtaining it. A minority $(6.3 \%)$ knew that health-care-professionals and patients are responsible for ADRs reporting. Almost $87 \%$ of respondents were classified to have a poor knowledge score, whereas $12 \%$ and $1.4 \%$ of respondents had an average and good score, respectively.

Almost $59 \%$ of respondents had a good attitude score, whereas $24.6 \%$ and $16.9 \%$ of participants had average and poor scores, respectively.

Conclusion: The results of the present study expose the fact that pediatricians lack knowledge of PV and ADRs reporting. However, they have a good attitude towards ADRs reporting and enhancing their PV practice. This is still not reflected in their own practice. Further training and education about ADRs reporting is very important to move toward safer medications in children.

Disclosure of Interest: None Declared.

HP-CE016: A model of medication reconciliation module integrated into the electronic patient record

T. Rodier ${ }^{1, *}$, A. Kerrad ${ }^{1}$, N. Curatolo ${ }^{2}$, T. Tritz ${ }^{3}$, A. Gohari ${ }^{1}$, I. Debrix ${ }^{1}$, F. Federspiel ${ }^{1}$ 
${ }^{1}$ Pharmacy Department, Hôpital Tenon-APHP, PARIS, ${ }^{2}$ Pharmacy Department, Hôpital Antoine Béclère-APHP, Clamart, ${ }^{3}$ Pharmacy Department, Hôpital Ambroise Paré-APHP, Boulogne-Billancourt, France

Background and objective: In the context of the development of an electronic pharmacy record as a part of the electronic patient record, we propose a model of electronic medication reconciliation module connected with prescribing software.

Design: An on-line survey was addressed to an expert panel of hospital pharmacists in order to define their expectations about the structure and the features of the module. Then, a model based on the expressed needs was built. Lastly, technical requirements were assessed by the information technology department in charge of the pharmacy record development.

Results: A three-parts model including each step of the medication reconciliation process on admission was designed. The first part comes as a data-collecting table including for each line of drug: name, dosage and frequency of administration. Data are added thanks to an autocomplete system, based on both hospital and national drug formularies on the model and wording of the prescribing search field. Each source is extensively detailed, allowing conflicting informations to be recorded as well. Lastly, a specific column is provided for comments about self-medication or compliance items. The user finally selects all the wanted data to be sent to the next part.

The second part is the Best Possible Medication History (BPMH), automatically generated from the data-collecting table. A validation button allows the BPMH to be printed or directly inserted into the electronic medical record.

The third part comes as a dynamic table that imports the hospital prescription directly from the prescribing software, to be compared with the BPMH, in order easily to identify potential discrepancies.

Another part of the module enables to record a lot of informations about the patient, by manual input or by extraction from the medical record. PDF files can also be created and attached to save paper prescriptions.

Conclusion: The model we propose here as a reconciliation medication electronic module may represent a valuable tool to improve efficiency and security of prescribing. Designed as part of the medical record, this module offers time saving in data integration, limited transcription errors, readability and accessibility of the BPMH for clinicians, in proactive as well as in retroactive processes. Furthermore, an important step is taken in terms of traceability and dematerialization of the process: with no more paper or archives, it records all explored sources, including conflicting ones, and the act of signature of the BPMH is promoted, becoming mandatory for further export or edition. Lastly, the autocomplete system for drug integration based on a common drug formulary seems to be the best expected progress, facilitating and securizing steps of data input and drug comparison. We plan now to think about an extended feature allowing pharmacists to prepare the admission prescription directly from the BPMH.

Disclosure of Interest: None Declared.

HP-CE017: Evaluation of appropriate use of low molecular weight heparin prescriptions in a French hospital

\section{T. Vallecillo ${ }^{1, *}$, E. Michelet Huot ${ }^{1}$, C. Mongaret $^{1,2}$, D. Hettler ${ }^{1}$}

${ }^{1}$ Department of Pharmacy, ${ }^{2}$ Faculty of Pharmacy, CHU Reims, Reims, France

Background and objective: Low molecular weight heparins (LMWH) are prescribed for prophylactic and therapeutic indications and are involved in many drug related problems. In 2017, to be in line with the European guidelines, the French Summaries of Product Characteristics (SmPC) was modified for enoxaparin dosage in several indications and its use in severe renal failure. The objective of this study is to assess the appropriate use of LMWH in our hospital, according to the SmPC and bibliographic review.

Design: Observational study during 1 week all patients treated with LMWH (enoxaparin sodium and tinzaparin sodium, LMWH referenced in ourhospital) in 9 medical wards (computerized medical wards), none being a surgical service. Evaluation of the appropriate dosage for all LMWH prescriptions (indication and renal function) according to the updated SmPC and bibliographic review. A LMWH prescription is described as inappropriate if it use was not appropriate according to SmPC and bibliographic review. Data collection: on patient (weight, body mass index, renal function according to CKD EPI, antiXa activity) and LMWH medication (prescribed molecule, indication, dosage).

Results: A total of 178 patients were treated with LMWH, 172 were included (no weight for 6 patients). The average age was 63 [14-99] years. The average weight was 71.1 [36.7-130.0] kg. Twenty-eight patients $(15.9 \%)$ received LMWH for curative indications: $7(25.0 \%)$ with tinzaparin sodium and $21(75 \%)$ with enoxaparin sodium. All tinzaparin prescriptions $(100 \%)$ were prescribed according to appropriate use. For the enoxaparin prescriptions, 6 (28.6\%) were described as inappropriate due to a wrong weight-adjusted dosing, 4 (19.0\%) due to a non-adjustmentof the enoxaparin dose accordinglyof antiXa activity and $1(4.8 \%)$ due to $100 \mathrm{IU} / \mathrm{kg}$ daily Two prescriptions were not in accordance with the updated SmPC should have received $150 \mathrm{UI} / \mathrm{kg}$ daily.

Of the 144 patients received LMWH for prophylactic indications, all were receiving enoxaparin sodium. There were a total of $105(72.9 \%)$ appropriate and 39 (27.1\%) inappropriate prescriptions. Five patients had 2 problems per prescription, for a total of 44 drug related problems. These inappropriate prescriptions were related to dosage problems: according to weight $(89.7 \% ; 36)$, according to indication (10.3; 4), according to renal function $(10.3 ; 4)$.

Conclusion: Twenty-two prescriptions were described as inappropriate, only the enoxaparin prescriptions. The weight-adjusted dosing was not appropriate according to SmPC and bibliographic review for weight $>100 \mathrm{~kg}$ or $<50 \mathrm{~kg}$.

Despite the release of information at the time of the new enoxaparin sodium European guidelines, we note that more targeted information on dosage adjustment by weight would be required for prescribers.

Tinzaparin is largely unused on our establishment, because it is only referenced for curative doses; our sample is too small to be able to evaluate the real conformity of these prescriptions.

Disclosure of Interest: None Declared.

TDMP001: Pharmacokinetics of Ertapenem in obese patient with osteitis to Escherishia Coli ESBL

C. Chabut $^{1, *}$, P. Tailpied ${ }^{2}$, M. Garnier ${ }^{2}$, F. Busato ${ }^{2}$, J. Giraud ${ }^{3}$, T. Dulac ${ }^{2}$, P. Petua ${ }^{2}$, L. Massias', A. Le Coustumier ${ }^{4}$, M. P. Le ${ }^{1}$

${ }^{1}$ 75018, APHP, Hôpital Bichat-Claude Bernard, Laboratoire de Pharmaco-Toxicologie, Paris, ${ }^{2} 65000$, Centre Hospitalier de Tarbes, Service de Réanimation Polyvalente, Tarbes, ${ }^{3} 75018$, APHP, Hôpital Bichat-Claude Bernard, Service de Pharmacie, ${ }^{4} 75000$, IAME, UMR 1137, Université Paris Diderot, Sorbonne Paris Cité and INSERM, Paris, France

Background and objective: Ertapenem is actually a broad spectrum antibiotic used in last resort into skin and skin structure infections. A 71 -years-old obese patient $\left(105 \mathrm{~kg}\right.$, BMI $\left.41 \mathrm{~kg} / \mathrm{m}^{2}\right)$ with comorbidities attended the emergency ward for multiple sternal and ribs fractures. 
A sacral pressure ulcer was reported with an osteitis as probable mode of entry and Escherichia coli producing ESBL was found in blood cultures and urines.

The objectives were to assess the pharmacokinetics parameters of ertapenem and its penetration in bone in an obese patient.

Setting and method: Ertapenem was administred twice-daily in slow intravenous infusion of $2 \mathrm{~g}$ over $30 \mathrm{~min}$, dual-therapy with fosfomycin (4 $\mathrm{g}$ four times daily). Blood samples were collected every hour during $24 \mathrm{~h}$ and antibiotic concentrations assayed by HPLC-UV. PK parameters were calculated by non-compartmental analysis ( $\mathrm{R}$ version 3.3.2, https://cran.r-project.org/). Ertapenem $\mathrm{AUC}_{0-12}$ and fosfomycin $\mathrm{AUC}_{0-6}$ were determined using composite trapezoid rule. Main outcome measures: To evaluate total $\left(\mathrm{plasma}_{\text {total }}\right)$ and unbound (plasma ${ }_{\text {unbound }}$ ) plasma ertapenem and plasma $a_{\text {total }}$ fosfomycin and their minimum inhibitory concentration (MIC).

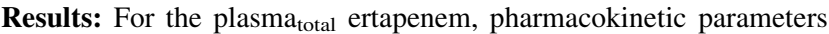
were: clearance CL $5.25 \mathrm{~L} / \mathrm{h} / \mathrm{kg}$, volume of distribution V $0.43 \mathrm{~L} / \mathrm{kg}$, AUC0-12 $389.9 \mathrm{mg} \mathrm{h} / \mathrm{L}, \mathrm{Cmax} 60.9 \mathrm{mg} / \mathrm{L}, \mathrm{Cmin} 15.5 \mathrm{mg} / \mathrm{L}$ and t1/2 6.0 h. For plasma unbound, AUC0-12 $123.9 \mathrm{mg} \mathrm{h} / \mathrm{L}, \mathrm{Cmax} 21.9 \mathrm{mg} / \mathrm{L}$ and $\mathrm{Cmin} 3.5 \mathrm{mg} / \mathrm{L}$. Overall, plasma ${ }_{\text {total }}$ exposure of ertapenem was lower than in normal-weighted patients $(0.12 \mathrm{~L} / \mathrm{kg})$ probably due to

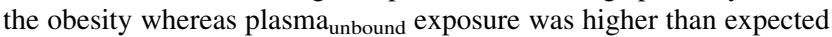
probably due to hypoalbuminaemia and saturable protein binding at high dose.

The efficacy of ertapenem is correlated with the time during which plasma $_{\text {unbound }}$ is $>$ MIC (reported $<0.5 \mathrm{mg} / \mathrm{L}$ in our patient), and had to be greater than $35 \%$ of dosing interval to have a bacterial effect. Conclusion: In summary, our results suggest that ertapenem $(2 \mathrm{~g}$ twice-daily), associated with fosfomycin (4 $\mathrm{g}$ four times daily), might be sufficient to achieve maximum bactericidal ( $f$ T $>$ MIC, $40 \%$ ) activity in obese subjects. The decrease of dosing interval might be an effective solution in order to increase ertapenem plasma exposure in the specific population of obese patients or with a high MIC.

Disclosure of Interest: None Declared.

TDMP002: Retrospective study of gentamicin peak plasma level measured between 2013 and 2017: can we ever achieve the therapeutic range?

A.-F. Dumas ${ }^{1, *}$, H. Pluchart ${ }^{1,2,3}$, M. Bartoli ${ }^{4}$, E. Gautier-Veyret ${ }^{4,5}$, M. Dio ${ }^{1}$, C. Bernardeau ${ }^{1}$, A. Isnard ${ }^{1}$, S. Chanoine ${ }^{1,2}$, P. Bedouch ${ }^{1,2,3}$, O. Epaulard ${ }^{5,6,7}$

${ }^{1}$ Pharmacy Department, Grenoble Alpes University Hospital, ${ }^{2}$ School of pharmacy, ${ }^{3} \mathrm{CNRS}$, TIMC-IMAG UMR5525/ThEMAS, Université Grenoble Alpes, ${ }^{4}$ Laboratory of Pharmacology, Toxicology and Pharmacogenetics, Grenoble Alpes University Hospital, ${ }^{5}$ School of medicine, Université Grenoble Alpes, ${ }^{6}$ Infectious diseases department, Grenoble Alpes University Hospital, ${ }^{7}$ Fédération d'infectiologie multidisciplinaire de l'arc alpin, Université Grenoble Alpes, Grenoble, France

Background and objective: Gentamicin therapeutic drug monitoring is an important parameter to ensure reaching 8-10 times the minimal inhibitory concentration of most resistant bacteria. We aimed to estimate if the recommended dose range for gentamicin $(3-8 \mathrm{mg} / \mathrm{kg}$ daily) leads to sufficient peak plasma level (Cmax) between 30 and $40 \mathrm{mg} / \mathrm{L}$.

Setting and method: A single-center retrospective study was conducted among hospitalized patients receiving gentamicin between January 2013 and September 2017.

Main outcome measures: Gentamicin Cmax, doses, Cockroft and Gault creatinine clearance (Clcr) were collected. Cmax from intensive care units were excluded. Quantitative variables are expressed as median $[\mathrm{q} 1 ; \mathrm{q} 3]$. Spearman correlation test was performed to assess the correlation between quantitative variables.
Results: 140 gentamicin dosages from 73 patients were included. Patients were $65[48 ; 75]$ years old and weighed $68[58 ; 81] \mathrm{kgs}, 91$ $(65 \%)$ of them were men. Renal insufficiency (Clcr $<90 \mathrm{~mL} / \mathrm{min}$ ) was detected among 69 (49.3\%) dosages (serum creatinine: 69 [59; 92] $\mu \mathrm{mol} / \mathrm{L})$. Most patients $(=114(80 \%))$ were hospitalized in medical wards, $28(20 \%)$ in surgical wards. Median gentamicin dosage was $4.6[3.1 ; 5.9] \mathrm{mg} / \mathrm{kg}$, median Cmax was $12.2[8.7 ; 18.6]$ $\mathrm{mg} / \mathrm{L}$. Despite $104(74.3 \%)$ of the doses being in the recommended range between 3 and $8 \mathrm{mg} / \mathrm{kg}$, only $1(0.7 \%)$ of the Cmax reached the therapeutic range (median dosage of $5.5 \mathrm{mg} / \mathrm{kg}$ ). There were 136 (97.2\%) Cmax under $30 \mathrm{mg} / \mathrm{L}$, corresponding to median dosages of $4.5[3.1 ; 5.9] \mathrm{mg} / \mathrm{kg}(102(75 \%)$ Cmax corresponding to the recommended dosage). Cmax superior to $40 \mathrm{mg} / \mathrm{L}$ involved only $3(2.1 \%)$ samples with a dosage equal to $10.0[7.9 ; 14.7] \mathrm{mg} / \mathrm{kg}(1(33.3 \%)$ corresponding to the recommended dosage). Spearman correlation coefficient between Cmax and doses was $0.7(p<0.01)$. Clcr and Cmax appeared not to be correlated.

Conclusion: According to our results, recommended doses do not allow the targeted Cmax to be attained. Although there is a lack of statistical power due to our sample size and the correct interval of blood sampling is uncertain for all dosages, this suggests that higher doses should be used for empiric therapy or that $8-10$ times the minimal inhibitory concentration is an unrealistic aim. To answer this matter, more data are currently being collected.

Disclosure of Interest: None Declared.

TDMP003: Early experience with therapeutic drug monitoring of vedolizumab in patients with inflammatory bowel disease

R. Fernández ${ }^{1, *}$, G. Sánchez ${ }^{1}$, L. Pedraza', V. Prieto ${ }^{2}$, V. K. Calabuig ${ }^{2}$, E. Fernández ${ }^{1}$, M. J. Otero ${ }^{1}$

${ }^{1}$ Hospital Pharmacy, ${ }^{2}$ digestive Unit, Complejo Asistencial Universitario De Salamanca, Salamanca, Spain

Background and objective: There are few data available on the reallife pharmacokinetic and pharmacodynamic features of vedolizumab, a monoclonal antibody approved for the treatment of moderate to severe inflammatory bowel disease (IBD). There is only limited evidence available about optimum target drug levels to maintain clinical and endoscopic remission.

The aim of this study was to use our experience with therapeutic drug monitoring (TDM) for vedolizumab to determine target trough drug levels for the maintenance phase in patients with IBD.

Setting and method: We conducted a prospective study of IBD patients treated with vedolizumab in maintenance phase and followed by TDM from December 2017 until March 2018.

Clinical information was collected from the patients' medical records. Serum vedolizumab trough levels in steady state (SVTLs) were measured by ELISA.

Main outcome measures: Effectiveness was measured by clinical responses obtained by reviewing the values of inflammatory markers [C-reactive protein (CRP) and fecal calprotectin (FC)] and colonoscopies findings.

After distributing the collected SVTLs data into quartiles (Q), we evaluated the percentage of clinical response for each group.

Results: 24 patients with active IBD were enrolled, 14 patients with ulcerative colitis and 10 patients with Crohn's disease. The average (SD) age was 53.6 (15.7) years. Drug interval in maintenance phase was 8 weeks for 21 patients, 6 weeks for 2 patient, and 4 weeks for 1 patient.

Each SVTLs quartile had 6 patients and frequency ranges were as follows: < 3.5-7.1 mcg/ml (Q1), $8.6-13.8 \mathrm{mcg} / \mathrm{ml}(\mathrm{Q} 2), 14.3-19.5$ $\mathrm{mcg} / \mathrm{ml}(\mathrm{Q} 3)$ and $20.1-52.6 \mathrm{mcg} / \mathrm{ml}(\mathrm{Q} 4)$.

The rates of response per quartile were $50.0 \%$ in Q1 and Q2 and $83.3 \%$ in Q3 and Q4. The median (range) CRP and FC per quartile 
were $0.21(0.11-0.62) \mathrm{mg} / \mathrm{dL}$ and $201(110-1720) \mathrm{mg} / \mathrm{kg}$ in Q1, $0.28(0.05-1.61) \mathrm{mg} / \mathrm{dL}$ and $119(0-260) \mathrm{mg} / \mathrm{kg}$ in $\mathrm{Q} 2,0.05(0-0.54)$ $\mathrm{mg} / \mathrm{dL}$ and $21(0-300) \mathrm{mg} / \mathrm{kg}$ in $\mathrm{Q} 3$ and $0.07(0-0.36) \mathrm{mg} / \mathrm{dL}$ and $50(0-145) \mathrm{mg} / \mathrm{kg}$ in $\mathrm{Q} 4$.

Conclusion: During the maintenance phase with vedolizumab, patients who achieved SVTLs beyond $14 \mathrm{mcg} / \mathrm{mL}$ had improved inflammatory markers and showed better clinical response. TDM can be helpful to avoid underexposure to the drug and increase its efficacy. Larger studies need to be done to determine ideal maintenance phase drug levels.

Disclosure of Interest: None Declared.

TDMP004: Evaluation of the pharmacokinetics of amikacin in neutropenic cancer patients: influence of cancer pathology and chemotherapy

M. Tinoco ${ }^{1}$, M. Rocha $^{2}$, A. Sousa ${ }^{1}$, A. Falcão ${ }^{1,3}$, A. Fortuna ${ }^{1,3, *}$

${ }^{1}$ Faculty of Pharmacy, University of Coimbra, ${ }^{2}$ Pharmaceutical Services, Hospital and University Centre of Coimbra, EPE, ${ }^{3}$ CIBIT Coimbra Institute for Biomedical Imaging and Tanslational Research, University of Coimbra, Coimbra, Portugal

Background and objective: Cancer patients often develop neutropenia, increasing their susceptibility to suffer bacterial infections. Amikacin is prescribed to avoid gram-negative infections. It has concentration-dependent activity and post-bacterial effect with high risk of trough-dependent nephrotoxicity. As neutropenic cancer patient exhibits altered pharmacokinetic (PK) perfiles, concerns exist that current doses may not reliably result in the achievement of target peak plasma concentration $\left(\mathrm{C}_{\max }\right)$ and minimal trough plasma levels $\left(\mathrm{C}_{\text {min }}\right)$.

This study aimed at characterizing amikacin PK in neutropenic cancer patients and investigating if it is different from neutropenic non-oncologic patients. Influence of chemotherapy on amikacin PK was also assessed.

Setting and method: A retrospective study was carried out enrolling neutropenic patients older than 18 years old and admitted in Coimbra Hospital and Universitary Centre in Portugal, undergoing therapy with amikacin and with at least one pair of PK concentrations. To evaluate the influence of tumor diseases on amikacin PK, two groups were created: test (354 cancer patients) and control (275 non-cancer patients). Using Abbottbase PKS software, PK parameters were estimated based on amikacin $\mathrm{C}_{\min }$ and $\mathrm{C}_{\max }$ observed after extended interval administration (EIA). To evaluate the impact of chemotherapy, subpopulations of test group were created considering the interval time between chemotherapy and amikacin (IT $\mathrm{c}_{\mathrm{c}-\mathrm{a}}$ ) administration: simultaneously, $<15,[15-30],[31-90]$ and $>90$ days. Cancer and non-oncologic (sub)populations were compared by ANOVA or t-students tests (SPSS ${ }^{\circledR}$ ).

Main outcome measures: $\mathrm{C}_{\max }$ and $\mathrm{C}_{\min }$, half-life $\left(\mathrm{t}_{1 / 2}\right)$ time and clearance (CL) of amikacin.

Results: Amongst the 562 EIA plasma monitoring measurements within the test group, the prescribed daily dose was 1162.50 (500-2000) $\mathrm{mg}$, resulting in $\mathrm{C}_{\max } /$ Dose and $\mathrm{C}_{\min } /$ Dose ratios of $0.0352 \pm 0.0085 \mathrm{~L}$ and $0.0014 \pm 0.0001 \mathrm{~L}$, respectively. In opposition, the 373 EIA monitorizations from control group demonstrated that patients were administered with lower mean daily doses [868.56 (250-1750) $\mathrm{mg}$ ], revealing statistical differences $(p<0.05)$ when regarding $\mathrm{C}_{\max } /$ Dose ratio $(0.0491 \pm 0.0203 \mathrm{~L})$ and $\mathrm{C}_{\min } /$ Dose ratio $(0.0094 \pm 0.0416 \mathrm{~L})$. Although cancer patients were administered with higher daily doses of amikacin, they presented lower values of $\mathrm{C}_{\min }$ and similar $\mathrm{C}_{\max }$ values of non-oncologic patients after amikacin EIA. More than $60 \%$ of oncologic patients presented $\mathrm{C}_{\max }$ values underdosified. $\mathrm{T}_{1 / 2}$ of amikacin was lower in oncologic patients while $\mathrm{CL}$ was higher $(3.4733 \pm 1.0767$ vs $2.7778 \pm 1.4758 \mathrm{~mL} / \mathrm{min}) . \mathrm{CL}$ decreased as the $\mathrm{IT}_{\mathrm{c}-\mathrm{a}}$ enhanced $(3.9324 \pm 2.2885$ vs $2.6770 \pm 1.0990 \mathrm{~mL} / \mathrm{min}$ ) while $\mathrm{t}_{1 / 2}$ increased from 5.26 to $8.17 \mathrm{~h}$. However, volume of fluid therapy remained unchanged.

Conclusion: Amikacin was eliminated faster from the oncologic patient body, requiring higher doses to be effective. Moreover, attention must be taken if chemotherapy has been administered in the previous 3 months because it influences the PK and pharmacological effect of amikacin.

Disclosure of Interest: None Declared.

TDMP005: Monitoring lacosamide, levetiracetam, zonisamide in plasma of epileptic patients: personalizing the pharmacotherapy of levetiracetam

J. Gonçalves ${ }^{1}$, J. Paulino¹, F. Gouveia ${ }^{1}$, A. Silva ${ }^{2}$, F. Sales ${ }^{2}$, A. Falcão ${ }^{1,3}$, A. Fortuna ${ }^{1,3, *}$

${ }^{1}$ Faculty of Pharmacy of University of Coimbra, ${ }^{2} \mathrm{Hospital}$ and University Centre of Coimbra, EPE, ${ }^{3}$ CIBIT - Coimbra Institute for Biomedical Imaging and Tanslational Research, University of Coimbra, Coimbra, Portugal

Background and objective: Epilepsy affects more than 50 million people worldwide. In spite of the wide variety of new-generation antiepileptic drugs (AEDs) marketed, 30\% of epileptic patients develop refractory epilepsy. Although new AEDs have larger therapeutic ranges, they exhibit high inter- and intra-variability. This reality appeals for a personalized treatment based on the concept of individual therapeutic concentrations.

The present work aimed at evaluating plasma concentrations (PCs) of lacosamide (LAC), levetiracetam (LEV) and zonisamide (ZNS) in epileptic patients; characterizing pharmacokinetics of LEV and evaluate if it is influenced by body mass index (BMI), drug load and concomitant AED administration.

Setting and method: Blood samples were collected at stationary state from 46 patients (27 female and 19 male; mean age of 36 years old, ranging 19-67; mean BMI of $27.26 \mathrm{~kg} / \mathrm{m}^{2}$, ranging 19.10-37.11) admitted between September 2017 and March 2018 in the Epilepsy Integrated Centre of the Hospital and University Centre of Coimbra (Portugal). All of them were under treatment with LAC, LEV and/or ZNS. PCs were determined applying the reproducible innovative HPLC-DAD technique in-house developed and validated. Demographic data, daily dose, co-administered drugs and drug load were also collected for patients treated with LEV. Clearance (CL) was estimated in accordance to population models. Statistical analysis was performed by using SPSS software.

Main outcome measures: According to the AEDs reference ranges, PCs were classified as sub-therapeutic, therapeutic and supra-therapeutic. PCs of LEV were normalized per daily dose and patient weight. The effect of BMI, drug load and co-administration of inducer/inhibitors AEDs on normalized PCs and CL of LEV was assessed.

Results: Among the patients under investigation, 33 were administered with levetiracetam while only 6 and 16 received LAC and/or ZNS, respectively. A total of 79 PCs was determined; no supratherapeutic concentrations were detected. LEV was within the therapeutic range in only $36.4 \%$ of the cases, while $50.0 \%$ and $53.6 \%$ of LAC and ZNS were within, respectively.

In obese patients (BMI $\geq 30 \mathrm{mg} / \mathrm{kg}$ ), normalized PCs of LEV was significantly lower $(0.0326 \pm 0.0192)$ than in those with BMI between $25-30 \mathrm{mg} / \mathrm{kg}(0.0667 \pm 0.0287)$ and $\mathrm{BMI} \leq 25 \mathrm{mg} / \mathrm{kg}$ $(0.0766 \pm 0.0288)$ as well as $\mathrm{CL}(6.30 \pm 0.82,5.05 \pm 1.01$ and $4.97 \pm 0.73 \mathrm{~L} / \mathrm{h}$, respectively). Patients with drug load higher than 3.0 revealed higher normalized PCs $(0.0737 \pm 0.0276$ vs $0.0497 \pm 0.0351)$. Co-administration of typical inducer considerably 
decreased trough normalized PCs $(0.0589 \pm 0.0288)$ while inhibitorS increased $(0.0692 \pm 0.0269)$.

Conclusion: The high frequency of underdosing leading to low PCs of LAC, LEV and ZNS was demonstrated, supporting the importance of monitoring and individualizing the pharmacotherapy of these drugs.

BMI, drug load and inducer/inhibitors co-administration influenced the PCs and clearance of LEV, and must be considered when personalizing the pharmacotherapy of LEV.

Disclosure of Interest: None Declared.

\section{TDMP006: Application of pharmacokinetic monitoring} of amikacin in its pharmacological effects

\section{A. Andrade ${ }^{1}$, M. Rocha ${ }^{2}$, A. Falcão ${ }^{1,3}$, A. Fortuna ${ }^{1,3, *}$ \\ ${ }^{1}$ Faculty of Pharmacy of University of Coimbra, ${ }^{2}$ Pharmaceutical Services, Hospital and University Centre of Coimbra, EPE, ${ }^{3} \mathrm{CIBIT}-$ Coimbra Institute for Biomedical Imaging and Tanslational Research, University of Coimbra, Coimbra, Portugal}

Background and objective: Amikacin has a concentration-dependent activity against Gram positive bacteria and presents post antibiotic effect, making it a first-line pharmacological option against infections of multi-drug resistant bacteria. Although due to its pharmacokinetic characteristics and short therapeutic range, amikacin should be subjected to therapeutic drug monitoring (TDM), it is not always performed as it requires economic efforts and specialized personal skills.

The objective of the present work was to evaluate the influence of the errors detected throughout the TDM protocol with the efficacy and toxicity of amikacin.

Setting and method: The retrospective study herein presented included 576 patients with at least 18 years old, admitted in Coimbra Hospital and Universitary Centre in Portugal between January 2008 and October 2016. All were under treatment with amikacin and with at least one pair of plasma concentrations. Subjects without bacterial infection confirmed by microbiological analysis were excluded. Oncologic patients $(n=354)$ were analysed separately from nononcologic patients $(n=222)$.

Main outcome measures: The errors throughout the TDM process were classified as dosing faults, dosage adjustment failures, failure to take amikacin and administration registration failure.

Clinical outcomes assessed included the creatinemia and creatinine clearance; total dose administered per day; total cumulative dose; number of days to attain leukocyte stabilization; number of days of treatment and hospitalization.

Results: As the number of errors increases, higher was the treatment duration: in no-oncologic population, it enhanced from $9.27 \pm 4.20$ to $20.60 \pm 19.39$ days while, in oncologic patients, the increment was from $10.07 \pm 4.98$ to $14.54 \pm 5.66$ days. Similarly, the length of patient admission also increased (in no-oncologic group, from $34.22 \pm 20.34$ to $64.18 \pm 51.82$ day; in oncologic group, from $49.12 \pm 52.48$ to $66.37 \pm 90.30$ days), and, consequently, the total cumulative administered amikacin dose also enhanced (from $8.84 \pm 4.59$ to $19.11 \pm 17.69 \mathrm{~g}$ in no-oncologic patients and $11.87 \pm 6.99 \mathrm{~g}$ to $17.07 \pm 8.77 \mathrm{~g}$ in oncologic patients). Furthermore as higher is the number of error, longer was the required time to achieve leucocytes stabilization (no-oncologic group: $4.46 \pm 4.83$ increased to $6.08 \pm 8.26$ days; oncologic group: $6.97 \pm 4.00$ increased to $12.20 \pm 9.56$ days) as well as creatinemia. Importantly the number of deaths also enhanced (no-oncologic: 23.53-34.00\%; oncologic: $19.40-29.63 \%$ ).

Conclusion: After analysing both patient groups, it was observed that TDM implementation with fewer errors was associated with faster leucocyte stabilization and hence a better therapeutic response, independently of the infection site. Moreover a well-executed TDM decreases the treatment time and total dose administered, and lower creatinemia. These facts suggest that a TDM implementation with fewer errors prompt a safer therapeutic response, reducing the costs associated with treatment and hospitalization.

Disclosure of Interest: None Declared.

TDMP007: Levetiracetam therapy individualization: predictive performance of three pharmacokinetic models

R. Silva ${ }^{1,2}$, J. Paulino ${ }^{1}$, A. Almeida ${ }^{3}$, J. Gonçalves ${ }^{1}$, A. Silva ${ }^{2}$, F. Sales ${ }^{2}$, A. Falcão ${ }^{1,4}$, A. Fortuna ${ }^{1,4, *}$

${ }^{1}$ Faculty of Pharmacy of Univsersity of Coimbra, ${ }^{2}$ Hospital and University Centre of Coimbra, EPE, ${ }^{3} \mathrm{CIVG}$ - Centro de Investigação Vasco da Gama. Escola Universitária Vasco da Gama, ${ }^{4}$ CIBIT Coimbra Institute for Biomedical Imaging and Tanslational Research, University of Coimbra, Coimbra, Portugal

Background and objective: Levetiracetam (LEV) is a new antiepileptic drug widely used in the treatment of focal and generalized epilepsy, particularly due to its pharmacokinetic and safety profiles. Nevertheless it has significant inter- and intra-individual variability that justifies its therapeutic drug monitoring (TDM) in clinical practice. TDM is expected to be a useful tool to individualize and adjust the dosing regimens of LEV in accordance to the particular conditions of each patient. The availability of validated population pharmacokinetic studies is a requirement to perform adequately TDM.

This study aimed at evaluating the predictive performance of three pharmacokinetic models to estimate plasma concentrations of LEV in adult Portuguese epileptic patients.

Setting and method: A total of 48 LEV plasma concentrations obtained from 38 adult epileptic patients (14 male and 24 female, age: $32.2 \pm 14.3$ years) admitted to the Refractory Epilepsy Reference Centre of the Hospital and University Centre of Coimbra (Portugal) were included. The plasma concentrations of LEV were determined by a validated plasma liquid-liquid extraction followed of high performance liquid chromatography with diode array detection. Demographic, analytical and therapeutic data of each patient were collected.

Volume of distribution and clearance were estimated for each patient applying three pharmacokinetic models (Model A, Model B and Model C). The co-variates included. In Model A: weight and coadministration of antiepileptic drugs that are metabolic inducers; in Model B: weight, daily dose and glomerular filtration rate (GFR); in Model C: weight and GFR. GFR was estimated based on the Modification on Diet in Renal Disease equation. A one-compartment model with first order absorption and elimination were applied to estimate LEV plasma concentrations.

Main outcome measures: Predictive performance of each pharmacokinetic model was given by accuracy and precision that are, respectively, the mean prediction error (ME) and the mean squared prediction error (MSE).

Results: Model A was the one with the lowest absolute value of ME $(0.99 \mathrm{mg} / \mathrm{L})$ and the lowest value of MSE $\left(8.91 \mathrm{mg}^{2} / \mathrm{L}^{2}\right)$, suggesting to be the most accurate and precise model to estimate LEV plasma concentrations. Similarly, Model C exhibited a slight overprediction of LEV plasma concentrations, presenting a low absolute value of ME $(1.14 \mathrm{mg} / \mathrm{L})$ and $\mathrm{MSE}\left(10.73 \mathrm{mg}^{2} / \mathrm{L}^{2}\right)$. In opposition, Model B showed an underprediction of LEV plasma concentrations presenting ME and MSE values of $-2.85 \mathrm{mg} / \mathrm{L}$ and $25.33 \mathrm{mg}^{2} / \mathrm{L}^{2}$, respectively. Conclusion: The results herein presented demonstrated that Model A and Model $\mathrm{C}$ are accurate and precise to estimate LEV plasma 
concentrations in the study population. These models are expected to be successfully employed in TDM of LEV, designing and adjusting LEV dosing regimens, in order to, obtain therapeutic and safe plasma concentrations.

Disclosure of Interest: None Declared.

TDMP008: Genetic characterization of the CYP2C9, CYP2C19, CYPD6 isoenzymes and P-glycoprotein in portuguese patients with depression

\section{P. Magalhães ${ }^{1,2}$, G. Alves ${ }^{3}$, A. Llerena ${ }^{4}$, A. Fortuna ${ }^{1,2, *}$,} A. Falcão ${ }^{1,2}$

${ }^{1}$ Faculty of Pharmacy of University of Coimbra, ${ }^{2}$ CIBIT - Coimbra Institute for Biomedical Imaging and Translational Research, University of Coimbra, ${ }^{3}$ CICS-UBI - Health Sciences Research Centre, University of Beira Interior, Coimbra, Portugal, ${ }^{4} \mathrm{CICAB}$, Clinical Research Centre, Extremadura University Hospital and Medical School, Badajoz, Spain

Background and objective: Cytochrome P450 (CYP) 2C9, CYP2C19, CYP2D6 and P-glycoprotein (P-gp, encoded by $A B C B 1$ gene) are key players of drug metabolism and pharmacokinetics, including antidepressant drugs. However, these proteins are highly polymorphic and numerous genetic variants have been identified as clinically relevant sources of inter-individual variability. The functional activity of such proteins (phenotype) can impact the drug outcomes and the genotype-predicted phenotype ( $\mathrm{gPH})$ is a determinant factor in the definition and prediction of the actual phenotype and phenotype-drug outcomes correlations.

This work aimed to characterize from a genetic perspective a cohort of Portuguese depressive patients at the level of CYP2C9, CYP2C19, CYP2D6 and P-gp.

Setting and method: This work was part of the GnG-PK/PD-AD study, a real-world clinical study focused on the evaluation of the impact of genetic and non-genetic factors on the pharmacokinetics and pharmacodynamics of antidepressant drugs. A total of 182 Portuguese patients were prospectively recruited and genotyped for the CYP2C9, CYP2C19, CYP2D6 and P-gp (ABCB1) genes.

Main outcome measures: Genotype and gPH. CYP genotypes were translated into the Activity Score System, which was then used to determine the individual $\mathrm{gPH}$ : poor metabolizer ( $\mathrm{gPM})$, intermediate metabolizer (gIM), extensive metabolizer (gEM) or ultra-rapid metabolizer (gUM). Haplotype analysis was performed for the $A B C B 11236 C>T, 2677 G>T / A$ and $3435 C>T$ single nucleotide polymorphisms (SNPs).

Results: The frequencies of the SNPs and alleles in study were similar to those described for Caucasians $(p>0.05)$. Amongst the $182,71,90,145$ and $146(39.0 \%, 49.5 \%, 79.7 \%$ and $80.2 \%)$ displayed a non-wild-type genotype for the CYP2C9, CYP2C19, CYP2D6 and P-gp, respectively. We found $111(61.0 \%)$ gEMs, 67 (36.8\%) gIMs and $4(2.2 \%)$ gPMs for CYP2C9; $50(27.5 \%)$ gUMs, $102(56.0 \%)$ gEMs, $26(14.3 \%)$ gIMs and 4 (2.2) gPMs for CYP2C19; and 9 (4.9\%) gUMs, $81(44.5 \%)$ gEMs, $79(43.4 \%)$ gIMs and $13(7.1 \%)$ gPMs for CYP2D6. At the level of $A B C B 1,44$ out of the 182 patients (24.2\%) presented homozygous wild-type haplotypes, 100 out of 182 (54.9\%) heterozygous wild-type haplotypes and 38 out of 182 $(20.9 \%)$ both copies of variant haplotypes.

Conclusion: Genetic variations related to drug metabolism and pharmacokinetics are common in the ordinary clinical setting of treatment of depression, namely in the Portuguese population, and their therapeutic implications must to be studied considering nongenetic factors as well.

Disclosure of Interest: None Declared.
TDMP009: Pharmacokinetic monitoring of high-dose methotrexate in primary central nervous system lymphoma: a descriptive case series study

R. Garcia-Fumero ${ }^{1, *}$, S. Cano-Dominguez ${ }^{1}$, S. Guijarro-Herrera ${ }^{1}$, L. Gutierrez-Zuñiga ${ }^{1}$, C. Alarcon-Payer ${ }^{1}$, A. Jimenez-Morales ${ }^{1}$

${ }^{1}$ Servicio de Farmacia, Hospital Virgen de Las Nieves, Granada, Spain

Background and objective: Primary central nervous system lymphoma (PCNSL) has been always associated with HIV or other immunocompromised patients. In the last decades, however, its incidence has risen among immunocompetent patients reaching a peak in the sixth and seventh decades of life. Although its management remains ill-defined, a multi-agent chemotherapy regimen including high-dose methotrexate (HDMTX) of $3 \mathrm{~g} / \mathrm{m}^{2}$ seems to be effective and well-tolerated when pharmacokinetic criteria are considered. The aim of this study is to describe the influence of methotrexate pharmacokinetics in the management of PCNSL in a tertiary hospital.

Design: A descriptive case series study was conducted including every patient presenting PCNSL and receiving first HDMTX between January 2017 and January 2018. The variables collected from medical records were: age, sex, body surface area (BSA), creatinine clearance prior to therapy, methotrexate dose (standard $=3 \mathrm{~g} / \mathrm{m}^{2}$ ) and number of doses, methotrexate elimination half-life and elimination time. Risk of intoxication was considered, by clinical consensus, when methotrexate elimination half-life, calculated with plasma concentration values of the sixth and twelfth hours from the beginning of infusion, exceeded $3.5 \mathrm{~h}$. Methotrexate elimination was stablished when plasma concentration was $\leq 0.1 \mu \mathrm{mol} / \mathrm{L}$.

Results: Four male patients with a median age of 67 years old and 13 corresponding HDMTX administrations were recruited. None of them presented a diminished creatinine clearance prior to receive HDMTX. However, two patients (50\%) took half of the standard dose as first HDMTX due to patients' frailty and one of them also needed a second dose reduction, receiving $33 \%$ of the standard dose. The median number of doses was 3 and the median elimination half-life was $2.94 \mathrm{~h}$. Three patients $(75 \%)$ showed risk of intoxication and it was always seen in the last HDMTX given, leading to prolonged elimination times (up to $400 \mathrm{~h}$ in the worst-case scenario) and to an early leucovorin administration.

Conclusion: PCNSL is a rare but aggressive disease that involves HDMTX use. Pharmacokinetic monitoring of methotrexate is essential to prevent and confront its toxicity. Nevertheless, results in drug monitoring must be considered with caution because they can be unrepeatable in the same patient under the same conditions, as elimination half-life values have shown in our cohort.

Disclosure of Interest: None Declared.

TDMP010: Personalised treatment of epilepsy: the impact of routine use of therapeutic drug monitoring in patients with juvenile myoclonus epilepsy to control for pharmacokinetic variability, interactions and poor adherence

\section{Flogstad ${ }^{1}$, M. Syvertsen ${ }^{2}$, A. Baftiu ${ }^{3}$, C. Johannessen Landmark ${ }^{4}$,*}

${ }^{1}$ Pharmacy, Oslo Metropolitan University and The Norwegian University of Science and Technology, oslo, ${ }^{2}$ Department of Neurology, Vestre Viken, Drammen Hospital, Drammen, ${ }^{3}$ Pharmacy, Oslo Metropolitan University, ${ }^{4}$ Pharmacy, Oslo Metropolitan University and the National Center for Epilepsy, Oslo University Hospital, Oslo, Norway 
Background and objective: Patients with juvenile myoclonus epilepsy (JME) often have a life-long need for antiepileptic drugs. Many experience uncontrolled seizures and have challenges with adverse effects and variable adherence. Implementation of therapeutic drug monitoring (TDM) contributes to individualisation of their pharmacotherapy. The purpose of this study was to investigate the impact of implementation of TDM in patients with JME to control for pharmacokinetic variability, interactions and poor adherence to antiepileptic drugs for improved personalised treatment.

Setting and method: Retrospective laboratory data based on response from a questionnaire about use of antiepileptic drugs and adherence from patients with JME recruited from Drammen hospital were collected from the TDM-database at the National Center for Epilepsy and Drammen hospital in Norway, 1997-2018. The blood samples were drawn drug-fasting in the morning at assumed steadystate. The study was approved by the Regional Ethics Committee.

Main outcome measures: TDM-data, i.e. antiepileptic drugs in use, doses, measured serum concentrations, time of last dose intake and drug sampling time. Concentration/dose (C/D)-ratios were used to express pharmacokinetic variability.

Results: Data from 80 patients with JME with TDM-data (range 1-61 samples) were included; 49/31 women/men aged 14-39 (mean 25) years, 53(66\%) had myoclonic/generalised seizures within the last year. The most commonly used drugs were valproate $(n=44)$ and lamotrigine $(n=43)$, both involved in numerous interactions, and levetiracetam $(\mathrm{n}=29)$. Inter-individual pharmacokinetic variability in C/D-ratios was extensive, > 10-fold for these drugs. In 12\% (32/ 270) the serum concentrations were below the reference ranges and often variable. Implementation of TDM at least once every year was noted in $50 \%$ of patients and appreciated by $70 \%$. Variable adherence was reported in $29 \%$; $13 \%$ intentionally sometimes/often.

Conclusion: Routine use of TDM of antiepileptic drugs in patients with JME elucidates reasons for sub-optimal treatment in $2 / 3$ of the patients, e.g. low serum concentrations correlating to poor adherence, extensive pharmacokinetic variability and susceptibility to drug interactions. Close monitoring with TDM in patients with JME contributes to an improved personalised approach in the treatment of epilepsy and was highly appreciated by the patients.

Disclosure of Interest: None Declared.

TDMP011: French and German translation of the ABC taxonomy for medication adherence

A. Lehmann ${ }^{1,2, *}$, M. S. Haag ${ }^{3}$, K. E. Hersberger ${ }^{3}$, M. P. Schneider ${ }^{4,5,6}$, A. Gauchet ${ }^{1,7}$, B. Vrijens ${ }^{8}$, I. Arnet $^{3}$, B. Allenet ${ }^{1,2}$

${ }^{1}$ TIMC-IMAG UMR 5525/ThEMAS, University of Grenoble Alps, ${ }^{2}$ Pharmacy Department, Grenoble Teaching Hospital, Grenoble, France, ${ }^{3}$ Pharmaceutical Care Research Group, University of Basel, Basel, ${ }^{4}$ Community Pharmacy, School of Pharmaceutical Sciences, University of Geneva, Geneva, ${ }^{5}$ Community Pharmacy, School of Pharmaceutical Sciences, ${ }^{6}$ Community Pharmacy, Department of Ambulatory Care \& Community Medicine, University of Lausanne, Lausanne, Switzerland, ${ }^{7}$ Interuniversity Laboratory of Psychology (LIP)/PSOCAR Team, University of Grenoble Alps, Grenoble, France, ${ }^{8}$ AARDEX Group, Visé, Belgium

Background and objective: The $\mathrm{ABC}$ taxonomy on medication adherence proposed seven terms (adherence, initiation, implementation, discontinuation, persistence, management of adherence, adherence related sciences) with corresponding definitions (Vrijens et al. 2012). In other languages than English, different terms and definitions of medication adherence are still in use.

We aim to translate the ABC taxonomy in French (F) and German (D) without questioning the original meaning of the definitions. Our objective is to offer a standardized vocabulary for medication adherence to researchers and clinicians.

Design: A literature search in PubMed and a manual search were conducted in F and D to extract existing translations of the seven terms and their definitions. A Delphi method with online survey was used. The expert panel consisted of members of ESPACOMP, the international society dedicated to adherence research since 20 years; professionals recommended by ESPACOMP members, and corresponding authors who had published within the last 5 years articles about medication adherence in French or German. Consecutive rounds were organized until consensus was reached for the proposed translation of terms and definitions. Consensus was defined according to acceptance rate as strong ( $\geq 95 \%$ ), consensus (75-94\%) or moderate $(\geq 50-74 \%)$.

Results: The literature searches were performed on 01.08.2016 (F) and 14.02.2017 (D). Three consecutive rounds sufficed to obtain consensus for all German terms and definitions from 25 experts. First round was initiated on 18.11.2016 (F) and 14.04.2017 (D) with 3'059 ESPACOMP members, and with 17 (F) and 19 (D) corresponding authors; 2nd round on 26.07.2017 with 40 experts (D) and on 31.10.2017 with 62 experts (F); 3rd round on 26.10.2017 with 31 experts (D) and on 17.4.2018 (F) with 26 experts. The French 3rd round is still in process. The preferred F and D terms for "Adherence to medication" are "Adhésion médicamenteuse" (to be confirmed) and "Medikamentenadhärenz". Further results are in process.

Conclusion: With the translations of the ABC taxonomy on adherence in French and German, researchers and healthcare professionals will be able to standardize their vocabulary and harmonize their research projects.

Disclosure of Interest: None Declared.

TDMP012: Plasma drug monitoring implementation of oral targeted therapies in oncology

O. Meynial ${ }^{1, *}$, B. Blanchet ${ }^{2}$, A. Gohari ${ }^{1}$, V. Fallet $^{3}$, A.-M. Ruppert ${ }^{3}$, A. Canellas ${ }^{3}$, A. Lavole ${ }^{3}$, L. Rosencher ${ }^{3}$,

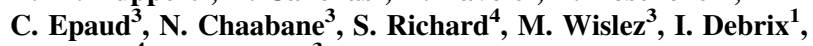
J.-P. Lotz ${ }^{4}$, J. Cadranel ${ }^{3}$

${ }^{1}$ Pharmacy Department, Hôpital Tenon - APHP, ${ }^{2}$ Pharmacology Department, Hôpital Cochin - APHP, ${ }^{3}$ Pneumology Department, ${ }^{4}$ Oncology Department, Hôpital Tenon - APHP, Paris, France

Background and objective: Oral targeted therapies kinase inhibitors (KIs) are being used increasingly in oncology and especially in thoracic oncology. We have set up the progressive use of plasma drug monitoring in routine clinical practice.

The objective of this study is to draw up an inventory of the therapeutic approach based on the plasma trough concentration results and the tolerance of KIs, subjects to many drug-drug interactions (DDI). Design: Between September 1st, 2017 and April 1st, 2018 plasma trough concentrations $\left(\mathrm{C}_{\mathrm{min}}\right)$ of $7 \mathrm{KIs}$, separated into 3 groups according to their pharmacodynamic effects, have been analysed. These therapies are anti-EGFR (afatinib, erlotinib, gefitinb and osimertinib), anti-VEGF (cabozantinib and pazopanib) and anti-ALK (crizotinib) KIs. The 3 evaluated criteria were as follow: patients' plasma exposure, therapeutic approach and tolerance.

Results: 31 patients were included in the study and 39 plasma concentrations have been analysed, within 69\% (27/39) of anti-EGFR KIs. Nearly half $(19 / 39)$ of plasma concentrations were in the recommended range. Out of these 16 patients, 9 didn't have adverses effects. Supratherapeutic concentrations have systematically resulted in dose reduction or a stop of the treatment and all patients have had 1-2 toxicity's grade (4 patients). Conversely, among the infratherapeutic concentrations over $60 \%(9 / 14)$ have resulted in a treatment pursuit without any modification. In this group, oral KIs are well 
tolerated (10/14), only 3 patients have had 1-2 toxicity's grade and 1, 3-4 toxicity's grade. Interpreting DDI was difficult because phytotherapy and aromatherapy used by patients weren't always known. However, 4 DDI have been identified.

Conclusion: This study highlights the benefits of reviewing comedication and measuring plasma drug concentration to individually adjust treatment where necessary.

Clinical pharmacy and drug monitoring could influence teamwork between pharmacists and other healthcare professionals to get patients have better outcomes. Pharmaceutical consultations, already set up in the oncology department, may also find its rightful place in the pneumology department to assist patients with oral anticancer drugs. A prospective study will now be set up to evaluate drug monitoring therapeutic impact associated with overall clinical and pharmaceutical management of the patient.

Disclosure of Interest: None Declared.

TDMP013: Pharmacokinetic behavior and clinical outcomes following a switch from originator to biosimilar CT-P13 infliximab in inflammatory bowel disease: 8 months of real-life experience

J. G. Sanchez-Hernandez ${ }^{1, *}$, N. Martin-Gutierrez ${ }^{1}$, R. FernandezCaballero $^{1}$, N. Rebollo-Diaz ${ }^{1}$, F. Muñoz ${ }^{2}$, A. F. Pordomingo ${ }^{2}$, E. M. Saez-Fernandez ${ }^{1}$, M. J. Otero-Lopez ${ }^{1}$

${ }^{1}$ Pharmacy Service, ${ }^{2}$ Gastroenterology Service, University Hospital of Salamanca, Salamanca, Spain

Background and objective: Biological treatments have improved patient outcomes in inflammatory bowel disease (IBD), but are expensive therapies. Switching patients from originator infliximab to a biosimilar can reduce costs, but real-life data about pharmacokinetics and potential immunogenicity are scarce.

The aim of this study was to prospectively evaluate clinical outcomes (CO) and pharmacokinetic parameters [clearance (CL), and distribution volumes (VD)] immediately after the switch from infliximab originator to biosimilar (CT-P13) and 8 months later in patients with IBD.

Setting and method: From July 2017 until March 2018, we conducted a prospective study of patients with IBD and stable treatment with infliximab, who were switched to infliximab biosimilar treatment (CT-P13). Informed voluntary consent was obtained from all patients. Serum infliximab trough levels (SITLs) in steady state were obtained prior to the switch (T0), immediately after the switch (T1), and 8 months later (T2). SITLs were measured by ELISA (Promonitor ${ }^{\circledR}$ ). Pharmacokinetic analysis was performed using a bayesian approach with a two-compartmental model with first order elimination obtained via a previous analysis of our population (NONMEMTM). CO was obtained by reviewing the evolution of fecal calprotectin (FC) and $\mathrm{C}$-reactive protein (CRP).

The Student's $t$ test was used to statistically analyze the differences between CL and VD, while the non-parametric Wilcoxon test was used for the $\mathrm{CO}$ parameters.

Main outcome measures: Pharmacokinetic parameters: CL, VD.

Clinical outcomes: FC and CRP.

Results: 44 patients ( $54.5 \%$ females) were included with a median (range) age of 42 years (22-68) diagnosed with Crohn's disease $(n=32)$ or ulcerative colitis $(n=12)$. No patients presented anti-drug antibodies. Two patients discontinued therapy for unknown reasons. The average (SD) CL estimated were $0.017(0.004) \mathrm{L} / \mathrm{h} ; 0.016$ $(0.004) \mathrm{L} / \mathrm{h}$, and $0.017(0.003) \mathrm{L} / \mathrm{h}$, at $\mathrm{T} 0, \mathrm{~T} 1$ and $\mathrm{T} 2$, respectively, while the VD estimated were $4.98(0.12) \mathrm{L} ; 5.00(0.13) \mathrm{L}$ and $5.03(0.16) \mathrm{L}$ at the same three times. No differences were found in $\mathrm{CL}$ and VD between $\mathrm{T} 0$ and $\mathrm{T} 1$ and between $\mathrm{T} 0$ and $\mathrm{T} 2$, respectively $(p>0.05)$.
Regarding CO, no differences were found in CRP and FC scores between the three time points $(p>0.05)$.

Conclusion: Our study demonstrates that switching from infliximab originator to biosimilar CT-P13 in a real-life cohort of IBD patients does not lead to changes in pharmacokinetic parameters or mediumterm clinical outcomes. Therefore, we consider the switch in patients with IBD to be safe and effective.

Disclosure of Interest: None Declared.

TDMP014: Development and implementation of TDM service to optimize cyclosporine treatment

\section{Stopinšek ${ }^{1, *}$, P. Drofenik ${ }^{2}$, A. Mrhar ${ }^{3}$}

${ }^{1}$ University Medical Centre Maribor, Pharmacy, Maribor, Slovenia, ${ }^{2}$ Pharmacy, University Medical Centre Maribor, Pharmacy, Maribor, ${ }^{3}$ Faculty of Pharmacy, University of Ljubljana, Ljubljana, Slovenia

Background and objective: To assess the role of the clinical pharmacist by establishing TDM service for cyclosporine and optimizing cyclosporine treatment, in order to determine the applicability of the pharmacokinetic software DoseMe ${ }^{\circledR}$.

Setting and method: 6-month prospective study in a large teaching hospital in Slovenia; all patients with autoimmune diseases on permanent cyclosporine treatment; observation period and intervention period (TDM service); the same parameters were followed during the observation and intervention period; the results were compared at the end of the study.

Main outcome measures: The number of cyclosporine concentration measurements $\left(\mathrm{C}_{0}\right)$, the number of measurements followed by an interpretation, the reaction time needed for a concentration to be interpreted and recorded in the patient's medical documentation, the number of concentrations in therapeutic ranges, the number of days during which patients had therapeutic concentrations of cyclosporine, concomitant use of potentially inappropriate drugs, clinically significant interactions, side effects, mistakes at laboratory controls.

Results: A total of 11 different patients, 2 were discontinued during observation period and 3 new were included during intervention period. Observation period: $24 \mathrm{C}_{0}$ measurements; $19(79 \%)$ were followed by an interpretation, the reaction time was 17.4 days; 9 (38\%) were inside the therapeutic ranges, patients had therapeutic concentrations $37.5 /$ 90 days ( $42 \%$ of time); 6 potentially inappropriate drugs, grapefruit, 1 clinically significant interaction, 3 cases of side effects.

Intervention period: $18 \mathrm{C}_{0}$ measurements; 15 (83\%) were followed by an interpretation, the reaction time was 6.8 days; $12(67 \%)$ were inside the therapeutic ranges, patients had therapeutic concentrations $70 / 90$ days ( $78 \%$ of time); 7 potentially inappropriate drugs, 2 intentionally caused interaction, 2 cases of mistakes at laboratory control. Conclusion: We confirmed the impact of TDM service and the role of clinical pharmacist on the quality of treatment with cyclosporine in patients with autoimmune diseases. During the intervention period, we improved the reaction time and also the number of days, when patients' blood levels of cyclosporine were within the therapeutic range. The pharmacokinetic software DoseMe ${ }^{\circledR}$ was proven as an useful tool when optimizing cyclosporine treatment.

Disclosure of Interest: None Declared.

TDMP015: Tacrolimus intoxication in a kidney transplanted HIV patient: a case report

M. Valle Corpas ${ }^{1}$, P. N. Gómez ${ }^{1}$, I. C. Hidalgo ${ }^{1}$, R. Á. Sánchez ${ }^{1, *}$, P. M. Raya ${ }^{1}$, A. R. Delgado ${ }^{1}$

${ }^{1}$ Servicio de Farmacia Hospitalaria, Hospital San Cecilio, Granada, Spain 
Background and objective: Some of HIV drugs could modify the pharmacokinetics of inmunosupressive drugs. In this case report, we want to warn health professionals about the importance of checking this pharmacokinetic interactions, especially in patients on treatment with potentially toxic drugs.

Design: We have reviewed the clinical reports of this patient and we have searched for analitical data including whole blood drug concentrations and viral loads. For search for information about the pharmacokinetic interactions we've consulted the Interaction Checker of University of Liverpool.

Results: This patient was transplanted on 2013. Since the transplant, he was recibing darunavir/cobicistat $800 \mathrm{mg} / 150 \mathrm{mg}$ daily and dolutegravir $50 \mathrm{mg}$ twice daily as HIV treatment and tacrolimus 7'5 mg a day and micophenolate mofetil $500 \mathrm{mg}$ twice daily. Tacrolimus whole blood concentrations were within therapeutic range (4-11 ng/ml) until September 2016.

In September 2016, the patient went to Emergency Service with hypotension, dizziness, asthenia and hyperglycemia. By that moment, tacrolimus's blood concentration was $123 \mathrm{ng} / \mathrm{ml}$ (a value 10-30 times higher than the therapeutic range in kidney transplanted patients).

In the Pharmakokinetic Area of the Hospital Pharmacy, we checked for drug interactions and we found that darunavir and cobicistat is a CYP3A inhibitor and tacrolimus is a CYP3A substrate so tacrolimus blood concentrations may be increased when taking both drugs.

We proposed to the doctor a tacrolimus dose reduction of $40 \%$ to manage the inteaction. Since this moment, tacrolimus concentrations started to decrease and in 1 month therapeutic levels were acquired, achieving levels maintained within therapeutic range.

Conclusion: A patient interview in the Pharmacy Consult was necessary to detect and prevent this kind of pharmacokinetic interactions with drugs and herbal products. In the same way, a good dose adjustment recommendation of drugs allows us to use them with security even though there were drug-drug interactions.

Disclosure of Interest: None Declared.

DI007: Prescription relevance review in elderly subjects and setting up of tools in order to help doctors, pharmacists and patients

V. Leriverend-Pierre ${ }^{1}$, B. Ledare ${ }^{1}$, B. Marie Dit Dinard ${ }^{1}$, A. Allainmat-Lemercier ${ }^{1, *}$

${ }^{1}$ Pharmacy, Hospital Center, Fougeres, France

Background and objective: In 2017, at the Fougères hospital, a professional practices evaluation have been realized concerning prescription relevance review in 40 patients of geriatric service. This work allowed modifying $90 \%$ (36/40) of the prescriptions. According to these survey results, 58 drugs were stopped after prescription relevance review ( $60 \%$ of modified prescription). Among these stopped drugs, $39.7 \%$ belonged to "nervous system" drugs according to Anatomical Therapeutic Chemical (ATC) classification system. Benzodiazepines and antipsychotics were highlighted as the most involved drugs to modify.

The objective was to develop tools in order to help prescription and prescription analysis regarding psychotropic medications.

Design: All results were exposed to clinicians. The tools' content was decided by common consent. It was constructed from a literature search based on Pubmed, French recommendations (ANSM and HAS), United States (FDA) and Great Britain guidelines (NICE). We also used the American list "Beers criteria", the French list "Laroche" and "STOPP/STARTT" criteria (Screening tool to detect potentially inappropriate prescribing in persons aged 65 or older). The created document was validated by clinicians.
Results: The tool is separated into two parts. The first part is about general medication uses among elderly subjects and describes the appropriate and inappropriate drugs in this population, depending on ATC classification system. The second part is a focus on psychotropic medications, and is classed according to the management of depressive states, anxiety states, and states of agitation split into dementia or confusional origin. For each of these parts, this document detailed the generalities on the drug used, the dosages adjusted on elderly subjects especially in case of renal failure, the side effects, and various remarks including drug interactions, clinical monitoring and possible alternatives. Each drug is mentioned as available or not on the Fougères hospital's drug formulary. After all, the recommendations are exposed according to three levels: "What needs to be ordered: The benefit/risk ratio is favorable", "What should be avoided or proscribed: Iatrogenic risk outweighs benefit because of lack of evidence of effectiveness", "What needs to be discussed on a case-by-case basis: The prescription takes into account the patient's profile (mono or polypathology), his estimated life expectancy and adherence". The document has been distributed to clinicians and pharmacists.

Conclusion: Thanks to this prescription relevance review, we created a tool in order to improve health care quality of the elderly patients. Moreover, this work allowed us to strengthen our links with health professionals like clinicians. Beside these results, this review pointed that a focus on pain management in the elderly and the right use of proton pump inhibitors would be interesting. Thus, we are developing tools destined for clinicians and also patients, in order to inform them on these topics.

Disclosure of Interest: None Declared.

DI008: Safety profile: immunotherapy and Braf/Mek inhibitors in metastasic melanoma

I. Casas Hidalgo ${ }^{1}$, P. Nieto Gomez ${ }^{1}$, A. Rodriguez Delgado ${ }^{1}$, R. Alvarez Sanchez ${ }^{1, *}$, P. Rodriguez Gomez ${ }^{1}$

${ }^{1}$ Farmacia Hospitalaria, Hospital Universitario San Cecilio, Granada, Spain

Background and objective: Background The prognosis of people with metastatic cutaneous melanoma is generally poor. Recently, new classes of drugs (e.g. immune checkpoint inhibitors and smallmolecule targeted drugs) have significantly improved patient prognosis, which has drastically changed the landscape of melanoma therapeutic management. However, this therapy can lead to the appearance of a wide range of adverse effects.

Objective: To evaluate the safety profile of the new classes of drugs used to the treatment of metastatic melanoma: immune checkpoint inhibitors (Nivolumab and Pembrolizumab) as well as small-molecule targeted drugs (BRAF inhibitors: Vemurafenib and Dabrafenib; and MEK inhibitors: Trametinib and Cobimetinib) in a secondary hospital.

Design: Retrospective observational study from January 2016 to March 2018 of all patients treated with immunotherapy, BRAF inhibitors and/or MEK inhibitors. The variables analyzed were: adverse effects and dose reduction/withdrawal associated with the new drugs used for the treatment of metastatic melanoma.

Results: Number of patients: Nivolumab (NIVO) 5 patients; Pembrolizumab (PEMBRO) 3 patients; Vemurafenib (VEMURA) 8 patients; Dabrafenib (DABRA) 14 patients; Trametinib (TRAME) 16 patients and Cobimetinib (COBI) 4 patients.

* adverse effects (\%patients): NIVO $\rightarrow$ cutaneous (20\%), asthenia $(20 \%)$, ankle arthritis (20\%); PEMBRO $\rightarrow$ haematological toxicity $(33 \%)$; VEMURA $\rightarrow$ digestive and hepatic (16\%), facial edema $(12 \%)$, phototoxicity (12\%); DABRA $\rightarrow$ fever (14\%), GI (nausea, vomiting) (14\%) and hepatic (14\%), cutaneous (7\%); 
TRAME $\rightarrow$ cutaneous $(12 \%)$, ocular $(6 \%)$, GI $(6 \%)$, fever $(6 \%)$, hepatic $(6 \%)$, hematologic $(6 \%)$; COBI $\rightarrow$ hepatic $(75 \%)$, GI (diarrhea, nausea) $(50 \%)$, cutaneous $(50 \%)$, facial edema (25\%).

$*$ dose reduction and/or withdrawal (\% patients): NIVO none, PEMBRO none, VEMURA 25\% reduction and 32\% withdrawal, DABRA $36 \%$ reduction and $14 \%$ withdrawal, TRAME $29 \%$ reduction and $21 \%$ withdrawal, COBI $50 \%$ reduction and $25 \%$ withdrawal.

Conclusion: BRAF and MEK inhibitors develop considerable toxicity, which on several occasions led to the reduction of dose and/or withdrawal of the drug. In contrast, although immunotherapy can provoke the development of some side effects it did not lead to any dose reduction and/or withdrawal. However, more robust studies are necessary to confirm these results.

Disclosure of Interest: None Declared.

DI009: Determination of the knowledge of the participants regarding probiotics purchased from community pharmacy

D. Ylddz ${ }^{1}$, K. N. Cengiz ${ }^{1, *}$, B. Okuyan ${ }^{1}$, F. V. İzzettin ${ }^{1}$, M. Sancar ${ }^{1}$

${ }^{1}$ Department of Clinical Pharmacy, Marmara University, Istanbul, Turkey

Background and objective: Currently, the awareness and use of probiotics among patients is increasing. This study was aimed to evaluate participant's level of knowledge regarding probiotics purchased from community pharmacy.

Setting and method: The research was held between November 2017 and April.2018 in a community pharmacy at Istanbul. The study was performed by face to face interview. This study was conducted in clients who came to pharmacy with any reason and willing to participate in this study after informed.

Main outcome measures: A self-structured questionnaire with 28-items was used for data collection.

Results: In this study, the questionnaire was applied to 104 participants. The average age of participants is $34.71 \pm 11.9$. Of them, $56.7 \%$ reported that they used probiotics previously. However, $43.3 \%$ of them declared that they had never used probiotics. When questioning the main benefit of probiotics, $76 \%$ of participants supposed that probiotics could strengthened the immune system and only $54.8 \%$ of them considered benefit of probiotics in treatment of diarrhea. Probiotics users had statistically more belief on effect of probiotics on immune system when compared with probiotics non-user $(p<0.05)$. Female participants had more belief on safe usage of probiotics in children when compared with male participants $(p<0.05)$. Although, $25.0 \%$ of participants reported that they would consult pharmacists for more information regarding probiotics, $41.3 \%$ of them reported that they would consult with their physicians.

Conclusion: According to results of this study, the majority of participants agree that probiotics are used to strengthen the immune system and the participants who use probiotics and female participants in particular believe that probiotics are more likely to have a beneficial effect. The low rate of pharmacy consultation on probiotics could be addressed by further education studies regarding probiotics conducted by pharmacists.

Disclosure of Interest: None Declared.

DI010: Rebound effect occurrence after interruption of a long term proton pump inhibitor (PPI) treatment: a real obstacle to de prescription

C. Debanne $^{1, *}$, I. Dagrenat ${ }^{1}$, A.-C. Desbuquois ${ }^{1}$, M. Boisgontier ${ }^{1}$

${ }^{1}$ Oise, Centre Hospitalier Compiegne-Noyon, Compiegne, France
Background and objective: The PPI class is one of the most prescribed in France, but many prescriptions are improper. Side effects (SE) of PPI highlight the importance of their good use. These misuses have been identified through a study in our hospital, revealing real difficulties to reevaluate long term PPI treatments. One of the limits would be the fear of rebound effects (RE) causing SE.

This study aims to assess the occurrence of RE leading to a resumption of the PPI after the modification of treatment.

Design: Realization of a prospective study with 1-6 months medical monitoring, including institutionalized patients with prescription modification after reevaluation during the previous survey. Many criteria were taken into account: prescription modification in conformity with the pharmaceutical intervention established with a flowchart, maintenance duration of the modification, resumption of a PPI and occurrence of SE.

Results: The previous survey showed 34 reevaluations accepted by the prescriber.

Of the 23 patients with an interruption of treatment, only 1 received a new PPI prescription after the occurrence of RE. All the prescriptions were interrupted more for than 1 month: 14 for $1-3$ months, 4 for 3-6 months and 4 for 6 months or more.

None of the 5 patients with a reduced posology suffered from RE. The actual duration of the treatment interruptions were: 2 months for 3 patients, 3 months for 1 and 5 months for 1patient.

Of the 16 prescriptions changed for If Needed (IN), only 1 has been accepted and maintained for more than 6 months. For 5 patients, the medical decision was an abrupt stop despite the proposition to change for IN by the pharmacist: 2 patients suffered of RE leading to the resumption of the PPI after less than 1 month.

Conclusion: This study shows that PPI treatment interruption is possible: only $8.82 \%$ of RE occurrence were observed. It is necessary to follow certain steps to avoid the occurrence of RE. Two of the resumptions can be imputed to a wrong treatment interruption: a stop instead of a change for IN.

Those results will be presented to the prescribers to improve the PPI prescription reevaluations.

Disclosure of Interest: None Declared.

DI011: Appropriate health information on drug use in renalor liver impairment for physicians, pharmacists and patients

\section{A. Hielema ${ }^{1, *}$, B. Wensveen ${ }^{1}$, N. Everaarts-de Gruyter ${ }^{1}$}

${ }^{1}$ Centre for Information on Medicines, KNMP Royal Dutch Pharmacists Association, The Hague, Netherlands

Background and objective: Many drugs are eliminated by the kidney and/or the liver. In case of dysfunction of these organs, elimination is disturbed and drug toxicity might occur. Patients with renal impairment, dialysis or liver cirrhosis are at risk for adverse events if physicians and pharmacists haven't taken this into account in the therapy. Physicians and pharmacists can prevent this by choosing an alternative drug, or adjusting the dose, or providing additional monitoring. Patients have to be informed when dose adjustment or monitoring during drug use is necessary.

The first objective of this project is to standardize the information on drug use in renal- or liver impairment for physicians, pharmacists, and patients. The second objective is to provide this standardized drug information to physicians, pharmacists, and patients.

Design: Different guidelines on drug use in renal impairment, hemodialysis (intermittent hemodialysis and continuous hemodialysis) and peritoneal dialysis and guidelines on drug use in liver cirrhosis have been collected. Relevant drug information texts have been rewritten and edited for three implementation tools to disseminate the drug information. The first tool is the Informatorium Medicamentorum, the leading drug information database for 
pharmacists in the Netherlands. The second tool is the G-Standaard, the Dutch national drug database for software. This database contains decision support information for medication surveillance by physicians and pharmacists. The database is incorporated in computerized physician order entry (CPOE) and pharmacy information systems in the Netherlands. The last dissemination tool is a drug information website for patients www.apotheek.nl. This website is the most consulted drug information site for the general public in the Netherlands. Information on this website is adapted to the reading level of patients.

Results: Drug information on the use of 650 drugs in renal impairment (including dialysis) is collected and incorporated in the three dissemination tools: Informatorium Medicamentorum, G-Standaard and www.apotheek.nl. The drug information is graded into two groups: 'intervention required' and 'intervention not required'.

Information on the use of 200 drugs in liver cirrhosis is also collected and incorporated in all three dissemination tools. For some drugs, the advice is depending on the severity of liver cirrhosis expressed in Child-Pugh class.

The drug information consists of advice for dose adjustment, monitoring the patient, or choosing an alternative drug.

Conclusion: We provide evidence based drug information for physicians and pharmacists for dose adjustment and other measures in case of renal or liver impairment. At the same time the patient is informed and alerted, if needed, by consulting drug information at www.apotheek.nl. In this way pharmacists, physicians and patients are able to use complementary drug information from the same source.

\section{Disclosure of Interest: None Declared.}

DI012: Evaluation of segurity of Palbociclib in the treatment of metastatic breast cancer

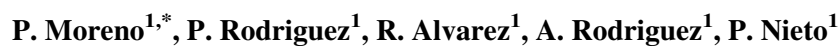

${ }^{1}$ Hospital Universitario San Cecilio, Granada, Spain

Background and objective: The objective is to evaluate and describe the adverse effects produced by palbociclib in patients with metastatic breast cancer in daily clinical practice.

Design: Retrospective observational study. We proceed to review the clinical history of the eight patients treated with palbociclib in our hospital, through the Diraya and Prisma Athos computer programs.

Results: We describe 8 cases with a median age of 44 years, of which 1 is a male $(12.5 \%)$ and 7 are female $(87.5 \%)$. Patients have received treatment for at least 1 month ( 1 complete cycle), with an initial dose of $125 \mathrm{mg}$ daily (for 21 days). Seven cases of neutropenia are detected: 2 of grade I (25\%), 2 of degree II $(25 \%), 2$ of degree III $(25 \%)$ and 1 case of neutropenia grade IV (12.5\%). There are two cases of constipation (25\%). A case with hand-foot syndrome grade II is detected that requires a dose reduction of $125-100 \mathrm{mg}$. In addition, the patient suffers episodes of headaches associated with treatment with palbociclib. 2 patients require temporary suspension of treatment for 1 week $(25 \%)$.

Conclusion: Neutropenia is the most frequent adverse effect, being detected in $87.5 \%$ of patients treated with palbociclib. Treatment with this drug requires close control and constant monitoring to be able to act effectively when adverse effects occur.

Disclosure of Interest: None Declared.

DI013: Interactions between oral anticancer drugs and food, herbal medicine, food supplements...: what to tell to our patients

V. Pelagatti ${ }^{1, *}$, C. Bourrel ${ }^{1}$, N. Fabre ${ }^{2}$, A. C. Le Lamer ${ }^{2}$, F. Puisset ${ }^{1}$, M. Richl $^{3}$, M. Montastruc ${ }^{4}$, A. Eche Gasse ${ }^{3}$, N. Caunes Hilary ${ }^{3}$, V. Flanzy ${ }^{1}$, J.-M. Canonge ${ }^{1}$
${ }^{1}$ Pharmacie, IUCT ONCOPOLE, ${ }^{2}$ UMR 152 Pharma Dev, Université de Toulouse, IRD, UPS, ${ }^{3}$ Soins de Support, ${ }^{4}$ Oncologie médicale, IUCT ONCOPOLE, Toulouse, France

Background and objective: A large number of patients treated with oral anticancer drugs use complementary therapies or specific diets to fight against cancer or to prevent side effects of treatment. Interactions could occur between the anticancer treatment and these therapies or specific diets. Patients inform themselves and obtain treatment by using internet. They don't always talk about it to their doctor or their pharmacist. Moreover, health professionals do not always have the same discourse in dealing with these practices and are often unable to advise patients. Our purpose is to develop a clear information pamphlet for patients that will allow for a correlated communication between health professionals. This document should also encourage patients to talk more with professionals about their specific therapies or specific diets.

Design: From September 2017 to March 2018, a multidisciplinary team consisting of doctors, nurses, dieticians and pharmacists in association with pharmacologists and herbal medicine teachers, have developed a patient information document about the risks of interactions between oral anticancer drugs, diet and complementary therapies. This document has been validated by the quality control unit of the etablishment. The multidisciplinary team worked on interactions of anticancer oral drugs with food, plants, complementary diets and also with gemmotherapy, aromatherapy, apitherapy and homeopathy. Three categories were defined with different color codes: associations to avoid (color red), associations to use with caution (color orange) and associations without risk (color green).

Results: Some foods are to be consumed in moderation as part of a balanced diet. Quantities corresponding to a balanced diet are given to help patients. Herbal medicine is to avoid as many plants have an effect on hepatic metabolism and could theoretically interact with many oral anticancer drugs. Complementary diets are to be used with caution because they may contain plants extracts and also due to the lack of quality control and possible variations in their composition. For lack of data, gemmotherapy, apitherapie and aromatherapy should be used with caution and are not recommended. Finally, homeopathy could be used without risk in $\mathrm{CH}$ or more than $6 \mathrm{DH}$ dilutions.

Conclusion: This document has been recently given by doctors, nurses and pharmacists to patients in our institution during consultation or treatment deliveries. It's also transmitted as part of the community-hospital link to community health professionals. The purpose of this document is to evolve according to the state of knowledge on the risks of interactions.

Disclosure of Interest: None Declared.

\section{DI014: Drug shortages—management in a Medicines Information} Center

\section{S. Ulsø ${ }^{1, *}$, R. S. Nielsen ${ }^{1}$, M. M. Nielsen ${ }^{1}$, M. H. Clemmensen ${ }^{1}$ \\ ${ }^{1}$ Medicines Information Center, The Capital Region of Denmark Hospital Pharmacy, Copenhagen NV, Denmark}

Background and objective: Drug shortages (DS) are a challenge for the Hospital Pharmacy (HP) and may be associated with patient safety risks. The Medicines Information Center (MIC) in the Capital Region of Denmark is often involved in DS in terms of finding alternatives but also in handling issues concerning different storage conditions, stability, administration and compatibility.

The aim of the study was to investigate how often the MIC is involved in the management of DS; what type of DS that requires MIC involvement and what challenges that are solved in this context. We elucidated whether the queries originated from the HP's Procurement Department, the hospitals or other HP staff. 
Setting and method: The MIC answers questions from all healthcare professionals in the Capital Region of Denmark and all queries are registered in a database. Queries regarding DS during 2016/2017 was drawn from the database and manually analyzed with regards to: type of challenge, therapeutic area, dosage form and alternative drug. Further data analysis was performed in Excel.

Main outcome measures: The Number of DS queries received in the MIC, the type of challenges, the therapeutic areas and route of administration involved and alternative drugs found.

Results: A total of 1059 DS of medicines in tender was registered in a national database during 2016/2017. The MIC received 229 queries regarding DS during 2016/2017. The therapeutic areas most often involved were within ATC-group B (18\%), J (15\%) and N (12\%). Drugs for IV administration accounted for $52 \%$ and oral for $19 \%$ respectively. Identification of alternatives accounted for $59 \%$ of the queries, information on delivery for $12 \%$ and stability/storage for $8 \%$. Alternatives were found in $82 \%$ of the cases and authorized, unlicensed and extemporaneous drugs accounted for 53\%, 16\% and $10 \%$ respectively. About $28 \%$ of the queries came from the Procurement Department, 34\% from hospital wards and $8 \%$ from HP staff.

Conclusion: The MIC received 229 queries regarding DS. More than half of the cases involved drugs for IV administration and the most frequent challenge was identification of an alternative drug. The present results emphasize that MIC plays an important role in managing DS of critical pharmaceuticals.

Disclosure of Interest: None Declared.

PE004: Longitudinal evaluation of laxative use among people with intellectual disabilities: data from three waves of a longitudinal study

H. K. Al Mutairi' ${ }^{1, *}$, M. O'Dwyer', M. McCarron ${ }^{2}$, P. McCallion ${ }^{3}$, M. C. Henman ${ }^{1}$

${ }^{1}$ School of pharmacy, ${ }^{2}$ School of Nursing \& Midwifery, Trinity College Dublin, Dublin, ${ }^{2}$ Ireland, ${ }^{3}$ School of Social Work, Temple University, Philadelphia, United States

Background and objective: Constipation is a frequent and concerning condition among people with intellectual disability (ID) and laxatives are the most prevalent treatment. The objective of this study was to evaluate the change in prevalence and patterns of both constipation and laxative use between the waves of a longitudinal study. Setting and method: Data describing participant demographics, selfreported diagnosis of constipation and laxative use were extracted from the Intellectual Disability Supplement to the Irish Longitudinal Study on Ageing (IDS-TILDA), wave 1 (2010-2011), wave 2 (2012-2013) and wave 3 (2016-2017); participants were over 40 years and living in Ireland. Descriptive statistics, bivariate analyses were carried out (1).

Main outcome measures: Prevalence of laxative use (single and multiple) according to demographics, reported constipation and Rome III criteria (2).

Results: From the first wave until wave 3, there was an increase in self-reported constipation of $27 \%$, up to $44.5 \%(n=244)$. The use of laxatives increased by $6.6 \%$ from wave 1 from 37.8 to $44.4 \%$ at wave 3 . The use of $2+$ laxatives has decreased by $11 \%$ since wave 1 . Detailed information about laxative use and Rome III criteria for constipation was available for waves 2 and 3. Intraclass laxative combinations were prevalent in wave 2 and 3 and accounted for $24 \%$ and $20 \%$ of the laxative users, respectively. The use of laxatives alone, without a report of any of the two chronic constipation indicators (self-reported and Rome III criteria), occurred at a similar percentage in the two waves; wave $2 ; 14.2 \%$ and wave $3 ; 13.5 \%$.

Conclusion: Over time the use of laxatives and the reporting of constipation have both increased in parallel among people with ID. A thorough assessment and specialized guidelines for constipation is needed for people with ID because the usage is complicated and the factors that predispose participants to constipation are prevalent. Disclosure of Interest: None Declared.

PE005: Influence of primary care antibiotic prescribing on incidence rates of extended-spectrum $\beta$-lactamase-producing bacteria (ESBLs) in hospitalised patients

M. S. Alnajjar 1,2,*, M. A. Aldeyab ${ }^{3}$, M. G. Scott ${ }^{3}$, M. P. Kearney ${ }^{4}$, G. Fleming ${ }^{3}$, F. Glimore ${ }^{3}$, J. C. McElnay ${ }^{1}$

${ }^{1}$ School of Pharmacy, Queen's University Belfast, Belfast, United Kingdom, ${ }^{2}$ College of Pharmacy, Al Ain University of Science \& Technology, Al Ain, United Arab Emirates, ${ }^{3}$ Medicines Optimisation Innovation Centre, ${ }^{4}$ Area Microbiology Laboratory, Antrim Area Hospital, Antrim, United Kingdom

Background and objective: Use of antibiotics can give rise to the selection of resistant bacteria. The goal of this study was to explore the impact of prior community antibiotic usage on hospital detected ESBL incidence rate, focusing at an individual patient level.

Setting and method: This pharmacoepidemiological study was casecontrol in design, and was carried out within the Antrim Area Hospital in two phases. During the first phase, the controls were matched according to: age (two categories: $16-65$ years and $>65$ years), gender, admission ward, date of admission ( \pm 30 days) and age-adjusted Charlson co-morbidity index score (two categories: 0-2 and $>2$ ). During the second phase, controls were selected randomly from the total population of admissions to the hospital over a 2 year period. Following Univariate analysis, multivariate logistic regression was employed to identify particular antibiotics (and patient-related risk factors) that were independently associated with the hospital detected ESBLs.

Main outcome measures: The primary outcome measure was the impact of antibiotic prescribing (penicillin combinations, fluoroquinolones, second and third generation cephalosporins and macrolides) in primary care on hospital detected ESBLs. The primary outcome measure of phase 2 was the association between matching variables for Phase 1 and other risk factors.

Results: In total, 183 ESBL isolates were recovered from the hospitalised patients within the study site hospital over the 2 year study period. After applying the study inclusion criteria, 98 patients harbouring ESBLs were eligible, and comprised the cases group. E. coli were found to be the most frequently $(75.3 \%)$ detected ESBLs. The majority $(66.3 \%)$ of the ESBL strains were cultured from urine samples. The overall prevalence rate of ESBLs was 0.088 cases/100 bed-days. In phase 1, multivariate analysis revealed that prior exposure to second and third generation cephalosporins $(p=0.004)$ and fluoroquinolones $(p=0.023)$ in primary care was associated with an increased likelihood of ESBLs in inpatients. In phase 2, an independent relationship between an increased risk of infection with ESBLs was associated with: prolonged hospitalisation $(p<0.001)$, being elderly $(p<0.001)$, being female $(p=0.007)$ and having genitourinary disease $(p<0.001)$.

Conclusion: The study identified the independent association between previous exposure (in primary care) to certain antibiotic groups (second and third generation cephalosporins and fluoroquinolones) and the prevalence rate of ESBL-producing bacteria detected in secondary care. 'Non-antibiotic' risk factors, independently associated with the detection of ESBL-producing organisms in hospital were; prolonged hospitalisation, being female, presence of genitourinary disease (as a primary diagnosis) and older age.

Disclosure of Interest: None Declared. 
PE008: Effectiveness of a structured intervention to optimize the use of mirabegron, a $\beta 3$ receptor agonist: a quasiexperimental study

\section{E. Fernández-Liz ${ }^{1, *}$, A. Aranzana ${ }^{1}$, M. E. Barceló ${ }^{1}$, M. J. López-Dolcet ${ }^{1}$, J. Ossó ${ }^{1}$, P. Vivó ${ }^{1}$ \\ ${ }^{1}$ Catalan Institute of Health, Barcelona, Spain}

Background and objective: Overactive bladder is a composite of lower urinary tract storage symptoms. Pharmacological treatment is widely employed despite markedly modest efficacy data, adverse effects, and costs for the health system. Our work aimed to determine the effectiveness of a training activity followed by a reassessment of the prescription of mirabegron to achieve its short and long term deprescribing. Likewise, it was proposed to establish the duration of medication in real clinical practice.

Setting and method: A longitudinal, quasi-experimental study was designed comparing 17 intervention practices with 34 control practices located in Barcelona. Inclusion criteria were patients with mirabegron prescription. The follow-up period was 12 months, from January 1st to December 31st, 2017. A structured intervention was designed consisting of initiatives with general practitioners, urology/ gynaecology specialists, support from health care authorities, and monthly feed-back monitoring to general practitioners.

Main outcome measures: The percentage of change of the number of patients treated and new patients with treatment respect to basal values at T1 (3 months after inclusion), T2 (6 months after inclusion), T3 (9 months after inclusion), and T4 (12 months after inclusion).

Results: We identified 1932 patients using mirabegron during January 2017 . Of the total, $53.8 \%$ were female, and $39.4 \%$ belonged to the intervention group. Overall, the patients' mean age was 72 years. Regarding the initial cohort, a significant reduction in treatment of $56.8 \%$ in the intervention group was observed 12 months after intervention, while in the control group it was $41.4 \%(p<0.001)$. There was also a lower incorporation of new treatments in the intervention group compared to the control (28.1\% vs $50.9 \%)$ $(p<0.001)$. In relation to persistence, the differences between the intervention and control groups were statistically significant by approximately $9,13,15$, and 15 percentage points $(p<0.001)$ at 3,6 , 9 , and 12 months, respectively. In relation to patients with treatment at the beginning and end of the period, there was an increase of $9.2 \%$ in the control group and a decrease of $28.3 \%$ in the intervention group $(p<0.001)$

Conclusion: The structured intervention showed both optimization in the use of mirabegron and facilitation in its deprescribing. When the latter is considered it is necessary to provide clear data on the benefits/ risks of treatment options for patients and their caregivers as such information is a precondition for shared decision-making.

Disclosure of Interest: None Declared.

PE009: Direct oral anticoagulants and their uses in primary health care

\section{S. Herraez-Nieto ${ }^{1, *}$, E. Fernández-Liz ${ }^{2}$, P. Modamion', M. E. Barceló Colomer ${ }^{2}$, E. L. Mariño ${ }^{1}$}

${ }^{1}$ Clinical Pharmacy and Pharmacotherapy Unit, Department of Pharmacy and Pharmaceutical Technology, and Physical Chemistry, Faculty of Pharmacy and Food Sciences, University of Barcelona, ${ }^{2}$ Barcelona Primary Health Care, Catalan Health Institute, Barcelona, Spain

Background and objective: The use of direct oral anticoagulants (DOAC) involves knowledge of clinical situations in which these medicines should be used with caution or are contraindicated. The aim of this study was to determine the degree of implantation of
DOAC in primary health care practice, to describe the pattern of use and to detect areas to improve the prescription in patients with atrial fibrillation.

Setting and method: A cross-sectional study was performed through the records of databases containing relevant clinical information (diagnoses, treatments, explorations and other variables) on the use of DOAC.

Main outcome measures: Appropriateness was evaluated according to the indication, the thromboembolic and hemorrhagic risk, renal function, and the risk of drug interactions.

Results: On a population of 351,732 inhabitants, 9194 (2.6\%) were treated with an oral anticoagulant. $63.8 \%$ with acenocumarol, $36.2 \%$ with DOAC: dabigatran $5.8 \%$, rivaloxaban $16.1 \%$, apixaban $10.9 \%$ and edoxaban $3.4 \%$. The group of age between 70 and 89 years old was identified as the group with the highest consumption of all (72.6\%). $85.5 \%$ of patients had a high risk of suffering a thromboembolic episode (CHAD2DS-VASc $\geq 2$ ), although the risk was around zero in $4.4 \%$ of the patients. $14.7 \%$ had a risk of haemorrhagic episode (HAS-BLED $\geq 3$ ). In patients treated with DOAC, rivaroxaban was the most prescribed drug except for patients older than 90 years old which was apixaban. $518(15.6 \%)$ patients did not have the indication authorized (valvulopathy). Situations that lead to increase the risk of bleeding or atherothrombotic events were found; these events were the following: inadequate medication dose in 1 out of 5 patients, without glomerular filtration rate $(90,2.7 \%)$; without glomerular filtration rate nor plasma creatinine $(89,2.7 \%)$, with neoplasia $(806,24.2 \%)$, with peptic ulcus $(761,22.9 \%)$, with a history of ischemic heart disease $(700,21.0 \%)$, with hepatopathy $(252,7.6 \%)$, with a poor control for patients suffering from severe arterial hypertension $(185,5.6 \%)$, and the concomitant use of drugs which increase the risk of bleeding: ISRS or ISRN $(605,18.2 \%)$, antiepileptics $(402,12.1 \%)$, antiagregants $(306,9.2 \%)$, dronedarone $(36,1.1 \%)$ and NSAID $(32,1.0 \%)$.

Conclusion: In this study DOAC consumption represented $36.2 \%$ of all drug consumption. Clinical situations in which the prescription was susceptible to improvement were identified (patients with valvulopathy, inadequate dosage, without glomerular filtration rate, with poorly controlled hypertension, hepatopathy, neoplasm, ischemic heart disease, peptic ulcer and the risk of pharmacological interactions with antiagregants, SSRI or ISRN).

Disclosure of Interest: None Declared.

PE010: Identifying primary healthcare issues amongst the homeless for preventative and personalised care: a feasibility study using routinely collected datasets

M. Bowen ${ }^{1}$, K. Saunders ${ }^{2}$, A. Yahyouche ${ }^{1}$, T. Marshall ${ }^{1}$, S. Marwick ${ }^{1}$, D. Stewart ${ }^{3}$, V. Paudyal ${ }^{1, *}$

${ }^{1}$ University of Birmingham, ${ }^{2}$ Public Health England, Birmingham,

${ }^{3}$ Robert Gordon University, Aberdeen, United Kingdom

Background and objective: Estimating the prevalence of health conditions amongst the homeless has been historically difficult due to challenges associated with identifying homeless population in routinely collected datasets and issues around reliability with survey methodology. Therefore accurate estimates of prevalence of health conditions in this population are not available. Such data are imperative for service development including preventative services and provision of personalised care. This study explores the feasibility of identifying health problems amongst the homeless using routinely collected datasets from a specialist general practice for homeless population.

Setting and method: Data were extracted from the EMIS patient records database of a specialist homeless general practice in West Midlands of England. Frequency of specific health conditions was 
determined from appropriately coded records of primary care consultations. In addition, Quality and Outcomes Framework Datasets were used.

Main outcome measures: Prevalence of long term health conditions in the homeless in 21 disease areas, practical considerations in estimating prevalence.

Results: Datasets of a total of 928 homeless patients were reviewed. A high majority $(89.5 \%)$ were male with a mean (SD) age of 38.3 (11.5). A total of $452(48.7 \%)$ of patients had at least one chronic conditions with a mean (SD) of 1.7(0.99) chronic conditions. Highest prevalence were observed with alcohol dependence $21.3 \%$, drug dependence $13.5 \%$, depression $11.6 \%$, sexually transmitted infections $9.4 \%$, leg ulcer $6.5 \%$, hepatitis C $6.3 \%$, asthma $4.2 \%$. Nearly one in three had attended hospital Accident and Emergency Departments in the last 12 months $\mathrm{n}=302(32.5 \%)$. Alcohol, drug dependence and $\mathrm{A} \& \mathrm{E}$ attendance were closely interlinked. Limitations to the use of the databases include incomplete recording and limited information around individual patient demography.

Conclusion: A high prevalence of key long term illnesses were observed amongst the users of the specialist homeless healthcare centre. The use of the databases may provide an under-estimation of the prevalence rate. Future studies should consider extracting datasets from individual patient notes from the specialist general practice from homeless population alongside the databases and survey methodology to estimate and triangulate the true prevalence of the health conditions. Given the high prevalence of chronic diseases, tailored preventative services including clinical pharmacy are imperative. Disclosure of Interest: None Declared.

PEC003: An economic study of a potential therapeutic interchange regarding biosimilar products

\section{S. Apikoglu-Rabus ${ }^{1,2}$, B. Arslan ${ }^{3}$, K. N. Cengiz ${ }^{2, *}$ \\ ${ }^{1}$ Turkish Pharmacists' Association, Ankara, ${ }^{2}$ Clinical Pharmacy Department, ${ }^{3}$ year-5 student, Marmara University Faculty of Pharmacy, Istanbul, Turkey}

Background and objective: There has been an exponential growth in development of biologic agents. This tremendous growth is life saving on one hand, causing a serious financial threat to the health care system on the other. Manufacturing of biosimilar products help overcome this financial concern. This study aimed to make an economic analysis of a potential therapeutic interchange scenario regarding biosimilar products and their reference products marketed in Turkey.

Setting and method: The study was conducted using the data regarding the biosimilar products (i.e. insulin glargine, enoxaparin, somatropin, infliximab, filgrastim, epoetin alfa) available in Turkey in 2017. IMS sales data was obtained from the Turkish Pharmacists' Association. Only biosimilar products with the potencies comparable to their reference biologics were taken into analysis. The amount of money that would have been saved (and the number of extra doses that could have been bought with this saved amount) was calculated for a scenario of therapeutic interchange regarding biosimilar products and their reference biologics.

Main outcome measures: The amount of money saved if the scenario "of substitution of any reference biologic with its biosimilar equivalent" were realized.

Results: The results of the calculations yielded savings of $1,424,078,125$ units of insulin glargine; 2,292,512 doses of enoxaparin, 11,252 doses of somatropin, 4239 doses of infliximab and 285,938 doses of filgrastim.

Conclusion: Our results showed that starting the treatment with a biosimilar product in treatment-naïve patients or a therapeutic interchange with biosimilar products would help thousands more patients receive treatment with the money spent at present on reference biologics. Establishment of a rational policy on biosimilar substitution on institutional as well as national levels is recommended.

Disclosure of Interest: None Declared.

PEC007: Implementation of biosimilar uptake: a cost minimization study for insulin glargine

M. Mehring ${ }^{1}$ *, A. Heim ${ }^{1}$, C. Peyrol' ${ }^{1}$, A. Pages ${ }^{1}$, A. Bouothmani ${ }^{1}$, C. McCambridge ${ }^{1}$, P. Cestac ${ }^{1}$, A.-S. Mangé ${ }^{1}$

${ }^{1}$ Pharmacy, Toulouse, France

Background and objective: New french national recommendations aiming to optimize healthcare expenses were recently published. Prescribing biosimilars could be a strategic approach to minimize pharmaceutical costs in hospitals and in community pharmacies. In order to encourage physicians to prescribe biosimilar, hospitals will receive a remuneration depending on the biosimilar prescribing rate. Insulin glargine is one of the two target groups of biosimilar. The aim was to assess the prescribing trends and costs for both insulin glargine reference product (IRP) and biosimilar. Then we determined the potential annual savings of swiching to insulin glargine biosimilar (IB).

Design: Data was retrospectively collected over a 1-year period (January 2017 to December 2017) from our inventory management software. A minimizing cost study was conducted to estimate savings which could be made by prescribing biosimilar. The economic savings were quantified from insulin glargine consumption and per-unit price of IRP and IB.

Results: The total consumption of insulin glargine represented 4983 prescriptions comprising 276 biosimilar prescribing (5.5\%). Among 4707 IRP prescriptions, $4315(91.7 \%)$ could be potentially interchangeable without any dose adjustment, including 3407 IRP pen prescriptions ( $1.9 €$ for $300 \mathrm{UI}$ per pen) and 908 IRP vial prescriptions ( $25 €$ for 1000 UI per vial). The cost of IRP prescriptions rose to $29,173 €$. The interchangeability cost for IB could amount to $965 €$ ( $0.15 €$ for 300 UI per pen) saving $28,208 €$ in switching IRP for IB. Conclusion: The uptake of insulin glargine biosimilar has not been implemented whereas the hypothetical annual saving is significant. Contrary to generic drugs, the interchangeability for biosimilar remains a medical decision. A lack of information about biosimilar interchangeability could be a barrier to their use. In order to implement biosimilar prescribing, a pilot survey will be led in the geriatric unit care. Pharmacists will play a key role in supporting clinicians in making the decision to use a biosimilar. They will also be directly involved in patient education informing them about the decision to switch. A follow-up study will be undertaken after 3 months with the general practitioner to make sure that the switch is sustained. In the case of positive outcomes, the biosimilar interchangeability will be expanded to others wards to achieve maximum financial benefits.

Disclosure of Interest: None Declared.

\section{PT009: Rationality of parenteral proton pump inhibitors use} in Latvian hospital

A. Aitullina ${ }^{1, *}$, A. Prilina ${ }^{2}$, S. Purvina ${ }^{1}$

${ }^{1}$ Pharmacology, Riga Stradins University, ${ }^{2}$ Hospital pharmacy, Paul Stradins Clinical University hospital, Riga, Latvia

Background and objective: Overuse of Proton Pump Inhibitors (PPI) is well described problem in many clinical practices. ${ }^{1}$ Several studies showed that $27-81 \%$ of hospitalised patients receive PPI therapy without appropriate indication. ${ }^{2}$ Also inappropriate choice of 
intravenous (IV) route of administration could be classified as nonrational use.

Setting and method: Adult patients hospitalised in $02-03.2017$ to Pauls Stradins Clinical University hospital (PSCUH) surgery or internal medicine departments and received IV PPI therapy were included in this study. Information about patients' demographics, IV PPI use patterns and diagnoses was collected retrospectively from medical notes.

Main outcome measures: Indications for PPI use and ability to use drugs per os (e.g. parallel use of other drugs per os).

Results: 47 cases were included. 20 patients were men. Mean age was 66 years (24-91), mean duration of hospitalisation was 7 days (1-16) and mean PPI vials per case was 8 (2-30). 14 cases (30\%) are classified as rational IV PPI use, from them in 4 cases there was specific indication for IV PPI use, e.g. recurrent bleeding prophylaxis from upper gastrointestinal tract. In 10 cases PPI therapy was appropriate and there were not indicators that patients were able to use drugs per os. Mostly cases of rational IV PPI use were collected from gastroenterology department. Other 33 cases $(70 \%)$ are classified as nonrational IV PPI use. In 26 cases there were no clear indication for PPI use. In 20 cases patients were able to use PPI per os as they have received other drugs or food per os or via nasogastral tube. Costs of these 20 cases were 1064.65 EUR (199 vials). Alternative costs if instead of IV PPI patients would receive peroral PPI are 3.98 EUR/ 199 Omperasole capsules.

Conclusion: There is potential for an IV to PO switch project implementation in hospital surgery and internal medicine departments with an aim to reduce inappropriate use of IV PPI and consequently to decrease the costs of therapy.

\section{References}

1. Ital J Med. 2015;9(3):217-228; 2. Eur J Intern Med. 2016.; 3. Dig Dis Sci. 2009;54(9):1947-1952.

Disclosure of Interest: None Declared.

PT013: Safety and efficacy of pemetrexed as monotherapy for the maintenance treatment of metastatic non-small cell lung cancer

C. Aparicio Rubio ${ }^{1}{ }^{1 *}$, S. Cornejo Uixeda ${ }^{1}$, M. Prieto Castelló ${ }^{1}$, E. Monteagudo Santolaya ${ }^{1}$, G. Antonino de la Cámara ${ }^{1}$, B. Quintana Vergara ${ }^{1}$, A. Sánchez Alcaraz ${ }^{1}$

${ }^{1}$ Pharmacy, Hospital Universitario de la Ribera, Alzira, Spain

Background and objective: Non-small cell lung cancer (NSCLC) is a slow-growing cancer that begins with metastases in 50\% of cases. Pemetrexed is a multi-targeted anti-cancer antifolate agent that exerts its action by disrupting crucial folate-dependent metabolic processes essential for cell replication.

The objective is to evaluate safety and efficacy of Pemetrexed as monotherapy for the maintenance treatment of metastatic NSCLC (mNSCLC) and compare the results with JMEN-trial.

Setting and method: Retrospective study that includes diagnosed patients with mNSCLC and for which the treatment with pemetrexed as monotherapy for the maintenance has been evaluated $\left(500 \mathrm{mg} / \mathrm{m}^{2}\right.$ administered on the first day of each 21-day cycle), initiated from December 2012 to January 2017.

Main outcome measures: The variables were: age, sex, previous treatments, adverse effect (AE), Performance Status (PS), localization of metastases, progression-free survival (PFS) and overall survival (OS).

Data were compared with the results of JMEN-trial for mNSCLC (PFS median 4 months and OS 13.4 months).
Data have been obtained from the Electronic-Health-Record $\left(\right.$ SIAS $\left.^{\circledR}\right)$ and dispensation module $\left(\right.$ Farmis $\left.{ }^{\circledR}\right)$.

Results: The treatment has been requested for 24 patients, 6 were exitus before starting and 18 began the treatment.

The distribution of patients starting treatment was: 7 women and 11 men, average age 60 years. $45 \%$ had 0 PS and 55\% 1 PS. The main location of metastases were bone, hepatic and adrenal.

All patients who started treatment had previously received another line of therapy. The main treatments received were carboplatingemcitabine and cisplatin-gemcitabine.

During treatment all patients had AE. 39\% of these patients had fatigue, $33 \%$ anorexia, 28\% pharyngitis and 22\% diarrhoea and vomiting. $11 \%$ were admitted for febrile neutropenia and epileptic seizure.

At the time of the study, treatment was suspended to $89 \%$ of patients. The cause of discontinuation for these patients was due to progression of the disease. $87 \%$ of patients were exitus.

The PFS median was 4.5 months (95\% CI 0.9-34.9) and the OS median was 15.9 months (95\% CI 3.2-38.9).

Conclusion: Our results, compared with JMEN-trial, show better results in terms of PFS and OS than the one obtained in this study, but we must take into account the limited sample size $(n=18)$. Regarding the safety profile reactions described in the data sheet as very common were noted.

Disclosure of Interest: None Declared.

PT014: Safety and efficacy of trifluridine/tipiracil monotherapy in metastatic colorectal cancer

C. Aparicio Rubio ${ }^{1, *}$, E. Monteagudo Santolaya ${ }^{1}$, S. Cornejo Uixeda ${ }^{1}$, M. Prieto Castelló ${ }^{1}$,

G. Antonino de la Cámara ${ }^{1}$, B. Quintana Vergara ${ }^{1}$, A. Sánchez Alcaraz ${ }^{1}$

${ }^{1}$ Pharmacy, Hospital Universitario de la Ribera, Alzira, Spain

Background and objective: The combination drug trifluridine/tipiracil (TAS-102) is a novel oral nucleoside antitumor indicated for the treatment of adult patients with metastatic colorectal cancer (mCRC) who have been previously treated, or are not considered candidates for treatment, with available therapies.

The objective was to evaluate safety and efficacy of TAS-102 as monotherapy in $\mathrm{mCRC}$ and compare the results with RECOURSE-trial. Setting and method: Retrospective study that includes diagnosed patients with mCRC and for which the treatment with TAS-102 as monotherapy has been evaluated (starting dose $35 \mathrm{mg} / \mathrm{m}^{2} /$ dose administered orally twice daily on Days $1-5$ and Days $8-12$ of each 28-day cycle), initiated from January 2017 to January 2018.

Main outcome measures: The variables were: age, sex, previous treatments, adverse effect (AE), KRAS, Karnofsky Index (KI), progression-free survival (PFS) and overall survival (OS).

Data were compared with the results of RECOURSE-trial for mCRC (PFS median 2 months and OS 7.1 months).

Data have been obtained from the Electronic-Health-Record $\left(\right.$ SIAS $\left.^{\circledR}\right)$ and dispensation module (Abucasis ${ }^{\circledR}$ ).

Results: The treatment has been requested for 19 patients, 3 were exitus before starting and 16 began the treatment.

The distribution of patients starting treatment was: 6 women and 10 men, average age 71 years. $83 \%$ had KRAS-mutated and 17\% KRAS wild-type. KI median was $90 \%$ (95\% CI $80-100 \%$ ).

All patients who started treatment had previously received another line of therapy. The main treatments received were folfox-bevacizumab, folfiri-bevacizumab and panitumumab.

During treatment all patients had AE. $67 \%$ of these patients had malaise, 50\% anorexia, neutropenia and leukopenia, and 33\% 
anaemia and abdominal pain. $17 \%$ were admitted for bowel obstruction and renal failure.

These AE caused a reduction of $15 \%$ of the dose in $50 \%$ of patients. At the time of the study, treatment was suspended to $100 \%$ of patients. The cause of discontinuation for these patients was due to progression of the disease.

The PFS median was 1.27 months (95\% CI 0.67-4.8) and the OS median was 5 months (95\% CI 2.8-7.3).

Conclusion: Our results, compared with the RECOURSE trial, show lower results in terms of PFS and OS than the one obtained in this study, but we must take into account the limited sample size $(n=16)$. Regarding the safety profile reactions described in the data sheet as very common, common and uncommon occurred.

Disclosure of Interest: None Declared.

\section{PT015: Analysis of the influence of medication on fractures}

\section{Balbona-Bayona ${ }^{1, *}$, R. Madridejos ${ }^{2}$, P. Modamio ${ }^{1}$,}

\section{E. L. Mariño ${ }^{1}$}

${ }^{1}$ Clinical Pharmacy and Pharmacotherapy Unit, Department of Pharmacy and Pharmaceutical Technology, and Physical Chemistry, Faculty of Pharmacy and Food Sciences, University of Barcelona,

${ }^{2}$ Primary Health Care Unit, Mútua de Terrassa, Barcelona, Spain

Background and objective: Fractures represent an important health problem that have been increasing in recent decades. Falls are directly related to the incidence of fracture as well as age and sex. Besides, many studies have showed that commonly prescribed medication in daily practice were connected with an increase in the risk of fractures, either increasing the risk of falls or contributing to the loss of bone mineral density (BMD). The objective of the study was to analyse the incidence of fracture in patients over 60 years of age and to assess if there were any differences between the fractured and non-fractured population.

Setting and method: The present work was focused in eight basic health areas in the field of Primary Health Care. Two types of studies were performed, first a descriptive and transversal study of the fractured population during 2016 and another case-control study of the patients. Data was collected from the distribution program and clinical record of the patients. Statistical descriptive analysis of data was made.

Main outcome measures: Type of fracture, diagnosis, medication prescribed during 2016 and classified with ATC code.

Results: A total of 2527 patients were diagnosed with fracture (average age: $76.3 \pm 9.6 ; 76.8 \%$ women). The pharmacological register of 2300 patients (average age: $77.7 \pm 9.5 ; 76.8 \%$ women) was obtained, with an average prescription of $10.39 \pm 5.9$ medicines for each. $76.8 \%$ of the patients included in this study consumed at some point medications that decrease BMD, another $66.3 \%$ medications that increase the risk of falling and finally $85.6 \%$ used medications included in the two previous groups.

On the other hand, 4000 controls (average age: $73.9 \pm 9.1 ; 75.0 \%$ women), with an average prescription of $7.6 \pm 5.2$ medications/year were selected. $60 \%$ of them were using a medication that could decrease BMD, $46.7 \%$ medication that could induce falling and $72.8 \%$ a treatment included in both groups. In all cases, women had higher percentages.

Conclusion: A relevant association between the medication in the study and the risk of fracture was found. Due to the high prevalence of polymedicated patients, with probably one or more potentially relevant prescription medication, it would be highly recommendable to review the prescription of psychotropic drugs in order to reduce the risk of suffering a fracture.

Disclosure of Interest: None Declared.
PT016: PCSK9 inhibitors: differences between diabetic and nondiabetic patients

D. Blanquez Martinez ${ }^{1}$, M. Hayon Ponce ${ }^{2}$, I. Casas Hidalgo', X. Diaz Villamarin', R. Alvarez Sanchez ${ }^{1, *}$, P. Nieto Gomez, C. Davila Fajardo ${ }^{1}$

${ }^{1}$ Farmacia Hospitalaria, ${ }^{2}$ Endocrinologia y Nutricion, Hospital Universitario Campus de la Salud, Granada, Spain

Background and objective: Dyslipidemia represents the crucial link between diabetes and cardiovascular disease (CVD). Reduction of low-density lipoprotein cholesterol (LDL-c) is the most important strategy to prevent cardiovascular disease. In this context the newly developed proprotein convertase subtilisin kexin 9 (PCSK9) inhibitors are of great interest as they reduce LDL cholesterol by $50-70 \%$. To assess efficacy and safety of Alirocumab (ALI) and Evolocumab (EVO) in diabetic and non-diabetic patients with suboptimal LDL-c target values.

Setting and method: All patients with suboptimal LDL-c levels by maximal tolerated cholesterol lowering therapy were evaluated. Demographic data (age and sex), medical service prescribing, clinical data (CVD, cardiovascular risk factors (CVRF), presence of HFH and lipid-lowering treatment at baseline and during follow-up were recorded, biochemicals (total cholesterol (TC), LDL-c, HDL cholesterol (HDL-c), triglycerides (TG) and glycemia (GLY)) at baseline and after a treatment period of at least 3 months were collected. Response to PCSK9 between diabetic and non-diabetic patients was evaluated.

Main outcome measures: Total cholesterol, LDL cholesterol, HDL cholesterol, triglycerides and glycemia at baseline and after a treatment period of at least.

Results: 49 patients (63.3\% males). Mean age $54.7 \pm 10.7$ years. In the diabetic group $(\mathrm{n}=24), 38.5 \%$ Alirocumab $75 \mathrm{mg}$ and $61.5 \%$ Evolucumab 140 mg. 38.5\% statins intolerance, 53.8\% HFH. $76.9 \%$ CVD: $46.2 \%$ myocardial infarction, $23.1 \%$ arteriopathy, $7.7 \%$ stroke. A significant reduction in total cholesterol (TC) levels $112.3 \pm 66.5 \mathrm{mg} / \mathrm{dl}$ less $(p=0.004)$, LDL-c $111.6 \pm 65.9 \mathrm{mg} / \mathrm{dl}$ less $(p=0.004)(38.8 \% \mathrm{cLDL}<70)$ and TG $87.6 \pm 55.8 \mathrm{mg} / \mathrm{dl}$ less ( $p=0.006)$ was observed.

In the non-diabetic group $(\mathrm{n}=25), 48 \%$ Alirocumab $75 \mathrm{mg}$ and $52 \%$ Evolucumab $140 \mathrm{mg}$. 28\% had intolerance to statins. $32 \% \mathrm{HFH}$. 76\% CVD: $56 \%$ myocardial infarction, $16 \%$ arteriopathy, $4 \%$ stroke + myocardial infarction. This group also presented a significant reduction in CT levels $107.7 \pm 63.4 \mathrm{mg} / \mathrm{dl}$ less $(p<0.001)$, LDL-C $105.1 \pm 53.9 \mathrm{mg} / \mathrm{dl}$ less $(p<0.001)(32 \% \mathrm{cLDL}<70)$ and TG $78.1 \pm 53.3 \mathrm{mg} / \mathrm{dl}$ less $(p=0.025)$.

There were no significant differences in the results obtained between diabetics and non-diabetic patients. No significant increase in basal glycemia or glycosylated hemoglobin levels was observed in either group.

Conclusion: PCSK9 inhibitors in combination with statins, are one of the most promising and effective approaches to achieving very low LDL-c levels and reducing the risk of cardiovascular events. Diabetic patients show a similar response to PCSK9 inhibitors as non-diabetic patients.

Disclosure of Interest: None Declared.

PT017: Effectiveness and Safety of treatment with brentuximab vedotin

I. Casas Hidalgo ${ }^{1}$, P. Rodriguez Gomez ${ }^{1}$, D. Blanquez Martinez ${ }^{1}$, R. Alvarez Sanchez ${ }^{1, *}$

${ }^{1}$ Farmacia Hospitalaria, Hospital Universitario San Cecilio, Granada, Spain 
Background and objective: Background Brentuximab vedotine (BV) is a conjugated antibody formed by a monoclonal antibody directed against CD30.

Objective: The aim of this abstract is to describe the effectiveness and safety of brentuximab vedotin according to the experience of its use in clinical practice.

Design: Retrospective observational study from January 2015 to March 2017 of all patients who started treatment with BV in a tertiary level hospital. The variables analyzed were sex, age, diagnosis, stage of the disease, previous treatments, number of cycles of BV administered, transplant afterwards, effectiveness according to Cheson criteria (confirmed by PET) and adverse effects.

Results: Twelve patients were included. $58 \%$ of the patients were men and the median age was 42 years (range 22-67). Diagnosis: nodular-sclerosis classical hodgkin lymphoma (10 patients), anaplastic large-cell lymphoma (1 patient) and mycosis fungoides transformed into large T-cell lymphoma (1 patient). Stage of disease: I (1/12), II (6/12), III (1/12), IV (4/12). Five patients were given BV when their disease was refractory to other treatments and 7 patients were relapsed. The median of previous cycles was 10 (range 14-7). The most frequent previous treatments were: ABVD (10/12), ESHAPMINE (8/12), CHOP (5/12), GPD (5/12). The median of BV cycles received was 4 (range 1-7). In 5 patients (all of whom were diagnosed with Nodular-Sclerosis Classical Hodgkin Lymphoma) a subsequent transplant was performed: TASPE (2/5) and aloTPH (5/5). Effectiveness: Complete remission (CR) (4/12), partial remission (PR) (5/ 12), Progressive disease (PD) (2/12) and pending evaluation (1/12). Adverse effects were manifested in $33.33 \%$ of patients (abdominal discomfort, erythematous lesions, grade II (reversible) peripheral sensory neuropathy and infusional reaction despite premedication. 2 patients die short time after starting treatment with BV.

Conclusion: Brentuximab vedotin is an effective treatment in patients with nodular-sclerosis classical hodgkin lymphoma, leading to a complete or partial remission in the majority of patients undergoing such treatment. However, this drug caused toxicity that led to the withdrawal of this treatment in one of the patients. However, longerterm, population-based studies are needed to identify the benefit-risk balance as well as to identify population subgroups most likely to respond with the lowest rate of adverse effects.

Disclosure of Interest: None Declared.

\section{PT018: Probiotics for the treatment of necrotizing enterocolitis} in neonates

I. Casas Hidalgo ${ }^{1}$, M. Valle Corpas ${ }^{1}$, M. D. L. P. Casas Hidalgo ${ }^{2}$, R. Alvarez Sanchez ${ }^{1, *}$

${ }^{1}$ Farmacia Hospitalaria, ${ }^{2}$ Microbiologia y Parasitología, Hospital Universitario San Cecilio, Granada, Spain

Background and objective: Necrotizing enterocolitis (NEC) is the most frequent cause of acquired gastrointestinal disease and surgical emergency in the neonate. It affects predominantly preterm infants with very low birth weight $(10 \%)$. The pathogenesis of NEC is multifactorial and although it is not clearly defined, one has the theory that the integrity of the gastrointestinal tract is compromised as a result of the interaction of intestinal immaturity, microbiota alteration, enteral feeding and ischemia. Infants affected by this pathology may have short- and long-term complications and sequelae, such as sepsis, cholestasis, extrauterine malnutrition, short bowel syndrome, and altered growth and neurodevelopment (35\%). Mortality is high $(20-50 \%)$. The preventive measures used, including the administration of probiotics, have shown a great impact on the incidence and morbidity of the NEC.

The aim of this study is to determine the efficacy and proper management of probiotics for the prevention of NEC in preterm infants.
Design: In order to find the maximum number of studies associating the use of probiotics with the prevention of NEC, a thorough search was conducted in MEDLINE, the Central Register of Controlled Trials and EMBASE. The MeSH descriptors used were "neonate", "infant", "newborn", "probiotic" and "necrotizing enterocolitis". Studies carried out between January 1980 and March 2017 were considered.

Results: The use of probiotics should be strongly considered in the care of premature $<32$ weeks of gestation and/or $<1500 \mathrm{~g}$.

- Although the benefit-risk-cost balance is clearly favorable with current data, the routine use of probiotics would require close monitoring.

Based on the randomized clinical trials performed to date, it is possible to give the following recommendations as to type of strain, dose, start and duration:

- What strain?: A combination of Bifidobacterium and Lactobacillus is preferable.

- What dose: $3 \times 109 \mathrm{CFU} /$ day, preferable in single dose. In < $1000 \mathrm{~g}$ start with $1.5 \times 108 \mathrm{CFU} /$ day until reaching enteral of $50 \mathrm{ml} / \mathrm{kg} /$ day.

- When to start: when you can start enteral feeding, preferable in the first 7 days of life.

- How long to continue?: up to 35 weeks of postconceptional age or until discharge.

- Any precautions?: Do not offer the supplement with probiotics if acute illness such as sepsis, NEC or perinatal asphyxia.

Conclusion: After reading the various meta-analyzes and clinical trials, it can be stated that probiotics may lead to an improvement in NEC, although it is important to note that there are few available studies related to the safety and effect of probiotics in preterm infants with extreme low birth weight $(<1000 \mathrm{~g})$, as well as economic studies to determine their cost-effectiveness in health systems. Consequently, the most reasonable thing is to have a cautious attitude to the introduction of probiotics in the preterm in order to determine better the safety and to evaluate the effects in the long term.

Disclosure of Interest: None Declared.

\section{PT019: Benznidazol: clinical practice in a tertiary hospital}

\section{Casas Hidalgo ${ }^{1}$, P. Nieto Gómez ${ }^{1}$, D. Blanquez Martinez ${ }^{1}$,} R. Alvarez Sanchez ${ }^{1, *}$

${ }^{1}$ Farmacia Hospitalaria, Hospital Universitario San Cecilio, Granada, Spain

Background and objective: Trypanosoma cruzi, which causes Chagas' disease, is one of the most common parasites in tropical areas. Nowadays, the treatment of such parasite is based on Benznidazol. However, Benznidazol is not commercialized in Spain. The treatment for chronic Chagas' disease is highly controversial because of the difficulties involved in assessing its therapeutic efficacy and the different side effects of the treatment. Hospital pharmacists are responsible for applying this treatment to patients, only after the elaboration of an exhaustive report. In this article, we have reviewed all the reports as well as classified the information in order to improve the knowledge of the clinical practice in our hospital.

The purpose of this study is to improve the understanding of the clinical practice regarding the treatment of the Trypanosoma cruzi.

Design: Observational descriptive study from January 2013 to September 2016. Dates of patients were obtained from their clinical history. Examined variables: age, sex, nationality, symptomatology of the disease, coinfections, doses and side effects of Benznidazol. 
Results: A total of 97 patients were analysed-41 men and 56 women. The average age was $37.27 \pm 7.73$. Nationality: all the patients were from Bolivia. Only $41.24 \%$ of the patients had some symptomatology of the disease (constipation, dyspnoea, fatigue, cardiomyopathy). Coinfections: 42 patients (43.29\%): strongyloidosis, syphilis, toxocariasis, tuberculosis, BHV, intestinal amebiasis, uncinariasis, himinolepis and schistosomiasis. Posology: $7 \mathrm{mg} / \mathrm{kg} / \mathrm{day}$ during 60 days. Side effects: 48 patients (49.48\%): injuries cutaneous (itchy skin lesions (36 patients), headache (17 patients), gastrointestinal symptoms (epigastric pain, anorexia, nausea, pyrosis, diarrhea) (5 patients), distal neuropathy ( 3 patients), dizziness ( 3 patients), edema of lips (3 patients), muscle pain ( 2 patients), cramps ( 2 patients), weight loss (1 patient). Three patients stopped taking the drug and five patients had to decrease the dose of Benznidazol because of the appearance of such a serious adverse effects.

Conclusion: Benznidazole is an etiological treatment commercially available for the Chagas' disease. However, use of this drug is associated with a wide range of side effects which could lead to patients stopping taking it. Therefore, pharmacists could play an important role in both recognizing the adverse effects and proposing an individualized dose adjustment of Benznidazol for each patient in order to avoid treatment discontinuation and prevent the development of cardiomyopathy and transmission by blood transfusion, congenital and organ transplants of this disease.

Disclosure of Interest: None Declared.

PT020: Immunotherapy vs BRAF and MEK inhibitors in the treatment of metastatic melanoma

\section{Casas Hidalgo ${ }^{1}$, P. Nieto Gómez ${ }^{1}$, A. Rodriguez Delgado ${ }^{1}$, \\ R. Alvarez Sanchez,**}

${ }^{1}$ Farmacia Hospitalaria, Hospital Universitario San Cecilio, Granada, Spain

Background and objective: Background The treatment of metastatic melanoma is almost always incurable with a median survival of 6-9 months, a $25 \%$ survival per year and $15 \%$ survival at 3 years. This situation has changed radically since a much greater survival has been demonstrated with the use of two therapeutic approaches: immunomodulation (Ipilimumab, Nivolumab, Prembrolizumab) and drugs against target mutations in tumor cells (BRAF inhibitors: Vemurafenib and Dabrafenib; and MEK inhibitors: Trametinib and Cobimetinib).

Objective: To evaluate the baseline characteristics of the patients as well as the use of this type of therapy in a secondary hospital.

Design: Retrospective observational study from January 2016 to March 2018 of all patients treated with inmunotherapy, BRAF inhibitors and/or MEK inhibitors. The variables analyzed were:

- Baseline characteristics of patients: sex, age.

- Features related to pharmacological treatment: posology, line therapy, number of cycles administered, monotherapy/bitherapy.

Results: Number of patients: Nivolumab (NIVO) 5 patients; Pembrolizumab (PEMBRO) 3 patients; Vemurafenib (VEMURA) 8 patients;Dabrafenib (DABRA) 14 patients;Trametinib (TRAME) 16 patients and Cobimetinib (COBI) 4 patients.

* sex (\% patients): NIVO 60\% men, PEMBRO 50\% men, VEMURA $75 \%$ men, DABRA $64 \%$ women, TRAME $72 \%$ women, COBI $50 \%$ men.

* age (mean $\pm \mathrm{sd}$ ): NIVO $57.6 \pm 9.79$; PEMBRO $54 \pm 4.95$; VEMURA $58 \pm 9.84$; DABRA $60 \pm 15.47$; TRAME $63 \pm 16.21$; COBI $59 \pm 2.67$.

* dosage: NIVO $3 \mathrm{mg} / \mathrm{kg}$ every 2 weeks, PEMBRO $2 \mathrm{mg} / \mathrm{kg}$ every

3 weeks, VEMURA $960 \mathrm{mg}$ every $12 \mathrm{~h}$, DABRA $150 \mathrm{mg}$ every
$12 \mathrm{~h}$, TRAME $2 \mathrm{mg}$ every $24 \mathrm{~h}$, COBI $60 \mathrm{mg}$ day $1-21$ every 28 days.

* most frequent line of treatment (\% patients): NIVO 2nd line (60\%), PEMBRO 2nd line (100\%), VEMURA 1st line (50\%), DABRA 2nd line (57\%), TRAME 2nd line (50\%), COBI 1st line $(50 \%)$.

* number of cycles administered (mean \pm sd): NIVO $14 \pm 8.54$; PEMBRO $7 \pm 7.07$; VEMURA $19 \pm 11.36$; DABRA $23 \pm 9.85$; TRAME $32 \pm 17.89$; COBI $11 \pm 11.15$.

* monotherapy vs bitherapy (\% patients): NIVO 100\% monotherapy; PEMBRO $100 \%$ monotherapy; VEMURA $50 \%$ bitherapy (+ Cobimetinib), 50\% monotherapy;DABRA 100\% bitherapy (+ Trametinib), TRAME $85 \%$ bitherapy (+ Dabrafenib), $15 \%$ monotherapy; COBI $100 \%$ bitherapy (+ Vemurafenib).

Conclusion: According to this study, immunomodulation is always used in monotherapy; whereas BRAF and MEK inhibitors are usually used in combination (bitherapy). In addition, the order of the use (line therapy) of the new different drugs available for the treatment of metastatic melanoma is not clear, therefore more robust studies are needed to determine it.

Disclosure of Interest: None Declared.

PT021: FLOT perioperative chemotherapy in patients with locally advanced gastric adenocarcinoma: evaluation of safety and tolerability

K. Chetouane ${ }^{1, *}$, A.-S. Plichet ${ }^{1}$, E. Ducret ${ }^{1}$, I. Trouilloud $^{2}$, C. Fernandez ${ }^{1}$, A.-C. Joly ${ }^{1}$, M.-T. Baylatry ${ }^{1}$

${ }^{1}$ Pharmacy, ${ }^{2}$ Oncology, Saint-Antoine Hospital, APHP, Paris, France

Background and objective: FLOT regimen has been recently considered as the new standard of care in perioperative treatment of patients with gastric adenocarcinoma (median overall survival increasing from 35 to 50 months $(p=0.012)$, FLOT4, phase 3 clinical trial, ASCO June 2017). It consists of 4 pre-and 4 post-operative cycles of infusional 5 -Fluorouracile $(5-\mathrm{FU}) 2600 \mathrm{mg} / \mathrm{m}^{2}$ over $24 \mathrm{~h}$, leucoverin $200 \mathrm{mg} / \mathrm{m}^{2}$, oxaliplatin $85 \mathrm{mg} / \mathrm{m}^{2}$ and docetaxel $50 \mathrm{mg} /$ $\mathrm{m}^{2}$, every 2 weeks.

The aim of this study was to evaluate safety and tolerability of perioperative chemotherapy FLOT, in operable patients with gastric adenocarcinoma in our clinical practice.

Setting and method: Retrospective observational hospital study in Oncology department: operable patients with locally advanced gastric adenocarcinoma were treated with FLOT from June 2017 to April 2018.

Data were collected from CHIMIO ${ }^{\circledR}$ and ORBIS ${ }^{\circledR}$ hospital softwares.

Adverse events were recorded and graded according to NCI-CTC classification.

Main outcome measures: Patients' characteristics: age, sex, performance status (PS), number of chemotherapy cycles. Tolerability: occurrence of adverse events.

Results: 7 patients (5 men, 2 women, mean age of 60 years [45, 78], PS 0-1) were included.

No patient completed all eight cycles of perioperative chemotherapy.

5 patients $(71 \%)$ completed 4 cycles of preoperative FLOT and proceeded to surgery.

Only one of them started postoperative chemotherapy and received 3 cycles of postoperative FLOT (including 2 cycles without docetaxel because of side effects).

Multiple adverse events were observed: $94 \%$ of them were $\leq$ grade3 (nausea, diarrhea, neuropathy...),1 patient had neutropenia grade 4 and 1 patient died 10 days after the first cycle of chemotherapy FLOT due to mesenteric ischemia. 
Conclusion: Due to tolerability issues in our hospital, FLOT has been completely stopped with no further new inclusions. Patients were switched to FOLFOX perioperative chemotherapy (infusional 5-FU $1200 \mathrm{mg} / \mathrm{m}^{2}$ over $46 \mathrm{~h}$, bolus 5 -FU $400 \mathrm{mg} / \mathrm{m}^{2}$, oxaliplatin $85 \mathrm{mg} /$ $\mathrm{m}^{2}$ ), a protocol previously used as a treatment of local gastric adenocarcinoma

Two other patients treated with FLOT in French hospitals died due to mesenteric ischemia (Pharmacovigilance case reports). This toxicity is probably attributed to docetaxel -5-FU combined therapy.

FLOT regimen is a promising perioperative chemotherapy in clinical trials but in clinical practice, side effects and morbidity should be carefully considered and patients need to be selected.

Disclosure of Interest: None Declared.

PT022: Multidrug resistant Pseudomonas aeruginosa infection in a lung transplant recipient with cystic fibrosis: which doses for ceftazidime/avibactam?

C. Faure ${ }^{1, *}$, H. Pluchart ${ }^{1,2,3}$, L. Quarteroni ${ }^{1}$, S. Quetant ${ }^{4}$, J. Claustre ${ }^{4,5}$, S. Chanoine ${ }^{1,2}$, P. Bedouch ${ }^{1,2,3}$

${ }^{1}$ Pharmacy Department, Grenoble Alpes University Hospital, ${ }^{2}$ School of pharmacy, ${ }^{3} \mathrm{CNRS}$, TIMC-IMAG UMR5525/ThEMAS, Grenoble Alpes University, ${ }^{4}$ Pneumology Unit, Grenoble Alpes University Hospital, ${ }^{5}$ School of medicine, Grenoble Alpes University, Grenoble, France

Background and objective: Chronic Pseudomonas aeruginosa (PA) colonization in lung transplant recipients with cystic fibrosis (CF) is a complex situation with difficult therapeutic management. There is a lack of information about Ceftazidime/avibactam (CEF/AVI) efficacy in patients with $\mathrm{CF}$ and about drug-drug interaction. We report the first CEF/AVI use in multidrug resistant (MDR) PA infection in patient lung transplant recipient with $\mathrm{CF}$.

Design: Case report.

Results: A 31-year-old man with CF colonized by PA was lungtransplanted in August 2015. Chronic colonization by PA (sensitive to ceftazidime) occurred in October 2015 leading to introduce a daily therapy by Nebulized colistin to prevent acute exacerbations. His immunosuppressive therapy included tacrolimus, mycophenolate mofetil and corticosteroids. In December 2017, purulent sputum led to bacterial susceptibility test based on sputum analysis and showed MDR PA except for new associations of cephalosporins with betalactamase inhibitors, CEF/AVI and ceftolozane/tazobactam. Physicians decided to introduce CEF/AVI for which usual dose regimen is $2 \mathrm{~g} / 0.5 \mathrm{~g} 3$ times a day. However, in $\mathrm{CF}$, drugs pharmacokinetic changes, such as increased volume of distribution, hypothetic higher active tubular secretion, viscous bronchial secretions and biofilm result in high antibiotics doses. In literature, only one case reported successful use of CEF/AVI in a CF patient at usual regimen. So, physicians and pharmacists agreed to start with this dose. The onset of acute hives in toe during the first dose of CEF/AVI in relation to known hives with ceftazidime led to introduce cetirizine $10 \mathrm{mg}$ per day. Tacrolimus through concentrations were measured to determine potential drug-drug interaction with CEF/AVI. Clinical improvement and patient willingness to have home-care led to continue CEF/AVI at home for a total duration of CEF/AVI treatment of 14 days. Nebulized colistin was continued with CEF/AVI. Tacrolimus concentration was not altered by CEF/AVI therapy associated with clinical improvement 1 month later.

Conclusion: CEF/AVI is a new therapeutic option in the management of the PA colonized-CF patient. Despite a lack of data in the literature concerning its use, doses and pharmacokinetic parameters in $\mathrm{CF}$, clinical improvement with the usual dose regimen is showed even if in standard practices the dose of ceftazidime is doubled.

Disclosure of Interest: None Declared.
PT024: Use of biological therapy in the treatment of psoriasis

G. López-Llaó $^{1, *}$, N. Pola ${ }^{1,2}$, P. Modamio ${ }^{1}$, C. Muñoz ${ }^{3}$, N. Rudi ${ }^{1,2}$, E. L. Mariño ${ }^{1}$

${ }^{1}$ Clinical Pharmacy and Pharmacotherapy Unit, Department of Pharmacy and Pharmaceutical Technology, and Physical Chemistry, Faculty of Pharmacy and Food Sciences, University of Barcelona, Barcelona, ${ }^{2}$ Hospital Pharmacy Service, ${ }^{3}$ Dermatology Service, Hospital General de Granollers, Granollers, Barcelona, Spain

Background and objective: Psoriasis is a chronic and recurrent autoimmune disease characterised by a rapid build up of cells on the surface of the skin forming scales, inflammation and red patches. Although the etiology is yet unknown, it seems to have genetic causes and environmental factors such as stress, infections, alcohol consumption, tobacco or due to the use of certain medicines. Psoriasis treatments do not cure, but chase the remission of the disease or reduction of the lesions. The objective of this study was to evaluate the effectiveness and safety in clinical practice of biological medications used in patients suffering from psoriasis.

Setting and method: This work was split into two parts. First of all, a cross-sectional study was carried out in patients who attended the Dermatology Department of the hospital (date 01/10/2017) in order to know the age, sex, biological medication used and it's posology. Then, a descriptive and retrospective study was performed in a random sample of 27 patients. The clinical histories of the patients (since it's first implementation electronically until the present date of October 2017) were used to obtain the data.

Main outcome measures: The effectiveness, safety and persistence of the biological therapy.

Results: Patients included in this study were mostly men $(69.1 \%)$ with an average age between 41 and 60 years old. The biological drug most commonly used was ustekinumab, followed by secukinumab, which was normally used as a rescue medication. In $48.8 \%$ of cases, patients used a deintensification posology. When evaluating the effectiveness of the therapy; adalimumab, secukinumab and ustekinumab showed to be the most effective biological treatments for psoriasis. As for the adverse drug effects, most of them were related to infections, although these were not severe. Finally, when considering the medication compliance and persistence, in general, first biological selected showed to have the best persistence with $65 \%$ of patients still continuing the treatment at the end of the first year, unlike had a low $35 \%$ in treatment persistence at the end of the third year.

Conclusion: It is important to analyse the data obtained from the clinical practice in order to know the effectiveness and safety of biological therapy, with the aim of achieving a more personalised adjustment of the medication for each patient and therefore a higher treatment persistence, knowing the fact that biological therapy has a significant impact on health resources.

Disclosure of Interest: None Declared.

\section{PT025: Real-world effectiveness and safety of alirocumab}

C. Mondelo García ${ }^{1, *}$, M. García Queiruga ${ }^{1}$, J. Balea Filgueiras ${ }^{1}$, N. Fernández Bargiela ${ }^{1}$, V. Giménez Arufe ${ }^{1}$, C. Fernández Oliveira ${ }^{1}$, M. I. Martín Herranz ${ }^{1}$

${ }^{1}$ Pharmacy, Complexo Hospitalario Universitario a Coruña, A Coruña, Spain

Background and objective: Hypercholesterolemia is a growing health problem in developed countries which can cause serious consequencies in patients who suffer from it. Alirocumab is a Proprotein Convertase Subtilisin Kexin Type 9 (PCSK9) inhibitor antibody indicated as adjunct to diet and maximally tolerated statin therapy for 
the treatment of adults with heterozygous familial hypercholesterolemia or clinical atherosclerotic cardiovascular disease, who require additional lowering of LDL-cholesterol (c-LDL). Our objective is to analyze the effectiveness and safety of alirocumab in a tertiary hospital.

Design: Observational retrospective study of $100 \%$ treated with alirocumab from 1st December 2016 to 30th April 2018. Main variables: sex, age, cause of statins failure, previous clinical trial, alirocumab dose, adverse effects and LDL cholesterol levels after 3-months treatment. Data sources: electronic prescription program and electronic medical records.

Results: 70 patients included. Mean age: $60 \pm 11.6$ years old; $64 \%$ male. All patients included in this study were instructed in the correct use of the dispositive of alirocumab in the first visit to the Hospital Pharmacy. 43 patients $(61 \%)$ had to start treatment with alirocumab due to the ineffectiveness of statins and patients $(39 \%)$ had to start treatment with alirocumab because of statins intolerance (muscle pain) disappearing completely the muscle symptoms with the treatment change. 16 patients $(23 \%)$ were previously treated with anti PCSK9 in clinical trials. Depending on LDL cholesterol levels at the beginning of the treatment: 62 patients $(89 \%)$ receive alirocumab $75 \mathrm{mg}$ every 14 days, 5 patients $(7 \%)$ receive alirocumab $150 \mathrm{mg}$ every 14 days and 3 patients (4\%) receive alirocumab $150 \mathrm{mg}$ every 28 days. Patients previously included in clinical trials with anti PCSK9 continued with appropriate levels of LDL cholesterol and all patients who started alirocumab treatment during the study period achieved appropriate levels of c-LDL in 3-months treatment: $63.7 \pm 25 \mathrm{mg} / \mathrm{dL}$. Reported adverse effects were few and slight: rinitis (4 patients, one of them with epistaxis), diarrhea ( 2 patients), cutaneous reactions ( 3 patients) and jaw pain (1 patient).

Conclusion: Alirocumab is an effective, safe and well tolerated treatment to decrease c-LDL until appropriate levels are attained in patients who are intolerant to statins or when statins are ineffective. Disclosure of Interest: None Declared.

\section{PT026: Effectiveness and safety of secukinumab in patients} with moderate to severe plaque psoriasis

\section{R. Morón ${ }^{1}$, R. Alvarez ${ }^{1, *}$, P. Nieto ${ }^{1}$, C. García ${ }^{1}$, S. Belda ${ }^{1}$, J. Cabeza ${ }^{1}$ \\ ${ }^{1}$ Complejo Hospitalario Universitario de Granada, Complejo Hospitalario Universitario de Granada, Granada, Spain}

Background and objective: Secukinumab is a recently approved interleukin 17A inhibitor indicated for the treatment of patients with moderate to severe plaque psoriasis (MSPP), psoriatic arthritis and ankylosing spondylarthritis.

The aim of the study was to assess the effectiveness and safety of secukinumab in patients with MSPP.

Design: Retrospective study performed in a third-level hospital. Patients with MSPP who started treatment with secukinumab between January 2016 and April 2017 were included. Demographic, clinical and treatment variables (previous systemic therapies and other biological treatments) at baseline were collected. Effectiveness and safety were assessed based on the psoriasis area severity index (PASI) after 12, 24 and 52 weeks respectively. These data were obtained from medical records. The variables are presented by means and percentages.

Results: A total of 33 patients were selected, with a mean age of 47 years, of whom 17 (51\%) were males. All patients had MSPP. 26 (79\%) patients had received prior non-biological systemic treatment. 20 (60\%) patients had failed prior biologics. The most commonly used biologics were etanercept, adalimumab and ustekinumab. After 12 weeks of treatment $31(94 \%)$ patients had achieved a PASI 50, 29 $(88 \%)$ a PASI 75 and $25(76 \%)$ a PASI 100 . At week 24 of treatment 33
(100\%) patients had achieved a PASI 50, $33(100 \%)$ a PASI 75 and 31 (94\%) a PASI 100 . Finally at week 52 of treatment $31(93 \%)$ patients had achieved a PASI 50, 31 (93\%) a PASI 75 and $27(82 \%)$ a PASI 100. Regarding safety, no patient experienced any adverse reaction.

Conclusion: In this study, secukinumab shows high effectiveness, $76 \%$ patients achieving a PASI of 100 at week 12 both in naive and in previously treated patients. No differences were observed in PASI 100 both at week 24 and at week 52 compared to week 12 .

Secukinumab is well tolerated, with a good safety profile and without discontinuations due to adverse effects. Therefore, it can be considered a good therapeutic option in patients with moderate to severe psoriasis who are non-responders or who have a contraindication or intolerance to systemic treatments or phototherapy.

Disclosure of Interest: None Declared.

PT027: Foscarnet use in multidrug-resistant cytomegalovirus in lung-transplanted patient: is it a feasible option?

L. Quarteroni ${ }^{1, *}$, H. Pluchart ${ }^{1,2,3}$, C. Faure ${ }^{1}$, A. Briault ${ }^{4}$, S. Chanoine ${ }^{1,2}$, J. Claustre ${ }^{4,5}$, P. Bedouch ${ }^{1,2,3}$

${ }^{1}$ Pharmacy Department, Grenoble Alpes University Hospital, ${ }^{2}$ School of pharmacy, ${ }^{3}$ CNRS, TIMC-IMAG UMR5525/ThEMAS, Grenoble Alpes University, ${ }^{4}$ Pneumology Unit, Grenoble Alpes University Hospital, ${ }^{5}$ School of medicine, Grenoble Alpes University, Grenoble, France

Background and objective: Cytomegalovirus (CMV) infection is a potentially severe affection in lung transplant recipients. It is associated with an increased risk of CMV disease onset. If CMV infection occurs, first-line anti-CMV drugs are Valganciclovir (VGC) and ganciclovir (GCV). Foscarnet (FOS) and cidofovir (CFV) are used for resistant-CMV. In literature, few case reports relate both efficacy and safety of FOS in lung transplant recipients with multidrug-resistant (MDR) CMV. This case aimed at reporting FOS use in a lung transplant recipient with MDR CMV.

Design: Case report.

Results: A 67-year-old man was lung-transplanted in October 2016 with a CMV serological mismatch (seropositive donor and seronegative recipient), with an initial prophylaxis by VGC for 1 year. During VGC prophylaxis, he developed CMV primary infection in January 2017. He was treated by GCV $5 \mathrm{mg} / \mathrm{kg}$ twice a day (bid) during 1 month then by VGC (900 mg bid). After failure of oral therapy with VGC due to low through concentration (Cmin) at $0.8 \mathrm{mg} / \mathrm{L}$ with target concentration superior to $1.3 \mathrm{mg} / \mathrm{L}$, treatment by GCV $5 \mathrm{mg} / \mathrm{kg}$ bid was reintroduced. GCV Cmin was $0.8 \mathrm{mg} / \mathrm{L}$, dose was elevated to $10 \mathrm{mg} / \mathrm{kg}$ bid. Immunosuppressive treatment doses were reduced, polyvalent immunoglobulin and everolimus were initiated. F412L and D588 N/D mutations on UL54 CMV protein which conferred GCV, CFV and FOS resistance were detected. In theory GCV, CFV, FOS were not efficient. Despite these results, GCV was substituted by FOS $120 \mathrm{mg} / \mathrm{kg}$ bid because of persistent positive CMV viral load. Hematuria and tubulopathy were discovered and FOS dose was reduced to $120 \mathrm{mg} / \mathrm{kg}$ per day. FOS was continued during 3 months for treatment of the CMV infection and secondary prophylaxis, with maintenance of CMV negative viral load. Despite some mild ionic disorders, there was no renal insufficiency, a satisfactory clinical tolerance was observed.

Conclusion: This is the first case of FOS safety and efficacy in a lung transplant recipient with multidrug-resistant CMV. The success of CMV treatment is multifactorial: type of CMV-mutation, firstly immunosuppression reduction, and secondly everolimus introduction. Indeed, everolimus has a protective effect against CMV infection in solid organ transplants. Despite in vitro resistance, FOS led to biological suppression of CMV replication.

Disclosure of Interest: None Declared. 
PT028: Determination of patients' knowledge about Helicobacter pylori treatment and evaluate consistency of their Helicobacter pylori treatment regimen with the guidelines

\section{Y. Yesilbursa ${ }^{1}$, Y. Sahin ${ }^{1, *}$, B. Okuyan', F. V. Izzettin ${ }^{1}$, M. Sancar ${ }^{1}$ \\ ${ }^{1}$ Marmara University, Istanbul, Turkey}

Background and objective: To determine patients' knowledge regarding their Helicobacter pylori infection and its treatment and to evaluate consistency of their Helicobacter pylori treatment regimen with the guidelines.

Setting and method: This cross-sectional study was conducted in two different community pharmacies located in Istanbul, Turkey between September 2017 and January 2018. Information gathered within self-structured questionnaire.

Main outcome measures: Determining the patients' level of information about their disease and treatment by their responses on selfstructured questionnaire and evaluating consistency of their treatments with guidelines suggested therapies according to evidencebased antibiotic resistance data.

Results: Of 138 participant patients (95 female, mean age $47.06 \pm 13.86$ ), $31 \%$ have peptic ulcer history and $14.49 \%$ have family history of gastric cancer. Of them, $75 \%$ received Helicobacter pylori eradication treatment for the first time and $56 \%$ were regularly using a proton pump inhibitor before Helicobacter pylori treatment. Female patients more likely knew Helicobacter pylori as an infection when compared with male patients $(p=0.014)$. Patients who received the treatment more than once, more likely knew Helicobacter pylori as an infection when compared with the patients who received the treatment for the first time $(p=0.025)$. Patients with higher education level more likely knew Helicobacter pylori as an infection $(p=0.002)$. And patients with higher education level more likely knew that gastric ulcers would be occurred as a consequence of Helicobacter pylori $(p=0.018)$. Although Turkey is one of the countries that clarithromycin resistance is high (1), $75.4 \%$ of patients received standard triple therapy, which consisted clarithromycin in this study. According to guidelines, second-line therapy antibiotics should be different from first-line therapy. However, it was determined that antibiotics remained same as first line therapy in the $57.14 \%$ of the patients' second line therapy.

Conclusion: Based on insufficient knowledge level in most of patients, patient education regarding Helicobacter pylori treatment would make a difference on health-related outcomes. Helicobacter pylori treatment strategies should be updated according to evidencebased antibiotic resistance data to improve therapeutic outcomes.

\section{Reference}

1. Goktug Onder, MD, Ahmet Aydin, MD, Ulus Akarca, MD, Fatih Tekin, MD, Omer Ozutemiz, MD, and Tankut Ilter, MD. High Helicobacter pylori resistance rate to clarithromycin in Turkey. Journal of Clinical Gastroenterology, 2007 September; 41(8):747-750

Disclosure of Interest: None Declared.

PT031: Oxaliplatin desensitization procedure is safe and feasible in oncology day-hospital

E. Lepoix ${ }^{1}$, D. Botsen ${ }^{1}$, C. Mazza' ${ }^{1}$, M. Brasseur ${ }^{1}$, A. Grange ${ }^{2}$, F. Slimano ${ }^{3,4, *}$, O. Bouché ${ }^{1,5}$

${ }^{1}$ UMA-CH (Oncology-day Hospital), ${ }^{2}$ Department of dermatology, ${ }^{3}$ Department of Pharmacy, CHU Reims, ${ }^{4}$ Facutly of Pharmacy,

${ }^{5}$ Faculty of Medicine, Reims University, Reims, France
Background and objective: Oxaliplatin-based regimens are widely used in gastrointestinal oncology but oxaliplatin can cause Hypersensibility Reactions (HSR) during infusion and this lead to permanent treatment discontinuation. These HSR occurs from 0.5 to $25 \%$ depending on the recent treatment history with oxaliplatin. Desensitization procedures are used without standard but all need a complete hospitalization at least the day before. The feasibility of desensitization protocols is unknown in oncology-day hospital whereas it can enhance quality of life and cost minimization.

Setting and method: We retrospectively analysed results from patients treated in oncology-day hospital between January, 2016 and December, 2017 by an oxaliplatin desensitization protocol commonly used in complete hospitalization with $\mathrm{H}-48$ and $\mathrm{H}-24$ premedication (antihistaminergic). Patients had to be treat by at least two desensitization protocols before be re-treating by a conventional oxaliplatinbased regimen. Demographic data, number of infusions before HSR, HSR description and desensitization procedures were recorded and analysed regarding literature.

Main outcome measures: To assess the safety and feasibility of oxaliplatin desensitization procedures in an oncology-day hospital in terms of success rate compared to literature.

Results: During 2 years $n=427$ patients were treated by oxaliplatin and $n=22$ experienced HSR $(5.15 \%)$. Five patients were selected for desensitization (the mean number of infusion before HSR was 4.4 and HSR were grade $3(n=1)$ or less $(n=4)$. They were treated by 12 desensitization procedures in oncology-day hospital. All patients were compliant with $\mathrm{H}-48$ and $\mathrm{H}-24$ premedication at home. $\mathrm{N}=10 / 12$ infusions were successful (no immediate or delayed adverse events) consistently with literature. Three patients were subsequently retreated by oxaliplatin-based regimen after two desensitization protocols while one patient had a non-immediate HSR and one other had a discordant intradermal reaction. However one of three patients failed in re-treatment because of wrong infusion rate $(2 \mathrm{~h}$ instead of $6 \mathrm{~h}$ ). Conclusion: Oxaliplatin-desensitization procedures are feasible in oncology-day hospital. The success rate in our cohort is not explained by the hospitalization modalities but by patient selection for desensitization (according to initial grade or diagnostic of HSR). Multicentre studies are warranted in order to confirm our results and to standardize desensitization protocols in oncology-day hospital. Disclosure of Interest: None Declared.

PT033: A systematic review of clinicians' views and experiences of direct-acting oral anticoagulants

D. Generalova ${ }^{1, *}$, S. Cunningham ${ }^{1}$, S. Leslie $^{2}$, G. Rushworth ${ }^{3}$, L. McIver ${ }^{4}$, D. Stewart ${ }^{1}$

${ }^{1}$ School of Pharmacy and Life Sciences, Robert Gordon University, Aberdeen, ${ }^{2}$ Department of Medicine, Raigmore Hospital, ${ }^{3}$ 3Highland Pharmacy Education and Research Centre, Centre for Health Science, Inverness, ${ }^{4}$ Pharmacy, Healthcare Improvement Scotland, Glasgow, United Kingdom

Background and objective: Recent years have seen marked changes in the availability of oral anticoagulants. The introduction of dabigatran in 2008 was followed by other direct-acting oral anticoagulants (DOACs), rivaroxaban, apixaban and edoxaban. A plethora of systematic reviews have provided evidence of efficacy, effectiveness and safety in the management of non-valvular atrial fibrillation (AF). To date, no systematic reviews focusing on clinicians' perspectives have been reported. Given current prescribing levels and the expected trend towards increasing use, there is a need to for pooled data on their perspectives. The objective was to critically appraise, synthesise and present the available evidence of clinicians' views and experiences of DOACs for the management of non-valvular AF. 
Setting and method: A systematic review protocol was developed according to PRISMA-P standards (Preferred Reporting Items for Systematic review and Meta-Analysis Protocols) and registered with PROSPERO (International Prospective Register of Systematic Reviews). Search databases were: Cumulative Index to Nursing \& Allied Health Literature, International Pharmaceutical Abstracts, Medline and PsycARTICLES. All studies were assessed for quality and data extracted by two independent reviewers.

A narrative approach to data synthesis was undertaken due to heterogeneity of study outcome measures.

Main outcome measures: Synthesised findings of clinicians' views and experiences of prescribing and use of DOACs.

Results: Following exclusion of duplicates, 777 titles, 394 abstracts and 196 studies were screened. Ten studies were included in the review, nine quantitative (cross-sectional surveys) and one qualitative (semi-structured interviews). Studies were conducted exclusively in Europe and the United States. In those reporting clinician preference, DOACs were first choice over warfarin in naïve patients, based on perceptions of evidence of effectiveness equivalent or superior to warfarin and superior safety. Other advantageous factors were in those with an unstable INR and likely to miss appointments. There were concerns relating to management of over-anticoagulation and experiences of observed bleeding rates.

Conclusion: There is a limited evidence base of clinicians' perspectives of DOACs, necessitating further research, particularly given the trajectory of increased use worldwide. The limited evidence indicates DOACs as first choice over warfarin in naïve patients, those with an unstable INR and likely to miss appointments. There is a need for evidence based guidelines to support management of overanticoagulation.

Disclosure of Interest: None Declared.

\section{PH005: Health and economic impact of the discontinuation of lenalidomide treatment in patients with myelodysplastic syndrome associated with $\operatorname{del}(5 q)$}

\section{Alarcón-Payer ${ }^{1, *}$, M. Salazar Bravo ${ }^{1}$, A. Jiménez Morales ${ }^{1}$, R. García Fumero ${ }^{1}$, M. Jurado Chacón ${ }^{2}$}

${ }^{1}$ Servicio de Farmacia, ${ }^{2}$ Servicio de Hematología, Hospital Universitario Virgen de Las Nieves, Granada, Spain

Background and objective: According to OMS-2016, myelodysplastic syndrome (MDS) associated with del(5q) is manifested by a transfusion-dependent progressive bone marrow failure, with Lenalidomide acting as the intended drug to treat this syndrome. The aim of this study is to evaluate the clinical and economic impact associated to the discontinuation of the Lenalidomide treatment due to side effects or intolerance.

Design: Three-year prospective observational study on 69 cases of MDS, 17 of them with del(5q). Treatment discontinuation was studied in those candidates with side effects or intolerance. The variables considered in this study were: beginning of treatment, Lenalidomide mean dose, ending of treatment and beginning of discontinuation, side effects, time after discontinuation, evaluation of the drug withdrawal response according to IWG-MDS (Chenson et al. 2006), and cost savings.

Results: 69 MDS cases were analyzed (58\% male and $42 \%$ female). 17 cases were detected as MDS associated to $\operatorname{del}(5 q)(24.6 \%)$ and $17.6 \%$ of them showed positive TP53 mutation and were treated with hypomethylating agents instead of Lenalidomide. $64.7 \%$ of the cases were treated with Lenalidomide, the treatment were discontinued in $54.5 \%$ of them due to side effects and the dose reduced in $18 \%$ due to intolerance. The reported side effects were: Grade 4 neutropenia, rhabdomyolysis, erythematous reactions and haemolytic crisis. $100 \%$ of patients in which Lenalidomide was discontinued due to side effects, maintained complete haematological and cytogenetic response, reaching a mean monitoring time of 12 months since the withdrawal of Lenalidomide. The cost saving associated to the discontinuation of Lenalidomide $10 \mathrm{mg}$ was 48,000 euros per patient per year.

Conclusion: Discontinuation of Lenalidomide, due to side effects or intolerance, is assocaited with a clinical benefit in those patients who maintain a complete haematological response after interruption of the treatment. It also represents an economic saving of 48,000 euros per year per patient discontinued.

Disclosure of Interest: None Declared.

PH006: Professionals' perspectives about the tuberculosis control programme in Recife, Brazil: a contribution to evaluation

S. S. Bezerra ${ }^{1}$, M. P. Guerreiro ${ }^{2,3, *}$, M. P. G. Athayde ${ }^{4}$, J. J. F. Gomes ${ }^{5}$, J. L. S. Sobrinho ${ }^{6}$

${ }^{1}$ Post Graduation Program in Therapeutic Innovation, Federal University of Pernambuco, Recife, Pernambuco, Brazil, ${ }^{2}$ Unidade de Investigação \& Desenvolvimento em Enfermagem, Escola Superior de Enfermagem de Lisboa, Lisboa, ${ }^{3}$ Centro de Investigação Interdisciplinar, Instituto Superior de Ciências da Saúde Egas Moniz, Monte de Caparica, Portugal, ${ }^{4}$ Pneumology, Otávio de Freitas Hospital, Recife, Pernambuco, Brazil, ${ }^{5}$ Faculdade de Ciências, Universidade de Lisboa, Lisboa, Portugal, ${ }^{6}$ Pharmaceutical Sciences, Federal University of Pernambuco, Recife, Pernambuco, Brazil

Background and objective: In Brazil tuberculosis (TB) management involves multiple professionals working in the public health system across different levels of care. Pharmacists' responsibilities include the supply of anti-TB drugs and providing information to patients. This study aims to describe professionals' perspectives about the tuberculosis control programme (PCT) in Recife.

Setting and method: Five health units in Recife: two basic health units, one polyclinic, a university hospital of medium complexity and a hospital of high complexity. The study took place between 07/2014 and $02 / 2015$. Physicians, nurses, pharmacists, community agents and health managers working in the PCT were purposively sampled. Data were obtained by means of a questionnaire with 12 closed questions adapted from the literature, grouped into four categories (Coverage and Reception; Diagnosis, Clinical and Laboratory Assistance; Treatment and Pharmaceutical Assistance and Comprehensiveness of care). Answers were gathered on a five-point scale and converted into percentage scores before statistical analysis ( $\mathrm{R}$ software). Ethical approval was granted.

Main outcome measures: Total score per professional. Mean score per category.

Results: Respondents were mostly women $(87.5 \%, 21 / 24)$; the mean age was 40 years (minimum 30, maximum 55). Nurses and physicians comprised more than half of the respondents $(33 \%, 8 / 24$ and $25 \%$, $6 / 24$ respectively); followed by pharmacists $(n=5)$, managers $(\mathrm{n}=3)$ and community health agents $(\mathrm{n}=2)$. Overall, scores were below $60 \%$ (median $51.55 \%$, third quartile $64.6 \%$ ), evidencing an unfavorable perspective about the programme. Differences in the median scores by professional group did not reach statistically significance (Kruskal-Wallis test, $p=0.40$ ) Professionals working in the high complexity hospital presented a significantly less favourable opinion about the programme (Wilcoxson test; $p=0.025$ ). Professionals had a significantly worse opinion about the category "Diagnosis, Clinical and Laboratory Assistance" (Wilcoxson test; $p<0.01$ ), which includes items pertaining to registration and examination of contacts, case finding, smear results, sputum culture and sensitivity test when indicated, as well as rapid HIV test.

Conclusion: Professionals' perspectives on the PCT highlighted potential opportunities for improvement in the surveyed sites, in 
particular in an area with implications for the control of TB transmission. The lack of significant differences in the opinion of the five professional groups may be due to the absence of differences or reflect an insufficient sample size. Future studies evaluating the PCT should maintain a multidisciplinary approach, whilst seeking a larger, potentially more generalisable sample.

Disclosure of Interest: None Declared.

PH007: Safety culture in a large acute Irish teaching hospital: the Safety Attitudes Questionnaire

L. Gleeson ${ }^{1, *}$, L. Tobin ${ }^{1}$, E. Crowley ${ }^{1}$, A. Delaney ${ }^{2}$, D. O'Mahony ${ }^{3}$, S. Byrne

${ }^{1}$ Pharmaceutical Care Research Group, School of Pharmacy, University College Cork, ${ }^{2}$ Medication Safety, Cork University Hospital, ${ }^{3}$ Department of Medicine, University College Cork, Cork, Ireland

Background and objective: The Safety Attitudes Questionnaire (SAQ) is a validated instrument used in the measurement of safety culture. The aim of this study was to use the SAQ to carry out the first hospital-wide assessment of safety culture in Ireland.

Setting and method: Ethical approval was obtained from the local ethics committee. Surveys were distributed (electronically and by hand) to all staff in an Irish teaching hospital between December 2017 and January 2018. The attitudes of all hospital staff and individual professions towards six domains of safety culture were assessed over 32 Likert-scaled items. Results were analysed using SPSS. Thematic analysis was performed on an additional comments section of the survey to identify what staff regarded as important patient safety issues in the hospital.

Main outcome measures: Attitudes of hospital staff towards six domains of the SAQ.

Results: A total of 748 staff members completed and returned a copy of the SAQ. The majority of staff had positive attitudes towards teamwork climate $(75.6 \%)$, safety climate $(67.7 \%)$, job satisfaction $(67.52 \%)$ and stress recognition $(77.83 \%)$, while the percentages of staff with positive attitudes towards perceptions of management $(31.46 \%)$ and working conditions $(43.2 \%)$ were low. Nurses had a significantly more positive perception of teamwork climate than other professions (median 83.33, $p<0.005$ ), while doctors had significantly higher perceptions of stress recognition (median 87.5, $p<0.005)$. Thematic analysis of staff recommendations identified staffing levels, communication, resources, and training and education opportunities as areas that could be targeted to improve patient safety. Conclusion: The safety culture of an Irish teaching hospital was successfully assessed using the SAQ. This survey has given valuable insight into attitudes of hospital staff towards patient safety, and highlighted issues, such as working conditions and perceptions of management, which may inform further work on improving patient outcomes. Future work will include expansion of the survey to include other sites in Ireland, and development of an intervention to improve medication safety.

Disclosure of Interest: None Declared.

PH008: Medication errors associated with customized gentamicin dosing

E. Hagesaether ${ }^{1, *}$, H. Chairi ${ }^{2}$, M. Bayar ${ }^{2}$, A. G. Granas ${ }^{2}$

${ }^{1}$ OsloMet - Oslo Metropolitan University, ${ }^{2}$ University of Oslo, Oslo, Norway

Background and objective: Gentamicin is a broad spectrum antibiotic used for example against sepsis in children in a hospital setting.
Dosing is a balancing act between achieving the desired effect and avoiding renal and otic toxicity. Therefore, personalized dosing taking for example body weight and kidney function into account is required, in combination with therapeutic drug monitoring. The objective of this study was to assess medication errors associated with customized gentamicin dosing. More specifically to answer the following questions: what kind of errors occur most frequently, what age group are mostly affected and what are the causes and consequences. Setting and method: Medication errors reported from hospitals/ secondary care to a nation-wide error reporting system in 2016 in Norway. The method used to categorize errors was a modified version of the WHO Conceptual Framework for the International Classification for Patient Safety adjusted by the Norwegian Health Authorities. Main outcome measures: Analysis of written descriptions of errors and classification of medication errors involving gentamicin.

Results: In total, 23 out of 1881 reported medication errors involved gentamicin. Of these, 10 involved children between 0 and 4 years. 18 of the errors (78\%) occurred during the administration process and the remaining 5 errors occurred during the prescribing process. For 11 cases, failing communication/cooperation between health care workers was identified as a cause of error. In 7 cases procedures put in place were not followed and in 3 cases a lack of competence was reported. No major patient injuries were reported, but in 3 cases subsequently careful monitoring of the affected patients was required. The most common errors occurring during administration were omitted dose $(n=5)$ and wrong dosage/speed/strength $(n=4)$, and the most common error during prescription was wrong dosage $(\mathrm{n}=3)$.

Conclusion: The most frequent error involving gentamicin was omission during medicine administration, mainly caused by personnel failing to communicate with each other. Medication errors associated with customized gentamicin dosing disproportionally affected young children ( $0-4$ years). The consequences for the patients were mild, but required extra monitoring. Our detailed analysis of nationally reported medication errors is the first step towards producing learning-material to improve patient safety.

Disclosure of Interest: None Declared.

PH009: Pharmacy students' knowledge and attitudes toward organ transplantation and donation

S. Altay ${ }^{1}$, H. Köse ${ }^{1, *}$, O. Ozkan', M. Sancar', B. Okuyan ${ }^{1}$

${ }^{1}$ Clinical Pharmacy, Marmara University Faculty of Pharmacy, Istanbul, Turkey

Background and objective: The aim of this study is to evaluate the knowledge and attitude of pharmacy students toward organ transplantation and donation.

Setting and method: This study was conducted among pharmacy students from all grades at a pharmacy faculty in Istanbul, Turkey.

Main outcome measures: The Turkish form of 32-item questionnaire, previously developed by Fontana et al. (1), was administered to assess pharmacy students' their factual knowledge, personal views, and perceptions toward organ donation and transplantation. The cultural adaptation and validation was performed before using Turkish version of this questionnaire. The higher score represented better knowledge and attitude toward organ transplantation and donation.

Results: Of the 475 questionnaires delivered, 453 were returned by the pharmacy students, giving a response rate of $95.37 \%$. The mean of age was calculated as $22.08 \pm 1.84$ in all completed questionnaire $(n=453)$. Of them, $75.5 \%$ were female. Cronbach alpha coefficient was 0.951 . Of them, $93.80 \%$ declared their support for organ donation. However, only $6.20 \%$ registered to an organ donation list. Fourth and fifth degree students had statistically higher scores of the knowledge and attitude toward organ donation when compared with 
lower degree students (first, second and third) $(p<0.05)$. In such a manner, statistically higher scores of the knowledge and attitude were detected in students who were organ donors $(n=34)$, those who know somebody waiting for a transplant $(n=19)$, those who know somebody donated his or her organ $(\mathrm{s})(\mathrm{n}=77)$ and those who know somebody received an organ $(\mathrm{n}=57)$ when compared with nonothers $(p<0.05)$.

Conclusion: The students with personal knowledge of a situation involving organ transplantations or donating process have high level of awareness and knowledge about the donation and transplantation. A practical course can be developed in pharmacy training for bringing together students and the patients with the organ transplantation process, especially in the early years of education. In this way, pharmacists, as one of the most accessible health care professionals of public, could be more conscious to increase donation rates after graduation.

\section{Reference}

1. Fontana F, Massari M, Giovannini L, Alfano G, Cappeli G. Knowledge and Attitudes Toward Organ Donation in Health Care Undergraduate Students in Italy. Transplantation Proceedings. Transplantation Proceedings, 2017; 49, 1982-1987.

Disclosure of Interest: None Declared.

PH010: A prospective study to evaluate drug use among pregnant women

\section{R. M. Umar ${ }^{1, *}$, E. K. Koçberber ${ }^{2}$, R. Besiroglu ${ }^{3}$, A. K. Sahin ${ }^{3}$ \\ ${ }^{1}$ Clinical Pharmacy, Istanbul Medipol University, ${ }^{2}$ Clinical Pharmacy, Istanbul University, ${ }^{3}$ Istanbul Medipol University, Istanbul, Turkey}

Background and objective: Drug use is common among pregnant women. During pregnancy, drugs may be needed for the treatment of acute health conditions, pregnancy complications, fetus related conditions and/or already present chronic diseases. Drugs are prescribed for $80 \%$ of pregnant women and the majority of women tend to use these prescribed medications. The use of over-the-counter medications and herbal supplements are also common during pregnancy.

Setting and method: This prospective study was conducted in 300 randomly selected women who visited the department of gyneacology at a university-affiliated hospital. Patient information was collected by a pharmacist via the patient profile registration form. Medications and herbal supplements were classified according to the FDA pregnancy risk classification.

Main outcome measures: The purpose of the study is to evaluate the frequency of drug use among pregnant women and the category of drugs used.

Results: The results showed that $97.67 \%$ of these women were using at least 1 medication. A total of 553 different drugs were evaluated. $61.85 \%$ of these drugs were classified as category A, $6.9 \%$ as B, $25.39 \%$ as $\mathrm{C}, 0.78 \%$ as D and category of $5.03 \%$ was unknown. It was also determined that $73 \%$ of these women use herbal products. There is a negative correlation between education level and drug use before the confirmation of pregnancy $(p<0.05)$. In other words, patients with high education levels may have avoided medications considering their possibility of being pregnant. But the use of herbal supplements was higher in these patients. There is a significant correlation $(p<0.05)$ between the use of drugs prior to the confirmation of pregnancy and number of previous abortions and a negative correlation $(p<0.05)$ with gravidity and parity.

Conclusion: As the potential teratogenic effect of many drugs is yet to be determined, the use of drugs during pregnancy should be minimized to only when necessary. Pregnant women and females within the birth bearing age should be adequately guided in medication use. The most appropriate medication should be chosen for those with chronic diseases.

Disclosure of Interest: None Declared.

RD002: Pharmacogenetics of oral antidiabetic treatment in type 2 diabetes

D. Blanquez Martinez ${ }^{1}$, M. Hayon Ponce ${ }^{2}$, I. Casas Hidalgo', X. Diaz Villamarin ${ }^{1}$, R. Alvarez Sanchez ${ }^{1, *}$, P. Nieto Gomez ${ }^{1}$, C. Davila Fajardo ${ }^{1}$

${ }^{1}$ Farmacia Hospitalaria, ${ }^{2}$ Endocrinologia y Nutricion, Hospital Universitario Campus De La Salud, Granada, Spain

Background and objective: Pharmacogenetics is an emerging field that guide treatment selection based on genetic information. The objective in this review is to summarize the current knowledge about genetic markers of antidiabetic drugs, and to discuss the application of pharmacogenetics in clinical practice.

Setting and method: A search of electronic databases (PubMed, EMBASE, and Cochrane Database) was performed to identify original studies of the effect of diabetes medications in type 2 diabetes (T2D) by genetic variation from 2007 to 2018.

Main outcome measures: Specific gene polymorphisms of antidiabetic drugs.

Results: We included 52 articles comprised of 10,536 subjects. The included articles evaluated metformin, sulfonylureas, meglitinides, thiazolidinediones, DPP-4 inhibitors, GLP-1 agonist and SGLT-2 inhibitors. Medication-gene interactions included: (1) Metformin: SLC22A1, SLC22A2, SLC47A1; (2) Sulfonylureas: KCNJ11, ABCC8, CYP2C9, TCF7L2; (3) Meglitinides: SLCO1B1, CYP2C8, CYP3A4, TCF7L2, SLC30A8, KCNJ11, KCNQ1; (4) Thiazolidendiones: AQP2, SLC12A1, PPAR $\gamma$, PAX4, ADIPOQ, CYP2C8; (5) DPP-4 inhibitors: DPP4; (6) SGLT-2 inhibitors: SLC5A2.

Conclusion: Significant pharmacogenetic evidence has demonstrated an association between specific gene polymorphisms and interindividual variability in glucose-lowering drugs and side effects. Several variants related to drug-metabolizing enzymes, drug-transporters, drug target, and diabetes risk genes have been linked to interindividual differences in the glucose-lowering agents outcomes. Identification of drug-genotype interactions in pharmacogenetic studies of the antidiabetic treatment might have clinical implications in the selection of more specific personalized therapy in T2D.

Disclosure of Interest: None Declared.

RD003: Developing social media guidelines for undergraduate pharmacy students

\section{A. Brown ${ }^{1, *}$, K. MacLure ${ }^{1}$}

${ }^{1}$ Robert Gordon University, Aberdeen, United Kingdom

Background and objective: Literature suggests that undergraduate (UG) healthcare students should have social media (SoMe) guidelines that support their context as future professionals. Currently students are expected to use either profession specific guidelines or institutional guidelines to support their use of SoMe. Guidelines may address the blurred lines between professional and personal use of SoMe which have led to confusion over potential fitness to practise (FtP) consequences from inappropriate online behaviours. The aim of this study was to explore UG Master of pharmacy (MPharm) student views of eprofessionalism, appropriate online behaviours and the content and delivery of SoMe guidelines.

Design: An online survey was sent to MPharm students in 22 institutions across UK and Ireland. Questions explored the definition of 
eprofessionalism, characteristics of appropriate/inappropriate online behaviour, consequences of inappropriate behaviours, content and delivery of SoMe guidelines. The study was given ethical approval from the School of Pharmacy and Life Sciences Ethical Review Committee.

Results: A total of 341 students from all MPharm stages responded representing all areas of the UK and Ireland. Although respondents agreed with the definition of eprofessionalism, there was a perceived need for the definition to be more 'usable', with a need to update terminology. Whilst there was agreement on what behaviours were 'appropriate', there was little consensus on what the consequences of 'inappropriate' behaviours should be. Students do not readily use SoMe guidelines but suggest they should be accessible with a variety of methods used to engage students effectively.

Conclusion: There remains a lack of awareness of the potential consequences for inappropriate online behaviours. Guidelines should include examples of appropriate and inappropriate behaviours with discussion of the potential FtP consequences. Further work is underway to develop guidelines based on the recommendations from this study.

Disclosure of Interest: None Declared.

\section{RD004: Exploring the role of pharmacy teams in Scottish GP} practice: an interim analysis

\section{Stewart ${ }^{1, *}$, M. Bennie ${ }^{2}$, K. MacLure ${ }^{1}$, R. Newham ${ }^{2}$, K. Gibson Smith ${ }^{1}$, S. Cunningham ${ }^{1}$, R. Bruce ${ }^{2}$, S. Fry ${ }^{3}$, J. MacKerrow ${ }^{3}$}

${ }^{1}$ Robert Gordon University, Aberdeen, ${ }^{2}$ University of Strathclyde, Glasgow, ${ }^{3}$ Information Services Division, Edinburgh, United Kingdom

Background and objective: Scottish Government policy has highlighted the potential contribution of pharmacy teams, comprising pharmacists and pharmacy technicians, in GP practices across Scotland. The aim of this study is to explore the role of pharmacy teams in Scottish GP practice. The study is in progress and accordingly, the following should be regarded as interim data.

Setting and method: An online questionnaire was developed in consultation with pharmacists, pharmacy technicians and experts in pharmacy education and policy. The questionnaire underwent both Think Aloud $(n=6)$ and pilot testing $(n=22)$ with pharmacists and pharmacy technicians. An electronic link was sent on behalf of the research team by contacts at each Scottish Health Board to all pharmacists and pharmacy technicians working in GP Practices across Scotland. Board contacts were asked to send two reminder emails at fortnightly intervals.

Main outcome measures: Pharmacist and pharmacy technician activites in GP practice.

Results: Interim response rates for pharmacists were $79.3 \%$ (371/468) and for pharmacy technicians, $86.4 \%$ (95/110). The majority of pharmacists and pharmacy technicians were aged between 30 and 49 years $(\mathrm{n}=132,35.6 \% ; \mathrm{n}=66,69.5 \%$ respectively $)$ and had been qualified for 20 or more years $(n=149,40.2 \% ; n=33,34.7 \%$ respectively). Pharmacists reported working in up to 29 GP Practices $($ Mode $=2)$ and pharmacy technicians across 1-54 (Mode $=5$ ). Pharmacists were undertaking medication/polypharmacy reviews face-to-face $(\mathrm{n}=213,57.4 \%)$, notes only $(\mathrm{n}=145,39.1 \%)$ and via telephone/video $(\mathrm{n}=92,24.8 \%)$. Over $60 \%$ were currently undertaking prescribing efficiency work $(\mathrm{n}=276,74.4 \%)$ and interpreting prescribing data $(\mathrm{n}=266,71.7 \%)$. The largest numbers of pharmacists were currently undertaking polypharmacy $(\mathrm{n}=191$, $51.5 \%)$, respiratory $(\mathrm{n}=74,20.0 \%)$ and pain $(\mathrm{n}=69,18.6 \%)$ clinics. Pharmacy technicians were undertaking medication/polypharmacy reviews face-to-face $(\mathrm{n}=8,8.4 \%)$, notes only $(\mathrm{n}=33,34.7 \%)$ and via telephone/video $(\mathrm{n}=4,4.2 \%)$. In addition, over $60 \%$ were currently undertaking prescribing efficiency work $(\mathrm{n}=74,77.9 \%)$ and interpreting prescribing data $(n=66,69.5 \%)$.

Conclusion: The findings illustrate the depth of the pharmacy workforce who are employed in GP practices across Scotland, comprising both pharmacists and pharmacy technicians. They further highlight the diverse roles of pharmacists and pharmacy technicians employed in GP practice. These interim results will be used to guide further research, involving database analysis and focussed case studies, on pharmacy teams in GP practice across Scotland.

Disclosure of Interest: None Declared.

RD005: Exploring perspectives around non-medical prescribing: an umbrella review

T. Jebara ${ }^{1, *}$, D. Stewart ${ }^{1}$, S. Cunningham ${ }^{1}$, K. MacLure ${ }^{1}$, A. Awaisu ${ }^{2}$, A. Palli Valapila ${ }^{3}$

${ }^{1}$ School of Pharmacy and Life Sciences, Robert Gordon University, Aberdeen, United Kingdom, ${ }^{2}$ College of Pharmacy, Qatar University, ${ }^{3}$ Women's Hospital, Hamad Medical Corporation, Doha, Qatar

Background and objective: Many countries have implemented nonmedical prescribing (NMP) and many others are scoping prescribing practices with a view to developing NMP. Thus, a comprehensive description of global published review articles around NMP has the potential to aid further development.

This umbrella review aims to collate and summarise all the published reviews on NMP to provide insight on its future perspectives.

Setting and method: MEDLINE, CINAHL, ScienceDirect ${ }^{\circledR}$, International Pharmaceutical Abstracts, Cochrane Library, CRD Prospero, Joanna Briggs Institute database and Google Scholar were searched for any review article discussing any aspect of NMP. To assess the quality of systematic reviews and protocols, CASP, PRISMA and PRISMA-P tools were applied.

Main outcome measures: Any aspects of non-medical prescribing including but not limited to:

- models and definitions of NMP discussed

- impact

- perceptions and satisfaction

Results: The review identified seven systematic reviews, four protocols and 13 other types of reviews around influences on prescribing decision-making, processes of prescribing, and facilitators and barriers to implementation of NMP. Decision-making was reported as complex with many, and often conflicting, influences. Reviews that explored patient outcomes reported NMP as equivalent to or better than physician prescribing. Facilitators of NMP included perceived improved patient care and professional autonomy, while barriers included lack of defined roles and resource pressures.

Conclusion: While evidence of benefit and safety is essential to inform practice, for NMP to be implemented and sustained on a large scale, there needs to be clear commitment at the highest policy level. Challenges to NMP could be met by considering the theoretical basis for implementation, and robust and rigorous evaluation.

Disclosure of Interest: None Declared. 